Tesis de Doctorado:

\title{
RADIACIÓN GAMMA EN \\ BINARIAS CON ACRECIÓN
}

Lic. Mariana D. Orellana

Director: Dr. Gustavo E. Romero

Grupo de Astrofísica Relativista y Radioastronomía

Facultad de Ciencias Astronómicas y Geofísicas Universidad Nacional de La Plata

Paseo del Bosque S/N, (1900) La Plata, Argentina

Diciembre de 2007 



\section{Agradecimientos}

Quiero agradecer a mi director, Gustavo Romero por haberme guiado durante estos años en mis primeras investigaciones. Por su esfuerzo en general y por las oportunidades que me ha brindado. Por su apoyo y paciencia.

Agradezco al Dr. J.M. Paredes, por la oportunidad de conocer su grupo de trabajo en Barcelona. Gracias Valenti y Pol por mostrarme su ciudad, y por la colaboración profesional.

I want to thank for useful and interesting lessons shared by Professor Atsuo Okazaki.

Gracias a Leonardo Pellizza por enseñarme qué es un Monte Carlo, y haber atendido a todas mis consultas.

Agradezco al personal y autoridades del Instituto Argentino de Radiastronomía y de la Facultad de Cs. Astronómicas y Geofísicas por su colaboración e interés en la presente tesis. Agradezco al Consejo Nacional de Investigaciones Científicas y Técnicas por haberme otorgado la beca que me permitió dedicarme en forma exclusiva a esta investigación. También agradezco al CONICET, la fundación ANTORCHAS y la ANPCyT por los subsidios que hicieron posible tener los elementos para trabajar.

Aprecio la compañía, incluso a la distancia, de las chicas del GARRA. En especial, gracias Ileana por el aliento en esta última etapa.

Agradezco al grupo de estrellas Be (Modelos de Estrellas Peculiares) de la FCAGLP por los amenos seminarios a los que me han invitado.

Saliendo de este ámbito científico, los agradecimientos personales los debo a quienes han estado sosteniéndome, aguantando y alentando durante esta etapa. A Fer, por todo. A mamá. Y a mis queridas amigas que han bancado mis nervios e inestabilidades.

Finalmente, quisiera dedicar esta tesis a la memoria de Maria Elena Viola, en eterno agradecimiento por su comprensión. 


\section{Resumen}

Entre los problemas más importantes de la astrofísica se destaca la falta de una clara identificación para las fuentes detectadas a muy altas energías $(>100 \mathrm{MeV})$ por telescopios satelitales. En la actualidad, detecciones a energías más altas aún $(>200 \mathrm{GeV})$ están siendo obtenidas por telescopios Cherenkov. En combinación con estudios multifrecuencia, estas observaciones plantean numerosos desafíos teóricos.

Entre las posibles contrapartes de las fuentes gamma galácticas, los sistemas binarios con acreción brindan excelentes perspectivas, a las que se suma la reciente detección en el rango $\mathrm{TeV}$ de 4 binarias de rayos $\mathrm{X}$. En la presente investigación doctoral nos hemos focalizado en la emisión gamma originada en binarias de gran masa con acreción, debido a la interacción de protones relativistas con el medio local. Dicho proceso genera radiación gamma a través del decaimiento de los piones neutros que se crean en las colisiones $p p$. Específicamente, hemos estudiado el caso de un objeto compacto de bajo campo magnético, capaz de efectuar eyecciones de masa confinadas en chorros de partículas relativistas (microcuásares). En este contexto, hemos explorado también la emisión leptónica secundaria. Dentro de los jets se generan pares relativistas al decaer piones cargados, que son otro subproducto de las colisiones $p p$. Se resolvió en forma consistente la evolución de esta población de pares, teniendo en cuenta las pérdidas de energía radiativas y adiabática, y se calculó su emisión sincrotrón y Compton inversa.

La controvertida fuente LS I +61303 ha sido estudiada introduciendo un modelo de microcuásar hadrónico para su emisión $\gamma$. A tal fin se modificó el modelo de Romero et al. (2003) para incluir la geometría del disco circunestelar que aporta los protones blanco y la variabilidad en la tasa de acreción de masa, que a su vez, impone una limitación energética a la potencia llevada por los jets. Hemos colaborado en la implementación de simulaciones dinámicas (SPH) tridimensionales para la transferencia de masa en LS I +61 303. Se compararon los valores obtenidos para la tasa de acreción con estimaciones analíticas, donde se asume que la acreción es esférica y ocurre al estar el objeto compacto inmerso dentro del disco circunestelar de la primaria. Además, éstas y otras simulaciones nos permitieron contrastar la factibilidad de un escenario de acreción/eyección contra uno de colisión de vientos para explicar el comportamiento de esta fuente. 
Por otro lado, estudiamos el caso donde el objeto acretante es una estrella de neutrones altamente magnetizada, analizando en particular la emisión de muy altas energías durante una explosión gigante de rayos X. Durante esta explosión, un disco de acreción transitorio se forma en torno a la estrella de neutrones. En la magnetósfera del pulsar, el mecanismo de Cheng y Ruderman puede producir una corriente de protones relativistas que se dirigen hacia el disco, donde impactan produciendo rayos gamma e iniciando cascadas hadrónicas y electromagnéticas. Estudiamos por separado ambos procesamientos.

En los casos contemplados, donde la binaria contiene una estrella de tipo espectral joven, la propagación de los rayos $\gamma$ muy energéticos generados en el volumen del sistema binario puede inducir el desarrollo de cascadas electromagnéticas dominadas por el mecanismo de dispersión Compton inversa. Hemos desarrollado un código para la simulación Monte Carlo de cascadas unidimensionales desarrolladas en el campo radiativo térmico y anisotrópico de una estrella de gran masa. Se exponen algunos de los resultados obtenidos, poniendo en relieve la importancia de este problema para la correcta estimación de un espectro detectable a energías mayores que algunos $\mathrm{GeV}$. 


\section{Índice general}

\begin{tabular}{ll}
\hline Agradecimientos & I
\end{tabular}

Resumen III

Siglas utilizadas $\quad$ IX

1. Introducción 1

2. Contexto observacional 3

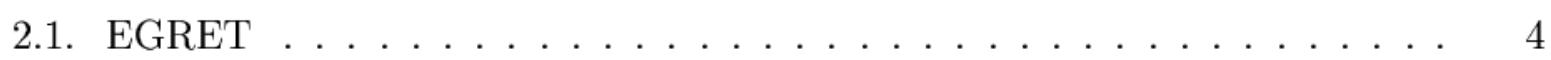

2.2. Detecciones en la era Cherenkov . . . . . . . . . . . . . . 6

3. Binarias de rayos 11

3.1. Vientos en estrellas de gran masa . . . . . . . . . . . . . . . 13

3.2. Pulsares . . . . . . . . . . . . . . . . . . . . . . 15

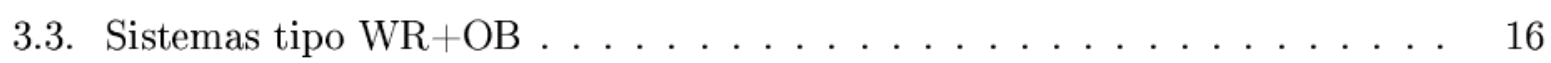

3.4. Sistemas con colisión de viento pulsar-estrella . . . . . . . . . . . . 17

4. Acreción y evección de materia en binarias de gran masa 21

4.1. Discos de acreción en sistemas binarios . . . . . . . . . . . . . . . 22

4.1 .1$. La corona . . . . . . . . . . . . . . . . . . 25

4.12 Transferencia de masa . . . . . . . . . . . . . . . . . 27

4.2. Jets v microcuásares . . . . . . . . . . . . . . . . . 27

4.3. Estados espectrales: hacia un modelo unificado . . . . . . . . . . . 30

4.4. Microcuásares en la Galaxia . . . . . . . . . . . . . . . . . . . . . 34 
$\begin{array}{ll}\text { 5. Modelos para la emisión } \gamma \text { de microcuásares } & 37\end{array}$

5.1. Modelos leptónicos . . . . . . . . . . . . . . . . . . . . . . . . . . . . . 39

5.2. Modelos hadrónicos . . . . . . . . . . . . . . . . . . . . . . . 40

5.2.1. La distribución de protones relativistas . . . . . . . . . . . . . 41

5.2.2. Cálculo de la emisión gamma hadrónica . . . . . . . . . . 43

6. Propagación de los ravos 45

6.1. Cascadas electromagnéticas . . . . . . . . . . . . . . . . 46

6.2. El código desarrollado v algunas aplicaciones . . . . . . . . . . . . . 49

7. Un modelo hadrónico para la emisión $\gamma$ del microcuásar LS I +61 $303 \quad 55$

7.1. Características de la fuente . . . . . . . . . . . . . . . . . 55

7.2. El modelo . . . . . . . . . . . . . . . . . . . . . . . . . . . . 59

7.3. Controversias . . . . . . . . . . . . . . . . . 63

7.3.1. Simulaciones de colisión de vientos . . . . . . . . . . . 64

7.3.2. Simulaciones de transferencia de masa . . . . . . . . . . . 67

8. Emisión de leptones secundarios 73

8.1. Generación de leptones secundarios . . . . . . . . . . . . . . . . . . 74

8.1.1. Evolución de la población de leptones . . . . . . . . . . . . . . 75

8.2. Aplicación en un sistema similar a Cyg X-1 . . . . . . . . . . . . 76

9. Microcuásares con jets inclinados $\quad 81$

9.1. Difusión hacia las regiones internas del viento estelar . . . . . . . . . . 84

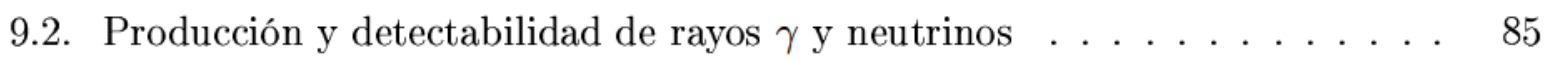

9.2.1. Caso de interacción directa con la superficie de la estrella . . . . . . 88

$\begin{array}{ll}\text { 10.Emisión gamma en pulsares acretantes } & 91\end{array}$

10.1. Aceleración de partículas en la magnetósfera . . . . . . . . . . . . . . . . . 93

10.1.1. Producción de ravos gamma y procesamiento dentro del disco de acreción 95

10.1.2. Procesamiento en la fotósfera . . . . . . . . . . . . . 99

\begin{tabular}{ll}
\hline 1. Conclusiones y perspectivas & 101
\end{tabular}

\begin{tabular}{ll}
\hline Bibliografia & 105
\end{tabular} 


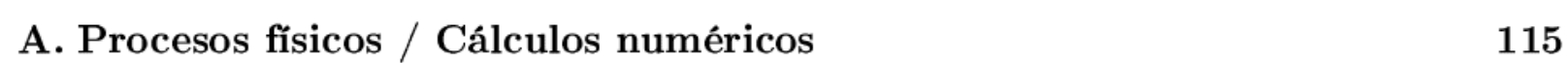

A.1. Aceleración de partículas en frentes de choque . . . . . . . . . . . . . . 115

A.2. Interacciones hadrónicas . . . . . . . . . . . . . . . . . . 117

A.3. Cálculo detallado de la absorción en el campo radiativo estelar . . . . . . . 119

A.3.1. Dispersiones IC en un campo anisotrópico . . . . . . . . . . . 123

A.4. Nociones sobre simulaciones Monte Carlo . . . . . . . . . . . . . . . . 124

A.5. Las ecuaciones que describen una cascadas electromagnética . . . . . . 126

$\begin{array}{ll}\text { Lista de publicaciones } & 129\end{array}$ 


\section{Siglas utilizadas}

En la mayoría de los casos se han mantenido las siglas en inglés, ya que son de uso más amplio que en castellano.

$\begin{array}{ll}\text { ADAF } & \text { Advection Dominated Accretion Flow } \\ \text { AGILE } & \text { Astro-rivelatore Gamma Immagini Leggero } \\ \text { AGN } & \text { Active Galactic Nuclei } \\ \text { BATSE } & \text { Burst and Transient Source Experiment } \\ \text { CM } & \text { (sistema de referencia del) Centro de Momentos } \\ \text { CGRO } & \text { Compton Gamma-Ray Observatory } \\ \text { COMPTEL } & \text { Compton Telescope } \\ \text { EAS } & \text { Extensive Air Shower } \\ \text { EGRET } & \text { Energetic Gamma-Ray Experiment Telescope } \\ \text { GLAST } & \text { Gamma-ray Large Area Space Telescope } \\ \text { GRB } & \text { Gamma-Ray Burst } \\ \text { HESS } & \text { High Energy Stereoscopic System } \\ \text { HMXB } & \text { High Mass X-ray Binary } \\ \text { IR } & \text { Infra-Rojo } \\ \text { LMXB } & \text { Low Mass X-ray Binary } \\ \text { MAGIC } & \text { Major Atmospheric Gamma Imaging Cherenkov } \\ \text { MERLIN } & \text { Multi-Element Radio-Linked Interferometer Network } \\ \text { MILAGRO } & \text { Multiple Institution Los Alamos Gamma Ray Observatory } \\ \text { MQ } & \text { Microquasar } \\ \text { OSSE } & \text { Oriented Scintillation Spectrometer Experiment } \\ \text { PWN } & \text { Pulsar Wind Nebula } \\ \text { QPO } & \text { Quasi-Periodic Oscillations } \\ \text { RXTE } & \text { Rossi X-ray Timing Explorer } \\ \text { SPH } & \text { Smoothed Particle Hydrodynamics } \\ \text { UA } & \text { Unidad Astronómica } \\ \text { UV } & \text { Ultra-Violeta } \\ \text { VERITAS } & \text { Very Energetic Radiation Imaging Telescope Array System } \\ \text { VLA } & \text { Very Large Array }\end{array}$


VLBA Very Long Baseline Array

VLBI Very Long Baseline Interferometry

WR Wolf Rayet 


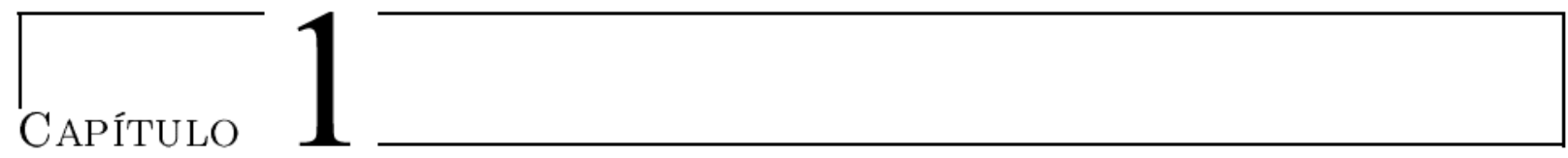

\section{Introducción}

Durante las dos última décadas la astronomía gamma ha evolucionado, pasando de ser una rama casi exótica de la astronomía, a establecerse como un campo de investigación por sí misma, permitiéndonos vislumbrar algunos de los escenarios más interesantes y a la vez complejos del Universo. De hecho, entre los problemas más importantes de la astrofísica se destaca la falta de una clara identificación para las fuentes detectadas a muy altas energías $(>100 \mathrm{MeV})$ por telescopios satelitales. Este es el caso para la mayoría de las fuentes observadas por el instrumento EGRET del satélite Compton que no han podido correlacionarse en forma clara con fuentes detectadas a más bajas energías.

Bajo esta motivación, el objetivo principal del plan propuesto para esta tesis fue el obtener predicciones teóricas en diferentes escenarios astrofísicos, relevantes a la producción de radiación gamma, que puedan contrastarse con resultados observacionales. El actual impulso instrumental para la observación a altas energías (de fotones y neutrinos) ha hecho particularmente oportuno el propósito de esta investigación.

Las poblaciones de fuentes de radiación gamma actualmente confirmadas incluyen, por nombrar algunas, a núcleos galácticos activos, remanentes de supernovas, agujeros negros acretantes, pulsares, eventos explosivos de rayos gamma, etc. Todos estos ejemplos incluyen situaciones físicas muy diferentes de las que podemos estudiar en laboratorios, dándose diversos fenómenos violentos que ocurren en regímenes relativistas, a veces en presencia de campos magnéticos o radiativos muy intensos, y donde puede ocurrir la aceleración de partículas hasta muy altas energías.

En cuanto a las fuentes gamma en el disco galáctico, a las cuales se presta particular atención en esta tesis, muchas de ellas son bastante débiles y variables, con escalas temporales de variación que sólo pueden originarse en regiones de dimensiones menores que la estelar, como los entornos de objetos compactos, ya sean estrellas de neutrones o agujeros negros. Es por esto que, entre las posibles contrapartes para las fuentes gamma no identificadas, los modelos de sistemas binarios con acreción brindan excelentes perspectivas. En el transcurso de esta tesis nos hemos focalizado en la emisión gamma originada por la 
interacción de protones relativistas con el medio local. Específicamente, hemos estudiado el caso donde el objeto acretante es una estrella de neutrones altamente magnetizada y por otra parte el caso de un objeto compacto de bajo campo magnético, capaz de efectuar eyecciones de masa confinadas en chorros de partículas relativistas. Estos últimos sistemas se denominan microcuásares.

La estrella donante del material acretado en los casos contemplados es de tipo espectral temprano, por lo que su campo radiativo ofrece un blanco adecuado para la absorción de fotones gamma, posiblemente iniciando cascadas electromagnéticas. La propagación de los rayos gamma desde el lugar de su emisión plantea así un problema complejo, que debemos abordar al intentar modelar un espectro detectable. A tal fin, hemos desarrollado un código que permite la simulación Monte Carlo de cascadas electromagnéticas unidimensionales.

A lo largo de esta tesis se da primero un breve capítulo introductorio al contexto observacional, a continuación se introduce la fenomenología de los escenarios estudiados y se comentan los avances realizados en la investigación, encontrándose a partir del capítulo 7 la mayoría de los aportes originales. 


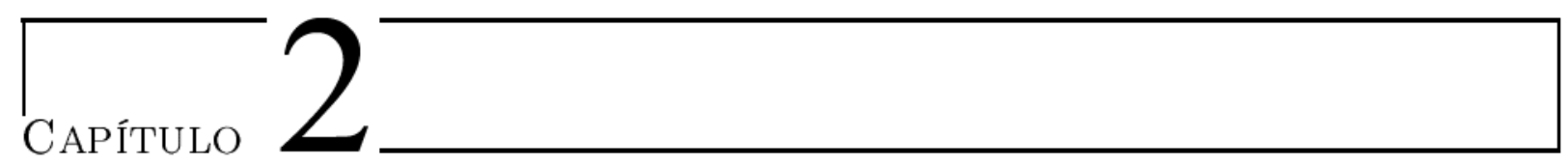

\section{Contexto observacional}

Los fotones con energías por encima de los $500 \mathrm{keV}$ son llamados en general rayos gamma. Su estudio, la astronomía gamma, se enfoca sobre los procesos no térmicos en el Universo, siendo el único rango del espectro electromagnético libre de contribuciones producidas por plasmas calientes por lo que la radiación emitida por encima de $1 \mathrm{MeV}$ se debe casi enteramente a interacciones de partículas relativistas.

Excepto a energías realmente muy grandes $(\geq 10 \mathrm{GeV})$, la recepción de estos fotones desde tierra se ve imposibilitada por la absorción de la atmósfera, y debe hacerse utilizando experimentos satelitales. Tales experimentos han sido lanzados desde la década de los '60, propiciados por el desarrollo instrumental de la época. Sin embargo, la incipiente astronomía gamma tardó décadas en arrojar resultados significativos. Esto se debió a dificultades técnicas específicas y al formidable problema de separar las contribuciones producidas en el detector por fuentes legítimas de rayos $\gamma$ de las interacciones de los rayos cósmicos (partículas cargadas 1 y neutrones relativistas) con el material del propio receptor, que generan en forma local nuevos fotones gamma. De hecho, la medición de rayos $\gamma$ de origen cósmico enfrenta desafíos enormes al ser el ruido de fondo mucho mayor que la señal a detectar en esta banda.

Dado que los rayos gamma interaccionan mediante diferentes procesos a diferentes energías, las técnicas de detección varían con la frecuencia de los fotones a detectar. Este es uno de los motivos por los cuales se subdivide al espectro gamma en varias bandas: bajas energías a $E<30 \mathrm{MeV}$, altas energías entre $30 \mathrm{MeV}$ y $30 \mathrm{GeV}$, muy altas energías entre $30 \mathrm{GeV}$ y $30 \mathrm{TeV}$, ultra altas energías entre $30 \mathrm{TeV}$ y $30 \mathrm{PeV}\left(1 \mathrm{PeV}=10^{15} \mathrm{eV}\right)$ y energías extremas para $E>30 \mathrm{PeV}$. A lo largo de esta tesis nos referimos mayormente a la banda de altas y muy altas energías cuando se hable de fotones $\gamma$.

\footnotetext{
${ }^{1}$ Mayormente núcleos atómicos, siendo un $87 \% \mathrm{H}, 12 \%$ He y $1 \%$ de C, N, O, Fe. Las energías de estas partículas siguen un espectro que puede ser descripto como ley de potencias, siendo sus únicos rasgos salientes un ligero ablandamiento a algunos $\mathrm{PeV}$ (la rodilla) y endurecimiento a los $10 \mathrm{EeV}$ (el tobillo).
} 
La tecnología de receptores con cámaras de destello\$2 resultó apropiada para superar la barrera del ruido de fondo que afecta a la detección de rayos $\gamma$ de altas energías. Los principales instrumentos que utilizaron esta tecnología han sido el SAS-2 (1972), el COS-B de la ESA (1975 - 1982) y EGRET (1991 - 2000). El enorme avance que aportó EGRET a la astronomía gamma es brevemente comentado en la próxima sección. Los telescopios actuales que operan por creación de pares utilizan detectores de silicio en vez de cámaras de destellos. Las nuevas misiones que volarán incorporan esta tecnología: GLAST (el GAMMAray Large Area Space Telescope, que operará en el rango $20 \mathrm{MeV}-300 \mathrm{GeV}$, y con una sensibilidad que superará en dos órdenes de magnitud la de EGRET) y el satélite italiano AGILE, ya lanzado y operando, entre otras.

Fotones de muy alta energía inician en la atmósfera cascadas electromagnéticas que permiten una detección indirecta, a través de observaciones de la luz Cherenkov que generan las partículas secundarias de la cascada. Durante el intervalo entre el cese de operaciones de EGRET en el 2000 y el recientemente concretado lanzamiento de nuevas misiones satelitales (AGILE en 2007), las únicas observaciones de rayos $\gamma$, mayormente a energías $>50 \mathrm{GeV}$, han sido hechas desde tierra mediante esta técnica. Los principales detectores Cherenkov que se encuentran operando son HESS y MAGIC. La sección 2.2 describe esta técnica de observación, y sus principales resultados a la fecha.

\subsection{EGRET}

El telescopio EGRET (Energetic Gamma Ray Experiment Telescope) fue uno de los cuatro instrumentos que formaban parte del observatorio satelital Compton. El CGRO (CGRO: COMPTON GAmma RAY ObSERVATORY) monitoreó el cielo en rayos gamma durante 9 años, desde 1991 y fue una de las misiones más exitosas de la NASA. Además de EGRET, también lo conformaban BATSE, un instrumento optimizado para detectar variaciones de flujo muy rápidas (fuentes transitorias y GRB) en el rango de 30 $\mathrm{keV}$ a $1.9 \mathrm{MeV}$; OSSE que operaba en el rango de 0.05 a $10 \mathrm{MeV}$; y COMPTEL que trabajaba en el rango de 1 a $30 \mathrm{MeV}$. EGRET, era el instrumento para las energías más altas: $20 \mathrm{MeV}$ a $30 \mathrm{GeV}$, con un campo angular grande y resolución angular dependiente de la energía de los fotones; siendo su mejor resolución de medio grado a $5 \mathrm{GeV}$. A lo largo de la mayoría de su rango de detección la resolución en energías era del $20-25 \%$.

Del total de las fuentes descubiertas por EGRET, 271 detecciones a energías superiores a $100 \mathrm{MeV}$ fueron incorporadas en el tercer catálogo que fue la lista oficial final del telescopio

\footnotetext{
${ }^{2}$ En inglés spark chamber, también se suele llamar cámara de chispas. Podemos decir en forma simplificada que consiste de una cámara sellada y llena de un gas a presión, de modo que el pasaje de un electrón o positrón deja una estela de ionización. Los pares son formados en la interacción del fotón $\gamma$ con el material del aparato que ofrece su superficie como área colectora. Dentro de la cámara hay una serie de placas paralelas conectadas de modo tal de detectar las chispas que se producen en el gas, permitiendo determinar la trayectoria de la partícula. La principal limitación de la cámara de chispas es que el contenido de gas se agota, y a medida que esto sucede el rendimiento del instrumento se va deteriorando.
} 
(Hartman et al. 2001). El criterio adoptado para definir las fuentes "3EG" fue el de una detección 3 con significancia mayor que $5 \sigma$ por sobre el ruido de fondo, para las fuentes del plano galáctico y de $4 \sigma$ a latitudes mayores que $|b|=5^{\circ}$. Entre las fuentes detectadas se incluyeron unos 70 núcleos galácticos activos (AGNs), mayormente del tipo BL-Lacertae o blazares, 1 radio galaxia (Centaurus A), la nube mayor de Magallanes y 6 radio pulsares. Las demás, unas 170 fuentes que son casi dos terceras partes del total permanecen sin identificación, esto es, no han podido encontrarse contrapartes claras en otras longitudes de onda. La baja resolución angular de EGRET es una de las principales causas para que esto sea así.

La distribución en el cielo de las fuentes no identificadas indica que varias de ellas pertenecen a nuestra galaxia (ver Figura 2.1). Algunas se han conocido por más de 20 años, permaneciendo como un misterio desde los mapeos realizados por COS-B (Swanenburg et al. 1981). Se han propuesto diversos estudios estadísticos que permiten clasificar a estas fuentes en grupos con características comunes. Gehrels et al. (2000) han tenido en cuenta los índices espectrales de las fuentes no identificadas estables (no variables) hallando una división entre fuentes brillantes con espectros más duros a bajas latitudes galácticas y fuentes más débiles y con espectros blandos que se ubican en latitudes intermedias $\left(5^{\circ} \leq|b| \leq 30^{\circ}\right)$. Los análisis de correlación de Romero et al. (1999) indican que, espacialmente, las fuentes gamma 3EG no identificadas del plano galáctico siguen distribuciones similares a las de los remanentes de supernovas y asociaciones de estrellas $\mathrm{OB}$, lo cual reforzó resultados previos en que se usaron muestras menores. Entre las nuevas posibilidades discutidas sobre la naturaleza de estas fuentes podemos mencionar agujeros negros de Kerr-Newmann (Punsly et at 2000), binarias Be/X (Romero et al 2001), y microcuásares (Paredes et al. 2000, Kaufman-Bernadó et al. 2002, Romero et al. 2003). Se han propuesto también estrellas masivas con vientos fuertes (Benaglia et. al 2001, Benaglia \& Romero 2003), y remanentes de supernova interactuando con nubes atómicas o moleculares (Combi et al. 2001; Butt et al. 2001, 2002; Torres et al. 2003). Finalmente, se ha anunciado una nueva población de fuentes a grandes distancias del plano galáctico, probablemente del halo (Grenier 2001). Contrapartes atractivas para estas fuentes muy viejas son pulsares de milisegundos (ej. Torres, Butt \& Camilo 2001) y microcuásares de baja masa (Kaufman-Bernadó 2004 y referencias).

Ciertamente, mucho trabajo es aún necesario para poder confirmar o rechazar muchas de las posibles explicaciones, especialmente las más especulativas. La búsqueda de una identificación para estos emisores de rayos gamma se ha convertido en uno de los problemas más importantes de la astrofísica de altas energías. Más aún, el origen de la radiación gamma para algunas de las fuentes cuya contraparte está bien establecida se encuentra en debate, como es el caso de LS I +61303 , o es pobremente entendida. En el caso de fuentes detectadas por HESS (veremos a continuación), en algunos casos se dá una coincidencia posicional, pero los datos multifrecuencia no parecen apuntar hacia un escenario consistente.

\footnotetext{
${ }^{3}$ Durante la compilación del catálogo se consideró este criterio en diferentes bandas de energía, generando algunas discrepancias que han motivado recientes re-analisis, Reimer \& Funk (2007).
} 


\subsection{Detecciones en la era Cherenkov}

Los rayos cósmicos llegan a la tierra en forma isotrópica a una tasa $\mathrm{m}^{-2}$. Un flujo muy pequeño comparado a este es el que forman rayos $\gamma$ y neutrinos que al viajar en forma directa desde sus fuentes permiten desarrollar una astronomía en el sentido estricto. Los rayos $\gamma$ y rayos cósmicos más energéticos constituyen las partículas primarias de lluvias atmósfericas (EAS: ExTEnsive AIr Showers), o cascadas electromagnéticas que se desarrollan en la atmósfera. Los detalles de los procesos físicos de estas lluvias fueron originalmente descriptos por Rossi y Greisen (1941) y pueden hallarse versiones actualizadas, por ejemplo, en Gaisser (1990). Actualmente se recurre a simulaciones numéricas para estudiar el problema altamente complejo del desarrollo/reconstrucción de lluvias atmósfericas.

La primera interacción de una lluvia se produce a unos $30 \mathrm{~km}$ de altura. En esta interacción se generan partículas secundarias, que de nuevo interactuan con las moléculas de la atmósfera para generar más partículas y/o fotones gamma (radiación Bremsstrahlung). Mediante la repetición de estos procesos se desarrolla la cascada. Al principio el número de partículas en la lluvia crece rapidamente, pero al distribuirse la energía entre más subproductos, se llega en algun momento a estar por debajo del umbral de creación de nuevas partículas, y las que quedan se enfrían en forma radiativa o colisional, de modo que la cascada se detiene. Al tratarse de procesos relativistas, las partículas que resultan de la cascada estan colimadas en la dirección de la inicial, mientras que su emisión Cherenkov abarca al llegar a la tierra una zona de unos $100 \mathrm{~m}$ de radio (light pool).

\footnotetext{
${ }^{4} \mathrm{El}$ flujo de rayos cósmicos es dependiente de la energía de las partículas, que siguen el espectro que ya hemos comentado. Así las protones más energéticos $\left(\sim 10^{20} \mathrm{eV}\right)$ llegan a la tierra a una tasa menor a uno por $\mathrm{km}^{2}$ por siglo.
}
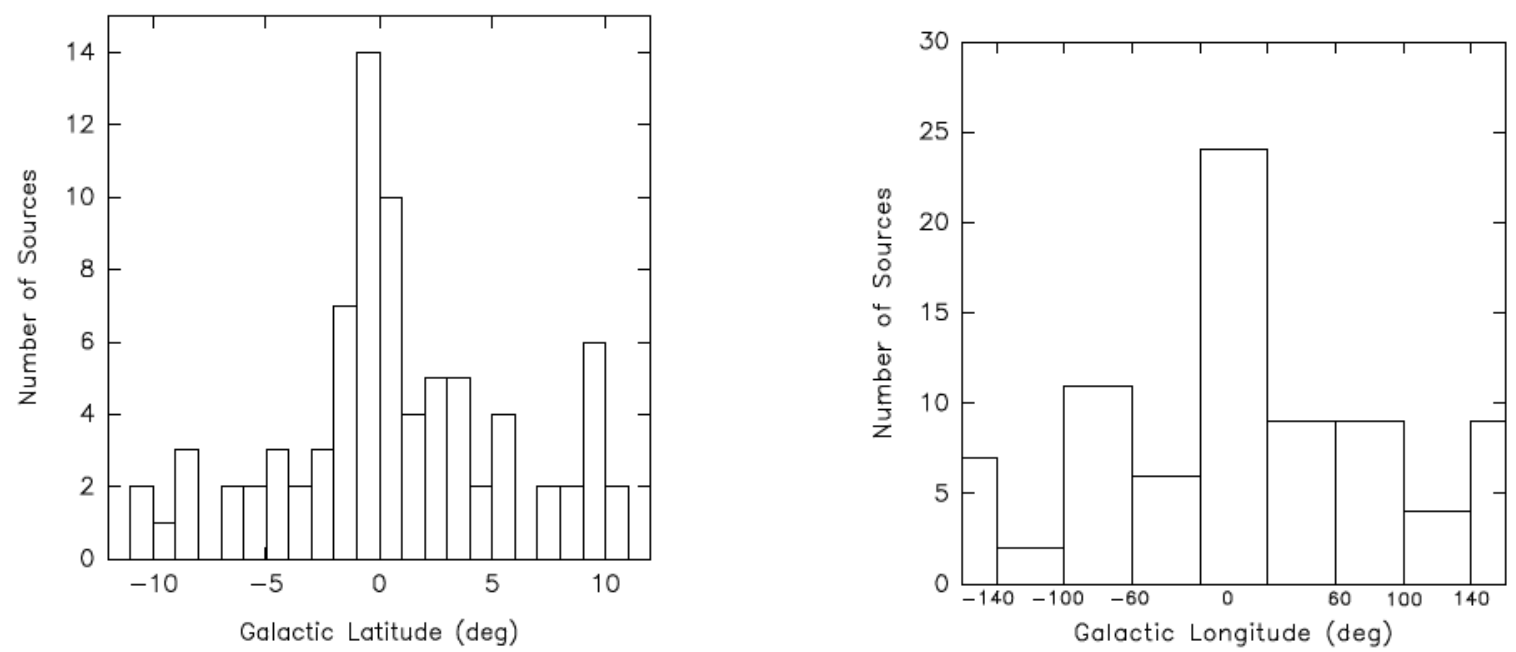

Figura 2.1: Distribución en latitudes (izquierda) y longitudes galácticas (derecha) de 81 fuentes no identificadas con $|b|<10^{\circ}$, del Tercer Catálogo EGRET (Romero et al. 1999). 


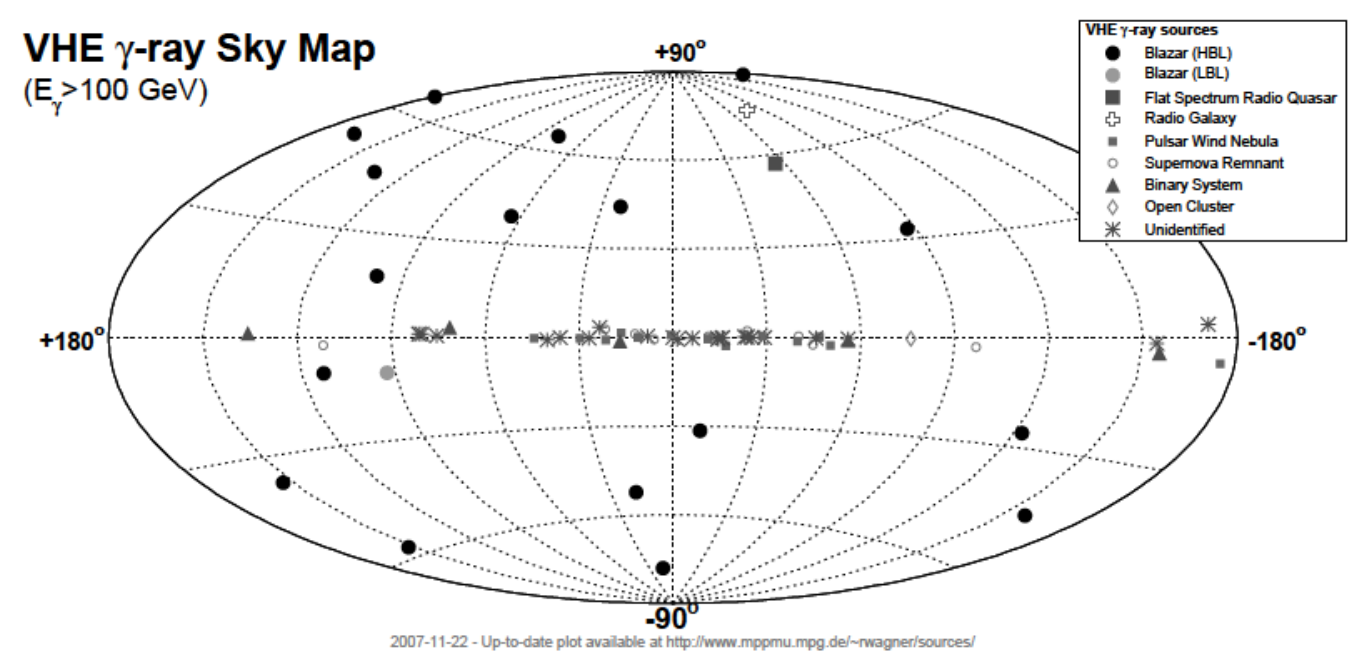

Figura 2.2: El cielo en rayos $\gamma$ : ubicación de las fuentes detectadas a energías $>100 \mathrm{GeV}$, en coordenadas galácticas. Versiones más actualizadas de este gráfico de R. Wagner pueden encontrarse en http://www.mppmu.mpg.de/ rwagner/sources/

La técnica de detección por mapeo (Cherenkov) de estas cascadas se basa en la recepción de la luz que emiten ${ }^{5}$ las partículas secundarias de la cascada al moverse más rápido que la velocidad de la luz en la atmósfera. Dada la dependencia del índice de refracción atmosférico con la frecuencia, esta luz cae en el rango óptico y es recolectada por una serie de fototubos en el plano focal de un telescopio reflector. En general, existen diferencias que permiten distinguir las lluvias iniciadas por rayos cósmicos de las originadas por fotones $\gamma$. Esto es cierto también para las partículas de la lluvia y de hecho, existen experimentos (ubicados a una altura conveniente) como MILAGRO, que detectan fuentes $\gamma$ basandose en la detección de partículas que recolectan en forma permanente (sus resultados se resumen por ej. en Abdo et al., 2007). Por otro lado, el flash Cherenkov de una cascada tiene una duración de apenas unos nanosegundos, haciendo necesaria una respuesta muy veloz del detector. Además, excelentes condiciones atmósfericas y ausencia de luna son necesarias para obtener datos confiables, lo que impone un bajo rendimiento del tiempo de observación.

Un arreglo de varios telescopios ayuda a una mejor reconstrucción de la trayectoria de la cascada, y así la posición de una fuente para la cual se recojan unos cientos de fotones puede llegar a establecerse con una precisión de algunos minutos de arco (tan buena o mejor que la de observaciones satelitales). Un esquema de la lluvia, y un arreglo detector puede verse en la Figura 2.3

La primera detección confiable mediante la técnica Cherenkov fue reportada en 1989 , y fue obtenida con el telescopio reflector de $10 \mathrm{~m}$ de diámetro del Observatorio Whipple6.

\footnotetext{
${ }^{5}$ En rigor, la radiación Cherenkov es el resultado de la reorientación de los dipolos inducidos en las moléculas del aire por una partícula cargada que se mueve suficientemente rápido.

${ }^{6} \mathrm{La}$ fuente en cuestión era la nebulosa del Cangrejo (Crab), que se ha convertido en la calibradora de referencia de la astronomía en el rango $\gamma$.
} 


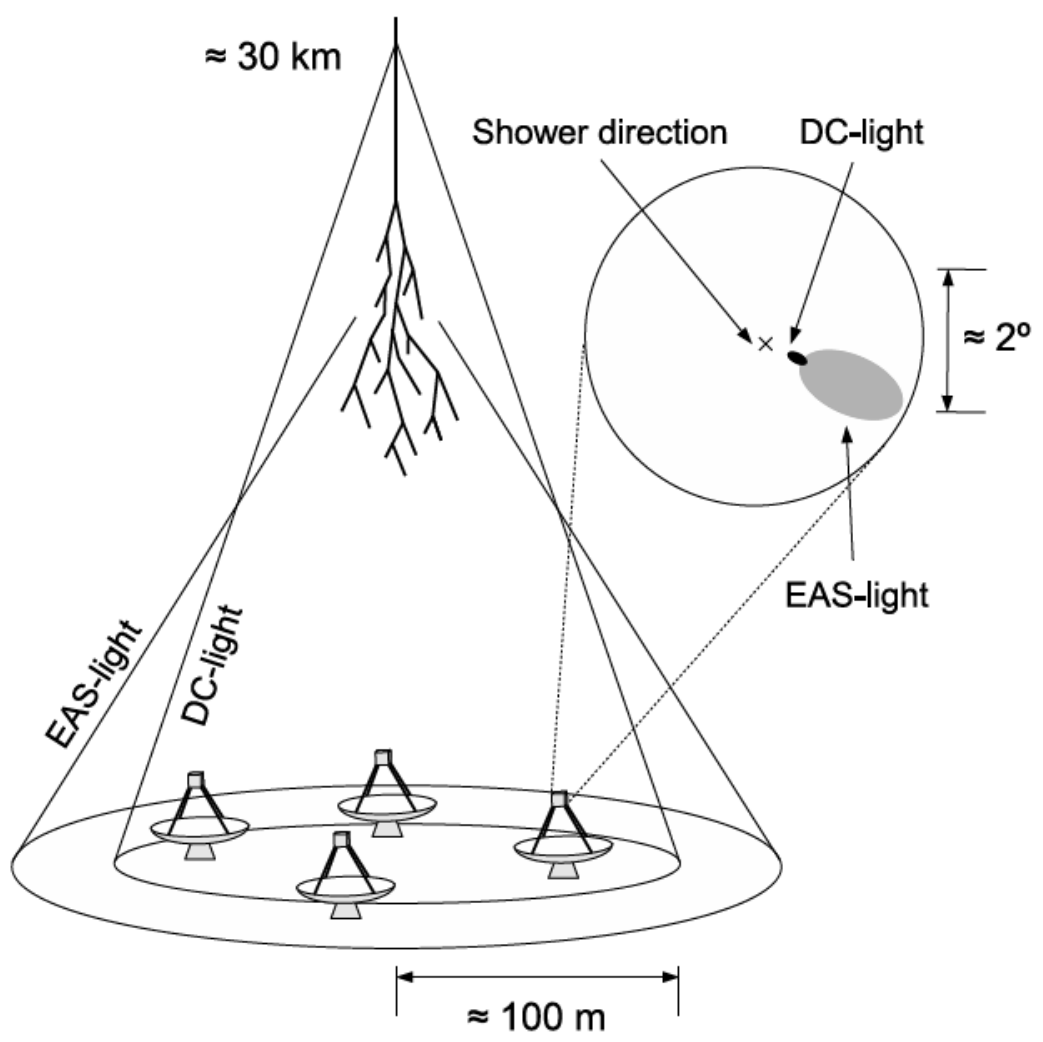

Figura 2.3: Esquema de una lluvia atmósferica y un arreglo Cherenkov para su detección (Aharonian et al. 2007).

HESS, el High Energy Stereoscopic System ha completado en 2004 el primer relevamiento del plano galáctico, con una sensibilidad de un pequeño porcentaje de Crab a energías mayores que $100 \mathrm{GeV}$. El telescopio MAGIC (MAJOR ATMOSPheric GaMma Imaging Cherenkov) que es el de mayor diámetro $(17 \mathrm{~m})$ operando mediante esta técnica ha aportado también resultados muy interesantes. Para detalles sobre MAGIC ver Domingo-Santamaría (2006). Los artículos de revisión que listan las fuentes TeV confirmadas quedan desactualizados con velocidad creciente al ser este un campo tan activo. En la Tabla 2.1 pueden verse las fuentes galácticas y extragalácticas reportadas antes de Julio de 2006, habiendo referencias para cada una en Cui (2006). Vale la pena comentar que la emisión difusa en el rango TeV tiene un especial interés cosmológico al permitir el estudio indirecto de la radiación infrarroja de fondo con la que interactúa 7 . Debe agregarse a la lista de microcuásares la detección de Cygnus X-1, anunciada muy recientemente por el equipo de MAGIC (Albert et al. 2007).

Actualmente, gracias a colaboraciones internacionales, se planean y se están construyendo otros grandes arreglos Cherenkov que permiten preveer un incremento enorme en el

\footnotetext{
${ }^{7}$ Fotones con energía mayor que $E_{\gamma} \sim 1 \mathrm{TeV}$ son absorbidos por los infrarrojos para crear pares e iniciar cascadas electromagnéticas dominadas por el proceso Compton inverso, como las que se discutirán más adelante. En este caso, los caminos libres medios para la interacción $\gamma \gamma$ son del orden de algunos Mpc.
} 
número de fuentes $\mathrm{TeV}$ en los años próximo\$ 8 . Además, aumentando el área colectora del arreglo y la altura en que se ubica, se intentará bajar la energía mínima de los fotones $\gamma$ detectables desde tierra, de modo de reducir la banda no cubierta por observaciones que hay entre dicha energía y la energía máxima detectable por los observatorios satelitales.

${ }^{8}$ HESS II y MAGIC II son extensiones respectivas de los instrumentos existentes. VERITAS (USA) es un arreglo similar a HESS. CTA es un proyecto de gran envergadura que podría incluir hasta $\sim 100$ telescopios. 
Capítulo 2. Contexto observacional

Tabla 2.1: Fuentes TeV reportadas a Julio de 2006, por Cui (2006)

\begin{tabular}{|c|c|c|c|c|}
\hline Clase & Nombre & RA $(2000)$ & $\operatorname{Dec}(2000)$ & Notas \\
\hline Blazar & $\begin{array}{l}\text { 1ES 1101-232 } \\
\text { Mrk } 421 \\
\text { Mrk } 180 \\
\text { 1ES } 1218+304 \\
\text { H } 1426+428 \\
\text { PG } 1553+113 \\
\text { Mrk } 501 \\
\text { 1ES } 1959+650 \\
\text { PKS } 2005-489 \\
\text { PKS } 2155-304 \\
\text { 1ES } 2344+514 \\
\text { H } 2356-309\end{array}$ & $\begin{array}{lll}11 & 03 & 37.57 \\
11 & 04 & 27.31 \\
11 & 36 & 26.41 \\
12 & 21 & 21.94 \\
14 & 28 & 32.6 \\
15 & 55 & 43.04 \\
16 & 53 & 52.22 \\
19 & 59 & 59.85 \\
20 & 09 & 25.39 \\
21 & 58 & 52.07 \\
23 & 47 & 04.92 \\
23 & 59 & 07.8\end{array}$ & $\begin{array}{lll}-23 & 29 & 30.2 \\
+38 & 12 & 31.8 \\
+70 & 09 & 27.3 \\
+30 & 10 & 37.1 \\
+42 & 40 & 29 \\
+11 & 11 & 24.4 \\
+39 & 45 & 36.6 \\
+65 & 08 & 54.7 \\
-48 & 49 & 53.7 \\
-30 & 13 & 32.1 \\
+51 & 42 & 17.9 \\
-30 & 37 & 38\end{array}$ & \\
\hline FR I & M 87 & 123049.42 & +122328.0 & \\
\hline $\begin{array}{l}\text { Viento } \\
\text { de pulsar } \\
\text { (PWN) }\end{array}$ & $\begin{array}{l}\text { Crab Nebula } \\
\text { Vela X } \\
\text { G313.3+0.1 } \\
\text { K3/Kookaburra } \\
\text { MSH 15-52 } \\
\text { G18.0-0.7 }\end{array}$ & $\begin{array}{lll}05 & 34 & 31.97 \\
08 & 33 & 32 \\
14 & 18 & 04 \\
14 & 20 & 09 \\
15 & 14 & 07 \\
18 & 26 & 03.0\end{array}$ & $\begin{array}{l}+220052.1 \\
-454342 \\
-605831 \\
-604836 \\
-590927 \\
-134544\end{array}$ & $\begin{array}{l}\text { PSR B0532+21 } \\
\text { PSR B0833-45 } \\
\text { "Rabbit" (R2/Kookaburra) } \\
\text { PSR J1420-6048 } \\
\text { PSR B1509-58; composite } \\
\text { PSR J1826-1334 }\end{array}$ \\
\hline $\begin{array}{l}\text { Remanente de } \\
\text { Supernova } \\
\text { tipo } \\
\text { cáscara }\end{array}$ & $\begin{array}{l}\text { RX J0852.0-4622 } \\
\text { RX J1713.7-3946 } \\
\text { G0.9+0.1 } \\
\text { G12.82-0.02 } \\
\text { Cas A }\end{array}$ & $\begin{array}{lll}08 & 52 & 00 \\
17 & 13 & 00 \\
17 & 47 & 23.2 \\
18 & 13 & 36.6 \\
23 & 23 & 24\end{array}$ & $\begin{array}{lll}-46 & 20 & 00 \\
-39 & 45 & 00 \\
-28 & 09 & 06 \\
-17 & 5035 \\
+58 & 48 & 54\end{array}$ & $\begin{array}{l}\text { "Vela Junior" } \\
\text { G347.3-0.5 } \\
\text { composite }\end{array}$ \\
\hline Microcuásar & $\begin{array}{l}\text { LS } 5039 \\
\text { LS I }+\mathbf{6 1} \mathbf{3 0 3}^{\dagger}\end{array}$ & $\begin{array}{l}182615 \\
024031.67\end{array}$ & $\begin{array}{l}-145053.6 \\
+611345.6\end{array}$ & \\
\hline Binaria $\mathrm{Be} / \mathrm{X}$ & PSR B1259-63 & 130247.65 & -635008.7 & \\
\hline $\begin{array}{l}\text { Fuente } \\
\text { no identificada: } \\
\text { con contraparte } \\
\text { pausible }\end{array}$ & $\begin{array}{l}\text { HESS J1616-508 } \\
\text { HESS J1632-478 } \\
\text { HESS J1634-472 } \\
\text { HESS J1640-465 } \\
\text { HESS J1713-381 } \\
\text { HESS J1745-290 } \\
\text { HESS J1804-216 } \\
\text { HESS J1834-087 } \\
\text { HESS J1837-069 }\end{array}$ & $\begin{array}{lll}16 & 16 & 23.6 \\
16 & 32 & 08.6 \\
16 & 34 & 57.2 \\
16 & 40 & 44.2 \\
17 & 13 & 58.0 \\
17 & 45 & 41.3 \\
18 & 04 & 31.6 \\
18 & 34 & 46.5 \\
18 & 37 & 37.4\end{array}$ & $\begin{array}{lll}-50 & 53 & 57 \\
-47 & 49 & 24 \\
-47 & 16 & 02 \\
-46 & 31 & 44 \\
-38 & 11 & 43 \\
-29 & 00 & 22 \\
-21 & 42 & 03 \\
-08 & 45 & 52 \\
-06 & 56 & 42\end{array}$ & $\begin{array}{l}\text { PSR J1617-5055 } \\
\text { IGR J16320-4751 } \\
\text { G337.2+0.1/IGR J16358-4726 } \\
\text { G338.3-0.0/3EG J1639-4702 } \\
\text { G348.7+0.3 } \\
\text { G359.95-0.04/SgrA East/SgrA* } \\
\text { G8.7-0.1/PSR J1803-2137 } \\
\text { G23.3-0.3 } \\
\text { G25.5+0.0/AX J1838-0655 }\end{array}$ \\
\hline $\begin{array}{l}\text { Fuente } \\
\text { no identificada } \\
\text { sin } \\
\text { contraparte }\end{array}$ & $\begin{array}{l}\text { HESS J1303-631 } \\
\text { HESS J1614-518 } \\
\text { HESS J1702-420 } \\
\text { HESS J1708-410 } \\
\text { HESS J1745-303 } \\
\text { TeV J2032+4131 }\end{array}$ & $\begin{array}{llll}13 & 03 & 00.4 \\
16 & 14 & 19.0 \\
17 & 02 & 44.6 \\
17 & 08 & 14.3 \\
17 & 45 & 02.2 \\
20 & 31 & 57\end{array}$ & $\begin{array}{llll}-63 & 11 & 55 \\
-51 & 49 & 07 \\
-42 & 04 & 22 \\
-41 & 04 & 57 \\
-30 & 22 & 14 \\
+41 & 29 & 56.8\end{array}$ & -3011 \\
\hline $\begin{array}{l}\text { Emisión } \\
\text { difusa }\end{array}$ & $\begin{array}{l}\text { Galactic Ridge } \\
\text { Cygnus Region }\end{array}$ & - & - & \\
\hline
\end{tabular}

${ }^{\dagger}$ También una binaria Be. 


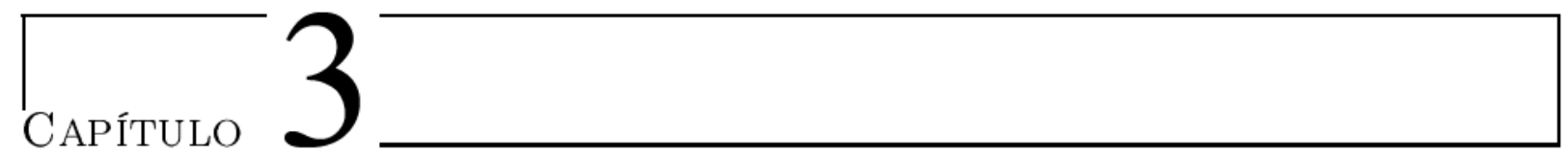

\section{Binarias de rayos $\gamma$}

Catalogadas desde un principio como potenciales fuentes gamma, los sistemas binarios con emisión de rayos X han sido blanco de diversas especulaciones teóricas. El interés por estos modelos se vió intensificado a partir de la confirmación observacional que brindaron los arreglos Cherenkov: algunas binarias de rayos $\mathrm{X}$ emiten, con intensidad variable, fotones con energía mayor que $100 \mathrm{GeV}$. A la fecha, se conocen 4 binarias de rayos gamma: LS 5039 y PSR B1259-63 detectadas por HESS (Aharonian et al. 2005a, Aharonian et al. 2005b respectivamente); LS I +61 303 y Cygnus X-1 detectadas por MAGIC (Albert et al. 2006, Albert et al. 2007 respectivamente).

Los modelos que describan en forma adecuada a las binarias de rayos $\gamma$ deben en última instancia explicar su emisión a lo largo de practicamente todo el espectro electromagnético, y muchas veces comportamientos variables en el tiempo que difieren según la banda de observación. A modo de ejemplo, la Figura 3.1 muestra la distribución espectral de energía observada para PSR B1259-63 y LS 5039. La emisión de LS I +61 303 será tratada por separado más adelante, ya que ha sido nuestro caso concreto de estudio.
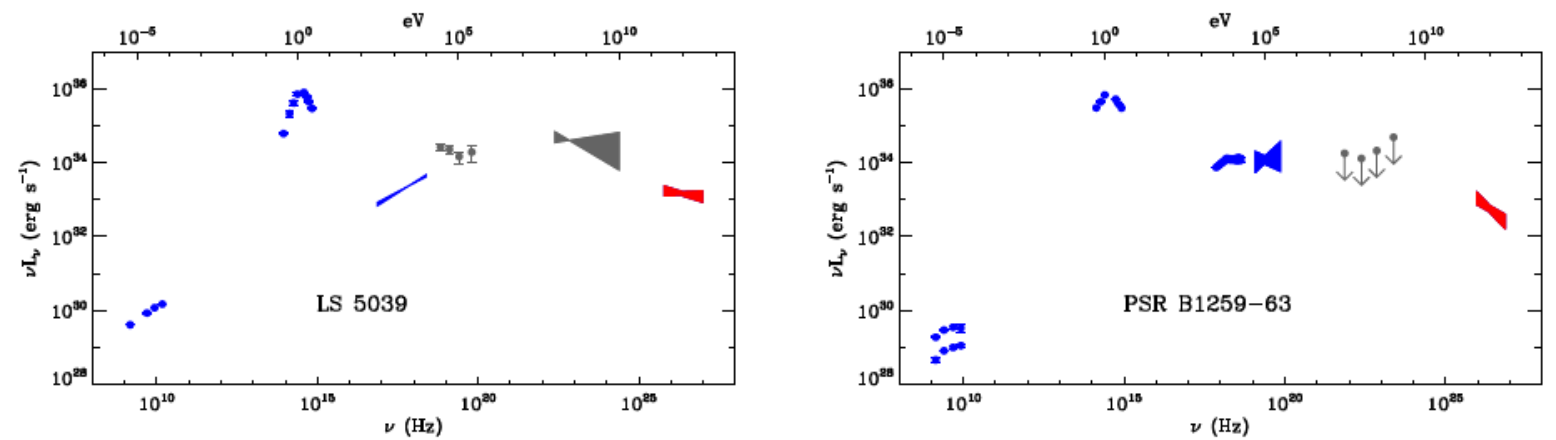

Figura 3.1: Distribución espectral de energía observada para LS 5039 y PSR B1259-63. Dubus(2006a). Los flujos se han transformado en luminosidades asumiendo una distancia de $2.5 \mathrm{y}$ $1.5 \mathrm{kpc}$, respectivamente. Se recopilan datos no simultáneos, con referencias en el artículo mencionado. 
Las fuentes mencionadas pertenecen al grupo de binarias X de gran masa (HMXB: High MASS X-RAY BINARY), esto es, contienen una estrella de gran masa (tipo espectral $\mathrm{O}$ - B) y un objeto compacto, ya sea una estrella de neutrones o un agujero negro. Con excepción de PSR B1259-63, se cree que en estas fuentes la emisión X está vinculada a la acreción de materia donada por la estrella normal sobre el objeto compacto. Esto también puede ocurrir en binarias X de baja masa (LMXB: LOW MASS X-RAY BINARY), marcando la diferencia entre ambas clases no sólo la masa de la estrella normal, sino también el mecanismo responsable de la transferencia de masa. En las HMXB interviene el viento producido por la estrella, que genera una tasa de pérdida $\dot{M}_{\text {wind }} \sim 10^{-8}-10^{-5} \mathrm{M}_{\odot}$ $\mathrm{yr}^{-1}$. Los mecanismos que actúan impulsando estos vientos, su configuración y emisión son altamente complejos y merecen su propia área de estudio (ver Sección 3.1). En las LMXBs la estrella normal ha evolucionado, saliendo de la secuencia principal para expandirse más allá de cierta superficie equipotencial que la contiene y se dice que la transferencia de masa ocurre por derrame del lóbulo de Roche.

Otra posibilidad para la emisión de rayos X (y tal vez $\gamma$ ) por una binaria es que se trate de un sistemas con colisión de vientos. Sistemas formados por dos estrellas muy tempranas brindan esta posibilidad, tratándose usualmente de sistemas tipo $\mathrm{WR}+\mathrm{OB}$. El frente de choque estacionario que se forma allí donde se equiparan las presiones de ambos vientos es el sitio donde se aceleran las partículas que luego emiten radiación $\gamma$. Por otro lado, la interacción del viento de una estrella de gran masa con el viento de un pulsar lleva a una situación con ciertas similitudes. Este escenario requiere que el pulsar sea lo bastante jóven como para tener una tasa de pérdida de energía rotacional importante $\left(\dot{E}_{\text {rot }} \sim 10^{36}\right.$ erg $\mathrm{s}^{-1}$ ). Un ejemplo de estos sistemas, con emisión $\gamma$ confirmada, es PSR B1259-63. Estudios cinemáticos de LS 5039 y LS I +61 303 muestran que han sido expulsados de "cunas" cercanas por explosiones de supernova que ocurrieron dentro del pasado millón de años (Mirabel, 2006). Por lo tanto, si hay un pulsar en estos sistemas, se trata de uno que en efecto es relativamente jóven.

Mayormente, la naturaleza de la estrella compacta en las binarias X no es conocida, pues mediante técnicas espectroscópicas 1 en el rango visual la masa suele estar acotada en un rango que permite ambas posibilidades: estrella de neutrones o agujero negro. La emisión en radio en forma de pulsos probablemente sea absorbida en forma eficiente por el material denso del viento estelar a menos que el pulsar se aleje lo suficiente de la estrella durante parte de su movimiento orbital2. Si esto ocurre y se detectan pulsaciones en radio $\mathrm{y} / \mathrm{o}$ rayos $\mathrm{X}$ estaremos en presencia de una estrella de neutrones, y puede resultar más probable el mecanismo de interacción de vientos entre pulsar y estrella 3 . Sobre las dos fuentes mencionadas permanece en debate (Dubus, 2006a) si es éste el escenario o más

\footnotetext{
${ }^{1}$ Se determina la función de masa, que provee una cota inferior de la masa del objeto compacto. Por lo general se desconoce la inclinación de la órbita, y se adopta un valor de masa para la estrella normal que tiene una gran incerteza, especialmente para estrellas de gran masa.

${ }^{2}$ Esto es lo que ocurre en el sistema de PSR B1259-63, que tiene una órbita muy excéntrica $e=0.87 \mathrm{y}$ su período es $P_{\text {orb }}=3.4$ años.

${ }^{3}$ Sin embargo, es importante notar que hay MQs donde el objeto compacto es una estrella de neutrones, como Sco X-1 y Cir X-1.
} 
bien otro, donde el objeto compacto no es capaz de frenar la acreción y ocasionalmente impulsa parte del material que cae sobre él, formando chorros a lo largo de su eje de rotación. Como se mencionó en la Introducción, estos sistemas se denominan microcuásares. Les dedicaremos el capítulo siguiente mientras que en las próximas secciones se pretende ampliar lo comentado sobre los otros modelos actualmente populares para las binarias de rayos $\gamma$, concentrandonos en el caso de sistemas de gran masa. El Capitulo 10 trata el caso de un pulsar altamente magnetizado con acreción.

\subsection{Vientos en estrellas de gran masa}

Nos referimos aquí a las estrellas con tipos espectrales O, B y Wolf-Rayet (WR), estrellas de gran masa $\left(>20 \mathrm{M}_{\odot}\right)$ y altas luminosidade $\Psi^{4}\left(10^{5}-10^{6} \mathrm{~L}_{\odot}\right)$ con la presencia característica de líneas de emisión (por lo que se agrega una letra e al tipo espectral si se trata de las $\mathrm{OB})$.

Las estrellas Be muestran velocidades de rotación más alta que las B (del orden del $\sim 80 \%$ de la velocidad de rotación crítica) y se han observado en ellas variaciones en la intensidad de las líneas de Balmer y en el flujo contínuo y de líneas en el ultravioleta (UV). Dichos cambios se explican como variaciones en el aspecto de una envoltura de material circumestelar (ver ej. monografía de Porter \& Rivinius, 2003). En particular, una envoltura más fría que la estrella sería responsable del exceso en la emisión infrarrojo (IR) por sobre el valor fotósferico normal y las líneas de emisión en el visual. El proceso mediante el cuál se expele materia de la estrella no está del todo claro. Algunas propiedades han sido inferidas mediante estudios estadísticos de los perfiles de emisiones y exceso IR de estrellas individuales. La existencia de una estructura achatada (similar a un disco confinado cerca del plano ecuatorial) alrededor de las estrellas Be parece ser un punto bien establecido. Observaciones polarimétricas y utilizando interferometría de hecho han permitido detectar el disco de $\zeta$ Tauri (Quirrenbach et al. 1993). El modelado de estos discos requiere del conocimiento de propiedades físicas de la envoltura. Waters et al. (1987) han estimado densidades electrónicas del orden de $10^{-12}-10^{-11} \mathrm{~g} \mathrm{~cm}^{-3}$ en las regiones internas del disco, junto con perfiles de densidad con dependencia del tipo $r^{-n}$ siendo $2<n<4$. Actualmente, la teoría más aceptada para el modelado de estos discos es la que incorpora la viscosidad como mecanismo para el transporte de momento angular, de modo que el perfil de velocidad acimutal en función de la distancia a la estrella se mantiene Kepleriano (Lee, Saio \& Osaki, 1991) lo cual es consistente con la evidencia observacional. Cálculos tridimensionales de estas estructuras, y de las ondas de densidad que pueden darse en ellas han sido realizados por Okazaki (1991, y trabajos posteriores).

Por otro lado, se cree que el viento tendría además una componente menos densa y más rápida (supersónica, con velocidad terminal $v_{\infty} \sim 1000 \mathrm{~km} \mathrm{~s}^{-1}$ ), impulsada por la

\footnotetext{
${ }^{4}$ Comparable con la luminosidad de Eddington de la estrella, dada por $L_{\mathrm{Edd}}=4 \pi c G M_{\star} m_{\mathrm{H}} / \sigma_{T} \simeq$ $1.2 \times 10^{38}\left(M_{\star} / M_{\odot}\right) \mathrm{erg} \mathrm{s}^{-1}$.
} 
presión de radiación de absorciones discretas (líneas modificadas por efecto Doopler: en estas absorciones se transfiere el impulso que progresivamente acelera el viento hasta llegar al equilibrio que implica la velocidad terminal). Este viento sale de la estrella en forma preferentemente polar o isotrópica, y sería el responsable de la emisión UV. El perfil de velocidad es en este caso del tipo $v(r) \sim v_{\infty}\left(1-R_{\star} / r\right)^{\beta}$, siendo $\beta$ un parámetro del orden de la unidad (Lamers \& Cassinelli, 1999). Al estimar la tasa de pérdida de masa, para estrellas de tipo $\mathrm{OB}$ con luminosidad y temperatura efectiva respectivamente en los rangos $5<\log \left(L_{\star} / L_{\odot}\right)<6$, y $4.45<\log T_{\text {eff }}<4.7$, puede utilizarse una relación empírica: $\log \left(\dot{M} /\left(\mathrm{M}_{\odot} \mathrm{yr}^{-1}\right)\right)=1.738 \log \left(L_{\star} / L_{\odot}\right)-1.352 \log T_{\text {eff }}-9.543$, obtenida por Lamers \& Leitherer (1993).

Las tasas de pérdida de masa en las WR son las mayores que se han inferido, alcanzando $\sim 10^{-4} \mathrm{M}_{\odot} \mathrm{yr}^{-1}$ (Leitherer et al. 1997) . Las líneas en emisión de estas estrellas son particularmente anchas. Un repaso actualizado sobre las propiedades de las estrellas WR se dá en Crowther (2006). La secuencia de subtipos espectrales de las WR parece corresponder a estados evolutivos (breves) de las estrellas O. La aceleración de partículas y su consecuente emisión no térmica en el entorno de estas estrellas fue considerada por White (1985). Existe evidencia observacional de que los vientos de estrellas $\mathrm{O}$ y WR presentan inhomogeneidades de pequeña escala o grumos (clumps), por lo que el viento se caracteriza mediante un factor de llenado $f \sim 0.05-0.25$ (que dá un factor 2 a 4 menor en la tasa de pérdida de masa respecto a modelos homogeneos, $d M / d t \propto f^{1 / 2}$ ). Una vez que se han propagado, los vientos de estas estrellas interactúan con el material interestelar generando frentes de choque 5 socavando cavidades o burbujas (Benaglia \& Cappa, 1999). Como las estrellas de gran masa evolucionan rapidamente suelen encontrarse cerca de su lugar de formación (ej. formando asociaciones estelares, que también han sido estudiadas como posibles fuentes de rayos $\gamma$, Torres et al. 2004).

Hemos comentado algunos aspectos de la emisión y el viento de la estrellas de gran masa. A la hora de modelar la emisión generada en sus inmediaciones, los campos de materia, de fotones y magnético tienen estructuras complejas, para las cuales se adoptan descripciones simplificadas. El campo magnético en la superficie $\left(B_{\star}\right)$ de estrellas de gran masa se estima que fluctúa entre unos pocos Gauss y $\sim 10^{3} \mathrm{G}$ (Donati et al. 2002). La geometría del campo ha sido considerado por varios autores (White 1985; Lamers and Cassinelli 1999 y sus referencias) y puede representarse basicamente como la de un rotor. La velocidad de rotación de la estrella suele ser $v_{\star} \cong(0.1-0.2) v_{\infty}$ (Conti \& Ebbets 1977). Cerca de la estrella el campo magnético es aproximadamente radial, mientras que al alejarse se torna tangencial. Bajo la hipótesis de un viento estelar "caótico" las fluctuaciones del campo magnético pueden asumirse $\delta B / B \approx 16$.

\footnotetext{
${ }^{5}$ En estos choques entre el viento de estrellas de gran masa aisladas y el medio interestelar se ha estimado que se genera una luminosidad de $\sim 10^{32}-10^{33} \mathrm{erg} \mathrm{s}^{-1}$ a energías $>100 \mathrm{MeV}$ (Benaglia et al. 2001) que es demasiado bajo para ser detectable con los instrumentos actuales.

${ }^{6}$ En consecuencia, la difusión de partículas cargadas en el viento se dá en las condiciones de Bohm
} 


\subsection{Pulsares}

Es mucho lo que puede decirse sobre estos interesantes objetos, mientras que este espacio es obviamente limitado. El lector puede dirigirse por ej. a Lipunov (1992), y Shapiro \& Teukolsky (1983) para una introducción más amplia.

Poco después del descubrimiento de los radio pulsares en 1967 se los identificó con estrellas de neutrones magnetizadas, aisladas (sin compañera binaria) y rotando a altas velocidades. Sólo este tipo de objetos serían capaces de explicar los períodos cortos y estables de los pulsos observados, además de la alta polarización de su emisión en radio. Desde entonces se los ha detectado emitiendo en todo el espectro, hasta inclusive rayos $\gamma$ de altas energías $(\mathrm{MeV}-\mathrm{GeV})$. El eje magnético de la estrella de neutrones y su eje de rotación deben estar desalineados para justificar los pulsos. La radiación queda contenida en dos conos angostos a lo largo del eje magnético y el observador la detecta sólo cuando un cono apunta en dirección de la visual. La energía radiada se extrae de la energía cinética rotacional de la estrella de neutrones, que en consecuencia frena su rotación y aumenta el período de los pulsos.

La región inmediata del pulsar se llama su magnetósfera. En esta región, resulta una buena aproximación considerar al pulsar como un dipolo magnético (rotante, no alineado). Se llama radio de corrotación, o del cilindro de luz, a la distancia a la cual la velocidad de partículas que rotaran en forma rígida con la estrella de neutrones igualaría la velocidad de la luz. Dentro de ese radio las partículas cargadas están ligadas a las líneas de campo magnético. En el interior del cilindro de luz las líneas son cerradas, pero las que se extienden más allá del radio de corrotación son abiertas y, visto desde arriba, son arrastradas en forma de espiral.

De acuerdo al modelo de Goldreich \& Julian (1969), justo fuera de la superficie de la estrella de neutrones la fuerza de Lorentz sobre las partículas cargadas excede enormemente la fuerza de atracción gravitacional, lo que produce una magnetósfera rica en partículas que son "arrancadas" de la superficie de la estrella. En las inmediaciones de la zona polar se desatan cascadas electromagnéticas (dominadas por radiación de curvatura) que llevan a aumentar el flujo de electrones y positrones (Sturrock, 1971). Las partículas que se mueven a lo largo de las líneas de campo abiertas escapan formando el viento del pulsar. Sin embargo la relación entre entre el viento y la magnetósfera presenta todavía importantes retos teóricos, como describe Michel (2004). La radiación gamma debida a un pulsar aislado y sus inmediaciones (PWN: PULSAR WIND NEBULA) puede tener origen en tres zonas con distintas condiciones físicas: la magnetósfera, el viento relativista, o la nebulosa externa que se forma en la región donde se frena el viento, como esquematiza la Figura 3.2

La emisión generada en la magnetósfera es la que se observa pulsar con el período rotacional de la estrella, si bien los mecanismos generan la emisión no son claros aún. En esta región interna las pérdidas energéticas del pulsar están dominadas por flujo de Poynting, mientras que por fuera del cilindro de luz domina la energía cinética de las

(White, 1985) 
partículas del viento: un plasma de electrones y positrones relativistas que emite fotones energéticos por dispersión Compton inversa. El factor de Lorentz de este viento se estima entre $10^{4}$ y $10^{7}$. El viento del pulsar eventualmente se frena al chocar con el remanente de supernova asociado (plerión) o con el medio interestelar, generando en esta zona lejana una nebulosa que emite rayos X térmicos y por proceso sincrotrón. Los aspectos generales de la emisión del pulsar de Crab y su nebulosa pueden derivarse en el contexto de este modelo.

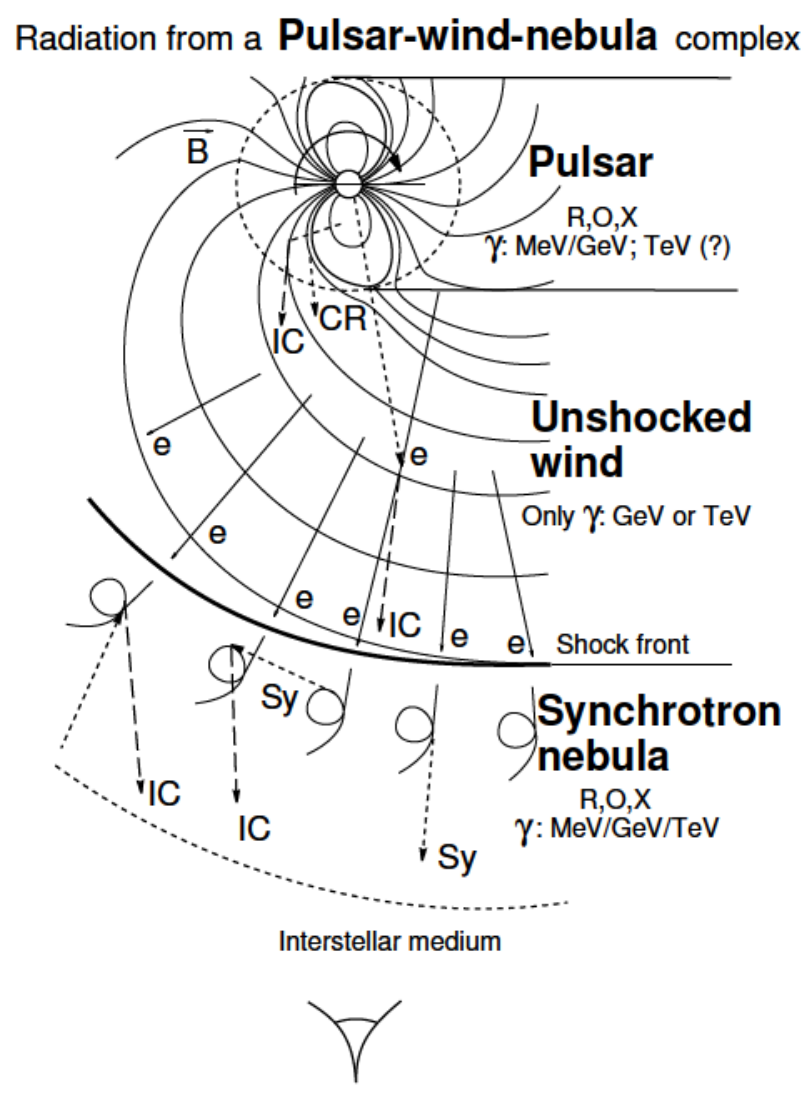

Figura 3.2: Esquema de las zonas de emisión gamma en torno a un pulsar, y los mecanismos radiativos que intervienen. Aharonian \& Bogovalov (2003)

\subsection{Sistemas tipo $\mathrm{WR}+\mathrm{OB}$}

Observaciones en radio han detectado la emisión no térmica que se genera en la zona de interacción entre los vientos de dos estrellas de gran masa que forman sistemas binarios. Cuando se tiene la suficiente resolución espacial (ej, utilizando VLA) la región emisora aparece como una región extendida de forma elongada entre las dos estrellas. Esta emisión proviene del enfriamiento (sincrotrón) de las partículas que han sido re-aceleradas en forma difusiva (ver Apéndice) en el frente de choque producto de la colisión de los vientos estelares. 
Este escenario fue investigado por Eichler \& Usov (1993) y luego por otros autores que implementaron modelos simplificados en el intento de mantener el cálculo analítico y poder estimar el amplio espectro emitido, que se extiende hasta las altas energías.

Asumiendo que el viento de ambas estrellas, primaria (subíndice 1, la WR) y secundaria (subíndice 2, la OB) fluye en forma radial y colisiona a la distancia $r_{i}$ de la estrella $i$, igualando presiones ram se obtiene $r_{i}$ en función de la separación de la binaria y del parámetro $\eta$, dado por $\eta=\left(\dot{M}_{2} v_{\infty, 2}\right) /\left(\dot{M}_{1} v_{\infty, 1}\right)$, donde también deben asumirse valores de las tasas de pérdida de masa $(\dot{M})$ y velocidades terminales $\left(v_{\infty}\right)$. Expresiones detalladas para la forma del frente de choque pueden hallarse en Antokhin et al. (2004) y sus referencias. La emisión de las partículas re-aceleradas puede calcularse dados los valores del campo magnético y de fotones (estelares) que se asuman en $r_{i}$. Más tarde, se han desarrollado modelos bidimensionales hidrodinámicos que permiten incorporar las distribuciones de presión y densidad de los vientos, llevando a una mejor estimación de la distribución espacial de las partículas re-aceleradas (Dougherty \& Pittard, 2006).

Van der Hucht (2001) lista 227 estrellas WR conocidas hasta ese momento, de las cuales, señala que el $\sim 40 \%$ forman sistemas binarios. Las binarias más conocidas cuya emisión $\gamma$ ha sido modelada son WR140 (que está en el borde de la caja de error de 3EG J2022+4317), WR146 y WR 147. En el caso de sistemas con excentricidad elevada, como el que se muestra en la Figura 3.3 la región de colisión de vientos sufrirá cambios importantes debido a la variación de la separación de las estrellas con el movimiento orbital. La binaria WR20a (el sistema binario de mayor masa en la galaxia) ha llamado la atención por encontrarse dentro de una región extensa detectada por HESS, en el cúmulo estelar abierto Westerlund 2 (Aharonian et al. 2007b). Bednarek (2005) ha trabajado sobre la propagación de los rayos $\gamma$ en WR20a, y la posible emisión de neutrinos por este sistema.

Si bien se ha prestado mayor atención a este tipo de sistemas por su emisión en rayos $\mathrm{X}$ (donde a veces es indistinguible la componente términa de la que no lo es), Benaglia \& Romero (2003) estimaron que la emisión de sistemas tipo $\mathrm{WR}+\mathrm{OB}$ a energías mayores que $100 \mathrm{MeV}$ puede variar entre los $10^{32} \mathrm{y}$ hasta $\sim 10^{35} \mathrm{erg} \mathrm{s}^{-1}$.

\subsection{Sistemas con colisión de viento pulsar-estrella}

En el entorno de pulsares aislados en movimiento respecto al medio, la interacción del viento del pulsar con el material interestelar produce un choque con frente en forma de arco (bow) en dirección opuesta a la del vector velocidad relativa. Un pulsar que interactúa con el viento de una compañera estelar de gran masa (sistemas tipo Be + PSR) está confinado por una presión intensa, y sólo si es capaz de generar un viento con la energética necesaria

\footnotetext{
${ }^{7}$ Para describir el viento del pulsar, se introduce un parámetro $\sigma$ llamado magnetización. Es el cociente entre densidades de energía magnética y cinética (de las partículas del viento). Se asume $\sigma<1$, y además, los modelos de Dubus suponen que la fracción de energía llevada por iones es despreciable, mientras que los leptones son monoenergéticos, con factor de Lorentz $\gamma_{p}$.
} 

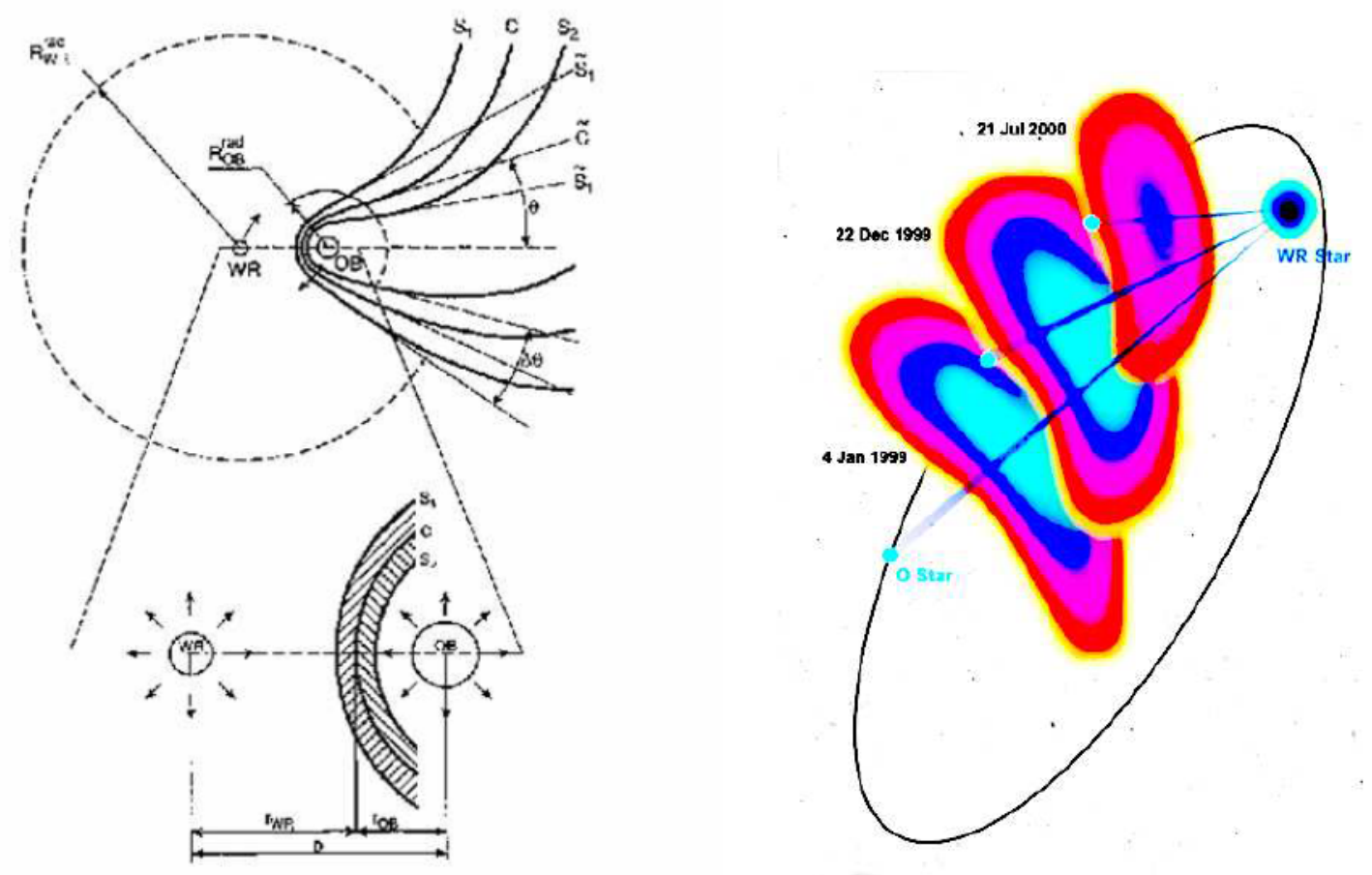

Figura 3.3: Sistemas con colisión de vientos, formados por dos estrellas de gran masa. A la izquierda: la propuesta original de Eichler \& Usov (1993). A la derecha se muestra un montaje de observaciones de WR140 obtenidas con VLA a $8.4 \mathrm{GHz}$, en tres posiciones orbitales. Puede verse la rotación de la zona de colisión de vientos (Dougherty \& Pittard, 2006).

podrá evitar la acreción. Por ejemplo si el viento de la estrella está caracterizado por $\dot{M}_{\mathrm{w}} \mathrm{y}$ $v_{\infty}$, la separación orbital es $a$, y la potencia cinética del viento del pulsar es $\dot{E}$; un modelo simplificado (Dubus, 2006a) donde se igualan presiones para despejar la distancia $R_{s}$ desde el pulsar donde se ubica el frente de choque (standoff distance), lleva a

$$
\frac{\dot{E}}{4 \pi R_{s}^{2} c}=\rho_{w} v_{\infty}^{2} \quad \rightarrow \quad R_{s}=\frac{a}{1+\left(\dot{M}_{\mathrm{w}} v_{\infty} c / \dot{E}\right)^{1 / 2}}
$$

donde se usó $\rho_{w}=\dot{M}_{\mathrm{w}} /\left(4 \pi v_{\infty}\left(a-R_{s}\right)^{2}\right)$ para la densidad del viento estela1 8 . Se obtiene para pulsares jóvenes (en rotación con período de milisegundos y tasa de frenado $\dot{P} \sim 10^{-15}$ ), con una potencia en el viento $\dot{E} \sim 10^{36} \mathrm{erg} \mathrm{s}^{-1}$ y parámetros similares a LS 5039, que $R_{s}$ es de aproximadamente una décima de la separación orbital.

Se ha sugerido que el frente de choque entre estos vientos tiene forma "cometaria" en dirección opuesta a la del vector pulsar-estrella, mostrando cambios notorios de orientación durante fases orbitales próximas al periastro. Como se ha dicho, la reaceleración de partículas en frentes de choque lleva a la emisión de radiación en un amplio rango de frecuencias.

\footnotetext{
${ }^{8}$ Me llama la atención que tiene solución $R_{s}<a$ independientemente de la desigualdad entre $\dot{E}$ y $\dot{M}_{\mathrm{w}} v_{\infty} c$.
} 


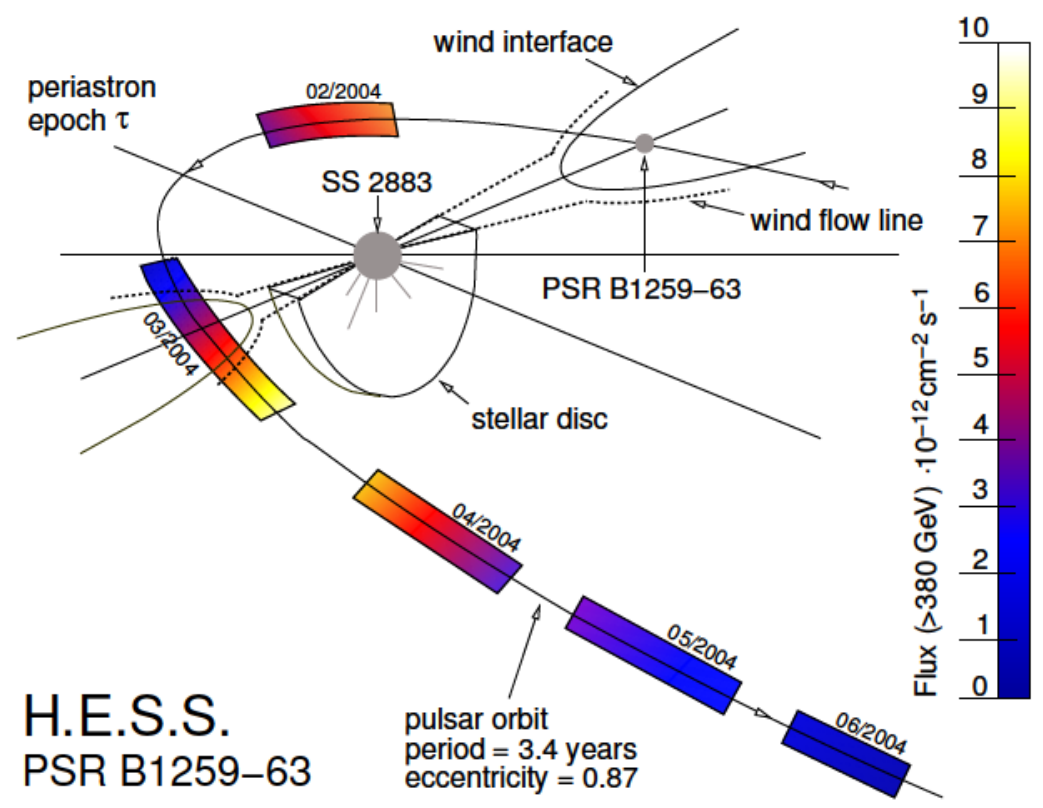

Figura 3.4: Esquema del sistema binario de PSR B1259-63 (Aharonian et al. 2005b). Se indica la posición que se ha estimado para el disco de la estrella Be. Las barras a lo largo de la órbita indican los períodos observados por HESS.

Además, un sistema de este tipo brinda un laboratorio muy interesante para el estudio del viento de pulsares, brindando un versión compacta de una PWN: el viento del pulsar es frenado aquí a una distancia $R_{s} \sim 10^{12} \mathrm{~cm}$. Otro hecho interesante es que la re-aceleración ocurre en presencia de un campo magnético más intenso $(B \sim 0.1-1 \mathrm{G})$.

El sistema PSR B1259-63/SS2883 es la primera confirmación de que este escenario dá lugar a emisión de rayos $\gamma$ de muy altas energías $(>380 \mathrm{GeV})$. Fue detectado por HESS (Aharonian et al. 2005b) mostrando un mínimo en su curva de luz cerca del pasaje por el periastro (cuando se prevee un incremento de la emisión por enfriamiento IC de leptones, pero también un aumento en absorción $\gamma \gamma$ ) y máximos alrededor de 20 días antes y después del mínimo. El trabajo de Khangulyan et al. (2006) prevee características específicas de los modelos teóricos que se han presentado para esta fuente. Es interesante comentar que del eclipse de las pulsaciones en radio se ha podido inferir la posición del disco circumestelar de la estrella Be. Dubus (2006b) ha estimado la emisión en radio de PSR B1259-63, y sugiere que la "radio-cola cometaria" en sistemas Be+PSR puede ser confundida por su emisión extendida con la emisión de un jet, característico de sistemas tipo microcuasar. De este modo, se unificaría el escenario para las binarias de rayos $\gamma$. Sin embargo, como veremos más adelante, esta sugerencia está lejos de ser confirmada. 
Capítulo 3. Binarias de Rayos $\gamma$ 


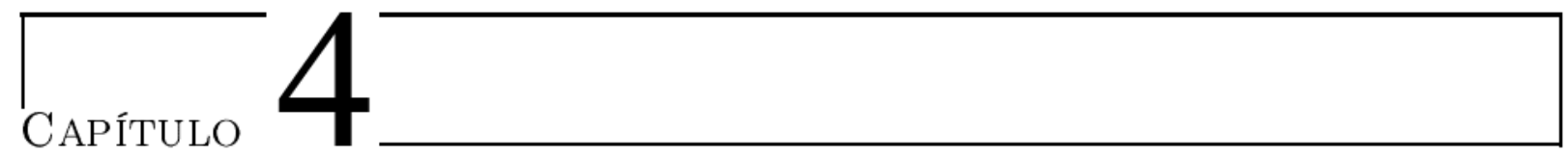

\section{Acreción y eyección de materia en binarias de}

\section{gran masa}

Durante el desarrollo de las investigaciones relacionadas con esta tesis se estudió la emisión de rayos $\gamma$ en sistemas conteniendo una estrella de gran masa y un objeto compacto. Este tipo de sistemas suele presentar episodios de emisión en rayos X (a veces emisión persistente, otras transitoria o "explosiva"), por lo que hablaremos con generalidad de HMXB. Los sistemas Be/X constituyen la mayor subclase de las HMXB, abarcando un $80 \%$ de ellas 1 . Nos referiremos a sistemas donde la emisión X está vinculada a la acreción de material del viento de la estrella normal sobre la compacta. Dicho proceso consiste en la acumulación de gas difuso o materia sobre un objeto, debido a la influencia gravitatoria que este ejerce en el medio donde está inmerso. Frank, King \& Raine (2002) brindan una monografía muy completa de este tema, central en la astrofísica.

El objeto compacto en los sistemas estudiados es una estrella de neutrones o un agujero negrd2. No hay razones para creer que las estrellas de neutrones que son las componentes ópticamente invisibles de fuentes binarias de radiación $\mathrm{X}$ sean de naturaleza diferente a las que puede observarse aisladas como pulsares en radio, y a las cuales nos hemos referido en el capítulo anterior. Si la estrella compacta tiene una masa mayor que $\sim 3$ $\mathrm{M}_{\odot}$, ésta es un agujero negro, y (sólo) nos interesó que se trata de una cierta cantidad de masa $M$ confinada dentro de una superficie esférica (su horizonte de eventos) con radio

\footnotetext{
${ }^{1}$ A unque los nuevos resultados de INTEGRAL en rayos $\mathrm{X}$ duros parecen haber cambiado esta relación, al encontrar muchos sistemas con estrella compañera de tipo supergigante, ver Dean et al. (2005).

${ }^{2}$ Las binarias candidatas a contener un agujero negro son comentadas por ej. en Casares (2005) y en Remillard \& McClintock (2006). Este último trabajo también introduce los fenómenos que pueden caracterizar a estos sistemas al tener campos gravitacionales fuertes, como ser el ensanchamiento relativista de líneas espectrales, y oscilaciones cuasi-periódicas de alta frecuencia $(100-450 \mathrm{~Hz})$. Ver Zhang, Li \& Wang (2004) para una discución sobre la falta de confirmaciones observacionales de sistemas Be/agujero negro.

Sobre recientes descubrimientos de enanas blancas con acreción, ver Mukadam et al. (2007), aunque aquí no se han contemplado este tipo de sistemas.
} 
$r_{\text {Sch }}=2 G M / c^{2}$, también llamado radio se Schwarzschild. Un agujero negro, a diferencia de una estrella de neutrones, no ofrece una superficie sólida contra la cual colisione el material acretado y este hecho puede, en principio, tener consecuencias observables.

El descubrimiento de fuentes intensas de rayos $\mathrm{X}$ en sistemas binarios cercanos fue el detonante de una nueva época en la astrofísica de altas energías cuando se estimó la eficicencia del proceso de acreción. De la conversión de energía potencial gravitatoria en energía cinética que mayormente se dirige a calentar el gas, se obtiene una luminosidad (de acreción) $L_{\mathrm{ac}}=\xi \dot{M} c^{2}$, siendo $\dot{M}$ la tasa de acreción de masa. Para una estrella de neutrones con masa $M=1.4 \mathrm{M}_{\odot}$ y radio $R=10 \mathrm{~km}$, resulta $\xi \sim 0.1$. Luego, la acreción sobre una estrella compacta es una fuente muy poderosa de energía, si tenemos en cuenta que la eficiencia energética en la nucleosíntesis que alimenta una estrella normal es sólo $\xi=0.007$ (Longair, 1992).

La fuente de energía de explosiones que se observan en rayos X es (indirectamente) la acreción. Para estrellas de neutrones con campos magnéticos débiles se ha propuesto que ocurre un proceso similar al que tiene lugar en ciertas variables cataclísmicas: material acretado acumulado sobre la superficie se enciende repentinamente en un flash termonuclear (ej. Joss \& Rappaport 1984). Los episodios de alta luminosidad en rayos X concuerdan muchas veces con modulaciones orbitales al darse la acreción sobre la estrella de neutrones en un sistema binario con gran excentricidad. Se ha establecido observacionalmente (Stella et al., 1986, Negueruela 1998) la siguiente clasificación de los estados de actividad X :

- Baja luminosidad persistente: la luminosidad en rayos $\mathrm{X}$ es $L_{X}<10^{36} \mathrm{erg} \mathrm{s}^{-1}$, o directamente no se detecta.

- Explosiones tipo I: $L_{X} \approx 10^{36}-10^{37} \mathrm{erg} \mathrm{s}^{-1}$, son explosiones que duran pocos días y su separación coincide con el período orbital del sistema. Se supone que ocurren cerca del momento de pasaje por el periastro.

- Explosiones tipo II o explosiones gigantes: $L_{X}>10^{37} \mathrm{erg} \mathrm{s}^{-1}$. No muestra modulaciones orbitales claras y puede durar hasta varias semanas.

Esta variedad de comportamientos indica una relación compleja entre la envoltura circunestellar de la primaria y el objeto compacto. Se ha propuesto que la pendiente del espectro en fuentes persistentes de rayos X, está relacionada con la detección del sistema en radio. Nos referimos a un modelo unificado en la Sección 4.3 luego de introducir los elementos básicos, a continuación.

\subsection{Discos de acreción en sistemas binarios}

Consideremos un sistema binario formado por una estrella compacta de masa $M$ y una estrella compañera que experimenta pérdidas de masa a través de su viento. Cuando la 
estrella compacta tiene un movimiento muy lento respecto al viento $\left(v_{\text {rel }} \ll c_{s}\right.$ donde $v_{\text {rel }}$ es la velocidad relativa entre el viento y la estrella compacta y $c_{s}$ es la velocidad del sonido en el viento), la acreción se dá mediante la caída libre de partículas del medio hacia la estrella y se habla de acreción esférica. Para establecer el radio de acreción $r_{\text {ac }}$ se igualan la energía cinética y potencial de una partícula, de modo que $r_{\mathrm{ac}}=2 G M / v_{\mathrm{rel}}^{2}$. Utilizando este valor para definir una sección eficaz de captura, si el medio tiene densidad $\rho_{\mathrm{w}}$, la tasa de acreción de masa resulta (Bondi \& Hoyle, 1944):

$$
\dot{M}_{\mathrm{ac}}=\pi r_{\mathrm{ac}}^{2} v_{\mathrm{rel}} \rho_{\mathrm{w}}=\frac{4 \pi(G M)^{2} \rho_{\mathrm{w}}}{v_{\mathrm{rel}}^{3}} .
$$

Esta expresión puede utilizarse para obtener estimaciones a primer orden, pues normalmente, el movimiento orbital evita que la masa que escapa de la secundaria caiga directamente sobre la estrella compacta. Si el radio de la primaria es alrededor del $5 \%$ de la separación de la binaria $a$, la corriente de gas en vez de caer directamente sobre la superficie de la estrella se mueve en orbita alrededor de esta formando un disco de gas caliente que tiende a coincidir con el plano orbital.

La viscosidad del gas, entendida como fricción interna que convierte el movimiento ordenado de las partículas en movimiento térmico, hace que las partículas pierdan energía al orbitar y lentamente caigan en trayectoria espiral hacia la estrella. El mecanismo físico responsable de la viscosidad en discos de acreción es pobremente entendido aún. Su efecto sin embargo es claro: calentar el gas a medida que desciende hasta que finalmente choca con la superficie de la estrella.

Una primera aproximación para el planteo del disco de acreción es suponer que se trata de un disco delgado, opticamente grueso que radía como cuerpo negro. Para que dicha aproximación sea válida debe darse el equilibrio hidrostático en la dirección $(z)$ perpendicular al disco,

$$
\frac{\partial p}{\partial z}=-\frac{G M \rho \sin \theta}{r^{2}} \Longrightarrow \frac{p}{h} \approx-\frac{G M \rho h}{r^{3}}
$$

donde $p$ es la presión, $h$ la mitad del espesor del disco y $\rho$ su densidad.

Aunque el gas esté cayendo lentamente hacia la estrella, se mueve en buena aproximación siguiendo órbitas circulares Keplerianas, por lo que $v_{\phi}{ }^{2} \sim G M / r$. Reemplazando aquí el valor de $G M / r$ que se obtiene de la expresión (4.2) y el valor de la velocidad local del sonido $c_{\mathrm{s}} \approx \sqrt{p / \rho}$, resulta

$$
\frac{h}{r} \approx \frac{c_{\mathrm{s}}}{v_{\phi}}
$$

Esta última cantidad es la inversa del número de Mach para la velocidad de rotación relativa a la velocidad local del sonido en el disco. Entonces, la condición para la validez de la aproximación de disco delgado es que la velocidad de rotación del disco sea mucho mayor que la velocidad del sonido. Esto equivale a decir que el gradiente de presión vertical no debe inflar el disco. Este hecho lleva también a que las temperaturas del disco deben 
ser bajas $\left(k T \ll G M m_{p} / r\right)$ de modo que el calor generado por los esfuerzos viscosos sea radiado eficientemente, y no acumulado por el disco (ver Shapiro \& Teukolsky 1983). La solución estacionaria a este problema fue hallada por Shakura \& Sunyaev (1973), quienes introdujeron la llamada "prescripción $\alpha$ ", comprendiendo en un solo parámetro toda la física sobre los procesos turbulentos y viscosos que ocurren en el disco. Explicitamente, se adopta

$$
\nu=\alpha c_{s} h, \quad \operatorname{con} \alpha \leq 1,
$$

siendo $\nu$ el coeficiente de viscosidad cinemática ${ }^{3}$. Este modelo para un disco de acreción es conceptualmente la solución más simple a las ecuaciones hidrodinámicas para la acreción en un disco delgado. Por supuesto, modelos más complejos son posibles.

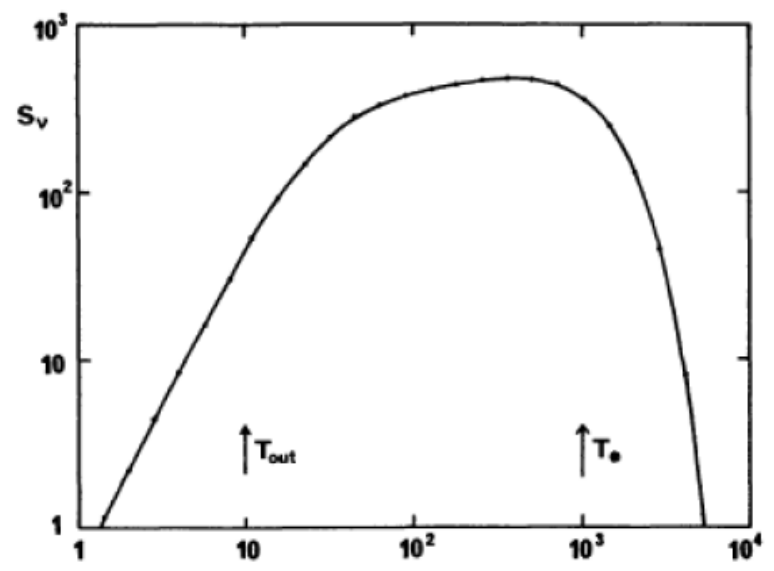

Figura 4.1: Espectro emitido por un disco opticamente grueso, y geometricamente delgado (Pringle, 1981), al que nos referimos como disco estándar. En las abscisas, el parámetro es la frecuencia y en el eje de ordenadas el flujo tiene unidades arbitrarias. Se indican las frecuencias correspondientes a las temperaturas de $R_{0}$ y $R_{\text {out }}$. Este espectro es similar al de un cuerpo negro "ensanchado".

La distribución de temperaturas en un disco estándar es independiente de la viscosidad y resulta $T(r) \propto r^{-3 / 4}$, con un valor máximo $T_{\text {eff }} \sim 10^{7} \mathrm{~K}$ para un agujero negro de masa estelar. La apariencia del espectro emitido puede verse en la Figura 4.1$]$ Se obtiene integrando la emisión de cuerpo negro $I_{\nu}=B_{\nu}[T(r)]$ de anillos situados entre los radio interno y externo del disco ( $R_{0}$ y $R_{\text {out }}$, respectivamente). El flujo en función de la frecuencia

\footnotetext{
${ }^{3}$ De este modo $\alpha$ es una cantidad media, promediada en la dirección perpendicular al disco, y sólo está bien definida para discos geometricamente delgados. Las propiedades del disco tienen una dependencia débil con el valor de $\alpha$ adoptado. Sin embargo, el valor de este parámetro que se infiere para explicar las observaciones es un orden de magnitud mayor que el que se estima a partir de simulaciones numéricas (King, Pringle \& Livio, 2007).
} 
$\nu$ para un disco con inclinación $i$ a una distancia $D$, resulta

$$
S_{\nu}=\frac{2 \pi \cos i}{D^{2}} \int_{R_{0}}^{R_{\text {out }}} I_{\nu} r d r= \begin{cases}\propto \nu^{2}, & \nu \ll k T\left(R_{\text {out }}\right) / h \\ \propto \nu^{1 / 3}, & k T\left(R_{\text {out }}\right) / h \ll \nu \ll k T\left(R_{0}\right) / h \\ \text { caída exponencial, } & \nu \ll k T\left(R_{0}\right) / h\end{cases}
$$

Expresiones para las cantidades físicas del disco en función de $r$ y para $M$ y $\dot{M}$ fijos se hallan, por ej., en Shapiro \& Teukolsky (1983). Cualitativamente, el disco estándar puede dividirse en tres regiones, dependiendo de $r$. Estas regiones son:

1. Una región exterior, para $r$ grandes, en la cual domina la presión de radiación y la opacidad es controlada por absorciones "libre-libre".

2. Una región intermedia, para $r$ pequeños, donde domina la presión de gas y la opacidad se debe principalmente a la dispersión electrónica.

3. Una región interna, para $r$ muy pequeños, en la que domina la presión de radiación y el scattering continúa predominando en la opacidad.

La transición entre las regiones media e interna ocurre cuando la presión del gas es del orden de la de radiación. El borde interno del disco se determina según el tipo de estrella compacta: para un agujero negro de Schwarzschild, por condiciones de estabilidad de las órbitas por efectos relativistas es $R_{0}=6 G M_{\mathrm{bh}} / c^{2}$. Para una enana blanca (wd) o una estrella de neutrones (NS), el disco alcanza la superficie de la estrella y es $R_{0}=\mathrm{R}_{\mathrm{wd} / \mathrm{NS}}$. Sin embargo, el campo mágnetico de la estrella puede ser un factor determinante de la estructura del disco en sus regiones internas (ver Capítulo 10).

El estudio de fuentes de rayos $\mathrm{X}$ ha provisto algunas estimaciones empíricas del parámetro $\alpha$. Un valor considerado aceptable es $\alpha \sim 0.1$, con el cual se puede explicar el espectro de rayos X blandos en objetos compactos con acreción. Sin embargo, por sí solo este modelo sufre problemas al intentar modelar la emisión a energías ligeramente mayores (rayos X duros), detectada en algunos objetos, como por ejemplo Cyg X-1 (Figura 4.2). Observaciones de esta fuente motivaron la propuesta de una región interna con características diferentes a las del disco estándar.

\subsubsection{La corona}

Cuando la tasa de acreción es comparable con el valor crítico $\dot{M}_{\text {crit }}=L_{\text {Edd }} /\left(\eta_{\text {ef }} c^{2}\right)$, donde $L_{\text {Edd }}$ es la luminosidad de Eddington del objeto compacto y $\eta_{\mathrm{ef}}<1$ la eficiencia en la conversión de masa en radiación, la solución de disco geometricamente delgado no puede extenderse hasta el objeto compacto. Al contrario, en sus proximidades la presión de radiación hace crecer su espesor $h(r)$ hasta hacerlo comparable con $r$. Modelos posteriores han incorporado ad-hoc una región interna tipo corona donde se producen los rayos $\mathrm{X}$ duros, 
y mantienen para la parte externa un disco estándar. Para describir la corona se utilizan dos temperaturas: los electrones y los iones son tratados por separado. Los primeros se enfrían radiativamente con eficiencia dando una componente más fría que la de los segundos: temperatura iónica $\sim 10^{11-12} \mathrm{~K}$, temperatura electrónica $\sim 10^{9} \mathrm{~K}$ (Esin, McClintock \& Narayan 1997). La fuente de los rayos X duros es el proceso Compton inverso que sufren los electrones de la corona con fotones blandos de las partes externas del disco. La variabilidad del flujo de rayos $\mathrm{X}$ duros en escalas temporales de hasta los milisegundos implica que la corona no puede extenderse más allá de unos cientos de $\mathrm{km}$, probablemente hasta unos $\sim 20-50 r_{g}$ desde el objeto compacto. Como puede verse en la Figura 4.2 la geometría del sistema condiciona fuertemente las características del espectro de emisión resultante.
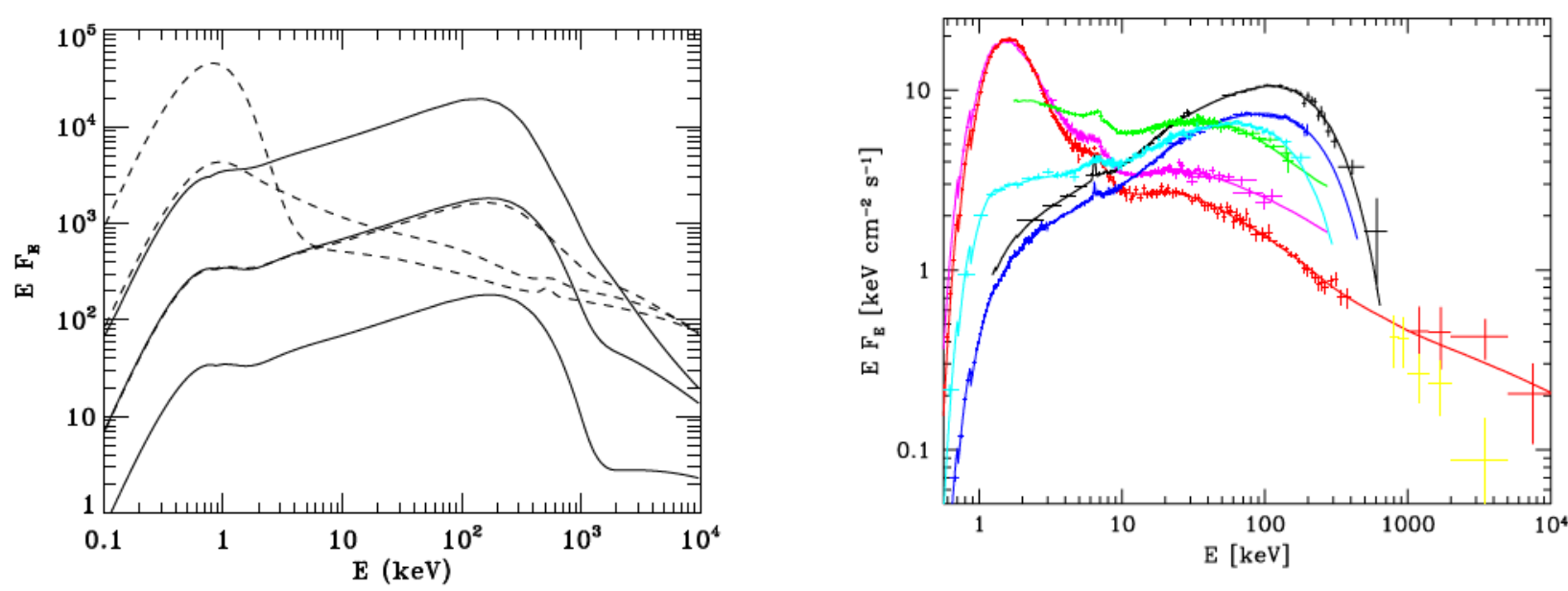

Figura 4.2: Izquierda: Modelos para la distribución espectral de energías en rayos X incluyendo disco y corona. Se han variado los parámetros que determinan los tamaños relativos de estas componentes, y la temperatura de las fases (electrones/iones) que componen la corona. La opacidad del plasma introduce una complejidad adicional (Poutanen, 1998). Derecha: Una serie de observaciones de banda ancha, ilustran la variedad de estados espectrales de Cyg X-1 (Zdziarski et al. 2002).

Como alternativa a modelos de tipo disco-corona, se han planteado modelos que incorporan un término de advección en las mismas ecuaciones hidrodinámicas del disco estándar (Ichimaru 1977, Narayan \& Yi 1994, 1995). Por su sigla en inglés se conoce a estas soluciones como modelos ADAF (Advection Dominated ACCRetion Flow). El gas acretado tiene en este caso una densidad baja, por lo que es opticamente delgado y se enfría en forma poco eficiente. La energía liberada por la fricción viscosa es almacenada como energía interna (aumentando la temperatura del gas) en vez de ser radiada, y resulta advectada hacia el objeto central4, junto con el material acretado. Geometricamente, la región ADAF es cuasi-esférica, y al igual que en los modelos de corona, la emisión de fotones $\mathrm{X}$ duros se produce por comptonización. Como ventaja sobre el modelo de corona, las soluciones

\footnotetext{
${ }^{4} \mathrm{O}$ bien es redireccionada hacia flujos salientes o jets.
} 
tipo ADAF son más estables, y se aplican con las tasas de acreción de masa $\dot{M}$ menores, típicos de regimenes sub-Eddington.

\subsubsection{Transferencia de masa:}

Trabajos recientes (ej. Neguerela \& Okazaki, 2001, y otros trabajos por Okazaki y colaboradores) han considerado los efectos sobre el disco ecuatorial de gas en torno de una estrella Be que, junto con un objeto compacto, forma un sistema binario. Dicho disco provee un medio más denso para la acreción que la componente de viento con simetría esférica, y puede por tanto llevar a tasas de acreción de masa mucho mayores. La interacción es compleja y se analiza recurriendo a simulaciones numéricas dinámicas que aplican el método SPH (Smoothed Particle Hydrodynamics) tridimensional, adoptando la prescripción $\alpha$. La idea central es que la fuerza de marea del objeto compacto ejerce un torque negativo sobre el disco de decreción de la estrella Be, disminuyendo la acción del torque viscoso más allá de cierto radio crítico, lo que lleva al truncamiento del disco circunestelar. La materia se acumula en anillos externos hasta que se desatan oscilaciones globales en la densidad del disco, que forman concentraciones en forma de brazo espiral6. Cuando estas concentraciones alcanzan al objeto compacto se incrementa tanto la tasa de acreción que probablemnete se observen explosiones en rayos X. Por otro lado si el truncamiento no es eficiente o si el disco de decreción supera el radio del lóbulo de Roche en fases cercanas al periastro, pueden observarse explosiones de rayos X cuasi-periódicas (tipo I). Esquematicamente, esta interacción de transferencia de masa se resume en la Figura 4.3 Para un rango amplio de parámetros orbitales, el truncamiento del disco estelar ocurre a un radio menor que el de la distancia al objeto compacto durante el periastro, y la tasa de acreción resulta fuertemente dependiente con la fase orbital, siendo basicamente sensible a la excentridad del sistema y a la inclinación entre el disco circunestelar y el plano orbital. Retomaremos este tema al presentar los resultados de simulaciones dinámicas del sistema LS I +61 303.

\subsection{Jets y microcuásares}

Eyecciones colimadas de materia han sido observadas en diversos objetos astrofísicos: estrellas muy jóvenes, algunas binarias con emisión en rayos $\mathrm{X}$, naciendo en el núcleo de galaxias activas, y se cree que se formarían transitoriamente en explosiones de supernova y GRBs. Estos objetos comparten características comunes: los chorros de partículas que llamaremos, como en inglés, jets, forman conos de pequeña apertura y su presencia se correlaciona con un disco de acreción rodeando a la masa central, en tanto que la potencia

\footnotetext{
${ }^{5}$ Ver en Frank, King \& Raine (1992) discusión sobre la inestabilidad viscosa-térmica de Lightman \& Eardley (1974).

${ }^{6}$ Perturbaciones de este tipo eventualmente se disiparán, por lo que se espera observar ciclos de regeneración del disco estelar. La evolución de los discos de acreción y decreción es tratada en Hayasaki \& Okazaki (2006). Se esperan variaciones observables en la línea $\mathrm{H} \alpha$.
} 
total en un jet es una fracción de la potencia vinculada con la materia acretada. Se han observado tanto jets relativistas como otros que no lo son. Para los del primer tipo, se vuelven importantes los efectos de la relatividad especial y cualquier emisión tiende a estar colimada en la dirección del flujo. Normalmente, se describe la velocidad de un jet mediante su factor de Lorentz macroscópico

$$
\Gamma=\left(1-\frac{v^{2}}{c^{2}}\right)^{-1 / 2},
$$

donde $c$ es la velocidad de la luz, y $v$ la del fluido. Otro parámetro relacionado a éste es el factor de Doppler $D$, dado por

$$
D=\left[\Gamma\left(1-\frac{v}{c} \cos \theta\right)\right]^{-1}
$$

siendo $\theta$ el ángulo que forma el eje del jet con la línea de la visual. Este factor ayuda a describir los efectos de magnificación Doopler y enfocamiento (beaming): un jet que se acerca al observador parece emitir a frecuencias $D$ veces mayores, y dentro de un ángulo sólido $D^{2}$ veces menor que en el sistema de referencia co-móvil al jet. Un factor $D^{3}$ entra entonces en el brillo aparente y un jet que se acerca al observador resulta más brillante. Lo inverso ocurre para un jet que se aleja, cuyo brillo aparecerá debilitado.

Nos dedicamos aquí a los jets de binarias de rayos $\mathrm{X}$, ya que nos interesa analizar luego a estos sistemas como binarias de rayos $\gamma$. Sistemas binarios con emisión X y para los que se ha detectado, en alguna ocasión, la presencia de jets, se llaman micocuásares (para los que mantendremos la sigla en inglés, MQ: MiCRO-QUASAR). Este nombre fue adoptado por Mirabel et al. (1992) en alusión a la clara similitud que presentan algunos de los aspectos básicos de los cuásares extragalácticos con los MQs: la presencia de un objeto compacto central, un disco de acreción y jets relativistas. Existe realmente un factor de

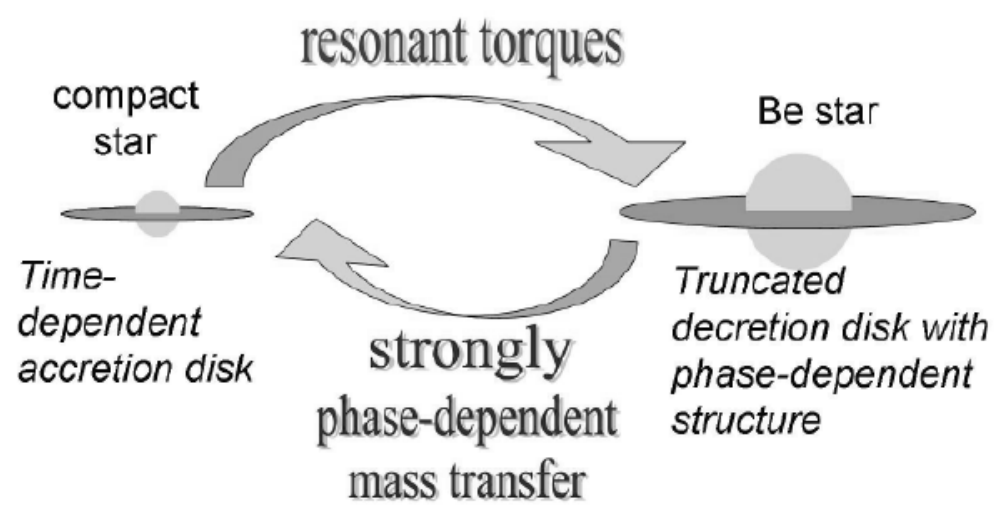

Figura 4.3: Esquema de la relación entre los discos de acreción y de decreción en sistemas BeX, sugerido por los resultados de simulaciones dinámicas (Okazaki, 1991). 
orden $\sim 10^{5}-10^{7}$ entre las masas centrales en estas dos clases de objetos, que se cree son manifestaciones de procesos físicos subyacentes muy parecidos. Las escalas temporales en las cuales se manifiestan variaciones que permitan estudiar tales procesos son del orden de horas a meses en MQs, haciéndolos blancos mucho más atractivos para el estudio que los cuásares donde las escalas temporales pueden ser de miles de años.

El origen de las eyecciones se ha convertido en un campo de investigación muy activo. Blandford \& Payne (1982) fueron los primeros en obtener el cálculo completo para la relación entre el flujo de plasma (electrones y protones) y el campo magnético llevado por el jet, mostrando los rasgos necesarios: colimación y aceleración tal que permita los factores de Lorentz observados. Como ya era sospechado, se hace necesaria la aplicación cuidadosa de la teoría magnetohidrodinámica 8 para el estudio de los jets, considerando además el campo magnético generado por el disco de acreción. Se han implementado simulaciones numéricas (ej. Meier et al. 2001, Zanni et al. 2007 y sus referencias) e intentado soluciones semi-analíticas (ej. Hujeirat et al. 2003). Además, se han considerado otras opciones para el confinamiento lateral de los jets, como por ej. el confinamiento por un plasma frío (BoschRamon et al. 2006a) o bien que el movimiento sea mucho más rápido $(\Gamma>10)$ de lo usualmente inferido $(\Gamma \sim 2-5$.) y son efectos puramente relativistas los que hacen verlos como colimados (Miller-Jones et al. 2006).

El principal aspecto observacional para la identificación de jets en MQs es su emisión en radio, que presenta un espectro no-térmico y un alto grado de polarización lineal. Estos dos rasgos revelan el origen de la emisión como el resultado de la interacción de una población de electrones relativistas con un campo magnético. El espectro plano resulta de superponer un número de espectros simples de emisión sincrotrón generados a lo largo de un flujo cónico, donde el plasma emisor se vuelve más opticamente delgado en las bajas frecuencias a medida que avanza alejándose. Dado que sólo la emisión sincrotrón, proveniente del contenido leptónico, se ha detectado en los jets de binarias de rayos X, existe muy poca información acerca de su contenido bariónico. Aunque esta afirmación no es general: la detección de líneas ópticas, en IR y rayos X en el sistema SS433 ha permitido inferir la presencia de iones en su jet (Margon 1984, y ver Kotani et al. 1994).

Cotas a la cantidad de energía total transportada por los jets $(\dot{E} \times$ tiempo de vida) pueden inferirse de su interacción con el medio interestelar que rodea al sistema. Un caso típico en este sentido es el de SS433: la nebulosa W50 rodea a este MQ y hace de calorímetro a la potencia mecánica de sus jet. Mediante observaciones en radio se ha concluido que esta puede ser tan grande como $\sim 10^{39} \mathrm{erg} \mathrm{s}^{-1}$ (Begelman, King \& Pringle, 1980). Los jets de Cyg X-1, con una potencia mecánica similar a su luminosidad bolométrica en rayos X?,

\footnotetext{
${ }^{7}$ Las temperaturas de los discos de acreción también tienen varios ordenes de magnitud de diferencia: en los cuásares emiten radiación óptica mientras que en MQs, en rayos X.

${ }^{8}$ En particular se suele asumir el límite de la MHD ideal: el plasma es cuasi-neutro y se lo trata como un fluido con conductividad infinita, en el cual están congeladas las líneas de fuerza. La tensión magnética es la responsable (como en un "pinch") de colimar el flujo.

${ }^{9} \mathrm{El}$ valor antes indicado es muy superior al que sugiere el brillo de los jets, y es por esto que suele hablarse de "jets oscuros".
} 


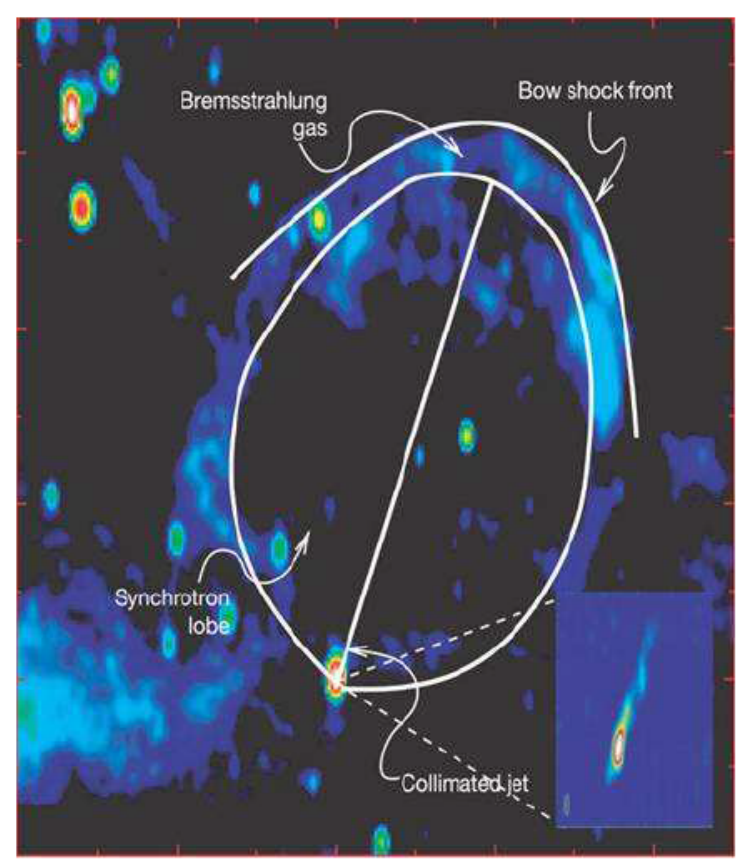

Figura 4.4: Efectos sobre el medio interestelar cercano a Cyg X-1: Observación a $1.4 \mathrm{GHz}$ por Gallo et al. (2005). El recuadro muestra una ampliación del jet de este microcuásar de gran masa. Esta imagen pone en evidencia la enorme potencia mecánica de los jets.

han "inflado" arcos cuya emisión se extiende muy lejos de la fuente, formando una especie de anillo de 5 pc de diámetro (Gallo et al. 2005), que también ha sido detectado en el óptico (Russell et al, 2007). Otro sistema donde se observa la interacción de los jets con el medio que los rodea es Cir X-1 (la LMXB cuyos jets tienen el mayor factor de Lorentz estimado a la fecha), ver Tudose et al. (2006).

\subsection{Estados espectrales: hacia un modelo unificado}

El sistema GRS $1915+105$ ha jugado un papel importante en el entendimiento de la relación entre los procesos de acreción y eyección en MQs. En 1997, una campaña de observaciones multifrecuencia ha permitido vislumbrar dicha relación (Mirabel et al. 1998). De la Figura 4.5 puede inferirse la desaparición de la parte interna del disco de acreción, puesta en evidencia por la disminución del flujo de rayos X. A continuación se eyecta una nube (plasmón) relativista que corresponde a la variación en IR cercano y luego en radio, al irse volviendo la nube más opticamente delgada. Un análisis de la variación en los flujos y pendientes espectrales (dureza) sugiere que es parte de la corona (emisora en rayos X-duros) la que es eyectada durante el evento.

Espectro en rayos X: Se conoce desde hace un par de décadas que la distribución espectral en energías de binarias de rayos $\mathrm{X}$ consiste de una componente térmica y una 


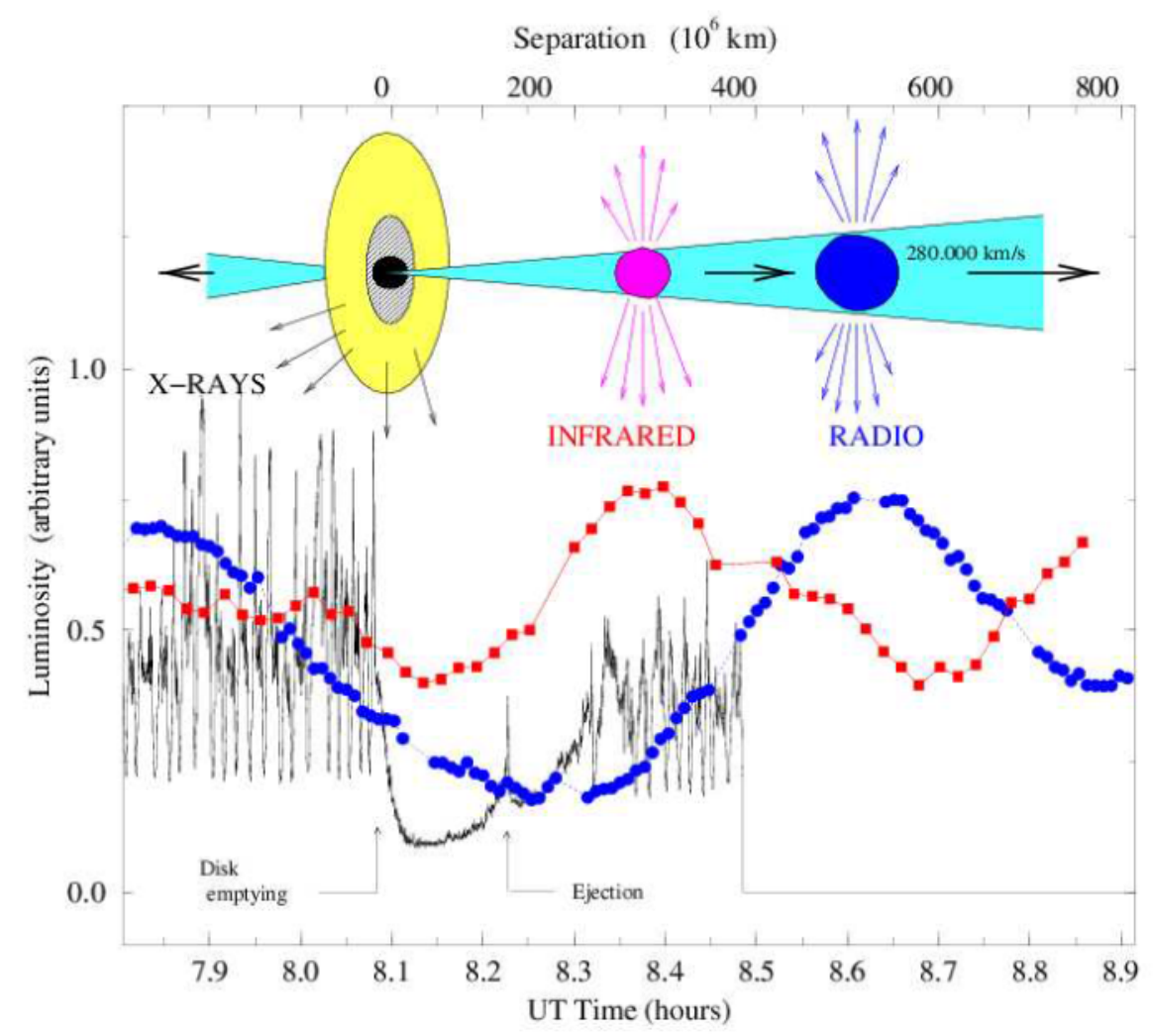

Figura 4.5: Las primeras evidencias de la relación entre acreción y eyección de material fueron inferidas del comportamiento multifrecuencia de GRS 1915+105 (Mirabel et al. 1998).

componente no-térmica10. Algunas binarias han mostrado transiciones en cuanto a cuál de estas componentes domina la luminosidad en la banda X. La componente térmica corresponde a la emisión de partes internas del disco de acreción con una temperatura del orden del keV, y cuyo espectro es similar al de la Figura 4.1. La componente no-térmica es usualmente modelada con un espectro tipo ley de potencia, y se caracteriza por un índice espectral $\alpha$ de modo que $n(E) \propto E^{-\alpha}$, donde $n(E)$ indica número de fotones por unidad de energía y volumen. La ley de potencia normalmente se extiende hasta energías de rayos $\mathrm{X}$ duros, $\mathrm{y}$ a veces muestra un quiebre o corte exponencial a energías mayores (en el rango gamma).

Un espectro de pendiente pronunciada (soft) en la banda X, es observado mayormente en fuentes luminosas y recibe el nombre de estado "high/soft". Fuentes con estas características espectrales en la banda $\mathrm{X}$ no son detectadas en radio. Por otro lado, espectros con una pendiente menor (índice $\alpha \sim 1.7$ ), suelen darse cuando las fuentes son más débiles, recibiendo el nombre de estado "low/hard". En este caso no se detecta emisión del disco o aparece mucho más frío y retirado respecto del objeto compacto. Suele hablarse de un

\footnotetext{
${ }^{10} \mathrm{~A}$ veces aparece también en el espectro la línea de emisión $\mathrm{Fe} \mathrm{K} \alpha$, a $\sim 6.4 \mathrm{keV}$.
} 
estado adicional, el "very high", caracterizado por la presencia de ambas componentes (térmica y no-térmica), pero con la ley de potencias mucho más pronunciada $(\alpha \sim 2.5)$ que en el estado hard. En este estado se suelen hallar rasgos de tipo QPO cuando se realiza un análisis temporal de la seña 11

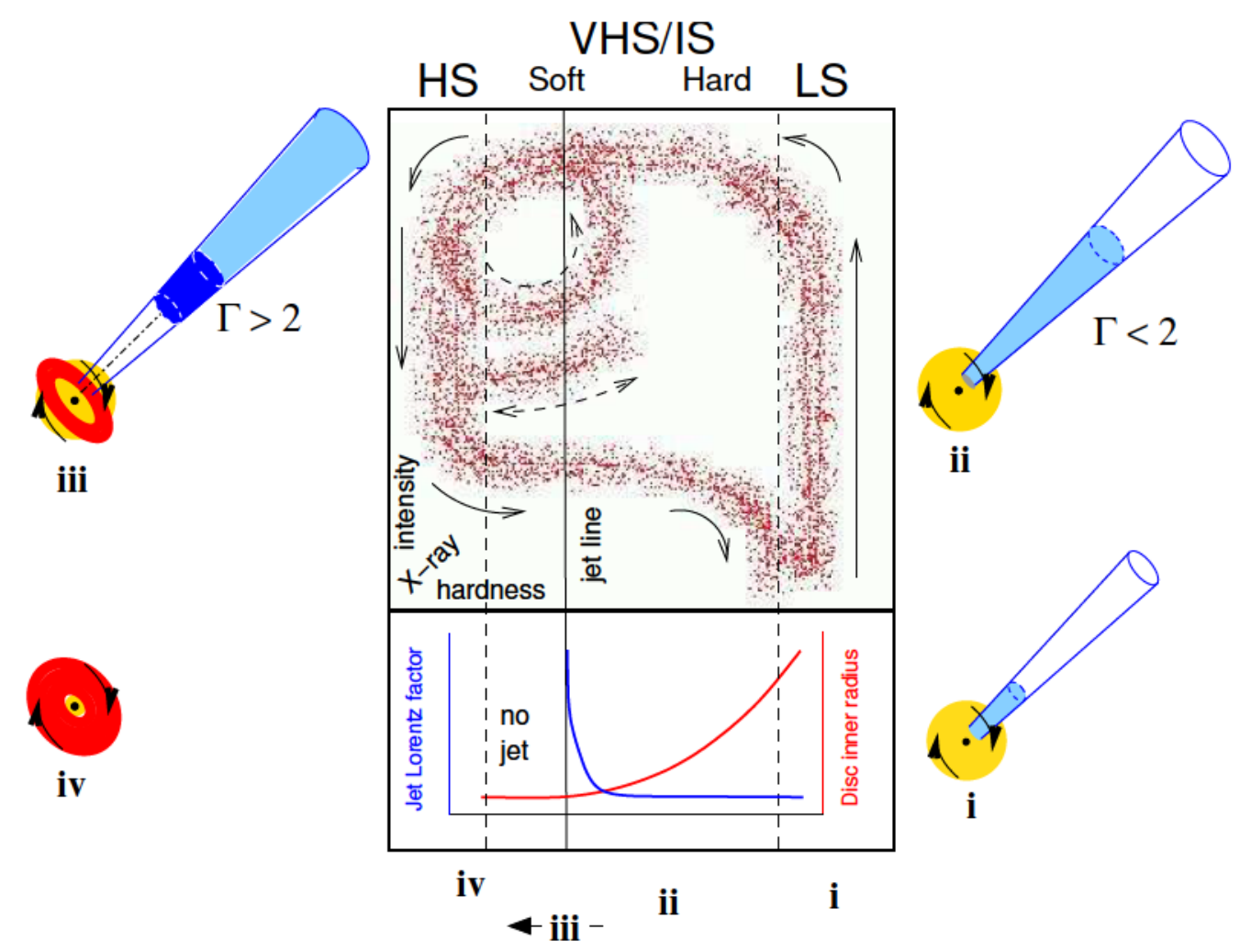

Figura 4.6: Esquema de la evolución en un diagrama dureza/intensidad del ciclo de estados para una binaria de rayos X conteniendo un agujero negro, Fender et al. (2004). El tiempo progresa en sentido antihorario, comenzando por la esquina inferior derecha (ver detalles en el texto). Los gráficos laterales ilustran la importancia de las distintas contribuciones: jets (azul), corona (amarillo) y disco de acreción (rojo).

Observaciones en radio han provisto argumentos en favor de la asociación entre el estado low/hard y la presencia de jet compactos y prácticamente estacionarios (Fender 2006). La evidencia incluye:

i. La presencia de jets en escalas de mili-segundo de arco ( decenas de UA), detectadas en imágenes de radio VLA y VLBA de algunas fuentes

\footnotetext{
${ }^{11}$ Quizás una descripción cuantitativa más útil de los estados espectrales es la sugerida por McClintock \& Remillard (2006), que basados en la revisión de numerosas observaciones obtenidas con RXTE concluyen que es más conveniente y consistente describir el espectro utilizando la función densidad de potencias del espectro (transformada Fourier), abandonando la luminosidad como criterio de clasificación. Estos autores sugieren hablar de estados steep power-law, thermal y hard.
} 
ii. Correlación entre las intensidades en $\mathrm{X}$ y radio, $\mathrm{y} / \mathrm{o}$ la presencia de un espectro bastante plano o invertido en radio, que permite inferir la presencia de jets cuando no se cuenta con imágenes de alta resolución.

iii. Y la frecuente inhibición de la señal persistente en radio que ocurre en la transición entre estados low/hard y high/soft.

Con respecto al item ii, Corbel et al. (2003) han relacionado las luminosidades en radio y rayos X, ajustando una ley empírica. En forma más reciente (incorporando datos de Chandra) se ha sugerido la siguiente relación entre las luminosidades de radio y X: $L_{R} \propto$ $L_{X}^{0.58 \pm 0.16}$, para $L_{X}$ en el amplio rango entre $10^{-8.5}$ y $10^{-2} L_{\text {Edd }}$ (Gallo et al. 2006).

En el esquema propuesto por Fender et al. (2004) se utiliza un diagrama de dureza del espectro (cociente del número de cuentas en dos bandas diferentes) versus intensidad, en rayos X (hardness-intensity diagram 12), para intentar brindar una explicación dinámica al "ciclo" de estados de binarias de rayos X cuyo objeto compacto es un agujero negro, en términos de las propiedades del jet (factor de Lorentz $\Gamma$ del jet persistente y de una nube eyectada), y los regimenes de acreción (radio interno del disco). La Figura 4.6 muestra el esquema propuesto. Inicialmente, el sistema está en un estado low/hard clásico, mostrando un jet persistente y moderadamente relativista $(\Gamma<2)$, que emite en radio con espectro plano. La luminosidad comienza a incrementarse en todas las frecuencias, manteniendo la dureza del espectro en rayos X, hasta alcanzar algun porcentaje de la luminosidad de Eddington (en rayos X). Una transición abrupta ocurre (linea horizontal superior), mientras se produce un destello en la banda radio, probablemente debido a un episodio de eyección repentino. El factor de Lorentz del plasmón eyectado excede al del jet normal, ya que libera mayor cantidad de energía potencial. Esto se interpreta en relación con la llegada del radio interno del disco hasta regiones más próximas al agujero negro (ver panel inferior en la figura). El choque interno de la nube rápida $(\Gamma>2)$ con el material más lento del jet original probablemente distorciona todo el jet. Se produce en el disco la transición hacia el estado dominado por emisión térmica y ya no se observa emisión proveniente de la base del jet, sino tal vez la emisión más retirada de los plasmones que se alejan, en direcciones opuestas, a gran velocidad 13 .

El destello en radio asociado con la transición podría coincidir con el momento mismo en el cual el material de la corona es acelerado y en última instancia evacuado. Esta idea de la pérdida del material más próximo al objeto compacto coincide con lo que sugiere el análisis de la Figura 4.5, comentado al inicio de esta sección.

En este escenario de "choque interno" permanece sin explicación qué es exactamente lo que desencadena la transición en las propiedades del espectro en radio. Resultados recientes parecen cuestionar algunas de las premisas sobre las que se basa este esquema (Gallo, 2007 y referencias). El refinar este modelo unificador es un desafío tanto teórico como observacional.

\footnotetext{
${ }^{12}$ Desafortunadamente, esto es díficil de relacionar con cantidades físicas, y la dureza depende de las bandas elegidas.

${ }^{13}$ Una disminución de velocidad del material eyectado se ha observado en los jets de los microcuásares XTE J1748-288 y XTE J1550-564 (Hjellming et al. 1999, Corbel et al. 2002).
} 


\subsection{Microcuásares en la Galaxia}

A lo largo de este capítulo se introdujo el concepto de MQ, y los rasgos principales de esta clase de objetos. El panorama que se presentó es el resultado de la elaboración de la información disponible e intentos por modelarla, sobre los $\sim 16$ microcuásares conocidos en la Galaxia cuya lista presentamos a continuación. A pesar de la brevedad de esta lista, la población de MQs es probablemente mucho mayor, abarcando talvez un $70 \%$ de las binarias de rayos X (Fender \& Maccarone, 2004).

Notese que en algunos MQs el movimiento aparente de los jets, proyectado en el cielo, es "supralumínico" $\beta_{\text {apar }}=v_{\text {apar }} / c>1$, donde $c$ es la velocidad de la luz (ej. Mirabel et al. 1994). Este es un efecto de aberración relativista y ocurre cuando la fuente se mueve en una dirección próxima a la de línea de la visua 14 con velocidad comparable a la de la luz (Blandford, McKee \& Rees, 1977). Cuando vemos directamente dentro del jet de un MQ o en una dirección muy próxima, este suele decirse que es un microblazar.

En algunos casos el torque gravitacional de la estrella donante de masa, que se encuentra en una órbita no coplanar con el disco de acreción, puede inducir la precesión de los jets

(ej. Kaufman Bernadó et al. 2002).

\footnotetext{
${ }^{14} \mathrm{Si} \theta$ es el ángulo entre la velocidad y la línea de la visual, entonces la velocidad transversal que infiere el observador es $\beta_{\text {apar }}=\frac{\beta \sin \theta}{1-\beta \cos \theta}$ donde $\beta$ es la velocidad relativa. Así $\beta_{\text {apar }}$ puede tomar valores arbitrariamente grandes a medida que $\beta \rightarrow 1$ y $\theta \rightarrow 0$.
} 
Tabla 4.1: Microcuásares de nuestra Galaxia, Paredes (2005) con ligeras modificaciones.

\begin{tabular}{|c|c|c|c|c|c|c|c|c|c|}
\hline Nombre & $\begin{array}{c}\text { Tipo de } \\
\text { sistema }^{(a)}\end{array}$ & $\begin{array}{c}D \\
(\mathrm{kpc})\end{array}$ & $\begin{array}{c}P_{\text {orb }} \\
\text { (d) }\end{array}$ & $\begin{array}{c}M_{\text {compacta }} \\
\quad\left(M_{\odot}\right)\end{array}$ & $\begin{array}{l}\text { Actividad } \\
\text { radio }^{(b)}\end{array}$ & $\beta_{\text {apar }}$ & $\theta^{(\mathrm{c})}$ & $\begin{array}{l}\text { Tamaño } \\
\text { jet (UA) }\end{array}$ & Comentario $^{(d)}$ \\
\hline \multicolumn{10}{|c|}{ Binarias de gran masa (HMXB) } \\
\hline LS I +61303 & $\begin{array}{l}\mathrm{B} 0 \mathrm{~V} \\
+\mathrm{NS} ?\end{array}$ & 2.0 & 26.5 & - & $\mathrm{p}$ & $\geq 0.4$ & - & $10-700$ & Prec? \\
\hline V4641 Sgr & $\begin{array}{l}\text { B9III } \\
+\mathrm{BH}\end{array}$ & $\sim 10$ & 2.8 & 9.6 & $\mathrm{t}$ & $\geq 9.5$ & - & - & \\
\hline LS 5039 & $\begin{array}{c}\mathrm{O} 6.5 \mathrm{~V}((\mathrm{f})) \\
+\mathrm{NS} ?\end{array}$ & 2.9 & 4.4 & $1-3$ & $\mathrm{p}$ & $\geq 0.15$ & $<81^{\circ}$ & $10-1000$ & Prec? \\
\hline SS 433 & $\begin{array}{c}\text { evolved A? } \\
\quad+\mathrm{BH} ?\end{array}$ & 4.8 & 13.1 & $11 \pm 5 ?$ & $\mathrm{p}$ & 0.26 & $79^{\circ}$ & $\sim 10^{4}-10^{6}$ & $\begin{array}{l}\text { Prec } \\
\text { XRJ }\end{array}$ \\
\hline Cygnus X-1 & $\begin{array}{c}\text { O9.7Iab } \\
+\mathrm{BH}\end{array}$ & 2.5 & 5.6 & 10.1 & $\mathrm{p}$ & - & $40^{\circ}$ & $\sim 40$ & \\
\hline Cygnus X-3 & $\begin{array}{l}\text { WNe } \\
+\mathrm{BH} ?\end{array}$ & 9 & 0.2 & - & $\mathrm{p}$ & 0.69 & $73^{\circ}$ & $\sim 10^{4}$ & \\
\hline
\end{tabular}

Binarias de pequeña masa (LMXB)

\begin{tabular}{|c|c|c|c|c|c|c|c|c|c|}
\hline XTE J1118+480 & $\begin{array}{c}\mathrm{K} 7 \mathrm{~V}-\mathrm{M} 0 \mathrm{~V} \\
+\mathrm{BH}\end{array}$ & 1.9 & 0.17 & $6.9 \pm 0.9$ & $\mathrm{t}$ & - & - & $\leq 0.03$ & \\
\hline Circinus X-1 & $\begin{array}{c}\text { Subgiant } \\
+\mathrm{NS}\end{array}$ & 5.5 & 16.6 & - & $\mathrm{t}$ & $>15$ & $<6^{\circ}$ & $>10^{4}$ & \\
\hline XTE J1550-564 & $\begin{array}{c}\mathrm{G} 8-\mathrm{K} 5 \mathrm{~V} \\
\quad+\mathrm{BH}\end{array}$ & 5.3 & 1.5 & 9.4 & $\mathrm{t}$ & $>2$ & - & $\sim 10^{5}$ & XRJ \\
\hline Scorpius X-1 & $\begin{array}{l}\text { Subgiant } \\
+\mathrm{NS}\end{array}$ & 2.8 & 0.8 & 1.4 & $\mathrm{p}$ & 0.68 & $44^{\circ}$ & $\sim 40$ & \\
\hline GRO J1655-40 & $\begin{array}{l}\text { F5IV } \\
+\mathrm{BH}\end{array}$ & 3.2 & 2.6 & 7.02 & $\mathrm{t}$ & 1.1 & $72^{\circ}-85^{\circ}$ & 8000 & Prec? \\
\hline GX 339-4 & $\begin{array}{c}- \\
+\mathrm{BH}\end{array}$ & $>6$ & 1.76 & $5.8 \pm 0.5$ & $\mathrm{t}$ & - & - & $\sim 10^{4}$ & \\
\hline 1E $1740.7-2942$ & $\begin{array}{c}- \\
+\mathrm{BH}\end{array}$ & $8.5 ?$ & $12.5 ?$ & - & $\mathrm{p}$ & - & - & $\sim 10^{6}$ & \\
\hline XTE J1748-288 & $\begin{array}{c}- \\
+\mathrm{BH} ?\end{array}$ & $\geq 8$ & $?$ & $>4.5 ?$ & $\mathrm{t}$ & 1.3 & - & $>10^{4}$ & \\
\hline GRS $1758-258$ & $\begin{array}{c}- \\
+\mathrm{BH} ?\end{array}$ & $8.5 ?$ & $18.5 ?$ & - & $\mathrm{p}$ & - & - & $\sim 10^{6}$ & \\
\hline GRS $1915+105$ & $\begin{array}{c}\mathrm{K}-\mathrm{M} \text { III } \\
+\mathrm{BH}\end{array}$ & 12.5 & 33.5 & $14 \pm 4$ & $\mathrm{t}$ & \multicolumn{3}{|c|}{$1.2-1.766^{\circ}-70^{\circ} \sim 10-10^{4}$} & Prec? \\
\hline
\end{tabular}

Notas: ${ }^{(a)}$ NS: neutron star BH: black hole. ${ }^{(\mathrm{b})}$ p: persistente; t:transitoria. ${ }^{(\mathrm{c})}$ inclinación del jet. (d) Prec: precession; XRJ: X-ray jet.

No se han resuelto jets en XTE J1118+480, pero se infiere su presencia de la pendiente del espectro. 


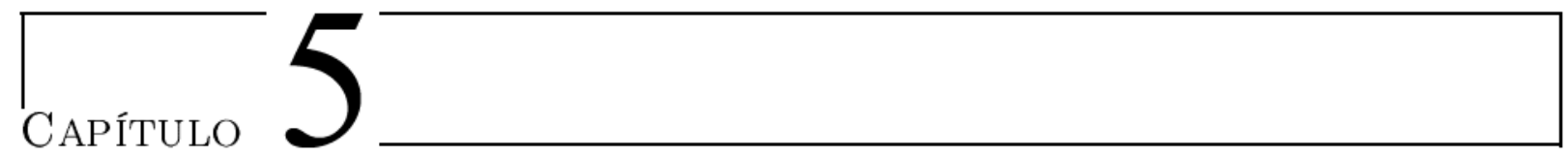

\section{Modelos para la emisión $\gamma$ de microcuásares}

Los microcuásares poseen todos los elementos para la potencial producción de rayos $\gamma$ : los jets conteniendo partículas relativistas con una velocidad de conjunto que es una fracción a veces importante de $c$, campos de fotones densos que pueden ser dispersados por electrones o positrones de los jets, y también (en el caso de MQs de gran masa), campos de materia en forma de viento estelar que pueden brindar blancos para interacciones hadrónicas. En la Figura 5.1 presentamos un esquema donde se muestran las diferentes componentes de un microcuásar y el rango del espectro electromagnético donde emiten.

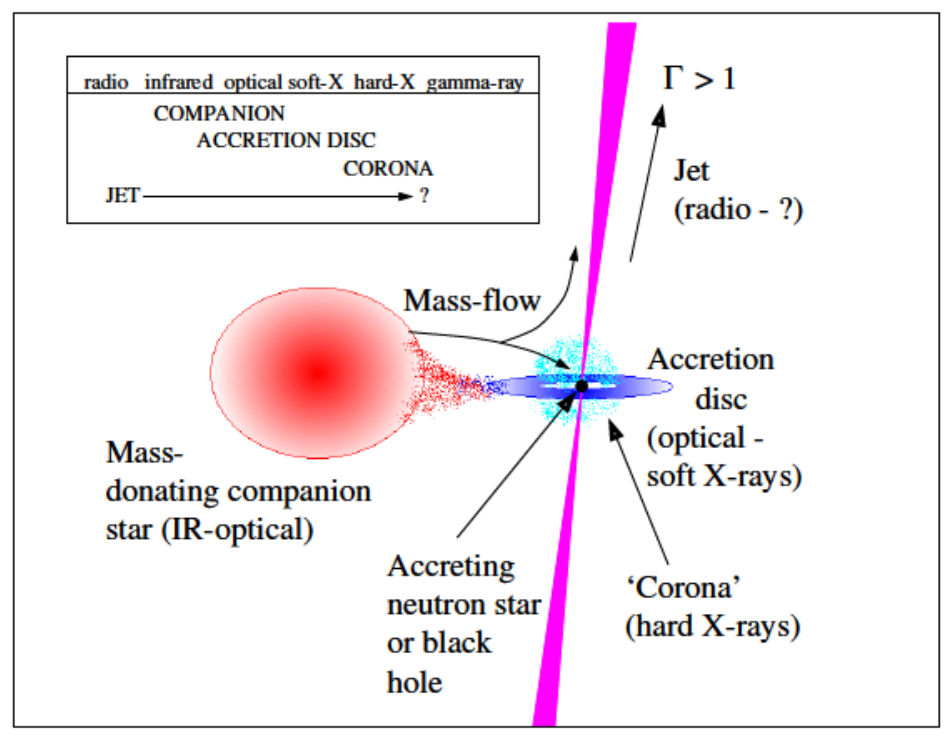

Figura 5.1: Principales componentes físicas de un MQ, esquema por Fender \& Maccarone (2004).

Habiéndose detectado a muy altas energías otros sistemas con elementos similares, como los blazares, en su momento resultó natural la proposición de los MQs como posibles 
contrapartidas para algunas de las fuentes no identificadas del catálogo EGRET. En particular, el interés por los MQs como potenciales fuentes de rayos $\gamma$ se intensificó a partir del descubrimiento de LS 5039, y la propuesta de Paredes et al. (2000) que asociaba este sistema con la fuente 3EG J1824-1514, sugiriendo que la emisión gamma podía originarse en la dispersión Compton inversa (mantendremos IC, por su sigla en inglés) de fotones provenientes de la compañera estelar. Trabajos subsecuentes por diversos autores han profundizado en la investigación de este escenario, incluyendo otros campos de fotones blanco o "semilla", y modelos de jets más sofisticados. La referencia original proponiendo a los MQs como una población de fuentes EGRET es Kaufman Bernadó et al. (2002), luego se amplió la discusión por ej. en Romero et al. (2004) y Bosch-Ramon et al. (2005). Paredes (2005) brinda una lista de las detecciones de algunos MQs por INTEGRAL, BATSE y tal vez COMPTEL y EGRET. Como se comentó en el Capítulo 2, la confirmación de los MQs como emisores variables hasta energías incluso en el rango $\mathrm{TeV}$ se produjo en 2005, cuando HESS detectó a LS 5039, por lo que hoy en día los MQs son reconocidos como emisores a lo largo de practicamente todo el espectro. Se trata, además, de fuentes que abarcan regiones espaciales de diferentes escalas (radio fuentes compactas, radio jets ligeramente extendidos y a veces estructuras de interacción con el medio muy extensas). Además de radiación electromagnética, alguna fracción de los rayos cósmicos galácticos podría originarse en MQs (Heinz \& Sunyaev, 2002), así como también neutrinos (Levinson \& Waxman 2001, Distefano et al. 2002, Aharonian et al. 2006).

Bosch-Ramon (2007) brinda un artículo de revisión actualizado sobre el modelado teórico de la emisión de MQs, enumera las complejidades que se presentan, y que han llevado a planteos muy simplificados en un esfuerzo de avance gradual. Los modelos a los que nos referiremos intentan describir la emisión a altas energías por parte de los jets estables que se relacionan con binarias en el estado espectral low/hard. En los trabajos que se comentarán se asume que la potencia cinética asociada con el material de los jets $L_{\mathrm{k} \text {, jet }}$ proviene solamente de energía acretada $L_{\mathrm{ac}}$, esto es, se desprecian otro tipo de fuentes, como podría serlo la rotación del objeto compacto. La re-aceleración de partículas tiene lugar a lo largo del jet, debido a ondas de choque producidas por gradientes de velocidad en el material eyectado (Rees 1978, en el contexto de AGNs y Kaiser et al. 2000, en el de los MQs). Este procesd 1 afecta a las partículas más energéticas de una dada distribución (cola supra-térmica), al mismo tiempo que actúan mecanismos de emisión y colisión que les hacen perder energía o "enfriarse".

Aunque no se asuma un mecanismo en particular para el lanzamiento de los jets, convendrá seguir el criterio consensuado de un origen magnetohidrodinámico. Si el jet es impulsado a través de la conversión de energía magnética en energía cinética, parece probable que la intensidad del campo magnético, $B$, sea cercana a la que se obtiene mediante una condición de equipartición de energía, al menos en la base del jet. Al alejarse de allí a lo largo del jet, tanto este campo magnético $B$ como la densidad de materia seguirán una evolución adiabática (si se asume que las líneas de fuerza están congeladas en el plasma).

\footnotetext{
${ }^{1} \mathrm{El}$ proceso básico es la aceleración de partículas difusiva en un medio magnetizado donde se propagan frentes de choque (ej. artículo de revisión de Drury 1983). En esta tesis, ver Apéndice.
} 
Los esfuerzos teóricos por modelar/predecir la emisión $\gamma$ de MQs se han bifurcado en dos tipos de aproximaciones: aquellas que asumen el liderazgo de los procesos radiativos por una población de electrones/positrones relativistas en los jets (modelos leptónicos) y otras, específicas de sistemas de gran masa, donde son protones relativistas en interacción con protones fríos los que dominan la producción de los rayos gamma por encima del $\mathrm{GeV}$. Estos últimos son los llamados modelos hadrónicos. De ambas clases de modelos podemos aprender sobre la importancia de los distintos mecanismos radiativos bajo diferentes circunstancias e intentar comprender qué nivel de detalle físico es necesario considerar para explicar los datos observacionales. Es importante aclarar que los modelos hadrónicos y leptónicos no se excluyen, sino que incluso se complementan, cómo será evidente en el Capítulo 8 A continuación presentamos las líneas generales de cada familia de modelos. Esta tesis trata en mayor medida con los modelos de tipo hadrónico, por lo que la sección correspondiente introduce mayor detalle. Más adelante, se comparan en forma explícita los resultados para la emisión $\gamma$ de modelos leptónicos y hadrónicos. Baste aquí adelantar que no existen aún, desde el punto de vista teórico, motivos para rechazar ninguno de los dos modelos.

\subsection{Modelos leptónicos}

En este contexto, los procesos radiativos que ocurren a lo largo del jet producen emisión en todo el espectro. La emisión sincrotrón se extiende desde la banda radio hasta los rayos $\mathrm{X}$ (Markoff et al. 2003) o incluso, el rango gamma con energías de algunos GeVs, cuando se asume un campo magnético cercano al de equipartición. En este caso además, cerca de la base del jet, puede ser importante una componente de emisión sincrotrón auto Compton (Band \& Grindlay 1986, Aharonian \& Atoyan 1999). Dispersiones IC entre leptones del jet y los fotones provenientes de campos externos (de la estrella, disco de acreción o de la corona) dominan la emisión gamma a muy altas energías (Kaufman-Bernadó et al. 2002). En binarias de gran masa, esta componente puede extenderse hasta energías cercanas al $\mathrm{TeV}$ (Bosch-Ramon et al. 2006b, Dermer \& Böttcher 2006). Una componente menor resulta de la aceleración de los leptones en el campo de los núcleos (Bremsstrahlung relativista).

Modelos leptónicos para sistemas con gran y pequeña masa han sido presentados en Bosch-Ramon et al. (2006a) bajo la hipotesis de que los jets están dinámicamente dominados por protones fríos. El valor medio de la energía cinética de los protones fríos viene dado por $\left\langle E_{p}^{\text {kin }}\right\rangle=m_{p} v_{\exp }^{2} / 2$, donde la velocidad de expansión lateral $v_{\exp }$ es una fracción de la velocidad de propagación del jet que determina el ángulo de apertura del cono que este forma. A diferencia con tratamientos anteriores, en estos modelos se ha buscado respetar la conservación de la energía 2 , y obtener el factor de Lorentz de los jets en forma consistente.

\footnotetext{
${ }^{2}$ La energía del material acretado se distribuye de la siguiente manera: en parte es advectada hacia el objeto compacto, en parte brinda la potencia $L_{\mathrm{k}, \text { jet }}$ a los jets, y otra parte es radiada por el disco y la corona. A su vez, en $L_{\mathrm{k} \text {, jet }}$ se suman la energía necesaria para sacar al material del pozo de potencial con la energía cinética que adquiere.
} 


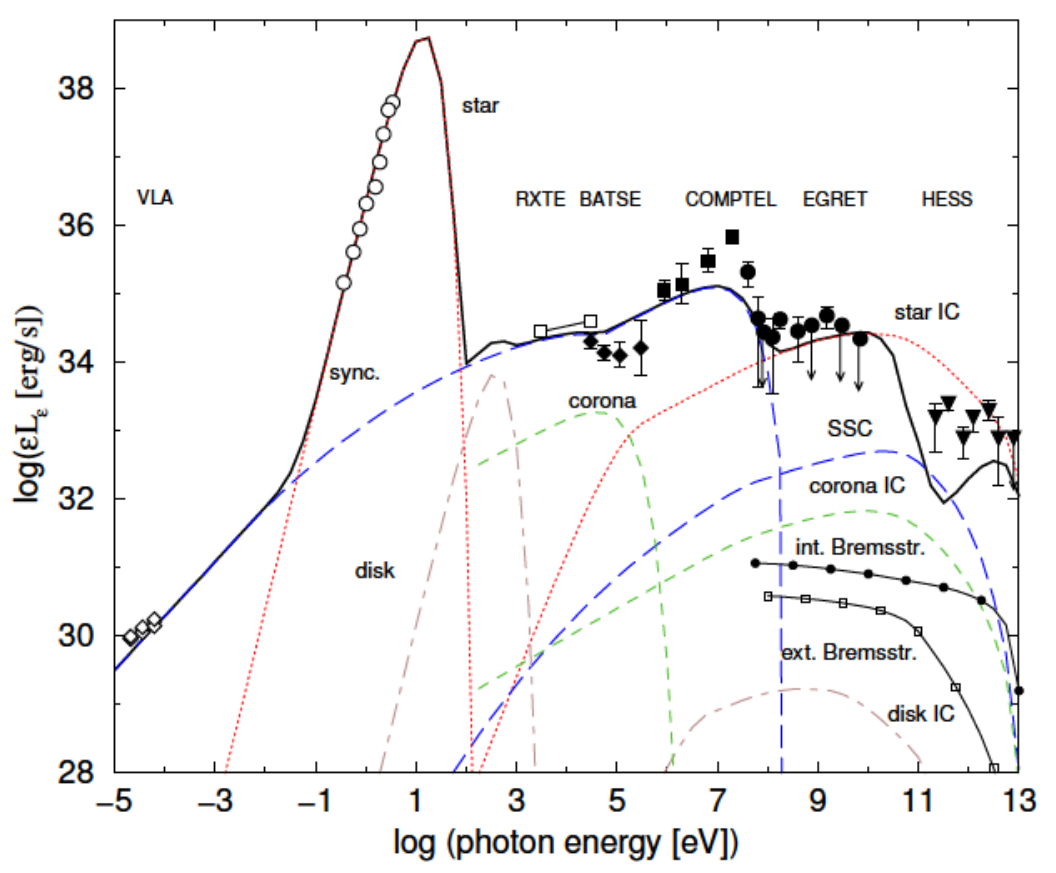

Figura 5.2: Distribución espectral de energía en un modelo leptónico para LS 5039, por Paredes et al. (2006). Se indica la emisión total en línea gruesa, y los valores observados por distintos instrumentos.

Respetando lo inferido de la emisión sincrotrón en radio, la distribución en energías de los leptones suele asumirse como una ley de potencias $n\left(z, E_{e}\right)=K_{e}(z) E_{e}^{-p}$ a la cual se impone un corte exponencial $\sim e^{-E_{e} / E_{e}^{\max }}$. El valor de la energía máxima de los leptones $E_{e}^{\max }$ se obtiene de la condición de balance entre la tasa de ganancia de energía (por aceleración difusiva de los electrones y su correspondiente tasa de pérdida de energía, a la cual pueden contribuir todos los procesos radiativos antes mencionados).

En la Figura 5.2 se muestra la distribución espectral de energías de un modelo leptónico para LS 5039, atenuada por la absorción de fotones. Este último efecto será tratado más adelante. Tanto aquí como en modelos hadrónicos, el conjunto de parámetros libres incluye algunos valores que no están bien acotados aún (por ej, factores de Lorentz mínimos de las partículas, eficiencias de aceleración difusiva, etc), para los que se adoptan valores típicos, que se espera sean estimaciones razonables desde un punto de vista físico, bajo ciertas condiciones.

\subsection{Modelos hadrónicos}

En microcuásares de gran masa se dará inevitablemente una interacción entre el viento estelar y los jets, que puede resultar en la producción de rayos $\gamma$ de fotones con muy 
alta energía. Interacciones de tipo hadrónico con concentraciones (nubes) de material del viento fueron analizadas como posibles fuentes transitorias de rayos $\gamma$ por Aharonian \& Atoyan (1996), para el caso de sistemas binarios con pulsares. Más adelante, Romero et al. (2003) desarrollaron el modelo hadrónico para MQs como fuentes de emisión $\gamma$ persistente. Recientemenet, este modelo ha sido adaptado al caso de MQs donde el viento presenta estructura de clumps (Romero et al. 2007b).

La idea básica del modelo es que el mismo mecanismo que acelera leptones opera sobre protones y núcleos, de manera tal que una fracción de la potencia del jet se manifiesta en forma de hadrones relativistas. Los protones del viento estelar se difunden dentro de los jets, y como resultado de interacciones $p p$ inelásticas con protones relativistas del jet 3 , se crean piones neutros que luego decaen emitiendo fotones gamma $\pi^{0} \rightarrow \gamma+\gamma$. En este modelo original de Romero et al. (2003) se adopta un viento estelar con simetría esférica y una órbita circular para el sistema binario. Por simplicidad, se asume que los jets son perpendiculares al plano orbital. En la Figura 5.3 se muestra un esquema de la situación discutida y las distribuciones de energía calculadas. Para determinar el contenido material de los jets, se adopta la hipótesis de acoplamiento propuesta por Falcke \& Biermann (1995) y aplicada con éxito en AGNs; la potencia cinética total del jet $Q_{j}$ es una fracción de la potencia asociada con la tasa de acreción, para lo cual se introduce el parámetro de acoplamiento $q_{j} \sim 10^{-3}-10^{-1}$ de modo que

$$
Q_{j}=q_{j} \dot{M}_{\mathrm{acc}} c^{2}
$$

donde $\dot{M}_{\text {acc }}$ es la tasa de acreción de masa sobre el objeto compacto. El valor supuesto por Romero et al. (2003) en su modeld $10^{-8} \mathrm{M}_{\odot} \mathrm{yr}^{-1}$, es similar a la de un sistema como Cygnus X-1.

\subsubsection{La distribución de protones relativistas}

Como resultado de procesos de aceleración difusiva, la distribución en energías de los protones relativistas, en el sistema de referencia có-movil al jet, sigue una ley de potencias $N_{p}^{\prime}\left(E_{p}^{\prime}\right)=K_{p} E_{p}^{\prime-\alpha}$, válida para $E_{p}^{\prime \min } \leq E_{p}^{\prime} \leq E_{p}^{\prime \max }$. El flujo correspondiente viene dado por

$$
J_{p}^{\prime}\left(E_{p}^{\prime}\right)=(c / 4 \pi) N_{p}^{\prime}\left(E_{p}^{\prime}\right)
$$

En la dirección $z$, perpendicular al disco de acreción, se orienta el eje del jet que tiene una geometría cónica, con radio $R_{\mathrm{j}}(z)=z\left(R_{0} / z_{0}\right)$. Aquí $z_{0}$ es la distancia desde el objeto compacto al sitio donde se piensa que el jet se ha formado. Típicamente, $z_{0}$ mide unos centenares de $\mathrm{km}$, donde termina la corona. $R_{0}$ es el valor del radio del jet en la base. La

\footnotetext{
${ }^{3}$ En el Apéndice se recopilan los principales rasgos de las interacciones hadrónicas.

${ }^{4} \mathrm{Al}$ mismo tiempo, al haber asumido $M_{\mathrm{bh}}=10 \mathrm{M}_{\odot}$, vale la pena notar que $\dot{M}_{\mathrm{acc}} c^{2} \sim 0.5 L_{\mathrm{Edd}}$, y en parte por esto las luminosidades obtenidas en este trabajo son altas.
} 

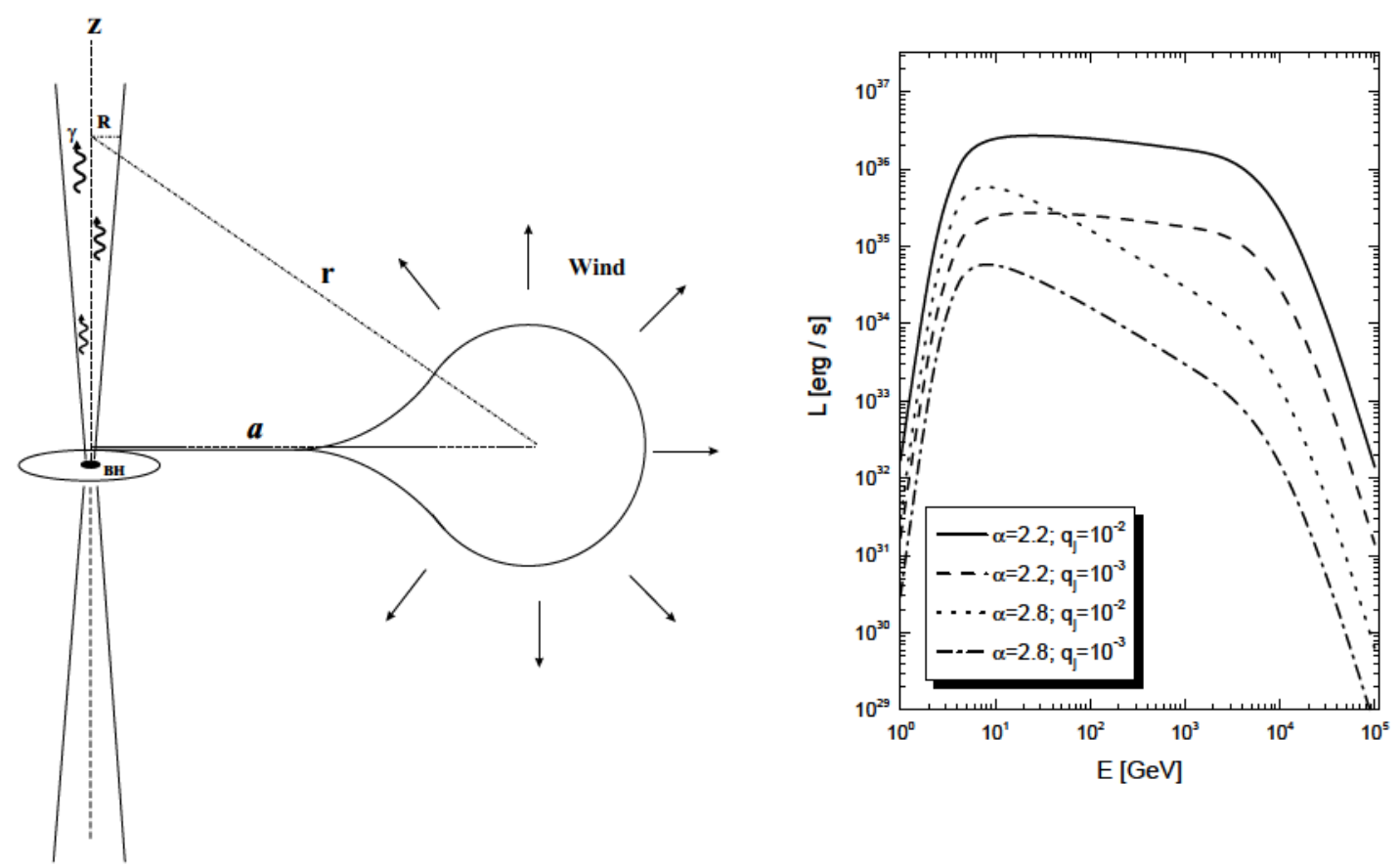

Figura 5.3: A la izquierda se muestra un esquema de la situación considerada en el modelo hadrónico de Romero et al. (2003). A la derecha, la distribución espectral de energía obtenida asumiendo distintos valores del índice espectral $\alpha$ y del parámetro de acoplamiento $q_{j}$. El ángulo de inclinación del sistema se asume de $10^{\circ}$.

expansión lateral del flujo de protones lleva a

$$
J_{p}^{\prime}\left(E_{p}^{\prime}, z\right)=\frac{c}{4 \pi} K_{0}\left(\frac{z_{0}}{z}\right)^{2} E_{p}^{\prime-\alpha}
$$

donde esta implícita la hipotesis de conservación del número de partículas (ver Ghisellini et al. 1985) y conservamos la tilde para cantidades medidas en el sistema del jet. Mediante el uso de invariantes relativistas puede probarse que en el sistema de referencia del observador (o laboratorio) la distribución anterior se transforma en

$$
J_{p}\left(E_{p}, \theta\right)=\frac{c K_{0}}{4 \pi}\left(\frac{z_{0}}{z}\right)^{2} \frac{\Gamma^{-\alpha+1}\left(E_{p}-\beta_{\mathrm{b}} \sqrt{E_{p}^{2}-m_{p}^{2} c^{4}} \cos \theta\right)^{-\alpha}}{\sqrt{\sin ^{2} \theta+\Gamma^{2}\left(\cos \theta-\frac{\beta_{\mathrm{b}} E_{p}}{\sqrt{E_{p}^{2}-m_{p}^{2} c^{4}}}\right)^{2}}},
$$

deducida en Purmohammad \& Samimi (2001). Aquí $\Gamma$ es el factor de Lorentz macroscópico del jet, con $\beta_{\mathrm{b}}$ la correspondiente velocidad en unidades de $c$, y $\theta$ es el ángulo subtendido entre la dirección de movimiento del proton y el eje $z$. 
La densidad numérica de partículas $n_{0}^{\prime}$ ingresando por la base del jet en $z_{0}$ cumple dos condiciones:

$$
n_{0}^{\prime}=\int_{E_{p}^{\prime \min }}^{E_{p}^{\prime \max }} N_{p}^{\prime}\left(E_{p}^{\prime}, z_{0}\right) d E_{p}^{\prime}
$$

y, por otro lado

$$
c \pi R_{0}^{2} n_{0}^{\prime}=\frac{Q_{\mathrm{j}}}{m_{p} c^{2}}
$$

donde $m_{p}$ es la masa en reposo del proton. Bajo la condición $E_{p}^{\prime \max } \ll E_{p}^{\prime \min }$ se puede despejar $K_{0}=n_{0}^{\prime}(\alpha-1)\left(E_{p}^{\prime \min }\right)^{\alpha-1}$, que es la constante de normalización en (5.4).

La energía máxima de una población de partículas se obtiene de la condición de balance entre las tasas de pérdida y ganancia de energía que las partículas sufren en el medio donde se encuentran. En el jet de un MQ, la ganancia de energía viene dada por la reaceleración en los frentes de choque, y depende de la intensidad del campo magnético turbulento, entre otros factores (ver Apéndice). En el caso general, los protones sufren pérdidas por foto-producción de piones $\left(p+\gamma \rightarrow \pi^{0}+p\right.$ y $p+\gamma \rightarrow \pi^{+}+n$ o producciones múltiples a energías muy altas) y por la creación de pares cuando fotones interactúan con su campo Coulombiano. Mannheim \& Schlickeiser (1992) brindan expresiones para las correspondientes tasas $d E / d t$. La emisión sincrotrón de protones suele ser un proceso ineficiente de enfriamiento, a menos que el campo magnético sea muy intenso. La energía máxima de los protones relativistas en el jet suele estar más fuertemente acotada por el tamaño de la región de aceleración que por las pérdidas energéticas, ya que el radio de giro, $r_{g}$, del protón se mantiene menor que el radio del jet. Igualando estas distancias se tiene

$$
r_{g}=\frac{E_{p}^{\max }}{e B(z)}=R_{j}(z)
$$

por lo que la energía máxima puede ser función de la altura $z$.

\subsubsection{Cálculo de la emisión gamma hadrónica}

La interacción de protones dá como resultado la producción de piones a través de las cadenas $p+p \rightarrow \xi_{\pi^{0}} \pi^{0}+\xi_{\pi^{-}} \pi^{-}+\xi_{\pi^{+}} \pi^{+}$, donde $\xi$ es la correspondiente multiplicidad. El decaimiento de los piones neutros lleva a la producción de rayos $\gamma$. La importancia de este proceso para la astronomía de altas energías fue enfatizada por pioneros del área como Ginzburg \& Syrovatskii (1964). La emisividad que depende del flujo incidente $J_{p}$, y como $\pi^{0} \rightarrow 2 \gamma$ es un decaimiento con probabilidad isotrópica en el sistema propio del pión, se tiene

$$
q_{\gamma}\left(E_{\gamma}\right)=2 \int_{E_{\pi}^{\min }}^{\infty} \frac{q_{\pi^{0}}\left(E_{\pi}\right)}{\sqrt{E_{\pi}^{2}-m_{\pi}^{2} c^{4}}} d E_{\pi}
$$

donde

$$
E_{\pi}^{\min }\left(E_{\gamma}\right)=E_{\gamma}+\frac{m_{\pi}^{2} c^{4}}{4 E_{\gamma}}
$$


La tasa de producción, o emisividad de piones es

$$
q_{\pi^{0}}\left(E_{\pi}\right)=\int_{E_{p}^{\mathrm{th}}\left(E_{\pi^{0}}\right)}^{E_{p}^{\max }} J_{p}\left(E_{p}\right) \frac{d \sigma_{p p}\left(E_{\pi^{0}}, E_{p}\right)}{d E_{\pi^{0}}} d E_{p},
$$

donde $E_{p}^{\text {th }}$ es la energía umbral para que ocurra la interacción generando un pión neutro con energía $E_{\pi^{0}} 6$ y se obtienen distintas aproximaciones según la expresión que se utilice para la sección eficaz diferencial (ver Apéndice). El formalismo de la aproximación $\delta$ propuesto por Aharonian \& Atoyan $(1996,2000)$ puede aplicarse en la mayoría de los casos.

La intensidad específica (fotones por unidad de tiempo, energía y ángulo sólido) de los rayos $\gamma$ resulta

$$
I_{\gamma}\left(E_{\gamma}\right)=\int n(r) q_{\gamma}\left(E_{\gamma}\right) d V
$$

donde $V$ es el volumen de interacción y $n(r)$ la densidad de los protones blanco (en reposo). Finalmente $L\left(E_{\gamma}\right)=4 \pi E_{\gamma}^{2} I_{\gamma}\left(E_{\gamma}\right)$ es la luminosidad (isotrópica). La dependencia exponencial de la sección eficaz $p p$ con la componente transversal del impulso del protón incidente lleva a que la emisión de los fotones resulte confinada en un ángulo pequeño $(\sim 0.17 / \Gamma)$ respecto de la dirección del protón, justificando que se asuma $\theta \approx \theta_{\text {obs. }}$. Así, para un flujo como (5.4) la emisión resulta fuertemente anisotrópica.

Antes de concentrarnos en los avances que esta tesis ha aportado aplicando el modelo hadrónico, se introduce en el capítulo siguiente, el proceso de absorción que puede afectar a los fotones gamma de muy alta energía, independientemente de cuál sea el mecanismo de emisión.

\footnotetext{
${ }^{5}$ Aquí $q_{\pi}$ expresa la cantidad de piones por átomo, por unidad de energía, ángulo sólido y tiempo. A veces se define $q_{\pi}$ incorporando el producto con la densidad $n$ de protones blanco.

${ }^{6}$ En la integral, debe reemplazarse por la energía mínima de los protones, en el caso de ser ésta mayor que $E_{p}^{\text {th }}$.
} 


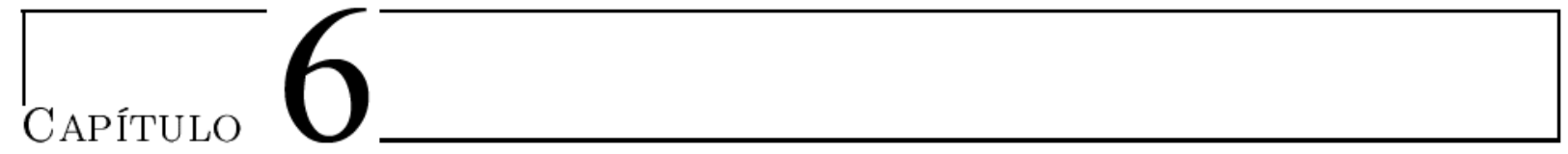

\section{Propagación de los rayos $\gamma$}

Una vez que los fotones $\gamma$ son creados en una fuente astrofísica por partículas relativistas, pueden ser absorbidos por campos magnéticos, de radiación o materia, presentes en la fuente misma o en su trayecto al observador. Cada uno de los mecanismos básicos de producción leptónica de rayos $\gamma$ (Bremsstrahlung relativista, dispersión Compton inversa y sincrotrón o radiación de curvatura, si el campo magnético es muy intenso) tiene como contrapartida un mecanismo de absorción con el mismo origen electromagnético y sección eficaz similar. El proceso que opera queda determinado por las características del medio donde se propagan los fotones. Es usual definir la profundidad óptica $\tau$ de un cierto "camino" que recorren los fotones de modo que si $I_{0}\left(E_{\gamma}\right)$ es la intensidad inicial emitida, luego de atravesar un medio con profundidad óptica $\tau$ la intensidad que resultante es

$$
I\left(E_{\gamma}\right)=I_{0}\left(E_{\gamma}\right) e^{-\tau} .
$$

En binarias masivas de rayos $\gamma$, donde la producción de los fotones muy energéticos puede ubicarse dentro del volumen que ocupa el sistema binario, el denso campo radiativo estelar actúa como blanco para la absorción de fotones y la consecuente materialización de pares a través del proceso $\gamma+\gamma \rightarrow e^{+} e^{-}$(Gould \& Schréder, 1967). Una estimación de la profundidad óptica para fotones con energía $E_{\gamma}$ emitidos a una distancia $r$ de una estrella cuyo campo radiativo tiene densidad $n_{\text {ph }}$ puede obtenerse calculando

$$
\tau\left(r, E_{\gamma}\right)=\int_{E_{\min }\left(E_{\gamma}\right)}^{\infty} \int_{r}^{\infty} n_{\mathrm{ph}}\left(E_{\star}, r^{\prime}\right) \sigma_{\gamma \gamma}\left(E_{\star}, E_{\gamma}\right) d r^{\prime} d E_{\star},
$$

donde $E_{\star}$ es la energía de los fotones estelares que usualmente siguen un espectro de cuerpo negro y $E_{\min }=\left(m_{e} c^{2}\right)^{2} / E_{\gamma}$ es la energía umbral para la creación de pares. $\sigma_{\gamma \gamma}\left(E_{\star}, E_{\gamma}\right)$ es la sección eficaz total para la interacción fotón-fotón, cuyo máximo se dá cerca del valor de umbral, y vale $\sigma_{\gamma \gamma} \sim \sigma_{T} / 2$ donde $\sigma_{T}$ es la sección eficaz de Thomson. Para fotones con $E_{\gamma} \sim \mathrm{TeV}$ este máximo ocurre precisamente para $E_{\star} \sim \mathrm{eV} \sim k T_{\star}$. En la Figura 6.1 se muestra la superficie que forma la profundidad óptica $\tau\left(r, E_{\gamma}\right)$, considerando una estrella de tipo espectral temprano (gran masa), con $T_{\star}=4 \times 10^{4} \mathrm{~K}$ y $R_{\star}=15 \mathrm{R}_{\odot}$. 

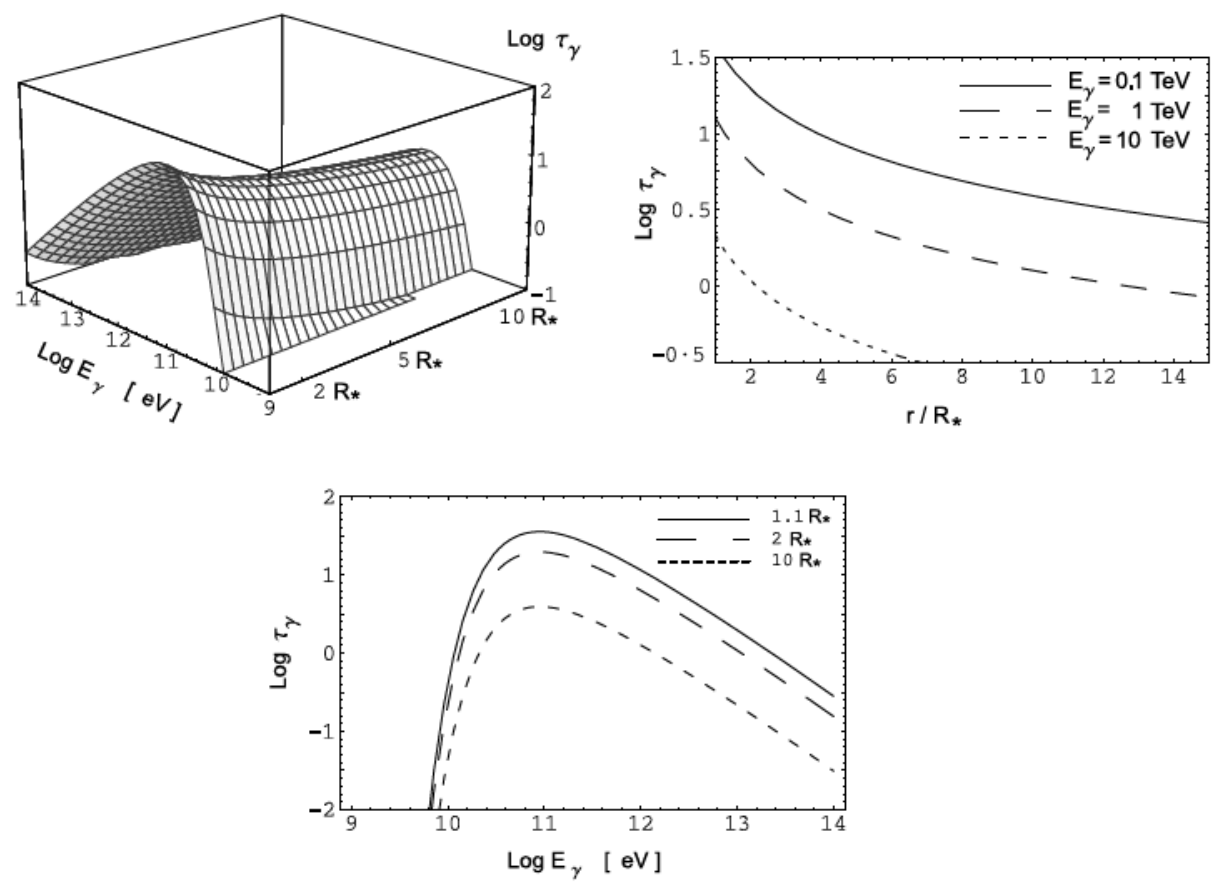

Figura 6.1: Profundidad óptica en función de la energía del fotón $\gamma$ y de la posición donde es emitido, utilizando la sección eficaz integrada en ángulos. La coordenada radial se aleja de la estrella, situada en el origen

Una expresión rigurosa para (6.2) incluye en forma explícita la dependencia con la geometría del sistema y de la interacción en si 1 , como han considerado por ej. Moskalenko (1995) y Dubus (2006c). El tamaño finito de la estrella se vuelve importante cuando se calcula la opacidad para fotones emitidos a distancia de algunos radios estelares de la estrella. Las fórmulas correspondientes se dan en el Apéndice. Conocidos los parámetros orbitales puede estimarse la variabilidad de la absorción y cómo esta ha de afectar a la emisión $\gamma$. La modulación inducida por la absorción puede proveer información sobre la fuente: su ausencia implica que la emisión gamma se produce en regiones más alejadas.

\subsection{Cascadas electromagnéticas}

En regiones en torno de objetos compactos galácticos y extragalácticos, por dar un ejemplo de fuentes confirmadas de rayos $\gamma$, donde ocurre la aceleración de partículas hasta muy altas energías, los procesos radiativos pueden actuar con mucha eficiencia y acompañados de las condiciones ideales para la absorción $(\tau>1)$, dando lugar a la creación de pares electrón - positrón que vuelven a emitir rayos $\gamma$. Las condiciones pueden ser propicias

\footnotetext{
${ }^{1}$ Ya que la sección eficaz queda parametrizada por $\beta=\left(1-\frac{2\left(m_{e} c^{2}\right)^{2}}{E_{\gamma} E_{\star}(1-\cos \theta)}\right)^{1 / 2}$, donde $\theta$ es el ángulo de interacción de los fotones.
} 
para que se desarrolle una cascada electromagnética mediante la cual la energía inicial del electrón (o fotón) se distribuye entre numerosas partículas y fotones secundarios. La Figura 6.2 muestra un esquema sumamente simplificado. La estructura del medio influirá sobre la cascada y así la absorción de fotones muy energéticos plantea un problema altamente complejo.

Una vez iniciada la cascada se desarrollará hasta que la escala temporal de los procesos radiativos que producen fotones fuera del rango $\gamma$ sea menor que la de los procesos que resultan en rayos $\gamma$. La cascada también se detendrá si el tiempo de enfriamiento radiativo de las partículas excede el tiempo característico de las pérdidas no-radiativas o si la opacidad a la propagación de los fotones $\gamma$ cae por debajo de 1.

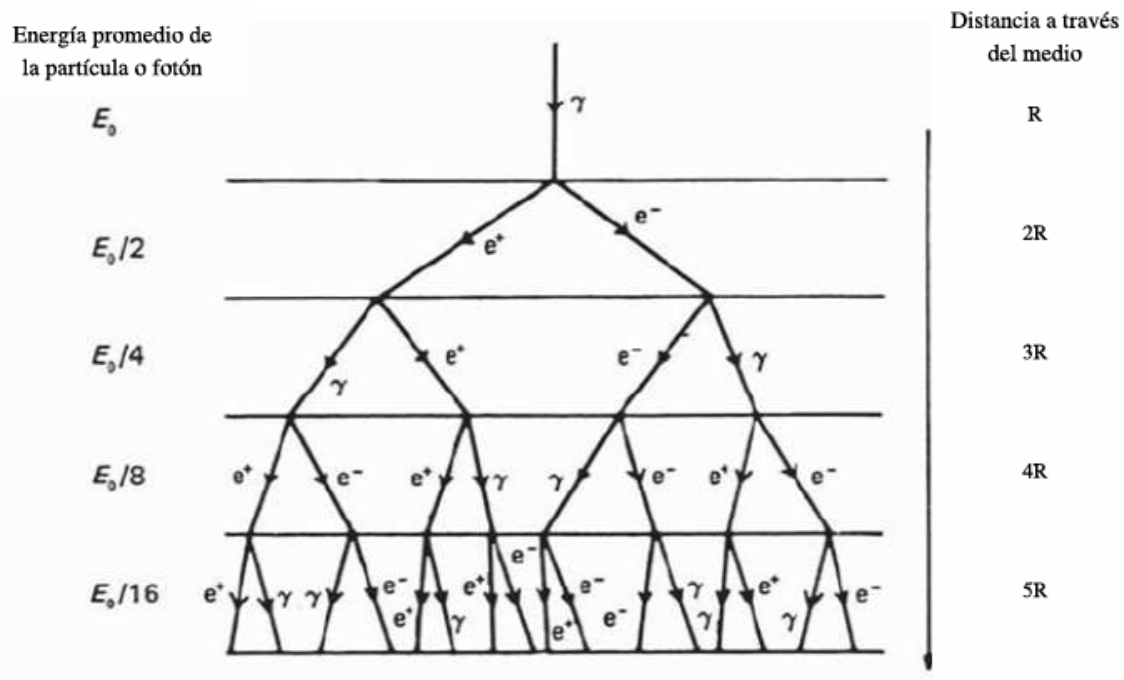

Figura 6.2: Esquema sencillo de una cascada electromagnética. En un límite en el que los caminos libres de fotones y electrones ultra-relativistas son similares $\sim R$, puede inferirse que el número total de partículas (leptones y fotones) aumenta exponencialmente con la distancia, mientras que la energía de cada una disminuye a la inversa. De Longair (1992).

Las características de cascadas electromagnéticas en medios materiales fueron originalmente estudiadas en el contexto de interacciones de rayos cósmicos con la atmósfera (mencionadas en la Sección 2.2). La teoría puede ser aplicada bajo las condiciones físicas que se dan en algunas fuentes de rayos $\gamma$, como por ejemplo en algunas binarias de rayos $\mathrm{X}$ donde se encuentre un gas denso iluminado por fotones $\gamma$ (ver Sección 10.1.1, donde se aplica la aproximación analítica obtenida por Rossi \& Greisen, 1941).

El desarrollo de cascadas en campos magnéticos es un elemento clave para el entendimiento de la física que opera en la magnetósfera de los pulsares, aunque este tipo de cascadas también ocurre en presencia de campos magnéticos más débiles 2 que en ese con-

\footnotetext{
${ }^{2}$ El parámetro relevante para que se desarrollen cascadas en campos magnéticos depende del producto entre la energía típica de las partículas involucradas y la intensidad del campo magnético (Anguelov \& Vankov, 1999 y sus referencias).
} 
texto, como en el campo magnético terrestre, y en algunos discos de acreción.

El desarrollo de cascadas electromagnéticas en un gas de fotones es un fenómeno común en la astrofísica, teniendo lugar en distintas escalas, involucrando desde objetos compactos como agujeros negros con acreción, eruptores de rayos $\gamma(\mathrm{GRBs})$, jets con dimensiones del orden del parsec en blazares, hasta las escalas de cientos de kpc que involucran la emisión de núcleos galácticos activos y Mpc en cúmulos de galaxias. Los rayos gamma de muy alta energía interactúan con los fotones de los fondos cósmicos (infrarrojo y de microondas) iniciando cascadas en todo el Universo (ej. Aharonian, 2004). La superposición de contribuciones de rayos $\gamma$ de estas cascadas constituye una fracción de la emisión difusa extragaláctica.

En principio, una cascada puede describirse por completo sólo si se tiene información sobre todas las partículas involucradas y sus interacciones en un amplio rango de energía. Desde un punto de vista teórico se busca obtener expresiones para las distribuciones de probabilidad de encontrar electrones y fotones en un cierto elemento de energía $d E$, y con coordenadas espaciales en $d \bar{r}$. Esto plantea un problema matemático formidable, por lo que se incorporan la mayor cantidad de simplificaciones posibles para obtener aproximaciones útiles, como lo han hecho Svensson (1987), Zdiarski (1988), Coppi \& Blandford (1990).

Es común considerar que la emisión producida por los electrones que participan de la cascada no (retro)alimenta nuevas interacciones con electrones, esto es, los $e^{ \pm}$sólo interactúan con fotones del medio, y se habla entonces de cascadas lineales. En ausencia de campos magnéticos, y a energías altas, los ángulos de emisión son lo suficientemente pequeños como para considerar que la cascada se desarrolla en la dirección de la partícula inicial. Se tratan entonces por separado la propagación longitudinal de la cascada y su esparcimiento lateral. El tratamiento unidimensional de cascadas parece ser más que suficiente en muchas situaciones astrofísicas. El comportamiento medio de estas cascadas puede ser descripto por un solo conjunto de ecuaciones integro-diferenciales, donde se reemplaza la sección eficaz diferencial según el medio considerado. Estas llamadas "ecuaciones adjuntas de la cascada" (Landau \& Rumer 1938) han sido resueltas en forma numérica por Aharonian \& Plyashesnikov (2003) para medios homogéneos (ver Sección A.5 del Apéndice). En este trabajo también se exploran cascadas que se desarrollan en medios que no son "puros", en donde puede cambiar el mecanismos que domina las pérdidas energéticas de los leptones. El impacto de tal interferencia se torna muy complejo, y es sensible a la elección de los parámetros principales, por lo que en cada caso es conveniente hacer un tratamiento particular.

En escenarios más realistas (relajando la condición de homogeneidad), las propiedades de cascadas lineales en campos radiativos han sido estudiadas usando simulaciones Monte Carlo (por ej. Bednarek 1997, 2000; Sierpowska \& Bednarek 2005). En estos trabajos se asume que la dirección de movimiento de los pares se isotropiza inmediatamente luego de su creación. La justificación para esta hipotesis es que la distancia de enfriamiento por dispersiones IC de los electrones es mayor que el radio de giro en la componente aleatoria del campo magnético asumido. 
Protheroe (1986) brinda una completa y clara descripción de un código programable para realizar las simulaciones de cascadas unidimensionales en un gas de fotones térmicos, con distribución isotrópica de los impulso 3 . El uso de técnicas Monte Carlo permite incorporar expresiones exactas de las secciones eficaces, en un tratamiento que tiene en cuenta los ángulos de interacción, aunque luego, por consideraciones de almacenamiento numérico, se guarda sólo la información sobre la propagación longitudinal de la cascada. Este artículo provee además de una descripción minuciosa de las reglas de muestreo para las funciones involucradas. En Protheroe et al. (1992) se describen las modificaciones hechas al programa original para tratar con un campo de fotones no homogéneo y anisotrópico. Basados en el esquema propuesto por estos dos trabajos, se desarrolló un nuevo código para los estudios implementados en esta tesis.

\section{2. $\quad$ El código desarrollado y algunas aplicaciones}

El programa que se desarrolló en lenguaje FORTRAN sigue en líneas generales el diagrama de flujo dado por Protheroe (1986) y que reproduce la Figura 6.3 A la simulación se ingresa la función que describe al espectro inyectado y la distancia $d_{\text {cascada }}$ entre la fuente y el observador, además de fijar el rango de energías que interesa. Se muestrea la energía de un fotón del espectro inyectado y se calcula su camino libre medio $\lambda$. Se muestrea un punto de interacción considerando una distribución exponencial $\propto e^{-x / \lambda}$, y se suma a la distancia que ya se haya recorrido. Si la distancia entre este punto y el observador es menor que $d_{\text {cascada }}$ se considera la interacción. A continuación se muestrea la energía de las dos partículas salientes: dos electrones cuando se crean pares (no se distinguen electrones de positrones) o un electrón y un fotón para las dispersiones IC. La energía y tipo de una de estas partículas es almacenado en una matriz de "reserva", junto con la distancia que ya se recorrió desde la fuente. Una nueva distancia de interacción se calcula para la otra partícula y se continúa procediendo como antes. Mientras la energías de las partículas está dentro del rango de interés, se detiene el avance de las iteraciones sólo cuando la distancia recorrida es mayor que $d_{\text {cascada }}$. Finalmente, se almacena a los fotones salientes para formar el espectro procesado por la cascada. Se retoma entonces una partícula de la reserva, hasta que se haya calculado la "descendencia" para todas las que hubiera. Cuando esto ocurre se vuelve a la instancia inicial, tantas veces como para muestrear en forma confiable el espectro inyectado (al menos varios miles de fotones).

Tanto en la simulación de la producción de pares como en la dispersión IC, antes que la interacción sea tratada, deben muestrearse una energía y ángulo de interacción correspondientes al fotón blanco, a partir de distribuciones apropiadas (que no son simplemente la

\footnotetext{
${ }^{3}$ Este código fue desarrollado para tratar con las cascadas IC que se desarrollan en el fondo cósmico de radiación (CMB).

${ }^{4}$ En nuestro trabajo, se utilizan las expresiones completas para $\sigma_{\gamma \gamma}$ (Gould \& Schréder, 1967) y $\sigma_{\gamma e}$ (Jauch \& Rohrlich 1976), parametrizadas en cada caso por el cuadrado de la energía total en el sistema centro de masa.
} 


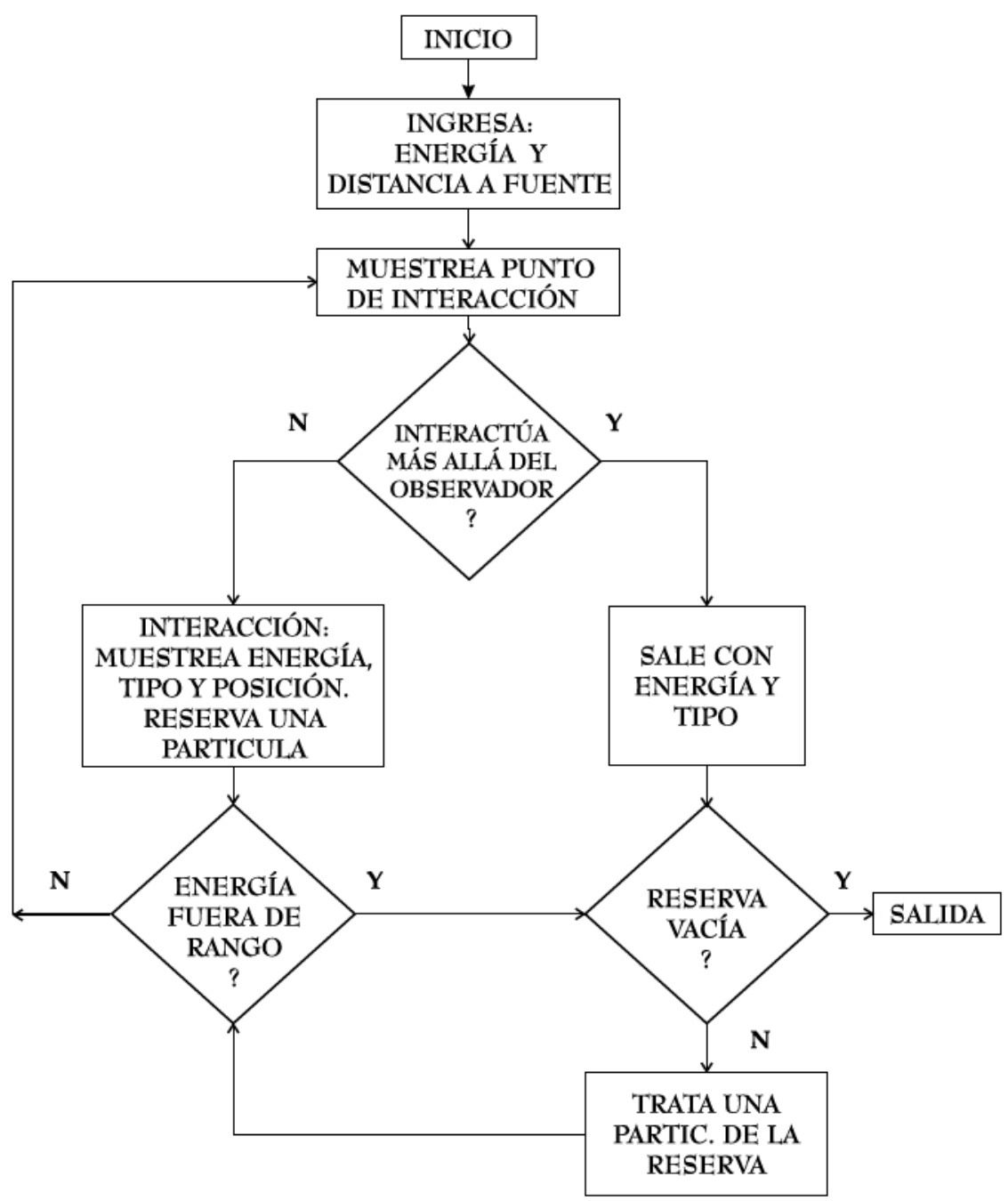

Figura 6.3: Diagrama de flujo del programa, originalmente por Protheroe (1986).

Planckiana e isotrópica) debidas a la velocidad relativa entre las partículas de la cascada y los fotones blanco, y a la dependencia de la sección eficaz con la energía total en el sistema centro de masas (CM). Con las energías y ángulos conocidos (ya muestreados) la creación de pares es tratada en el CM y luego se hace la transformación de Lorentz correspondiente, para pasar al sistema del observador. Para el tratamiento del scattering IC con fotones blanco que se distribuyen en forma isotrópica, expresiones detalladas para la distribución en energías de los fotones salientes han sido obtenidas por Blumenthal \& Gould (1970).

En la adaptación de este esquema que hemos hecho para simular cascadas que se desarrollan en el campo de fotones térmico de una estrella, se incorporó al cálculo de los caminos libres medios el tratamiento geométrico sugerido por Dubus (2006c) que se detalla en el Apéndice: hasta una distancia de unos cuantos radios estelares ( una decena) se tiene en cuenta el tamaño finito de la estrella. El camino libre de una partícula generada 
en la posición $\bar{r}$ respecto de la estrella depende entonces del ángulo $\psi$ que forma $\bar{r}$ con la línea de la visual. Establecida la posición donde ocurre una interacción, los fotones blanco provienen ahora solamente de la superficie estelar, la cual sustiene un cierto ángulo sólido de donde muestreamos la dirección del fotón blanco. Finalmente, para expresar el espectro de fotones $\gamma$ que emergen de la dispersión IC en el caso anisotrópico hemos seguido a Bednarek (1997, su Apéndice C) para adaptar la aproximación head-or 5 dada por Jones (1968).

Luego de una etapa de testeo, se implementó el código desarrollado en algunas aplicaciones concretas, variando la distancia a la estrella donde se produce la inyección de los rayos gamma $\left(d_{0}\right)$, el ángulo de observación $\left(\psi_{0}\right)$, y la pendiente del espectro inyectado (en principio, una ley de potencias de índice $\alpha$ ). Para justificar la aplicación del código, se calcularon antes las profundidades ópticas para los fotones $\gamma$ inyectados (esto es con $d_{0} \mathrm{y}$ $\left.\psi_{0}\right)$. Un hecho de características más generales es que por debajo de la condición umbral, la creación de pares se suprime en forma abrupta, mientras que a energías similares los electrones aún pueden interactuar 6 . Esto se ilustra en la Figura 6.4, donde se muestran las profundidades ópticas para fotones (y electrones), que se inyectan a distancia fija (aquí $d_{0}$ es la separación orbital, y se asume que los fotones $\gamma$ son generados cerca del objeto compacto) en un sistema de características similares a las de Cyg X-1. Se eligió como cero de la fase orbital al momento de conjunción inferior, cuando el punto de inyección se sitúa entre la estrella y el observador. La fase 0.5 es la conjunción superior, y fase 0.25 (ó 0.75 , con igual configuración) es un punto intermedio. En una órbita circular este ángulo de fase y la inclinación $(i)$ de la órbita son suficientes para determinar el ángulo $\psi_{0}$ que se usa como parámetro de la simulación (ver Apéndice).

Figura 6.4: Profundidad óptica para fotones y electrones bajo las siguientes condiciones iniciales.

\begin{tabular}{cr}
\hline Parámetro & valor \\
\hline$T_{\star}$ & $3 \times 10^{4} \mathrm{~K}$ \\
$R_{\star}$ & $15 \mathrm{R}_{\odot}$ \\
$d_{0}$ & $3 R_{\star}$ \\
$i$ & $30^{\circ}$ \\
$e$ & 0 \\
\hline
\end{tabular}

Orellana, Romero \& Pellizza (2006).

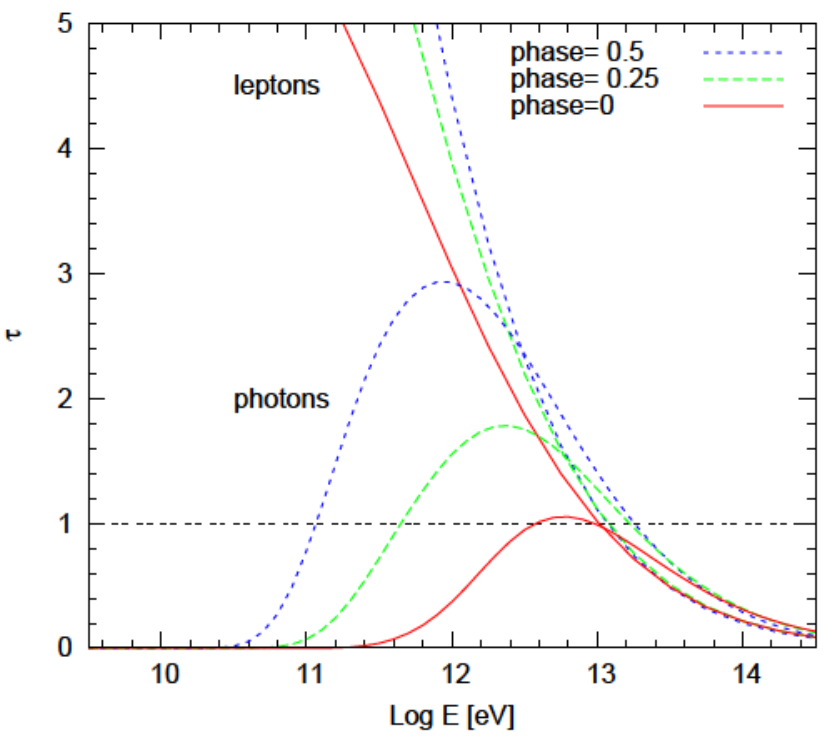

\footnotetext{
${ }^{5}$ En la aproximación head-on se considera que en el sistema de referencia del propio electrón, los fotones blanco se mueven en la dirección opuesta a la velocidad del electrón.

${ }^{6} \mathrm{~A}$ energías menores la sección eficaz aumenta al salir del regimen de Klein-Nishina.
} 
En la Figura 6.5 se muestran los espectros procesados por cascadas, para los mismos valores de fase orbital. El índice espectral de los fotones inyectados es $\alpha=1.94$, siendo $I(E) \propto E^{-\alpha}$, y el rango de energías es $10 \mathrm{GeV}$ a $10 \mathrm{TeV}$. La distancia hasta donde se ha seguido la evolución de las cascadas es $d_{\text {cascada }} \sim 10$ veces la separación orbital $d_{0}$. Los fotones que superan esta distancia son almacenados para generar el espectro procesado. A distancias mayores, la eficiencia de la cascada se reduce notablemente, debido a la disminución en la densidad de los fotones blanco. Los espectros fueron obtenidos mediante la elaboración de histogramas, en este caso, acomodando los fotones en intervalos de ancho $\Delta(\log E)=0.25$. Se impuso luego la conservación de la energía para obtener una normalización. En las curvas de los espectros procesados se reproduce la tendencia esperada de una mayor modificación del espectro original cuando mayor es el valor de $\tau_{\gamma \gamma}$ para las condiciones de inyección. En el rango de energías de algunos GeVs, el espectro se vé modificado a pesar de que $\tau_{\gamma \gamma} \ll 1$, debido justamente al procesamiento que realiza la cascada. Este es el efecto que más nos interesa estudiar. Para espectros inyectados más blandos que el de este ejemplo, digamos $\alpha>2$, no se espera que la cascada introduzca una modificación tan notoria, ya que la energía que se redistribuye proveniente del rango $\sim \mathrm{TeV}$ es desde el inicio menor que la que se encuentra en el rango $\sim \mathrm{GeV}$, donde no hay absorción.

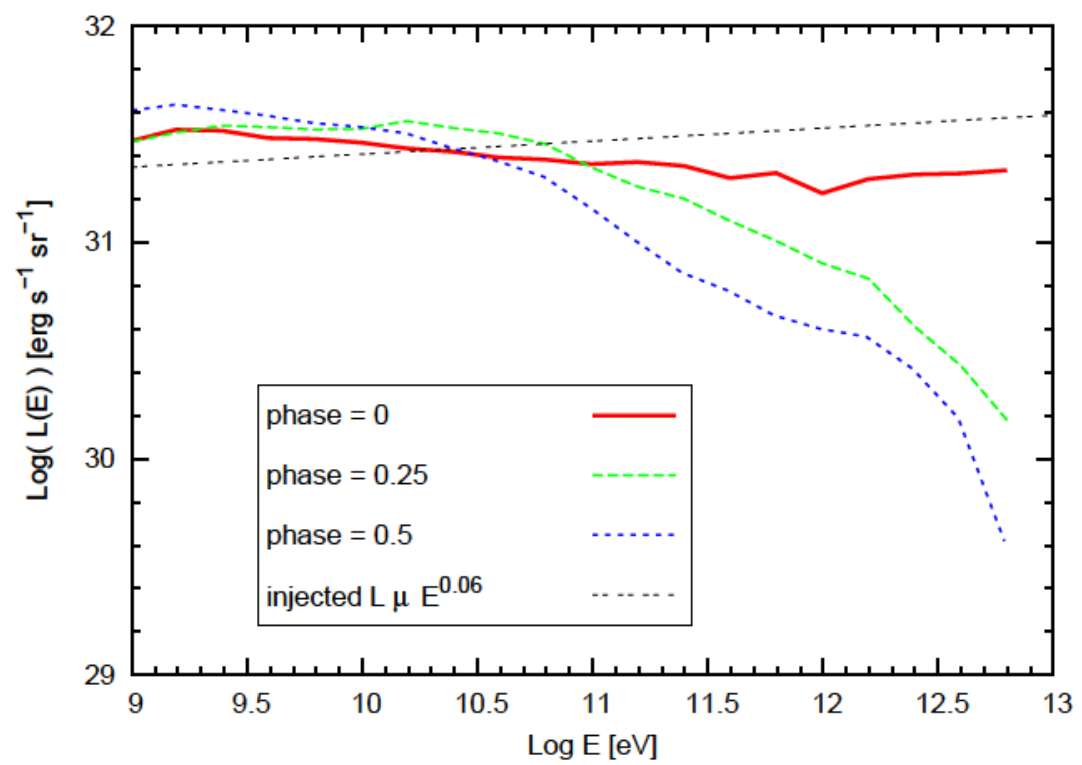

Figura 6.5: Espectros procesados por cascadas, calculados con el código desarrollado. Ver detalles en el texto. Se indica el espectro inyectado.

Las dispersiones IC son simuladas en detalle, de modo que si un electrón queda con energía suficiente (dentro del rango de interés) se lo continúa considerando. Es por esto que el número de interacciones (creación de pares más dispersiones IC) seguidas por el programa puede ser grande aún cuando la opacidad a la propagación de los fotones es tan baja como en el caso de fase orbital cero. A efectos ilustrativos, rotulamos las generaciones con el número de interacciones $\gamma \gamma$ que preceden a cada partícula, llamando generación 1 a 


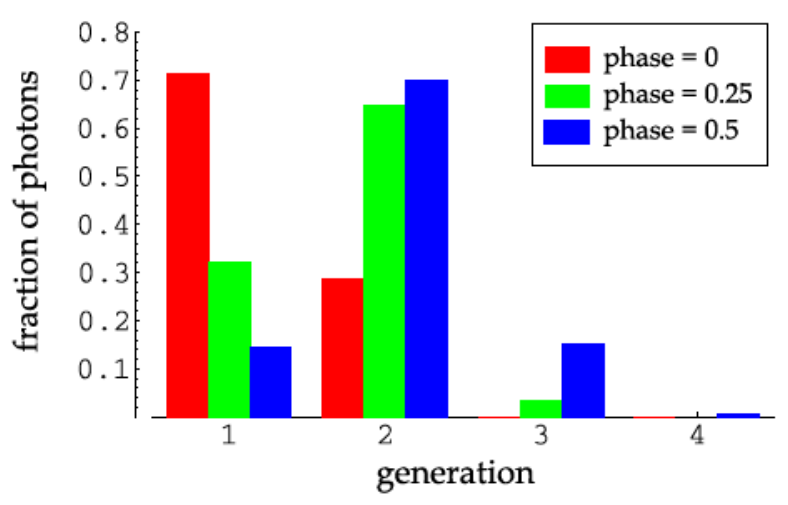

Figura 6.6: Número de generación de pares de los fotones que forman los espectros procesados de la Figura 6.5 Los fotones de la generación 1 son los originalmente inyectados que no fueron afectados por la absorción.

la de los fotones originalmente inyectados. La Figura 6.6 muestra el porcentaje de fotones procesados discriminados por generación. Por lo dicho, el número total de interacciones puede ser bastante mayor que el número de generación. Por ejemplo, algunos fotones han sufrido hasta 14 interacciones en el caso de fase 0.5 de esta aplicación. 
$\prod_{\text {CAPÍtUlo }}$

\section{Un modelo hadrónico para la emisión $\gamma$ del microcuásar LS I +61 303}

\subsection{Características de la fuente}

LS I +61 303 (del catálogo Luminous Stars of the Northern Milky Way I, Hardrop et al. 1959; también V615 Cas) es la contraparte óptica de la fuente variable en radio GT $0236+610$ (Gregory \& Taylor, 1978). Su emisión en rayos X se conoce ya desde comienzos de los años 1980 cuando fue detectada por el observatorio Einstein (Bignami et al. 1980). Se trata de un sistema binario formado por un objeto compacto y una estrella de tipo espectral B0 V con líneas de emisión anchas que revelan la presencia de material circunestelar (Hutching \& Crampton, 1981). Se ubica dentro de la caja de error de la fuente COS-B de rayos gamma 2CG 135 +01 (Hermsen et al. 1977), más tarde identificada con 3EG J0241+6103 (Hartman et al. 1999, Kniffen et al. 1997).

Observaciones en radio establecieron una modulación periódica (tipo flaring) de la fuente, con $P \simeq 26.5$ días (Taylor \& Gregory, 1982), que se interpreta como el período orbital del sistema. La emisión en el infrarrojo también varía con este período (Paredes, et al. 1994). El espectro en radio es no-térmico, y su grado de polarización lineal (Pecaraula et al. 1997) indica que se trata de emisión sincrotrón de electrones relativistas. Paredes (1987) encontró que el máximo de la intensidad en radio varía en una escala temporal de unos $\sim 4$ años. Gregory et al. (1989) propusieron que esto puede deberse a una variación cuasi-cíclica en las propiedades de la envoltura estelar de la estrella Be, o en forma alternativa, a la precesión de un jet (ver también Martí, 1993).

A través del modelado de la emisión IR 1 , Martí \& Paredes (1995) han caracterizado la envoltura circunestelar de la estrella Be, e inferido que la órbita del sistema tiene una

\footnotetext{
${ }^{1}$ Aplicando el método de la curva de crecimiento, y considerando atenuación por eclipses. Por "compañera" eclipsada, los autores aclaran que no se refieren a una estrella normal, sino que probablemente se eclipsa la emisión IR del material que está cayendo hacia el objeto compacto.
} 
excentricidad elevada. Por motivos históricos, es la fase en radio $(\phi)$ la que se utiliza para describir el movimiento orbital, siendo $\phi=\left(t-T_{o}\right) / P$ de modo que $0 \leq \phi<1$, siendo $t$ el tiempo y $T_{o}$ un momento de referencia 2 tal que el pasaje por el periastro ocurre en fase $\phi=0.23$. La emisión en radio se intensifica en $\phi \sim 0.45-0.9$.
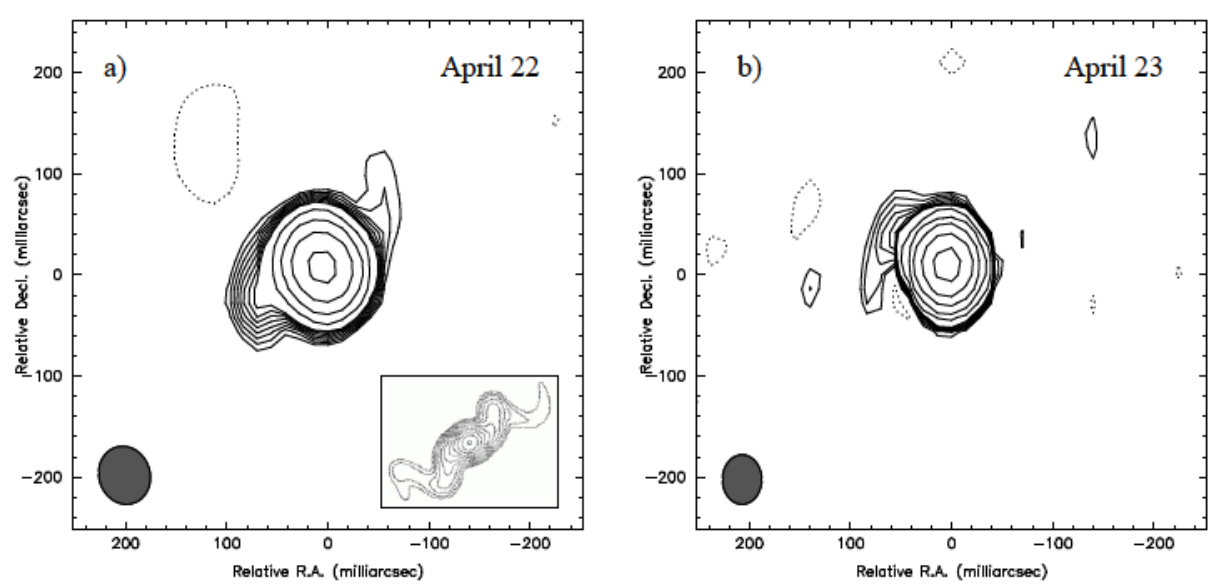

Figura 7.1: Mapas en radio ( $5 \mathrm{GHz})$ de LS I +61303 por Massi et al. (2004). Los autores enfatizan la similitud morfológica con el MQ con precesión SS433, cuyo mapa (simulado) muestran en el recuadro pequeño del panel a).

Massi et al. (2001) presentaron observaciones VLBI de LS I +61 303 donde se resuelve una estructura elongada, que puede interpretarse como un jet colimado y establece a LS I +61 303 como un MQ. Nuevas observaciones en radio (utilizando MERLIN) permitieron definir además un contrajet, de modo que la estructura se extiende unas $\sim 200 \mathrm{UA}$ a los lados de la fuente (Massi et al. 2004). La forma en S de los mapas (Figura 7.1) provee evidencia en favor de una precesión rápida (o inestabilidad direccional) de tales jets, induciendo variabilidad en una escala del orden del día. La velocidad macroscópica inferida es $\beta \sim 0.6$.

Paredes et al. (1997) analizaron datos de RXTE (2-10 keV), hallando actividad recurrente cuyo período coincide con el orbital. Otras observaciones en rayos X de LS I +61 303 incluyen datos de la mayoría de los intrumentos activos en los últimos 30 años: ROSAT, ASCA, Chandra, Beppo-SAX, XMM-Newton, INTEGRAL y Swift/XRT (ej. Taylor et al. 1996, Leahy et a. 1997, Greiner \& Rau 2001, Sidoli et al. 2006, Paredes et al. 2007, Chernyakova et al. 2006, Esposito et al. 2007). Asumiendo una distancia de $\sim 2 \mathrm{kpc}$ (Frail \& Hjellming 1991, y más tarde Steele et al. 1998), la luminosidad en la banda $0.3-10 \mathrm{keV}$ se estima que varía en $L_{X} \sim 3.4 \times 10^{33}-1.6 \times 10^{34} \mathrm{erg} \mathrm{s}^{-1}$. Estos valores son bajos en comparación con la luminosidad típica de una binaria de rayos $\mathrm{X} 3$. El índice espectral de los fotones $\mathrm{X}$ blandos es $\alpha \simeq 1.78 \pm 0.05$ (Esposito et al. 2007). Éstos siguen una ley de potencias sin componente de cuerpo negro, ni líneas. Tampoco se detectan pulsaciones, pero

\footnotetext{
${ }^{2}$ Explicitamente, $\phi=0$ en la fecha Juliana $T_{0}=2443366.775$, Taylor \& Gregory (1982).

${ }^{3}$ Además, esta potencia en rayos X está casi tres ordenes de magnitud por debajo de la luminosidad de Eddington, incluso para una estrella de neutrones.
} 
se han registrado variaciones en el flujo (flickering) de hasta un factor $\sim 3$ en lapsos temporales del orden de la hora, superpuestos a la modulación orbitalt Los datos más recientes indican que para rayos $\mathrm{X}$ de $1-100 \mathrm{keV}$, el flujo es máximo cerca de la fase $\phi \sim 0.55-0.65$. No se detectó emisión X durante el pasaje por el periastro, donde se espera que la tasa de acreción de masa sea máxima (Martí \& Paredes, 1995). El observatorio de rayos X Chandra ha permitido vislumbrar una posible emisión extendida de forma asimétrica, a una distancia de $\sim 0.1 \mathrm{pc}$ de LS I +61 303 (Paredes et al. 2007).

Los elementos orbitales de LS I +61303 fueron determinados por Casares et al. (2005b) quienes obtienen $e=0.72 \pm 0.15$. Para una inclinación $i=30^{\circ}$, las masas que se infieren son de $\sim 12 \mathrm{M}_{\odot}$ para la estrella, $\mathrm{y} \sim 2.5 \mathrm{M}_{\odot}$ para el objeto compacto. Si la inclinación es mayor, se obtiene una masa menor para el objeto compacto, de modo que puede tratarse de un agujero negro o una estrella de neutrones. Casares et al. (2005b) también han señalado que el espectro óptico de LS I +61303 es consistente con un aporte del $\sim 65 \%$ a la emisión visual por parte de la estrella (B0 Ve), mientras que la emisión restante se atribuye al material circunestelar.

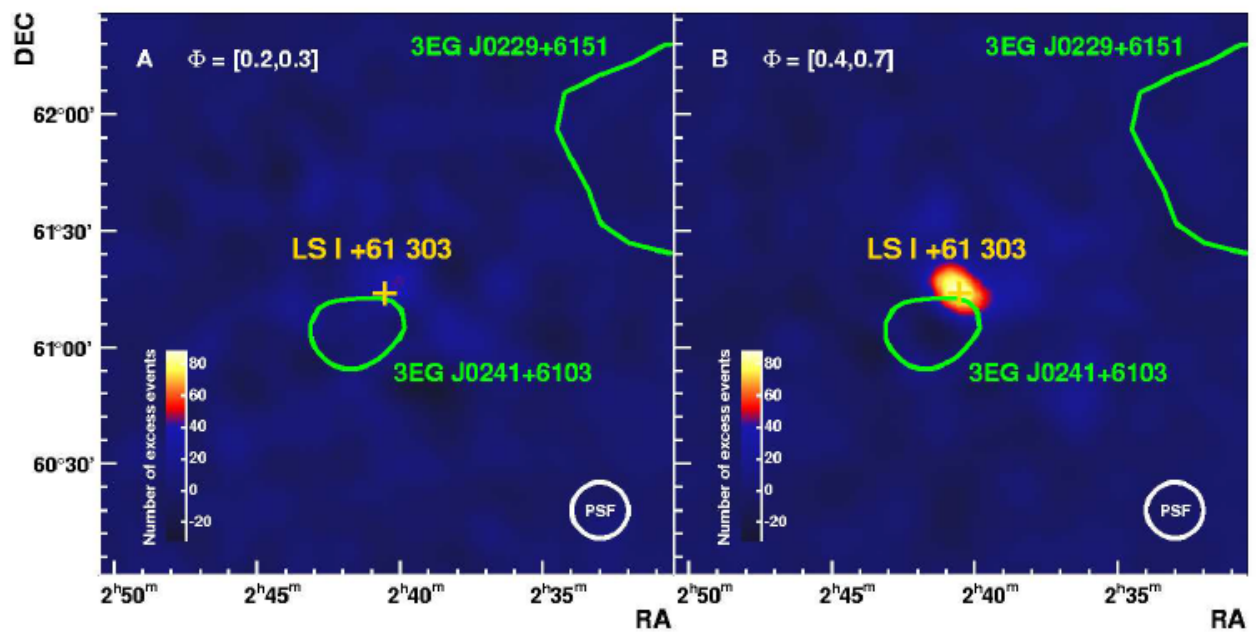

Figura 7.2: LS I +61 303 por MAGIC, Albert et al. (2006).

En 2006, el observatorio Cherenkov MAGIC detectót a LS I +61 303 como una fuente variable a energías muy altas (> $200 \mathrm{GeV}$, ver Albert et al. 2006). El máximo de la curva de luz en rayos $\gamma$ se produjo en fase $\phi=0.5-0.6$, y la fuente no fue detectada

\footnotetext{
${ }^{4} \mathrm{El}$ espectro en rayos $\mathrm{X}$ tiende a ser más blando cuando ocurren los aumentos de intensidad (Chernyakova et al. 2006).

${ }^{5}$ Valores alternativos por Grundstrom et al. (2007a) indican $e=0.55 \pm 0.05$.

${ }^{6}$ El telescopio Whipple sólo ha podido brindar cotas superiores al flujo en muy altas energías (Hall et al. 2003, Smith et al. 2007). Muy recientemente, VERITAS detectó flujo variable de LS I +61 303 a energías $>350 \mathrm{GeV}$, con máximo en fase $\phi \sim 0.65-0.74$, un poco después del pico detectado por MAGIC (Maier et al. 2007).
} 
durante el periastro (ver Figura 7.2). Se ajustó al espectro un índice $\alpha \sim 2.6$, y se ha estimado una luminosidad isotrópica $L_{\gamma} \sim 7 \times 10^{33} \mathrm{erg} \mathrm{s}^{-1}$. En observaciones contemporáneas, el pico de intensidad en radio $(15 \mathrm{GHz})$ ocurrió en fase $\phi=0.7$. La Figura 7.3 muestra la distribución espectral que resulta de recopilar la mayor parte de los datos disponibles (Sidoli et al. 2006). La luminosidad máxima se relaciona con la detección de COMPTEL, alcanzando $\sim 10^{35} \mathrm{erg} \mathrm{s}^{-1}$, para $E_{\gamma}$ en el rango $\mathrm{MeV}$.

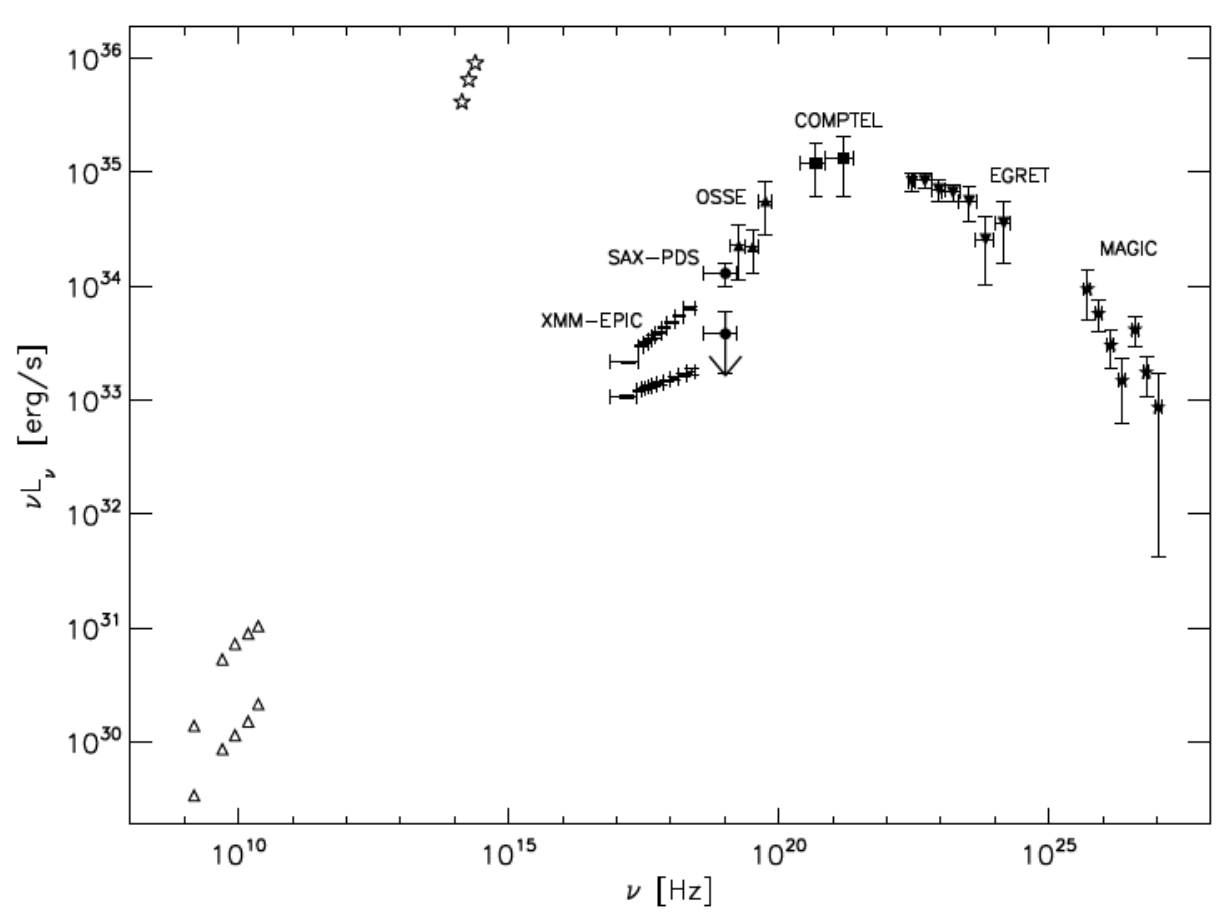

Figura 7.3: Distribución espectral de energía observada de LS I +61303 compilada por Sidoli et al. (2006). La emisión de la estrella Be es el máximo angosto en el rango visual-UV ( $\sim 3 \mathrm{eV})$.

El conjunto de datos disponibles hacen de LS I +61303 una fuente peculiar y han motivado diversas propuestas para explicar el origen de la emisión a lo largo de todo el espectro (ver por ej. Maraschi \& Treves, 1981; Taylor et al, 1992; Zamanov, 1995), pero aún hoy no se ha llegado a establecer en forma precisa cuál sería el escenario adecuado.

Varias propiedades de LS I +61 303 parecen ajustarse a un modelo de MQ. Si tal es el caso, se tiene una limitación energética dada por la cantidad de masa acretada por el objeto compacto por unidad de tiempo. Martí \& Paredes (1995), y luego Gregory \& Neish (2002) estimaron la tasa de acreción de masa (suponiendo un regimen de Bondi \& Hoyle) sobre el objeto compacto (expresión 4.1) obteniendo dos máximos para $\dot{M}_{\mathrm{ac}}$ : uno coincide con el periastro y el otro ocurre cuando es mínima la velocidad relativa entre el material del disco estelar y el objeto compacto (debido al alineamiento de los vectores velocidad). Estos máximos corresponden a eventos de acreción super-Eddington, durante los cuales se espera 
que se produzcan eyecciones en forma perpendicular al disco de acreción 7 . El modelo de MQ leptónico de Bosch-Ramon et al. (2006b) parece dar cuenta de las principales características de LS I +61 303 a lo largo de todo el espectrd 8 . Cerca del pasaje por el periastro se intensifican las pérdidas energéticas IC de los electrones, disminuyendo la probabilidad de una detección de emisión (sincrotrón) en radio. Asimismo, las pérdidas Coulombianas pueden ser importantes en las regiones internas de la órbita donde la densidad del viento estelar es mayor (Neronov \& Chernyakova 2007). A continuación se describe un modelo de MQ hadrónico para la emisión gamma de esta peculiar fuente.

\subsection{El modelo}

Basados en el escenario propuesto por Romero et al. (2003) que se describió en la Sección [5.2 se hicieron refinamientos para modelar la emisión gamma de LS I +61 303. En particular se tuvo en cuenta que en este MQ:

- La tasa de acreción de masa, y por tanto la potencia cinética de los jets, varía en forma significativa a lo largo de la órbita.

- El material del viento estelar que proporciona los protones blanco es la componente que forma el disco de decreción en torno a la estrella.

Para describir $\dot{M}_{\text {acc }}(\phi)$ se siguió el tratamiento dado por Martí \& Paredes (1995), suponiendo que el objeto compacto se encuentra inmerso dentro del disco de la estrella Be, y acreta material de éste en forma esférica 9 . Se consideró que el disco circunestelar tiene un perfil de densidad $\rho_{\mathrm{w}}(r)=\rho_{0}\left(r / R_{*}\right)^{-n}$, siendo $n=3.2$ y $\rho_{0}=5 \times 10^{11} \mathrm{~g} \mathrm{~cm}^{-3}$ (Gregory \& Neish, 2002). El gas del disco queda confinado cerca del plano ecuatorial, con un semi-ángulo de apertura $\sim 15^{\circ}$. Las ecuaciones de continuidad y conservación del impulso angular implican que la velocidad radial del material sigue $v_{r}=v_{r 0}\left(r / R_{\star}\right)^{n-2}$, mientras que la componente acimutal de la velocidad cae como $v_{\varphi}=v_{\varphi 0} R_{\star} / r$. Los valores de todos los parámetros adoptados se listan en la Tabla 7.1] y un esquema de la situación se muestra en la Figura 7.4

Las interacciones $p p$ pueden ocurrir ya sea porque se mezcla el material del viento con el del jet (por difusión), o porque algunos protones relativistas escapan del jet. El problema de intercambio de partículas a través de la superficie que limita a un jet relativista presenta

\footnotetext{
${ }^{7} \mathrm{Si}$ bien en la distribución espectral el hecho de que sea mayor la potencia en rayos $\gamma$ que en rayos $\mathrm{X}$ hace difícil pensar en un evento de acreción super-Eddington.

${ }^{8}$ Excepto por la ausencia de emisión térmica en rayos X.

${ }^{9}$ Cerca del objeto compacto el flujo acretado tiene una transición en su geometría y forma el disco de acreción. La conservación de la masa permite en principio utilizar la expresión de $\dot{M}_{\text {acc }}$ de Bondi-Hoyle. Además, aquí hemos supuesto que los cambios en la tasa de acreción de masa se propagan a la potencia cinética del jet de forma casi instantánea, o al menos en escalas de tiempo mucho más cortas que el período orbital.
} 
Tabla 7.1: Parámetros del modelo hadrónico para LS I +61 303. En la parte inferior se agruparon las características del disco circunestelar.

\begin{tabular}{|c|c|c|}
\hline Parámetro & Descripción [unidades] & valor \\
\hline$M_{\star}$ & masa de la estrella $\left[M_{\odot}\right]$ & 12 \\
\hline$R_{\star}$ & radio de la estrella $\left[R_{\odot}\right]$ & 10 \\
\hline$M_{\mathrm{BH}}$ & masa del objeto compacto $\left[M_{\odot}\right]$ & 2.5 \\
\hline$e$ & excentricidad & 0.72 \\
\hline$a$ & semi-eje orbital mayor $\left[R_{\star}\right]$ & 9.13 \\
\hline$i$ & inclinación del plano orbital $\left[^{\circ}\right]$ & 30 \\
\hline$\omega$ & ángulo del periastro $\left[^{\circ}\right]$ & 20 \\
\hline$\dot{M}_{\mathrm{w}}$ & tasa de pérdida de masa estelar $\left[M_{\odot} \mathrm{yr}^{-1}\right]$ & $1.3 \times 10^{-6}$ \\
\hline$R_{0}$ & radio del jet en la base $\left[R_{\mathrm{Sch}}\right]$ & 5 \\
\hline$z_{0}$ & altura de inyección del jet $\left[R_{\mathrm{Sch}}\right]$ & 50 \\
\hline$\chi$ & tangente del semi-ángulo de apertura del jet & 0.1 \\
\hline$\alpha$ & índice de potencia en la dist. de protones relativistas & 2.5 \\
\hline$\gamma /{ }_{p}^{\min }$ & factor de Lorentz mínimo de los protones & 2 \\
\hline$\Gamma$ & factor de Lorentz macroscópico del jet & 1.25 \\
\hline$T_{\star}$ & temperatura de la estrella $[\mathrm{K}]$ & 26000 \\
\hline$T_{\text {disk }}$ & temperatura del disco circunestelar $[\mathrm{K}]$ & 17000 \\
\hline$R_{\text {disk }}$ & radio externo del disco circunestelar $\left[R_{\star}\right]$ & 12 \\
\hline$R_{\text {in }}$ & radio de la región interna del disco circunestelar $\left[R_{\star}\right]$ & 3 \\
\hline$L_{\text {disk }}$ & luminosidad del disco circunestelar $\left[\mathrm{erg} \mathrm{s}^{-1}\right]$ & $2 \times 10^{37}$ \\
\hline$\psi$ & ángulo de apertura del disco circunestelar $\left[{ }^{\circ}\right]$ & 15 \\
\hline$v_{\mathrm{r} 0}$ & velocidad radial del viento en su base $\left[\mathrm{cm} \mathrm{s}^{-1}\right]$ & $3 \times 10^{5}$ \\
\hline$v_{\varphi 0}$ & velocidad acimutal del viento en su base $\left[\mathrm{cm} \mathrm{s}^{-1}\right]$ & $1.13 \times 10^{7} / \sin i$ \\
\hline$\rho_{0}$ & densidad del viento en su base $\left[\mathrm{g} \mathrm{cm}^{-3}\right]$ & $5 \times 10^{-11}$ \\
\hline
\end{tabular}

una dificultad más allá de los alcances de este trabajo; véase por ej. Hardee \& Hughes (2003), Stawarz \& Ostrowski (2002). Aquí se cuantificó el nivel de interacción a través de un "factor de mezcla", $f_{\mathrm{m}} \propto v_{\text {rel }}^{-1} \sim 0.1$. Esta prescripción fenomenológica da cuenta de un rechazo más eficiente de las partículas cuanto mayor es la velocidad relativa viento-objeto compacto, ya que se piensa que se intensifican los efectos de interfase. Para $\phi=0.5$, el factor de mezcla es máximo y alcanza $f_{\mathrm{m}} \simeq 0.5$.

El disco circunestelar se representó por un cuerpo negro con una temperatura efectiva $T_{\text {disk }}=17000 \mathrm{~K}$ en las regiones internas (hasta $\sim 3 R_{\star}$ ) donde se produce el grueso de la emisión IR, y más allá con una emisividad $\propto \rho_{\mathrm{w}}^{2}$ (Waters 1986, Bosch-Ramon et al. 2006b), extendiéndose hasta unos $\sim 12$ radios estelares. La emisión en la región interna del disco 
fue normalizada para obtener $L_{\text {disk }} \sim 2 \times 10^{37} \mathrm{erg} \mathrm{s}^{-1}$, en consistencia con los resultados de Casares et al. (2005b).

Se calculó la distribución espectral de energía para diferentes fases orbitales. En base a los resultados obtenidos, un valor del parámetro de acoplamiento $q_{j}^{\text {rel }} \sim 0.1$ es necesario para explicar la luminosidad de $7 \times 10^{33} \mathrm{erg} \mathrm{s}^{-1}$ inferida por MAGIC, para la fase $\sim 0.5$. En dicha configuración no se espera que los efectos de absorción $\gamma \gamma$ sean significativos, por lo que se adoptó el índice de la distribución de los protones relativistas como el mismo de la emisión detectada, $\alpha=2.5$. Este valor puede deberse a que el proceso de aceleración difusiva ocurre en frentes de choque con número de Mach bajo. De hecho, cabría esperar que la variación en la tasa de acreción de masa influya sobre el valor de $\alpha$ de modo que no se mantenga como un parámetro constante, o más aún, que los jets no sean estructuras completamente estables (Perucho \& Bosch-Ramon 2007).

Para los jets se supuso una geometría cónica. El campo magnético decae con la distancia como $\propto z^{-2}$, y en la base del jet es determinado por la condición de equipartición de la energía es $B_{0} \leq 10^{8} \mathrm{G}$ (ver Bosch-Ramon et al. 2006b). Como otras cantidades físicas, $B_{0}$

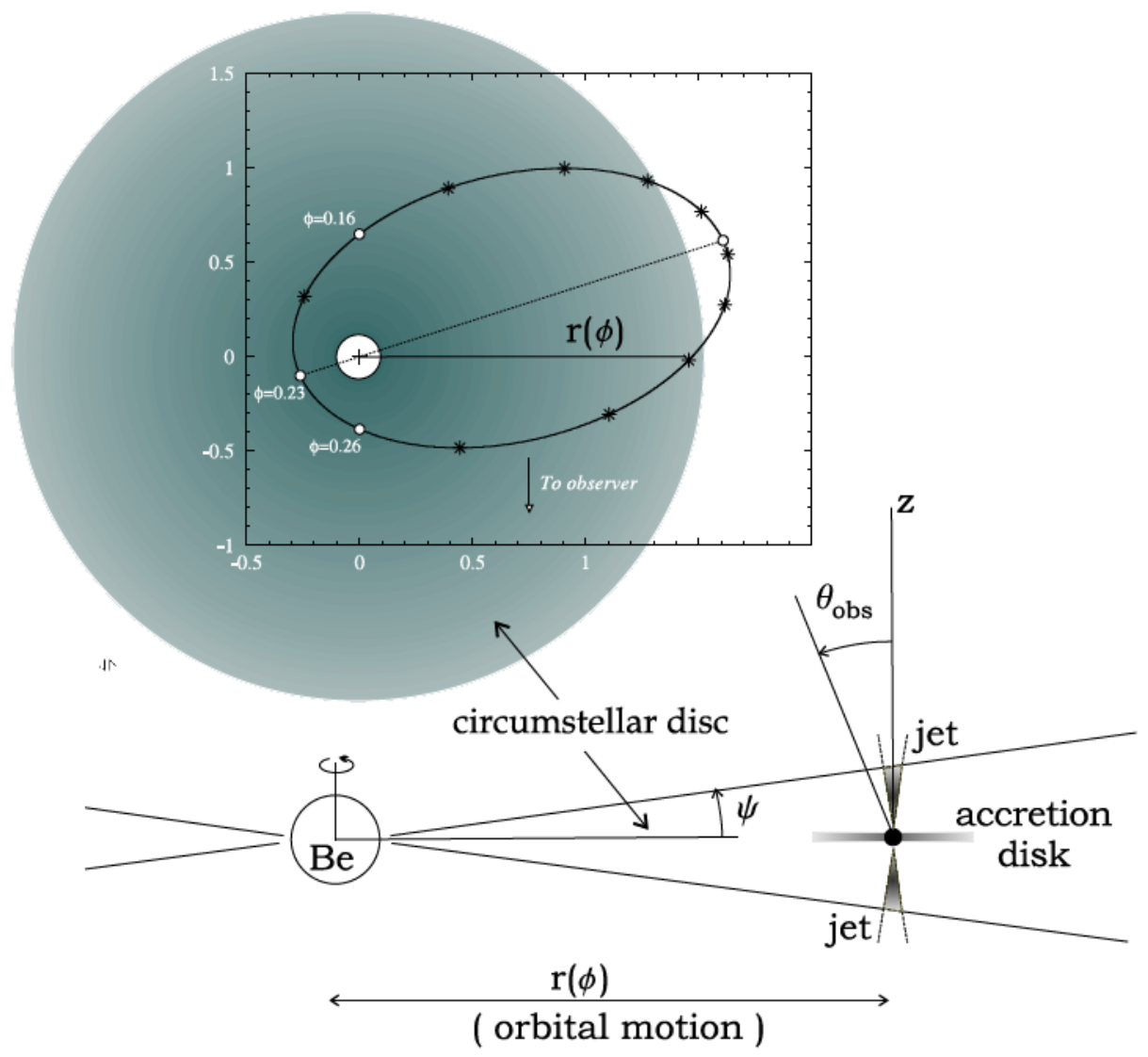

Figura 7.4: Esquema considerado para la geometría de LS I +61 303. La fase orbital se muestra en la vista superior y corresponde al mejor ajuste de Casares et al. (2005b). La unidad de distancia es el semieje mayor de la órbita. Abajo, fuera de escala, se esquematiza un corte transversal. 
cambia con la fase orbital, y es máximo en el pasaje por el periastro, alcanzando a ser tan intenso como para determinar la energía máxima de los protones $\sqrt{10}$ por pérdidas radiativas sincrotrón. Se obtuvo un factor de Lorentz máximo $\gamma_{p} \approx 7 \times 10^{6}$. No llegan a producirse interacciones foto-hadrónicas (creación de piones por $p+\gamma$ ) con el campo radiativo estelar, ya que no se supera la condición umbral. Aunque éste no es el caso si se considera a los rayos $\mathrm{X}$ que emite el disco de acreción, la luminosidad observada $L_{X}$ es lo bastante baja como para poder despreciar el efecto en una primera aproximación.

En la Figura 7.5 se muestra la curva de luz de la emisión hadrónica (línea punteada) calculada para fotones con energía $\sim 200 \mathrm{GeV}$, que es la mínima que reportan las observaciones de MAGIC. La emisividad diferencial se calculó aplicando la aproximación de la función $\delta$ (Aharonian \& Atoyan, 2000). La línea de trazo continuo en la misma figura corresponde a la luminosidad corregida por la absorción en el campo radiativo de la estrella y su disco de decreción. La profundidad óptica se calculó como en Dubus (2006c) para el término de la estrella. Para el disco estelar se consideró la expresión de la sección eficaz total, ya que el aporte principal (durante el periastro) se produce in-situ: los fotones gamma provienen mayormente de la base del jet, inmersa en el disco estelar donde se emiten los fotones térmicos que los absorben (ver esquema en la Figura 7.4, la separación durante el periastro entre estrella y objeto compacto es de $\left.\simeq 2.56 R_{\star}<R_{\text {disk }}\right)$.

Los fotones de la estrella absorben con mayor eficiencia a los fotones $\gamma$ de energía ligeramente mayor (algunos $\mathrm{TeV}$ ). Esto puede verse en el panel de la derecha de la Figura 7.5. donde se muestran los resultados de simulaciones Monte Carlo de cascadas electromagnéticas desarrolladas en el campo radiativo estelar, siendo $T_{\star}=26000 \mathrm{~K} \mathrm{y} R_{\star}=10$ $\mathrm{R}_{\odot}$. El código aplicado para estos cálculos es el que se describió en el capítulo anterior. El campo magnético en la región donde se producen las cascadas debe ser menor o similar a $0.1 \mathrm{G}$, para asegurar que las pérdidas radiativas de los electrones estén dominadas por las dispersiones IC. Las curvas fueron normalizadas con la luminosidad emitida en la fase correspondiente, dejando $q_{j}^{\text {rel }}$ como parámetro a ajustar a posteriori. Durante el periastro se espera un ablandamiento del espectro, siendo el índice $\alpha \sim 2.8$ en el rango de energías de la detección por MAGIC.

Puede notarse que un modelado más realista de las características del sistema generó algunos cambios en los resultados respecto de los reportados previament 11 en Romero et al. (2005). El valor que se obtiene, de $q_{j}^{\text {rel }} \sim 0.1$ para explicar la detección por MAGIC no parece exagerado cuando se tiene en cuenta la potencia inferida en otros jets potentes, como en Cyg X-1 (Gallo et al. 2005, ya comentado).

A energías por debajo de $\sim 1 \mathrm{GeV}$ el espectro de emisión seguramente recibirá aportes de origen leptónico (no calculados aquí), que cambiarán la forma del espectro, y podrían dar lugar a parte de la emisión detectada por EGRET. En Orellana \& Romero (2007) se

\footnotetext{
${ }^{10}$ Durante el resto de la órbita, la energía máxima de los protones está restringida por el radio de giro.

${ }^{11}$ Los cálculos presentados en Romero et al.(2005) son anteriores a la detección por MAGIC, aunque la predicen. Con posterioridad se refinó la elección de parámetros en base las restricciones impuestas por los nuevos datos observacionales.
} 

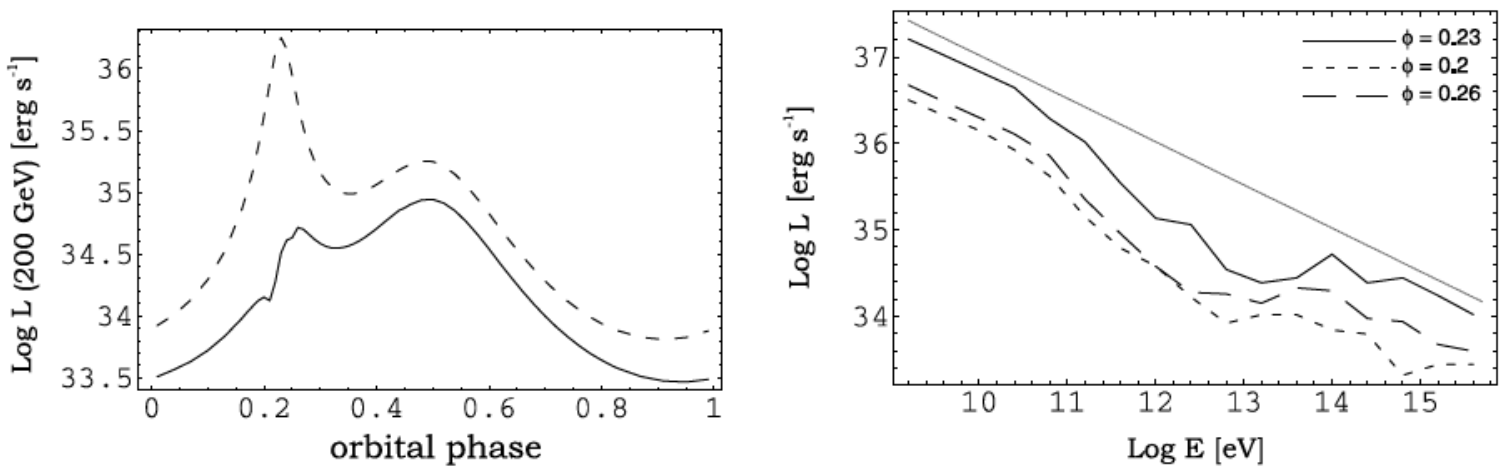

Figura 7.5: Izquierda: Curva de luz de la emisión hadrónica calculada para $E_{\gamma}=200 \mathrm{GeV}$. La línea punteada corresponde a la luminosidad emitida, mientras que la contínua tiene aplicada la corrección por absorción. Derecha: Distribución espectral resultante del reprocesamiento por cascadas electromagnéticas en el campo radiativo estelar. La recta indica la pendiente del espectro inyectado con índice espectral $\alpha=2.5$, y que se extiende hasta $\sim 1 \mathrm{PeV}$.

argumentó en favor del modelo de MQ hadrónico que, al igual que uno puramente leptónico, es capaz de explicar los datos observacionales. Para diferenciar el origen de la emisión a muy altas energías, sólo la detección de neutrinos que son un resultado secundario de las interacciones hadrónicas, puede ser conclusiva. En el caso de LS I +61303 , un modelo hadrónico de hecho prevee un flujo de algunos neutrinos por año por kilómetro cuadrado (Christiansen et al. 2006, y ver la discusión en Torres \& Halzen, 2007). Otra característica que permitiría obtener evidencia en favor de un modelo hadrónico para la emisión $\gamma$ se relaciona con la energía máxima (de cutoff) del espectro: en un modelo puramente leptónico las pérdidas energéticas llevan a una energía máxima de los fotones de alrededor de $1 \mathrm{TeV}$ (Bosch-Ramon et al. 2006b), mientras que en un modelo hadrónico como el descripto es posible que la emisión se extienda hasta energías mayores.

\subsection{Controversias}

En la sección anterior se describieron las características de un modelo de MQ para LS I +61 303. Algunos autores han propuesto que las propiedades de esta fuente pueden ser explicadas cuando se supone que el objeto compacto es una estrella de neutrones muy jóven, cuyo viento relativista interactúa con el viento de la estrella de gran masa, como ocurre en PSR B1259-63 (Sección 3.4). La primera versión de tal modelo fue propuesta por Maraschi \& Treves (1981), más tarde fue revisitado por Dubus (2006a). Los defensores de esta propuesta han invocado los resultados de observaciones en radio (Dhawan et al. 2006) como apoyo. Las observaciones en cuestión presentan imagenes VLBA, espaciadas por 3

\footnotetext{
${ }^{12}$ Existía el preconcepto de que, en vista de los aportes de MAGIC, se podía desechar un modelo de MQ hadrónico ya que las interacciones $p p$ que se verían intensificadas durante el periastro no parecían concordar con la no detección durante esa fase (Albert et al. 2006).
} 
días, cubriendo una órbita completa. Sólo cerca del pasaje por el periastro los contornos de densidad de flujo muestran una forma elongada que apunta aproximadamente en dirección opuesta a la de la estrella. No se observaron movimientos relativistas. Se ha interpretado a la estructura de forma variable detectada como como una especie de "cola cometaria" que resulta del choque de vientos. Sin embargo, tal modelo de colisión de vientos enfrenta serios problemas al intentar explicar la distribución espectral observada, el comportamiento de la curva de luz, y la propia morfología de la emisión en radio que pretende usarse como argumentd 13 .

Para evaluar en forma crítica las bondades de ambos escenarios hemos recurrido a métodos numéricos para la simulación dinámica tridimensional del frente de choque, en el caso de colisión de vientos, y por otro lado, de la interacción gravitacional que da lugar a la transferencia de masa. Estas simulaciones proporcionan estimaciones realistas de la tasa de acreción $\dot{M}_{\text {ac }}$ sobre el objeto compacto brinda nuevas perspectivas para un modelo de tipo MQ. Dichas simulaciones fueron llevadas a cabo por el Dr. Okazaki, y los resultados fueron discutidos en Romero et al. (2007).

\subsubsection{Simulaciones de colisión de vientos}

Para un valor razonable de la eficiencia para la emisión de rayos $\gamma$, la emisión inferida por EGRET de $\sim 10^{35} \mathrm{erg} \mathrm{s}^{-1}$ requiere que el viento del pulsar posea una potencia cinética $\dot{E}$ de al menos algunas veces $10^{36} \mathrm{erg} \mathrm{s}^{-1}$, en un escenario de colisión de vientos. Tal valor lleva a un flujo de impulso lineal que podrá ser compensado por el del viento estelar si éste tiene la energética apropiada. El parámetro más importante que determina la forma de la superficie de interfaz (o del frente de choque) es el cociente entre los flujos de impulso linea 14

$$
\eta=\frac{\dot{E}}{\dot{M}_{\mathrm{w}} v_{\infty} c},
$$

donde $v_{\infty}$ y $\dot{M}_{\mathrm{w}}$ son la velocidad y tasa de pérdida de masa de la estrella a través de la componente esférica del viento. $\mathrm{Si}$, por ejemplo, se toman valores para un viento fuerte en una estrella de tipo espectral B0V: $v_{\infty}=10^{3} \mathrm{~km} \mathrm{~s}^{-1}$ y $\dot{M}_{\mathrm{w}}=10^{-8} \mathrm{M}_{\odot} \mathrm{yr}^{-1}$, se tiene $\eta \sim 0.53 \dot{E} / 10^{36} \mathrm{erg} \mathrm{s}^{-1}$. En modelos bidimensionales simples (despreciando el movimiento orbital) la superficie puede aproximarse por un cono cuyo semi-ángulo de apertura es

$$
\theta=180^{\circ} \frac{\sqrt{\eta}}{1+\sqrt{\eta}}
$$

\footnotetext{
${ }^{13}$ Además, debe recordarse que no se han encontrado pulsaciones en la emisión en radio de LS I +61303 , que justifiquen la asociación del objeto compacto con un pulsar jóven. O bien un medio lo suficientemente denso diluye la pulsación, incluso en fases cercanas al apoastro, o debe suponerse alguna configuración geométrica particular para salvar este obstáculo. En cuanto al comportamiento de la curva de luz, se toma como posible responsable de la asimetría de la misma a la anisotropía con la cual es generado el viento del pulsar por las regiones polares del mismo, aunque aun no se han mostrado cálculos al respecto.

${ }^{14}$ Comparable con el planteo en la expresión (3.1).
} 
el cuál puede derivarse del análisis de Antokhin et al. (2004), donde se discute en detalle la forma de la superficie de interacción. Incluso para un valor mínimo de $\dot{E} \sim 10^{36} \mathrm{erg}$ $\mathrm{s}^{-1}$ se obtiene un cono muy ancho como para ser asociado con una cola cometaria, esto es $\theta \approx 62^{\circ}$. Rápidamente, al aumentar $\dot{E}$ el viento del pulsar sobrepasa al del viento estelar, dando $\theta>90^{\circ}$, lo que implica un cono cuyo vértice es la estrella y no el pulsar.

La situación se torna más compleja cuando se tiene en cuenta la deflexión asociada con el movimiento orbital, que es particularmente importante cerca del pasaje por el periastro, dada la excentricidad de este sistema. Para explorar los efectos del movimiento orbital sobre la superficie del frente de choque se realizaron simulaciones representando al viento estelar con los parámetros antes mencionados, y un flujo más rápido, que representa al viento del pulsar. Las simulaciones SPH realizadas no tienen la capacidad de tratar un flujo relativista 15 , por lo que se representó al viento del pulsar con una velocidad alta, $v_{\mathrm{PSR}} \sim 10^{4} \mathrm{~km} \mathrm{~s}^{-1}$ y se ajustó la tasa de pérdida de masa como para obtener el mismo flujo de impulso lineal que un flujo relativista, con la cantidad de energía total requerida. Esta aproximación nos permite estimar la forma de la superficie del frente de choque que es mayormente afectada por el balance de presiones entre los vientos colisionantes. Se ha despreciado en estas simulaciones la componente del viento estelar que forma el disco, ya que es muy improbable que ésta pueda producir una cola cometaria 16 . Por simplicidad, los efectos de calentamiento en las regiones post-choque, y de enfriamiento por procesos radiativos no fueron considerados. También por simplicidad, ambos vientos se supusieron isotermos y en expansión libre. Además, se desprecia la auto-gravedad de las partículas del viento, y se eligieron valores apropiados de los parámetros de viscosidad artificial. El plano orbital coincide con el plano $x$ - $y$ y el eje mayor de la órbita determina la orientación del eje $x$. Otros detalles técnicos se han incluído en Romero et al. (2007), pero no son relevantes aquí.

La Figura [7.6 muestra la distribución de la densidad en escala logarítmica, e ilustra la forma de la superficie de interfaz entre los vientos colisionantes de una estrella Be y un pulsar con una potencia de viento $\dot{E}_{\mathrm{PSR}}=10^{36} \mathrm{erg} \mathrm{s}^{-1}$, que corresponde a una razón de impulsos $\eta=0.53$. Aunque la superficie de interfaz muestra algunas variaciones debidas a inestabilidades, es fácil trazar la forma global, la cuál pone en claro el punto fundamental que se argumenta en contra del escenario de colisión de vientos: no hay "cola cometaria" durante el pasaje por el periastro y en ningún momento se producen morfologías "tipo jet".

\footnotetext{
${ }^{15}$ Los primeros resultados de estudios hidrodinámicos relativistas realizando un tratamiento bidimensional para el sistema de PSR B1259-63 (con colisión de vientos pulsar-estrella) se han publicado en forma posterior a nuestro trabajo (Bogovalov et al. 2007). En base a una comunicación personal, verificamos que nuestros resultados son consistentes con los reportados en dicho trabajo.

${ }^{16}$ De hecho, las simulaciones de interacción del objeto compacto con la estrella Be muestran que el disco circunestelar debe estar truncado, con un radio no superior a la separación en el periastro. Esto está en completo acuerdo observacional con las mediciones de $\mathrm{H}_{\alpha}$ de Grundstrom et al. (2007a).
} 

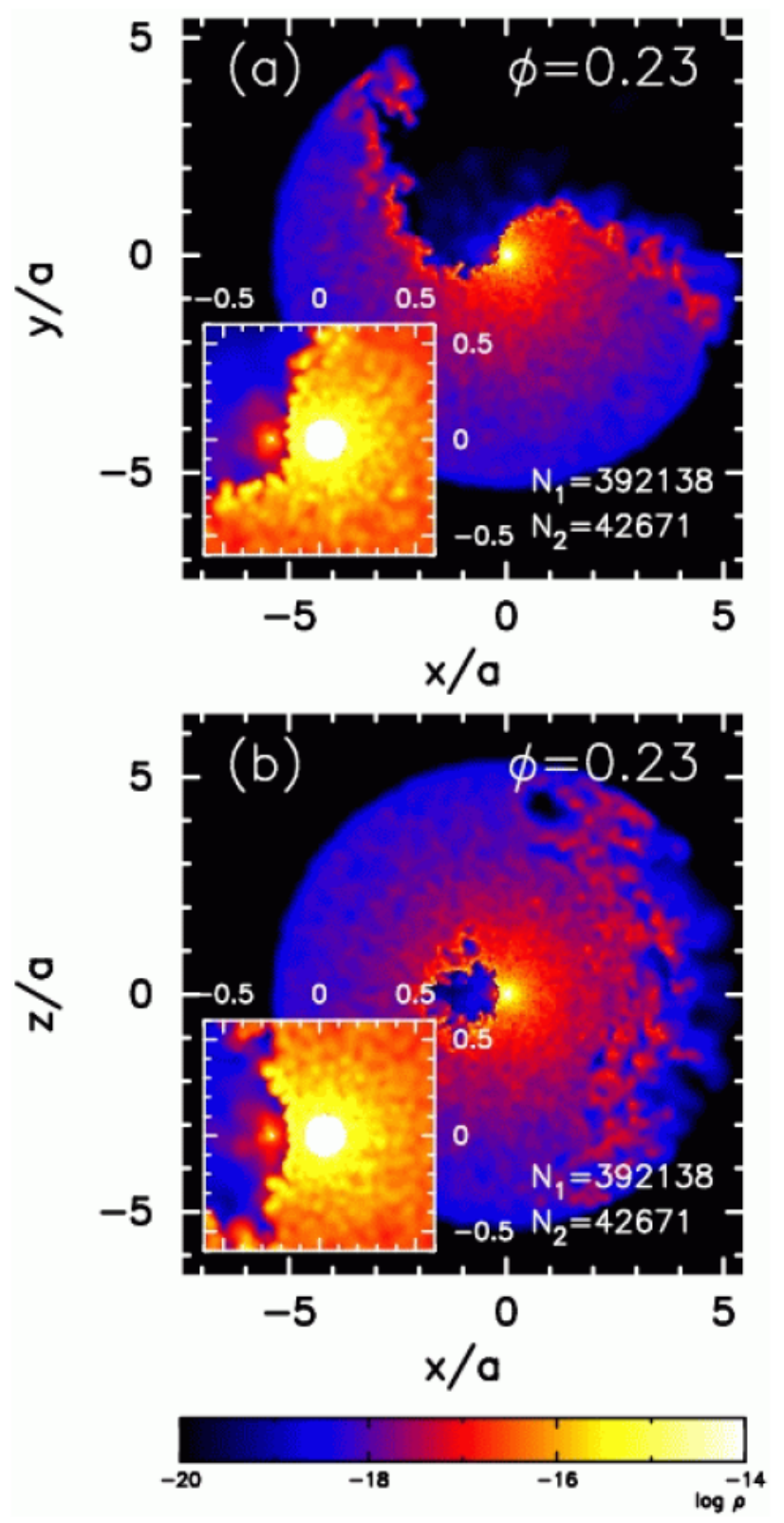

Figura 7.6: Forma de la superficie de interfaz entre los vientos colisionantes de una estrella Be y un pulsar, con los parámetros orbitales de LS I +61 303. La escala logarítmica muestra la densidad en (a) el plano orbital, y (b) el plano perpendicular y a lo largo del semije mayor de la órbita. Cerca del origen, en negro, se encuentra la estrella $\mathrm{Be}$, y el círculo oscuro más pequeño representa al pulsar. Se muestra ampliada la zona cercana a la estrella. La fase orbital, como se indica, es la del periastro. Las simulaciones SPH incluyen $N_{1}$ partículas de viento estelar, y $N_{2}$ del viento del pulsar. 


\subsubsection{Simulaciones de transferencia de masa}

Se ha simulado la evolución de la transferencia de masa en LS I +61 303 hasta que se alcanza un estado estacionario. El código aplicado es el descripto en Okazaki et al. (2002). Cada simulación demoró un promedio de 3 semanas utilizando supercomputadoras 17.

El disco de la estrella Be y el disco de acreción son modelados como un conjunto de partículas con auto-gravedad despreciable, mientras que la estrella y el objeto compacto son tratados como sumideros con su correspondiente masa gravitacional. El gas que cae dentro del radio de acreción es acretado por el sumidero. Se supone que el plano del disco de la estrella Be coincide con el plano de la órbita, que se elige como plano $x$ - $y$. Los parámetros adoptados para describir la estrella y el objeto compacto son los mismos que en la Tabla 7.1 consistentes con los obtenidos por Casares et al. (2005b). La escala de altura del disco circunestelar es $\sim 0.03 R_{\star}$ en $r=R_{\star}$, luego se incrementa hacia afuera como $r^{3 / 2}$. La densidad en la base de este disco es $5 \times 10^{-11} \mathrm{~g} \mathrm{~cm}^{-3}$.

Por motivos computacionales resultó conveniente separar la simulación de los dos discos. El disco circunestelar se considera isotérmo con $T_{\text {disk }}=0.6 T_{\star}$. Se supone una tasa constante de inyección de material justo fuera de la superficie estelar, que mayormente irá a formar el disco circunestelar. El material en las regiones externas de este disco se difunde hacia afuera, y resulta capturado por el objeto compacto si alcanza una distancia a éste menor que $\sim 0.9 r_{\mathrm{L}}$, donde $r_{\mathrm{L}}$ es el radio del lóbulo de Roche 18 . Las partículas que alcanzan esa condición son removidas de la simulación del disco circunestelar.

La segunda etapa de la simulación se enfoca en el disco de acreción alrededor del objeto compacto, utilizando como condición de contorno la tasa de transferencia de masa antes determinada (ver Hayasaki \& Okazaki, 2004). El borde interno del disco de acreción se elige como una fracción pequeña del semieje mayor de la órbita $a=6.4 \times 10^{12} \mathrm{~cm}$, más allá de la cual se espera que la acreción proceda rápidamente (en una escala temporal mucho menor que la de la región simulada).

En la Figura 7.7 se muestran vistas de los discos en fases cercanas al pasaje por el periastro. Cada panel muestra la densidad superficial en escala logarítmica. El objeto compacto queda representado por una esfera con radio de acreción $r_{\mathrm{ac}}=2.5 \times 10^{-3} a$. La estrella es el círculo oscuro más grande. En fases orbitales posteriores la onda de densidad que se ha excitado en el disco circunestelar alcanza al objeto compacto dando un máximo para la tasa de acreción de masa alrededor de fase $\phi \sim 0.5$.

\footnotetext{
${ }^{17}$ Se utilizaron computadoras HITACHI SR11000 y SGI Altix 3700, pertenecientes al Hokkaido University Information Initiative Center y al Yukawa Institute of Theoretical Physics, Kyoto University, respectivamente.

${ }^{18} \mathrm{El}$ radio del lóbulo de Roche para una binaria con órbita circular es aproximadamente (ej., Paczynski 1971)

$$
r_{\mathrm{L}} \simeq 0.462\left(\frac{q}{1+q}\right)^{1 / 3} D
$$

donde $q=M_{\mathrm{X}} / M_{\star}$ es el cociente de masas, siendo $M_{X}$ y $M_{\star}$ respectivamente las masas del objeto compacto y de la estrella, y $D$ la separación del sistema.
} 


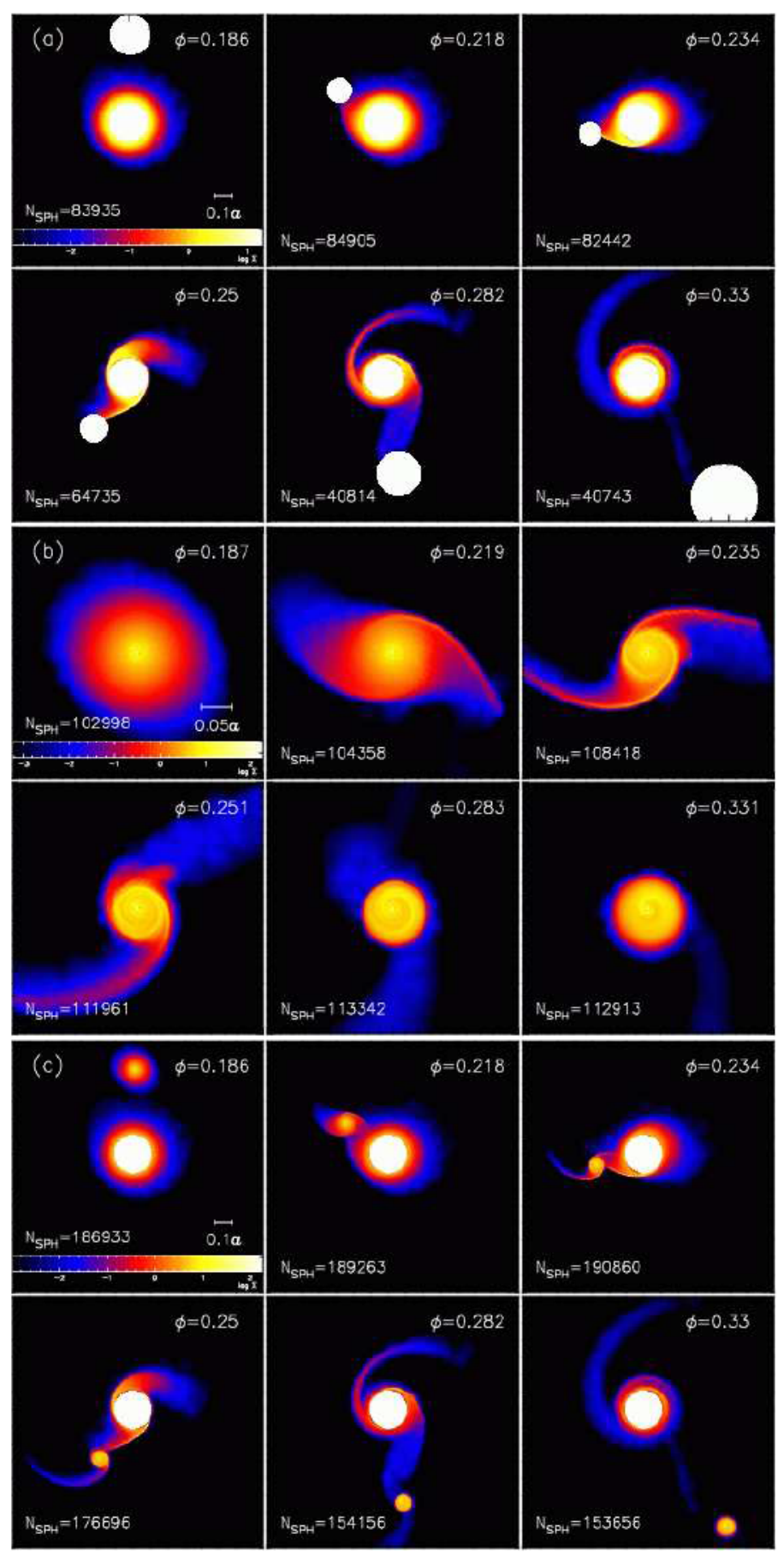

Figura 7.7: Resultados numéricos de simulaciones dinámicas: se muestra la evolución del disco de decreción de la estrella temprana en LS I +61 303 (panel $a$ ), y del disco de acreción en torno al agujero negro (panel $b$ ) en fases cercanas al periastro (Romero et al. 2007). Se ha graficado la densidad superficial en escala logarítmica. Se indica el número total de partículas SPH. El panel $c$ es un montaje de los anteriores. 
El principal objetivo de estas simulaciones fue el de comparar los resultados para la tasa de acreción con estimaciones analíticas, donde se asume que la acreción es esférica y ocurre al estar el objeto compacto inmerso dentro del disco circunestelar de la primaria. Como se ha comentado (Sección 4.1.2), esta aproximación es demasiado simplista en el sentido de no contemplar las perturbaciones al fluído del disco circunestelar que induce la interacción de marea entre las masas del sistema ni los efectos de la transferencia de masa. En la Figura 7.8 se ilustra la evolución con la fase orbital de la acreción de Bondi \& Hoyle y la obtenida mediante las simulaciones $\mathrm{SPH}$, una vez que se han promediado algunas órbitas para reducir el ruido (el comportamiento resulta regular luego de unas $\sim 30$ órbitas simuladas en total). A pesar de que la evolución de $\dot{M}_{\mathrm{ac}}(t)$ a lo largo de la órbita muestra aparentemente la misma disposición de máximos en ambos casos, los valores para la tasa de acreción de masa difieren notablemente ( $\sim 3$ ordenes de magnitud). Por otro lado, se corrieron simulaciones variando el radio de acreción $r_{\mathrm{ac}}$, de donde se deduce la posibilidad de que el pico de $\dot{M}_{\text {ac }}$ en el periastro sea un efecto computacional espurio, relacionado con la resolución de la simulación. El segundo máximo ha mantenido su forma y amplitud.
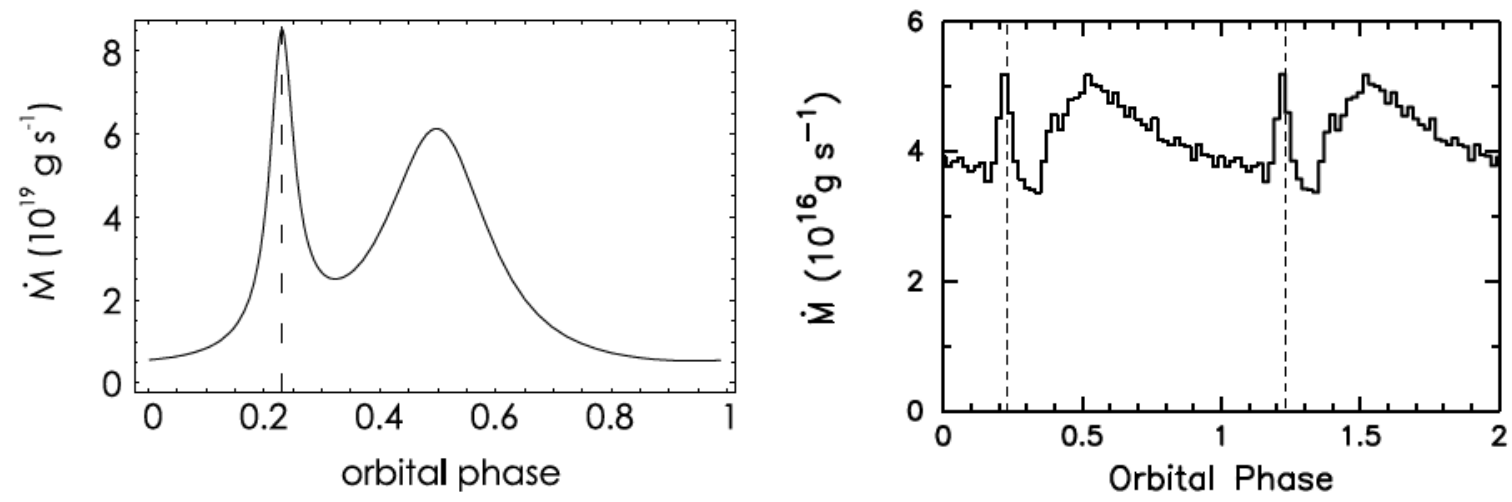

Figura 7.8: Tasa de acreción de masa. A la izquierda: en régimen de Bondi \& Hoyle, (reproduciendo Martí \& Paredes 1995). A la derecha: inferida de las simulaciones SPH (Romero et al. 2007). Se indica la fase de pasaje por el periastro.

Los resultados de simulaciones dinámicas indican que el pico en la evolución de $\dot{M}_{\text {ac }}$, que ocurre en fase $\phi \sim 0.5$ (retrasado del periastro y en forma coincidente con la detección de MAGIC) se debe a la propagación de una onda de densidad en el disco circunestelar que es inducida por la interacción de transferencia de masa. Se obtiene $\dot{M}_{\mathrm{ac}} \sim 5.2 \times 10^{16} \mathrm{~g} \mathrm{~s}^{-1} \rightarrow$ $\dot{M}_{\mathrm{ac}} c^{2} \sim 4.7 \times 10^{37} \mathrm{erg} \mathrm{s}^{-1}$, que podría proporcionar la potencia necesaria para que la emisión $\gamma$ a muy altas energías de LS I +61303 sea compatible con un escenario de microcuásar (ej. Bosch-Ramon et al. 2006b, y Orellana \& Romero 2007). Por ejemplo, en un modelo hadrónico donde $10 \%$ de la potencia acretada sea redirigida hacia los jets, y un $10 \%$ de ésta consista de protones relativistas, una estimación sencilla lleva a una luminosidad $\sim 8 \times 10^{34} \mathrm{erg} \mathrm{s}^{-1}$ debida a interacciones $p p$ con el material que rodea al objeto compacto.

El valor en fase 0.5 es $\dot{M}_{\text {ac }} \sim 0.01 \dot{m}$ donde $\dot{m}$ está expresado en unidades de la tasa de acreción de Eddington. Para $\dot{M}<0.05-0.1 \dot{m}$ existen soluciones para el flujo de acreción 

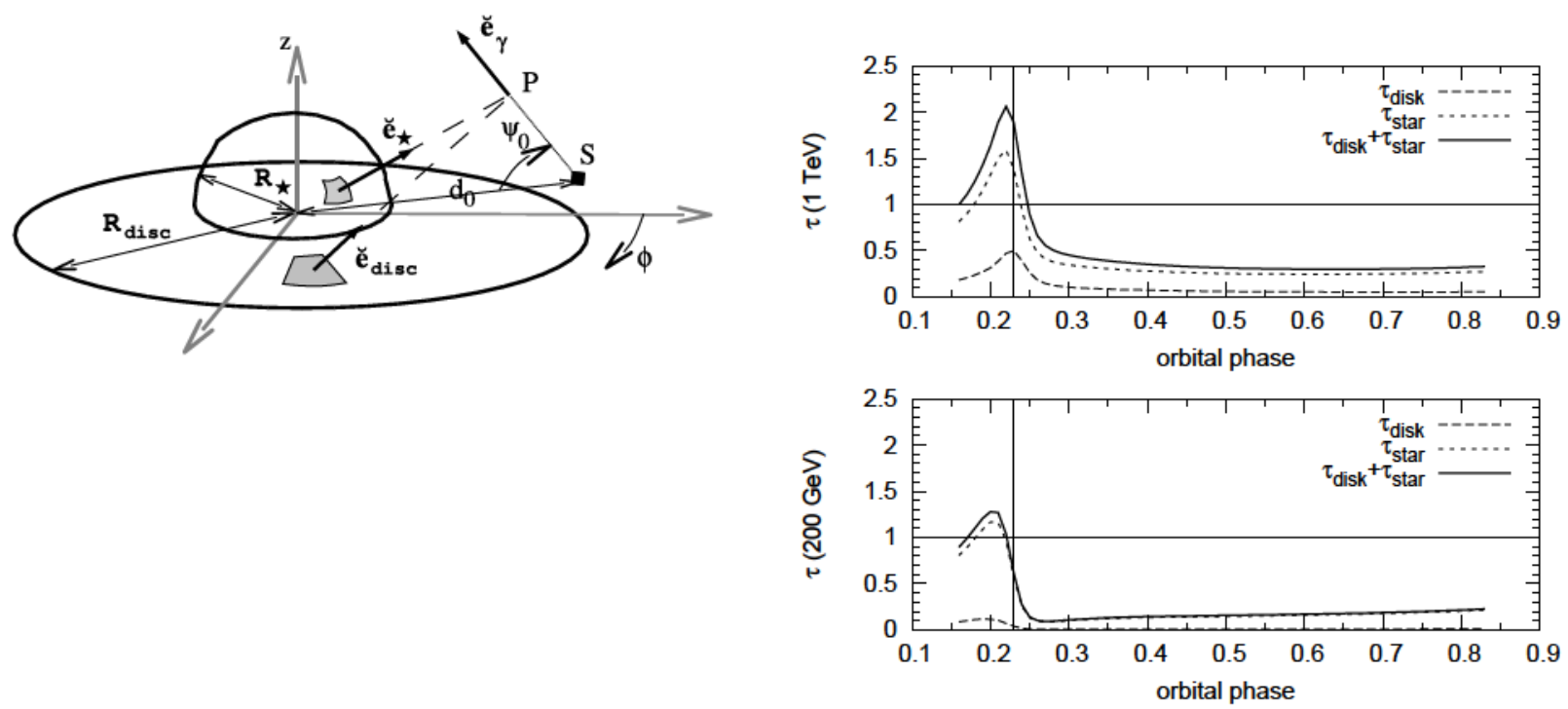

Figura 7.9: Izquierda: Geometría considerada para el cálculo de la opacidad a la propagación de rayos gamma por el campo radiativo de la estrella y su disco circunestelar. Derecha: resultados obtenidos para la evolución de la profundidad óptica con la fase, para dos valores fijos de energía.

dominado por advección (tipo ADAF, ver Narayan et al. 1998), que explicarían el débil brillo de LS I +61303 en rayos X.

Cerca del periastro, además de ser dudoso el valor de la tasa de acreción de masa real, los efectos de opacidad $\gamma \gamma$ en el campo radiativo estelar ciertamente disminuyen la posibilidad de detectar fotones $\mathrm{TeV}$ provenientes de LS I +61 303 durante dicha fase. En Romero et al. (2007) se presentan los valores de profundidad óptica recalculados para la geometría del disco circunestelar que se obtiene de las simulaciones SPH (ver Figura [7.9). Este disco tiene una caída abrupta en su perfil de densidad, por lo que se consideró $R_{\text {disk }} \sim 0.2 a \sim 1.8 R_{\star}$, siendo ésta la distancia a la cual la densidad superficial, $\Sigma$, cae en un factor $10^{-2}$ respecto del máximo. Se consideró al disco como un cuerpo negro 19 geometricamente delgado, con $T_{\text {disk }}$ y cuya emisión es $L_{\text {disk }} \sim 2 \times 10^{37} \mathrm{erg} \mathrm{s}^{-1}$. Los cálculos para estimar la opacidad del campo radiativo del disco circunestelar a la propagación de rayos $\gamma$ fueron adaptados de la descripción geométrica dada por Becker \& Kafatos (1995). Se obtuvieron valores menores que la unidad ( $\lesssim 0.5$ ), por lo que la principal fuente de absorción es el campo radiativo de la estrella. Cerca del periastro la profundidad óptica para fotones con energía $E_{\gamma} \sim 1 \mathrm{TeV}$ alcanza $\tau \sim 2$.

En definitiva, Romero et al. (2007) parece argumentar en favor de un modelo donde se produce la acreción/eyección de materia por parte del objeto compacto en LS I +61 303 . Sin embargo, el debate no queda cerrado y se espera que los datos observacionales que

\footnotetext{
${ }^{19} \mathrm{Si}$ bien los valores obtenidos para $\Sigma(r)$ parecen indicar que todo el disco es opticamente delgado a su propia emisión (y por tanto los fotones no están termalizados, ver Porter 1999), el cálculo detallado de la emisión del disco circunestelar nos alejaba del valor que infieren Casares et al. (2005b). Es por esto que se optó por una aproximación de cuerpo negro, ya que de todos modos las simulaciones realizadas se concentraban en la dinámica del gas y no eran tan confiables respecto de sus propiedades de emisión.
} 
proximamente provean AGILE y GLAST sean de fundamental ayuda para discernir la naturaleza de esta misteriosa fuente.

Simulaciones SPH: En general, las simulaciones SPH (sigla de Smoothed PARTICLE HYDRODYNAMICS) se utilizan para modelar flujos hidrodinámicos, incluyendo efectos gravitacionales. Este método divide al fluido en un conjunto de elementos discretos, partículas con separación espacial finita $h$ (llamada "smoothing length") sobre la cuál las propiedades son "suavizadas" por una función núcleo W (kernel). Esto hace que cualquier cantidad física de una partícula se pueda calcular sumando las propiedades relevantes de todas las demás partículas, pesando la contribución de cada una de acuerdo a su distancia con la partícula que interesa. Dicho peso es el que está gobernado matemáticamente por la función $W$, que suele ser de tipo Gaussiana o "spline".

Por ejemplo, para obtener la cantidad $A$ de la partícula $i$,

$$
A_{i}(r)=\sum_{j} m_{j} \frac{A_{j}}{\rho_{j}} W\left(r_{i}-r_{j}, h\right),
$$

donde $m_{j}$ es la masa de la partícula $j$ y $\rho_{j}$ la densidad asociada con ella. Suele fijarse un criterio para que $h$ sea adaptada por el programa de modo que la resolución espacial se incremente en zonas de mayor detalle. Estimadas las propiedades (contínuas) del fluido se puede seguir su evolución, que está dominada por el conjunto de ecuaciones hidrodinámicas y termodinámicas que corresponda. Para esto último, se fija también un paso temporal. 
CAPÍtulo 7. Un modelo hadrónico para la EMisión $\gamma$ DEL MiCrocuÁSAR LS I +61 303 
$\prod_{\text {CAPÍTULO }} \bigcirc$

\section{Emisión de leptones secundarios}

Hasta aquí se han comentado las características de un modelo de emisión hadrónica (primaria) para MQs de gran masa. Los procesos que dan lugar a dicha emisión generan también poblaciones leptónicas (secundarias) cuya radiación hemos investigado. Por un lado, y con mayor generalidad, se produce emisión leptónica al desarrollarse cascadas electromagnéticas en el campo radiativo estelar, tema del cual se trató en el Capítulo 6] Por otro lado, las interacciones inelásticas $p p$ dan lugar a la creación de neutrinos y leptones, a través de los decaimientos de los piones cargados y muones. En detalle, para protones con energía mayor que la umbral $E_{\text {th }}=1.22 \mathrm{GeV}$, a partir de 1

$$
p+p \rightarrow p+p+\xi_{\pi^{0}} \pi^{0}+\xi_{\pi^{ \pm}}\left(\pi^{+}+\pi^{-}\right)
$$

donde $\xi_{\pi}$ es la correspondiente multiplicidad (Mannheim and Schlickeiser 1994):

$$
\xi_{\pi} \simeq\left[\left(E_{p}-E_{\mathrm{th}}\right) / \mathrm{GeV}\right]^{1 / 4},
$$

tienen lugar los decaimientos:

$$
\pi^{+} \rightarrow \mu^{+} \nu_{\mu} \rightarrow e^{+} \nu_{e} \bar{\nu}_{\mu} \nu_{\mu} \quad \pi^{-} \rightarrow \mu^{-} \bar{\nu}_{\mu} \rightarrow e^{-} \bar{\nu}_{e} \nu_{\mu} \bar{\nu}_{\mu} \quad \pi^{0} \rightarrow \gamma \gamma
$$

En el ámbito de un MQ hadrónico, se genera así una población de pares secundarios que queda confinada dentro del jet debido a la presencia de campos magnéticos. Estos campos son probablemente muy turbulentos, por lo que es lógico suponer que en su movimiento los pares son rapidamente isotropizados después de haber sido creados.

Mientras que la emisión primaria se extiende hacia las altas energías a partir de $E_{\gamma} \gtrsim 1$ $\mathrm{GeV}$, a energías menores es la emisión secundaria 2 la que domina el espectro. Es en este rango del espectro donde puede buscarse evidencia alternativa de los procesos hadrónicos para, en última instancia, tratar de determinar el contenido material de los jets.

\footnotetext{
${ }^{1}$ Aunque menos probable, puede ocurrir también $p+p \rightarrow p+n+\pi^{+}+X$ y $p+p \rightarrow 2 n+2 \pi^{+}+X$, donde $X$ es una o varias partículas que en conjunto son neutras. Otra componente menor resulta de los canales que involucran mesones pesados, $\eta$ y kaones.

${ }^{2}$ Aquí no se han considerado leptones primarios, pero el caso real probablemente sea una mezcla.
} 


\subsection{Generación de leptones secundarios}

La cinemática de los decaimientos mencionados fue originalmente estudiada en detalle por Ginzburg \& Syrovatskii (1964). Para un flujo de protones relativistas que sigue una ley de potencias, $J_{p}\left(E_{p}\right)=K_{p} E_{p}^{-\alpha_{p}}$, los piones cargados y luego los leptones que resultan de la interacción con protones fríos, siguen también leyes de potencias. El índice espectral de la distribución de piones es

$$
\alpha_{\pi} \simeq \frac{4}{3}\left(\alpha_{p}-\frac{1}{2}\right)
$$

que para $\alpha_{p}$ cercano a 2 produce $\alpha_{\pi} \approx \alpha_{p}$. Los leptones resultantes conservan el mismo índice: $\alpha_{ \pm}=\alpha_{\pi}$, y el artículo citado brinda expresiones que relacionan las constantes de normalización.

En estimaciones más recientes se han considerado los procesos en un rango más amplio de energías 3 . Kelner et al. (2006) han obtenido expresiones para la tasa de inyección de pares (leptones por unidad de energía, volumen y tiempo) en función de la fracción de energía transferida $x=E_{e} / E_{p}$. Si la densidad numérica de protones blanco es $n(\bar{r})$, se tiene

$$
q_{e}\left(E_{e}, \bar{r}\right)=c n(\bar{r}) \int_{0}^{1} \sigma_{p p}\left(E_{e} / x\right) J_{p}\left(E_{e} / x\right) F_{e}\left(x, E_{e} / x\right) \frac{d x}{x},
$$

donde la sección eficaz inelástica también ha sido parametrizada por los autores:

$$
\sigma_{p p}\left(E_{p}\right)=\left(34.3+1.88 L+0.25 L^{2}\right) \times\left[1-\left(E_{\text {th }} / E_{p}\right)^{4}\right]^{2} \mathrm{mb} .
$$

En esta expresión $L=\ln \left(E_{p} / 1 \mathrm{TeV}\right)$. El espectro de leptones resultante del decaimiento $\pi \rightarrow \mu \nu_{\mu}$ es $F_{e}\left(x, E_{e} / x\right)$ y para $E_{p} \gtrsim 0.1 \mathrm{TeV}$ y $x \gtrsim 10^{-3}$ queda descripto por

$$
F_{e}\left(x, E_{p}\right)=C_{1}\left(E_{p}\right) \frac{\left(1+C_{3}\left(E_{p}\right)(\ln x)^{2}\right)^{3}}{x+0.3 x^{\left(1-C_{2}\left(E_{p}\right)\right)}}(-\ln (x))^{5},
$$

donde

$$
\begin{aligned}
C_{1}\left(E_{p}\right) & =\frac{1}{69.5+2.65 L+0.3 L^{2}}, \\
C_{2}\left(E_{p}\right) & =\frac{1}{\left(0.201+0.062 L+0.00042 L^{2}\right)^{1 / 4}}, \\
C_{3}\left(E_{p}\right) & =\frac{0.279+0.141 L+0.0172 L^{2}}{0.3+(2.3+L)^{2}} .
\end{aligned}
$$

En nuestra investigación, al aplicar esta aproximación, el espectro de los leptones resultantes pudo describirse como un espectro de ley de potencias, para un espectro original de protones que sigue también una ley de potencias.

\footnotetext{
${ }^{3}$ Los cálculos de Kelner et al. (2006) involucran integraciones numéricas muy complejas de las secciones eficaces diferenciales medidas en aceleradores de partículas, y resultados obtenidos mediante simulaciones numéricas de interacciones $p p$. Los autores buscaron aproximaciones analíticas que provean una precisión razonable.
} 


\subsubsection{Evolución de la población de leptones}

En general, la evolución de una población de partículas queda descripta por una ecuación de difusión (ej. Longair 1994) para la densidad numérica $N(E)$ de partículas con energía en el rango $E$ a $E+d E$,

$$
-\frac{d N(E)}{d t}=-D \nabla^{2} N(E)-\frac{d}{d E}[b(E) N(E)]-q(E) .
$$

En esta ecuación $D$ es el coeficiente escalar de difusión, $q(E)$ contiene el "término de fuente" que da cuenta de la producción de partículas con energía $E$ y posibles "sumideros", y $b(E)=-(d E / d t)$ es la tasa de pérdida de energía. Es claro que las funciones $b(E)$ y $q(E)$ pueden ser muy diferentes dependiendo de la naturaleza de las partículas consideradas. Para un estado estacionario, $\partial N(E) / \partial t=0$. Formalmente, la ecuación (8.8) se resuelve a través del uso de una función de Green.

En el contexto que nos interesa fue más conveniente la integración numérica dividiendo al jet en secciones cilíndricas de altura $\Delta z$, con tamaño reducido suficiente como para considerar que las cantidades físicas son uniformes. En tal caso, se anula el operador $\nabla^{2}$ y no tiene relevancia el coeficiente de difusión. La distribución de pares inyectados se verá afectada mayormente por pérdidas energéticas debidas a la emisión IC y sincrotrón, además de las pérdidas asociadas a la expansión adiabática. La tasa de pérdida de energía tiene los términos

$$
\begin{aligned}
&-\left(\frac{d E_{e}}{d t}\right)_{\text {sincr }}=\frac{\sigma_{T}}{4 \pi m_{e}^{2} c^{3}} B^{2} E_{e}^{2} \\
&-\left(\frac{d E}{d t}\right)_{\mathrm{IC}} \simeq c \sigma_{T} U_{\mathrm{ph}}\left(\frac{E}{m_{e} c^{2}}\right)^{2} \\
&-\left(\frac{d E}{d t}\right)_{\mathrm{exp}} \simeq \frac{2}{3} \frac{v_{\mathrm{exp}}}{R_{j}(z)} E,
\end{aligned}
$$

donde se introdujo la sección eficaz de Thomson $\sigma_{\mathrm{T}}=8 \pi e^{4} / 3 m_{e}^{2} c^{4} \approx 6.65 \times 10^{-25} \mathrm{~cm}^{2}$, y el campo magnético se mide en Gauss. La densidad de energía de los fotones estelares es $U_{\mathrm{ph}}=\frac{L_{\star}}{4 \pi c d^{2}}$, siendo $d$ la distancia desde la estrella y $L_{\star}$ su luminosidad. Y en la última expresión, $v_{\exp }=d R_{j}(z) / d t$ es la velocidad de expansión lateral del jet.

Por simplicidad, se desprecian efectos de re-aceleración. A una dada altura $z$ en el jet, continuamente ingresan nuevos electrones y positrones "frescos" a una tasa $q_{e}\left(E_{e}, z\right)$. Por otro lado, han avanzado hasta allí los que provienen de alturas inferiores y cuyo espectro ya ha sido modificado por las pérdidas de energía. Se mezclan así poblaciones que han "evolucionado" en distinto grado. Para realizar un cálculo consistente, se añade una ecuación de continuidad

$$
N_{e}\left(E_{e}, z\right) d E_{e}=N_{0, e}\left(E_{0, e}, z_{0}\right) d E_{0, e},
$$

donde la población inicial de partículas inyectadas es $N_{0, e}\left(E_{0, e}, z_{0}\right)=K_{0, e} E_{0, e}^{-\alpha_{e}}$. 


\subsection{Aplicación en un sistema similar a Cyg X-1}

Para aplicar este modelo en una configuración simple, hemos considerado un escenario similar al adoptado en Romero et al. (2003), donde el MQ tiene una órbita circular y el viento estelar tiene geometría esférica. Los parámetros adoptados para el sistema corresponden a los de Cyg X-1, que suele considerarse como el MQ arquetípicd 4 . Este sistema está formado por una estrella de $\sim 30 \mathrm{M}_{\odot}$ y un agujero negro de $\sim 10 \mathrm{M}_{\odot}$.

Para el cálculo de la emisión hadrónica primaria se procedió aplicando las expresiones de la Sección [5.2, fijando dos valores para la potencia de protones relativistas, $L_{p}^{\text {rel }}\left(Q_{j}\right.$ en la notación anterior), para considerar dos casos que rotulamos A y B. Específicamente, se consideró $L_{p}^{\text {rel }}=6 \times 10^{36} \mathrm{erg} \mathrm{s}^{-1}$ para el Modelo A, y $L_{p}^{\text {rel }}=6 \times 10^{35} \mathrm{erg} \mathrm{s}^{-1}$ para el Modelo B. Estos valores surgen de fijar la potencia $L_{p}^{\text {rel }}$ como una fracción constante $\eta$ de la luminosidad de Eddington del objeto compacto; siendo $\eta=0.005$ en el Modelo A, y $\eta=0.0005$ en el B. En la Figura 8.1 se muestra la luminosidad primaria generada a lo largo del jet (integrada en $z$ ), para diferentes direcciones $\theta$ respecto del eje del jet. Para obtener estos valores se ha utilizado la aproximación de la función $\delta$ para calcular la tasa de producción de piones neutros, y se utilizó la expresión (8.5) para la sección eficaz $p p$. En el cuadro pequeño se graficó el factor de beaming, $L(\theta) / L(\theta=0)$ que es independiente de la energía para $E_{\gamma} \gtrsim 10 \mathrm{GeV}$. La densidad de protones blanco del viento, en función de la distancia $r$ a la estrella se obtuvo a través de la ecuación de continuidad, siendo

$$
n(r)=\frac{\dot{M}_{\star}}{4 \pi m_{p} v_{\infty} r^{2}\left(1-R_{\star} / r\right)},
$$

donde se consideró $\dot{M}_{\star}=3 \times 10^{-6} \mathrm{M}_{\odot} \mathrm{yr}^{-1}$ y $v_{\infty}=2000 \mathrm{~km} \mathrm{~s}^{-1}$.

En la Figura 8.2 se muestra la modificación a la distribución espectral que tiene lugar debido al desarrollo de cascadas electromagnéticas en el campo radiativo estelar $\left(T_{\star}=3 \times\right.$ $\left.10^{4} \mathrm{~K}, R_{\star}=15 \mathrm{R}_{\odot}\right)$. Aquí se ha considerado que el sistema tiene una inclinación $i=30^{\circ}, \mathrm{y}$ se siguió la convención de fase utilizada por Szostek \& Zdziarski (2007) para Cyg X-1. Como punto representativo para el ingreso del espectro primario inyectado se utilizó una altura $z=a / 2$. Nos hemos remitido al caso en que el campo magnético estelar es bajo $(\lesssim 1 \mathrm{G})$ en la región donde se desarrollan las cascadas, asegurando que el mecanismo IC es el que domina las interacciones leptónicas en todo el rango de energías. Se implementaron simulaciones Monte Carlo similares a las antes descriptas. Una vez más, se ilustra el efecto esencial del desarrollo de las cascadas electromagnéticas para la redistribución de energía, aumentando en forma notoria la luminosidad a energías $E_{\gamma} \lesssim 100 \mathrm{MeV}$. Los resultados obtenidos

\footnotetext{
${ }^{4}$ La emisión gamma a muy altas energías de Cyg X-1 fue detectada recientemente por MAGIC (Albert el al. 2007). De hecho, la detección y variabilidad se anunciaron cuando nuestro trabajo ya se encontraba en referato. No fue nuestra intención predecir el comportamiento detallado de esta fuente, sino que simplemente fue elegida como caso de estudio.

${ }^{5}$ Notese que un campo magnético moderado, 1 Gauss, es suficiente para inhibir el desarrollo de las cascadas. Cálculos de emisión por enfriamiento sincrotrón de los pares inyectados por absorción $\gamma \gamma$ han sido realizados por Khangulyan et al. (2007).
} 


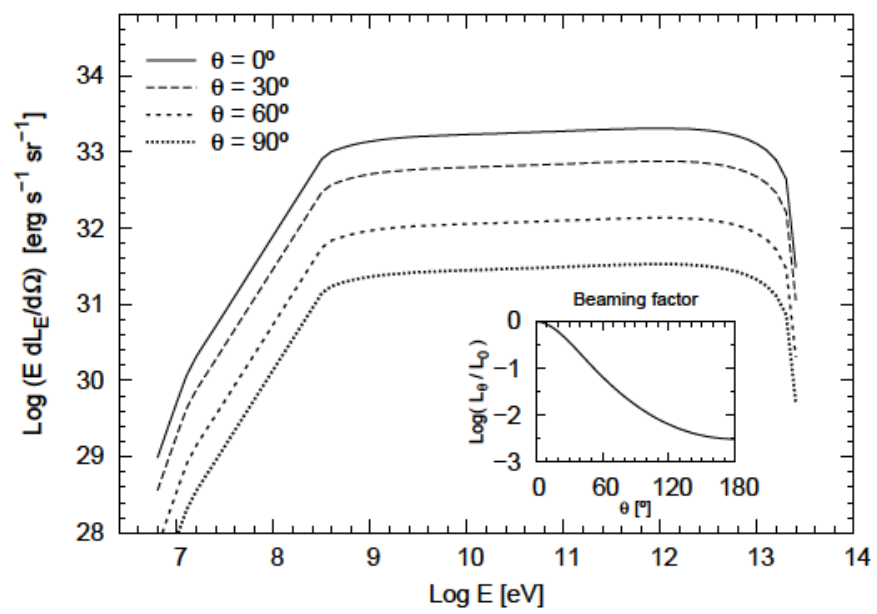

Figura 8.1: Luminosidad de rayos $\gamma$ producida por unidad de ángulo sólido por interacciones hadrónicas. Muestra, en orden decreciente, espectros correspondientes a $\theta=0,30,60$, y 90 grados, para el Modelo A $\left(L_{p}^{\mathrm{rel}}=6 \times 10^{36} \mathrm{erg} \mathrm{s}^{-1}\right)$.

apuntan hacia una anticorrelación entre los comportamientos de la emisión detectable en el rango de energías $\sim \mathrm{GeV}$ respecto del $\sim \mathrm{TeV}$.

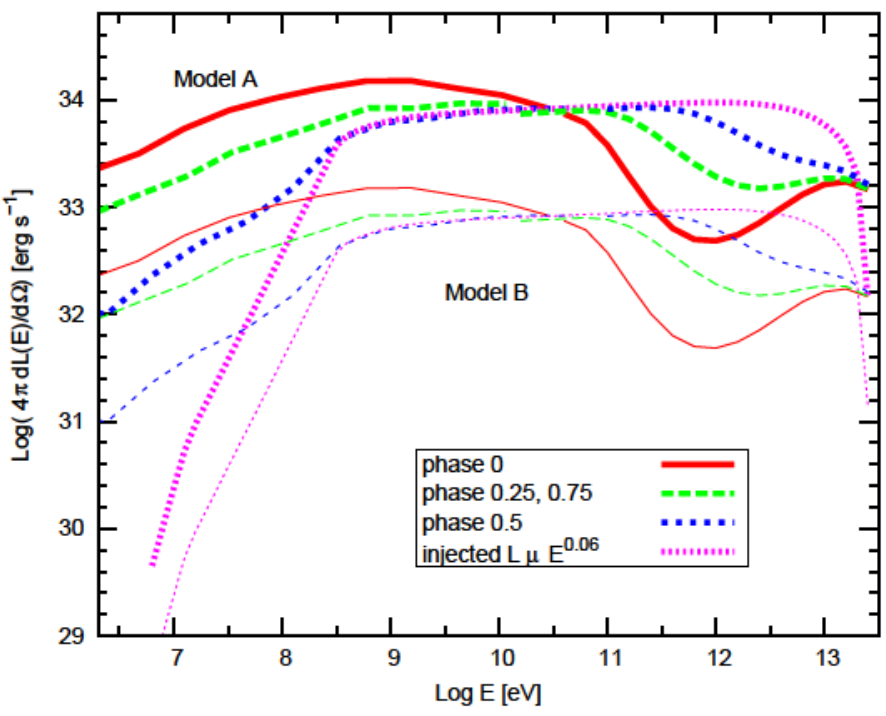

Figura 8.2: Luminosidad isotrópica resultante del procesamiento por cascadas electromagnéticas de la emisión $\gamma$ primaria en el campo radiativo estelar. Se adoptó una inclinación $i=30^{\circ}$, y la fase orbital tiene como referencia $\phi=0$ en la conjunción superior (esto es con objeto compacto detrás de la estrella).

Se realizó un cálculo consistente de la evolución de la población leptónica generada 
dentro de los jets por el decaimiento de piones cargados. Para esto, se procedió resolviendo la ecuación diferencial de pérdidas, como se comentó en la sección anterior. Esta etapa de computo numérico fue desarrollada por el grupo de altas energías de la Universidad de Barcelona, con el cual hemos colaborado. Nuestros cálculos les proveyeron de la tasa de inyección de pares (la dependencia en el ángulo $\theta$ se integró) y se obtuvo $q_{e}\left(E_{e}\right) \sim$ $3 \times 10^{28}\left(E_{e} / \mathrm{eV}\right)^{-1.92}$ leptones $\mathrm{erg}^{-1} \mathrm{~s}^{-1} \mathrm{~cm}^{-3}$ en la base del jet, para el Modelo A (un orden de magnitud menos para el Modelo B). Se consideró para el campo magnético $B_{0}$ un $10 \%$ del valor de equipartición en la base del jet, siendo $B_{0}=10^{5} \mathrm{G}$ (Modelo A) y $B_{0}=10^{4} \mathrm{G}$ (Modelo B). Por simplicidad para los dos modelos se fijó una sola energía máxima de los protones relativistas, $E_{p}^{\max } \sim 100 \mathrm{TeV}$, siendo $E_{e}^{\max } \simeq 0.04 E_{p}^{\max }$.

La emisión específica de los leptones resulta del proceso sincrotrón en presencia del campo magnético considerado, $B(z)=B_{0}\left(z_{0} / z\right)$, y radiación IC por interacción con fotones estelares. Los coeficientes específicos de emisión sincrotrón $\left(j_{\epsilon}(z)\right)$ y absorción $\left(k_{\epsilon}(z)\right)$ son los dados por Pacholczyk (1970). En el sistema de referencia del jet se calculó

$$
\frac{d L_{\epsilon}(z)}{d z}=2 \pi R_{\mathrm{j}}(z) \frac{j_{\epsilon}(z)}{k_{\epsilon}(z)} \times\left[1-\exp \left(-l_{\mathrm{j}} k_{\epsilon}(z)\right)\right]
$$

donde $l_{\mathrm{j}} \sim R_{\mathrm{j}}$ es el tamaño típico de la región emisora, y $\epsilon$ es la energía del fotón en unidades de $m_{\mathrm{e}} c^{2}$. El comportamiento de $d L_{\epsilon}(z) / d z$ con la altura, para la emisión radio en un caso similar, ha sido graficado en Bordas et al. (2007). Integrando a lo largo del jet se obtiene la distribución espectral de energía que en el sistema de referencia del observador es

$$
L_{\mathrm{syn}}^{\mathrm{obs}}=\epsilon \int_{z_{0}}^{z_{\max }} D^{2} \frac{d L_{\epsilon}}{d z} d z
$$

donde $D$ es el factor de Doppler que hemos definido en (4.7): $D=\left[\Gamma\left(1-\beta_{b} \cos \theta\right)\right]^{-1}$. Se calcula la emisión hasta un valor $z_{\max }$, que es algunas veces el tamaño del sistema binario.

Para la emisión IC, se ha considerado en una primera aproximación que las pérdidas de energía ocurren siempre en el regimen de Thomson, como lo indica la expresión dada en (8.9). Esto se fundamenta por dos hechos. En primer lugar, la expresión general para las pérdidas IC dada por Aharonian \& Atoyan (1981) vuelve inmanejable la ecuación de pérdidas para una resolución semi-analítica 6, como la que se implementó. Por otro lado, si bien a energías $E_{e} \gtrsim 10-100 \mathrm{GeV}$ las dispersiones tienen lugar en el regimen de Klein-Nishina con sección eficaz menor, este hecho sólo afecta el espectro IC emitido a las energías más altas aquí consideradas, donde la emisión primaria aporta el término dominante tapando por completo a la secundaria. Además, la mayoría de la emisión se genera en las proximidades de la base del jet, donde las pérdidas energéticas por mecanismo sincrotrón son más importantes que las IC y determinan la evolución de la población de pares.

La Figura 8.3 muestra las distribuciones espectrales sincrotrón e IC obtenidas. El conjunto de parámetros adoptados permitió ilustrar el impacto del balance entre las pérdidas

\footnotetext{
${ }^{6}$ Mientras que si es resoluble la ecuación incluyendo $-\left(\frac{d E_{e}}{d t}\right)=A E^{2}+B E$, donde $A$ y $B$ son constantes.
} 
energéticas IC y sincrotrón. La luminosidad sincrotrón alcanza valores $\lesssim 10^{32} \mathrm{erg} \mathrm{s}^{-1}$, y por debajo de la componente IC para ambos valores del campo magnético adoptado.

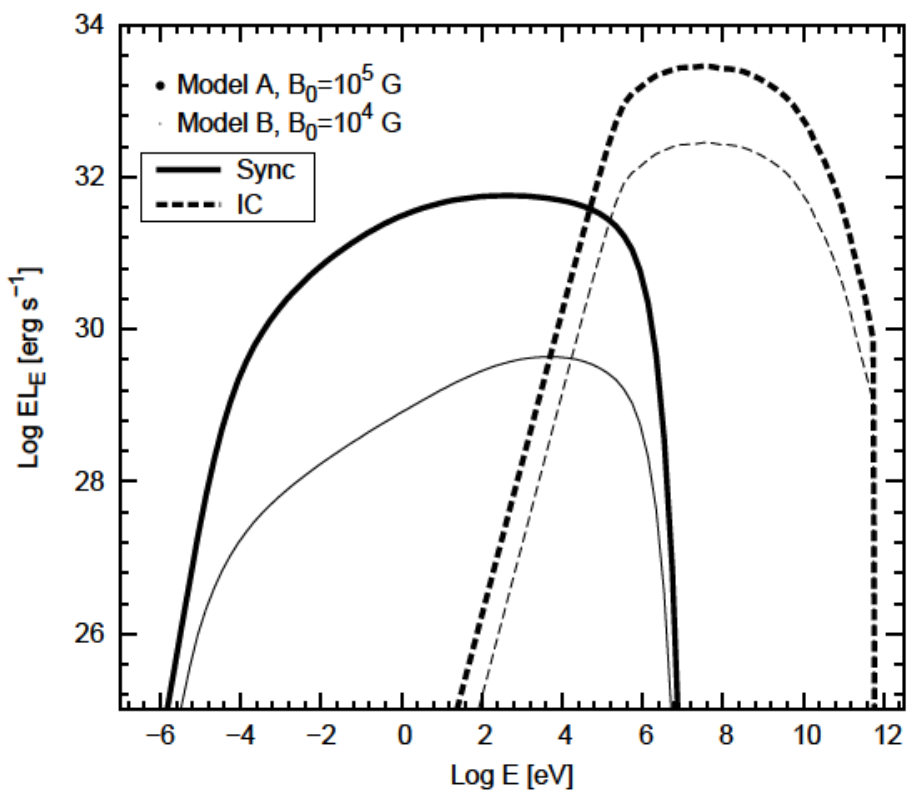

Figura 8.3: Distribución espectral de energía originada por pares secundarios dentro del jet (Orellana et al. 2007).

La cantidad total de energía radiada por secundarios, involucrados en las cascadas y dentro del jet es comparable a la emisión primaria resultado del decaimiento de piones neutros. Puede comentarse la detectabilidad de la emisión, cuando se asume una distancia típica $\sim 2$ kpc:

- En la banda de radio frecuencias, el espectro presentado es más bien duro y provee un flujo de a lo sumo una fracción de mJy, siendo sólo una parte pequeña de la señal detectada en MQs en el estado low-hard (ej, 15 mJy para Cyg X-1, Pooley et al. 1999). Si la contribución de los secundarios pudiera distinguirse, en alta resolución angular mostraría la forma del jet, rodeado por un halo de emisión que es el resultado (a más bajas energías que las calculadas) del reprocesamiento por cascadas. Al parecer, se hace necesaria entonces la presencia de una población de leptones primarios para poder explicar los flujos detectados en radio. Sin embargo, los leptones que no se han enfriado por completo y logran alejarse alcanzan regiones de condiciones más relajadas (campos magnéticos menores) como para continuar emitiendo en radio. Además, no hemos considerado en nuestros cálculos la posibilidad de que los leptones sean reacelerados a lo largo del jet, lo cual podría proveerles de la energía necesaria para explicar el espectro no térmico detectado. Tal posibilidad estuvo más allá de las suposiciones hechas en este trabajo, pero brinda una brecha interesante para ser explorada en el futuro. 
- En rayos X, la emisión generada por los leptones secundarios podría ser detectada por los instrumentos actuales, pero es probable que la emisión originada por el disco de acreción y/o la corona llegue a enmascarar hasta cierto punto esta contribución.

- La luminosidad obtenida en rayos X duros parece no ser suficiente para explicar los flujos detectados en algunos MQs. Sin embargo, aun no es claro si dicha radiación tiene un origen térmico o no térmico (ver ej. Markoff et al. 2005; Maccarone 2005; y también, Bosch-Ramon et al. 2006c).

- La radiación $\gamma$ en el rango $\mathrm{MeV}$ parece no ser despreciable, alcanzando luminosidades $\sim 10^{33} \mathrm{erg} \mathrm{s}^{-1}$. Este valor es aún bajo para ser detectado en una escala de tiempo razonable, con las capacidades de los instrumentos actuales. A energías más altas, el satélite GLAST o telescopios Cherenkov podrían detectar la emisión de la fuente, con su espectro suave debido al reprocesamiento por cascadas.

Incluso en el marco de un modelo simple como el que se ha expuesto, se ha encontado que en algunos rangos del espectro electromagnético la emisión presenta variabilidad. Muchos factores pueden llevar, en casos específicos, a variaciones más complejas (ej. Bosch-Ramon et al. 2006a). Por ejemplo, simplemente debido a la excentricidad de la órbita del sistema, puede inducirse una tasa de acreción de masa variable (como en LS I +61 303). Más impredecible, la naturaleza variable de los vientos estelares o su falta de homogeneidad también afecta la tasa de acreción. Otros posibles cambios pueden deberse a la precesión de los jets cuando no son perpendiculares al plano de la órbita (ej. Kaufman Bernadó et al. 2002). 


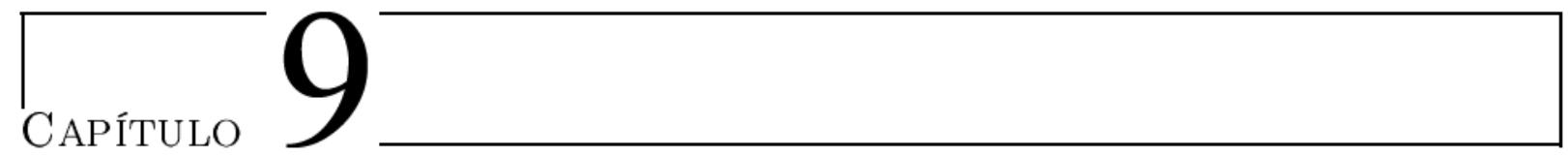

\section{Microcuásares con jets inclinados}

El eje de los jets y el plano orbital de un MQ no son necesariamente perpendiculares, aunque por simplicidad en estudios teóricos se suele suponer que si lo son, como en los casos expuestos hasta aquí. De hecho, Maccarone (2002) demostró que en sistema binarios, la escala de tiempo necesaria para que se produzca el alineamiento entre el spin de un agujero negro y el momento angular orbital es normalmente mayor que el tiempo de vida de la propia binaria1. Además, efectos de marea sobre el disco de acreción pueden inducir la precesión de los jets aumentando su inclinación respecto de la perpendicular del sistema (ej. Larwood 1998, Kaufman Bernadó et al. 2002). La precesión con una inclinación importante puede también ser un efecto de la deformación de las regiones internas del disco de acreción (ej. Wijers \& Pringle 1999, Ogilvie \& Dubus 2001) o deberse a interacciones spin-spin (ej. Bardeen \& Petterson 1975, Sarazin et al. 1980).

Como puede verse en la Tabla 4.1] existe evidencia observacional de la inclinación/precesión de los jets en varios MQs de gran masa. Entre ellos, el caso más notorio es el de V4641 Sgr para el cual se ha estimado que los jets se encuentran separados del plano orbital por sólo unos $\sim 35^{\circ}$ (ver Butt et al. 2003, y sus referencias). Otros sistemas donde los jets muestran precesión o variación en la inclinación incluyen a SS433 (el caso más firme), LS I +61 303, y Cyg X-3 (Katz 1980, Massi et al. 2004, and Mioduszewski et al. 2001, respectivamente). Se ha sugerido también que Cyg X-1 tiene jets precesantes (Romero et al. 2002).

Hemos considerado una aplicación del modelo hadrónico para el caso extremo en que el eje de los jets apunta en dirección muy próxima al plano orbital (Romero \& Orellana 2005), de modo que en forma periódica un jet se dirije directamente hacia la estrella. La Figura 9.1 esquematiza la situación, que no parece ser demasiado improbable. Por ejemplo, Butt et al. (2003) han considerado la nucleosíntesis inducida en la superficie estelar como consecuencia de la interacción con el jet que ocurre en tales sistemas. Nuestro trabajo mostró que a veces el jet no es capaz de llegar hasta la superficie de la estrella, siendo

\footnotetext{
${ }^{1}$ Intervienen efectos relativistas (de "arrastre de los sistemas inerciales") que también pueden tener lugar en las cercanías de una estrella de neutrones en rotación rápida.
} 


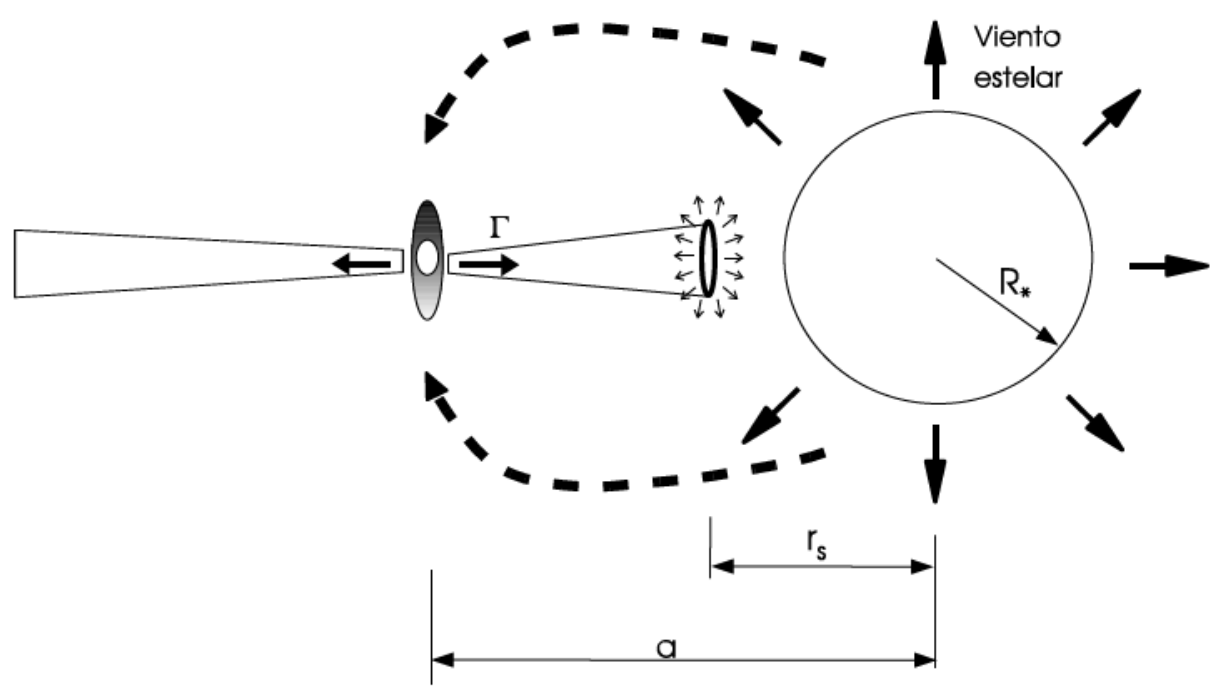

Figura 9.1: Esquema de la configuración tratada. En $r_{\mathrm{s}}$ el jet es frenado, ya sea por el viento o por la presión del campo magnético de la estrella de gran masa. Las líneas punteadas indican la transferencia de masa.

detenido antes por la presión del viento estelar. En el frente de choque resultante se reaceleran protones hasta muy altas energías, que luego pueden interactuar con el material del viento.

Aquí se contemplaron una variedad de condiciones para un MQ de alta masa con inclinación extrema. A tal fin se fijaron primero una serie de parámetros generales del sistema binario, considerando que lo forman un objeto compacto de masa $M_{\mathrm{BH}}=10 \mathrm{M}_{\odot}$, y una estrella de $M_{\star}=46.2 \mathrm{M}_{\odot}$ con un viento esférico importante, siendo $\dot{M}_{\mathrm{w}}=10^{-5} \mathrm{M}_{\odot}$ $\mathrm{yr}^{-1}$ la tasa de pérdida de masa, y $v_{\infty}=2500 \mathrm{~km} \mathrm{~s}^{-1}$ la velocidad terminal. La tasa de acreción de masa sobre el agujero negro es $\dot{M}_{\mathrm{ac}}=10^{-8} \mathrm{M}_{\odot} \mathrm{yr}^{-1}$, y el factor de Lorentz macroscópico de los jets, $\Gamma=10$. La separación orbital es $a=3 R_{\star}$, donde el radio de la estrella es $R_{\star}=15 \mathrm{R}_{\odot}$ (con estos valores queda asegurado que no se produce transferencia de masa por derrame del lóbulo de Roche).

A una dada distancia $r$ de la estrella la presión ejercida por el viento estelar es

$$
P_{\text {wind }}=\frac{\dot{M}_{\star} v(r)}{4 \pi r^{2}},
$$

donde $v(r)=v_{\infty}\left(1-R_{*} / r\right)$ es el perfil de velocidad del viento. Por otro lado, la presión asociada con el campo magnético de la estrella es

$$
P_{\mathrm{mag}}=B(r)^{2} / 8 \pi
$$

donde $B(r)=\sqrt{B_{\phi}^{2}+B_{r}^{2}}$. El campo magnético de la estrella es representado en forma estándar por un modelo de rotor magnético (Weber and Davis 1967; White 1985; Lamers 
and Cassinelli 1999):

$$
\begin{aligned}
\frac{B_{\phi}}{B_{r}} & =\frac{v_{\star}}{v_{\infty}}\left(1+\frac{r}{R_{\star}}\right) \\
B_{r} & =B_{\star}\left(\frac{R_{\star}}{r}\right)^{2} .
\end{aligned}
$$

Aquí $v_{\star} \simeq(0.1-0.2) v_{\infty}$ es la velocidad de rotación de la estrella en su superficie (Conti \& Ebbets 1977), y $B_{\star}$ el campo magnético estelar.

Por otro lado, si el jet se propaga con un ángulo de semi-apertura $\theta$ y a una velocidad de conjunto próxima a $c$, a una distancia $d$ del objeto compacto ejerce una presión dada por

$$
P_{\mathrm{j}}=\frac{L_{\mathrm{j}}}{\pi c \theta^{2} d^{2}} .
$$

En el caso de jets en AGNs, Bednarek \& Protheroe (1997) mostraron que un viento estelar intenso puede detener el flujo del jet antes que éste alcance la superficie de una estrella. La región de interacción queda formada por una discontinuidad de contacto que separa dos frentes de choque cuasi paralelos, propagándose en direcciones revertidas. La distancia $r_{\mathrm{s}}$ desde la estrella donde se sitúa la discontinuidad queda determinada igualando las expresiones (9.1) o (9.2) con la presión del jet, siendo $d=a-r$. Con los valores elegidos para el ejemplo (mencionados arriba), la presión del jet no puede ser equilibrada por la del viento si el parámetro de acoplamiento, que determina la potencia cinética del jet, es $q_{\mathrm{j}}>0.006$. Para valores mayores, la presión del campo magnético estelar puede sin embargo llegar a frenar el jet por balance de presiones. Por ejemplo, para $q_{\mathrm{j}}=10^{-2}$, un campo magnético $B_{\star} \geq 200 \mathrm{G}$ en la superficie de la estrella es necesario para que se forme un choque en la región entre el objeto compacto y la estrella 2 . Se eligieron distintos valores de $q_{\mathrm{j}}$ y de $B_{\star}$, para estudiar sistemas donde el jet llega a ser frenado.

Los frentes de choque generados por la interacción jet-viento estelar son sitios de reaceleración de partículas a través del mecanismo difusivo de Fermi (ver Apéndice). Para la reaceleración en un choque paralelo, la tasa de ganancia de energía de las partículas puede expresarse como (ej. Biermann \& Strittmatter 1987, Protheroe 1998):

$$
\left.\frac{d E}{d t}\right|_{\text {acc }}=\xi Z e c B, \text { donde } \xi \simeq 0.015(u / c)^{2},
$$

siendo $u$ la velocidad del frente de choque y todos los valores están en unidades cgs. Como en los casos vistos a lo largo de la tesis, la energía máxima que adquieren las partículas en el proceso queda impuesta por el balance entre ganancia y pérdidas de energía, o por el tamaño de la región de aceleración. Para los parámetros adoptados en este caso, el mecanismo que domina las pérdidas de energía de los protones son las interacciones $p p$ con

\footnotetext{
${ }^{2}$ Las estrellas de gran masa llegan a tener valores superficiales de campo magnético de hasta unos $\sim 10^{3}$ $\mathrm{G}$, de acuerdo a Donati et al. (2002).
} 
el material del viento. Para un protón relativista puede estimarse (Mannheim \& Schlickeiser 1994):

$$
-\left.\frac{d E}{d t}\right|_{p p} \approx 0.65 c n\left(r_{\mathrm{s}}\right) \sigma_{p p}\left(E-m_{p} c^{2}\right),
$$

donde $n\left(r_{\mathrm{s}}\right)$ es la densidad numérica de protones en el viento, cerca del frente de choque, y $\sigma_{p p}$ es la sección eficaz inelástica. La energía máxima de los protones resulta entonces

$$
E_{p}^{\max } \simeq \min \left[1.9 \times 10^{15}\left(\frac{u}{c}\right)^{2} \frac{B_{\mathrm{j}}\left(d_{o}\right)}{\mathrm{G}} \mathrm{eV}, 3 \times 10^{14} \frac{B_{\mathrm{j}}\left(d_{o}\right)}{\mathrm{G}} \mathrm{eV}\right]
$$

Se consideraron dos valores, $u / c=0.1$ para un caso sub-relativista, y $u / c=0.5$ para un choque relativista. En el primer caso $E_{p}^{\max }$ quedó determinada por las pérdidas y en el segundo por la cota del radio de giro, que se adoptó $\lesssim\left(a-R_{\star}\right)$. Los protones reacelerados siguen una distribución tipo ley de potencias, para la cual se consideró una energía mínima $E_{p}^{\min } \sim 10 \mathrm{GeV}$

$$
N_{p}(E)=K_{\alpha} E^{-\alpha}, 10 m_{p} c^{2}<E<E_{p}^{\max } .
$$

El índice $\alpha$ es $\sim 2$ para un choque no relativista fuerte (número de Mach alto), y $\alpha \sim 1.5$ para un choque relativista (ej. Protheroe 1998).

Una cierta fracción $\chi$ de la energía transportada por el jet se transfiere a las protones reacelerados en el frente de choque. Parece razonable adoptar una eficiencia $\chi=0.1$ (Blandford \& Ostriker 1978). Luego, la constante $K_{\alpha}$ en (9.8) puede despejarse de

$$
\chi L_{\mathrm{j}}=\left(a-R_{\star}\right)^{2} c \int_{10 m_{p} c^{2}}^{E_{p}^{\max }} N_{p}(E) E d E .
$$

\subsection{Difusión hacia las regiones internas del viento este- lar}

La población resultante de protones relativistas se difunde en el viento estelar, pero no todas las partículas son capaces de alcanzar las regiones más densas, cercanas a la estrella, donde es más probable que efectúen interacciones hadrónicas para generar rayos $\gamma$ (ver Romero \& Torres 2003, Torres et al. 2004). Que los protones puedan o no alcanzar la base del viento es una condición cuantificable a través del cociente $(\epsilon)$ entre las escalas de tiempo difusiva y convectiva. Respectivamente, estas escalas son

$$
t_{\mathrm{d}}=3 r^{2} / D, \quad \mathrm{y} \quad t_{\mathrm{c}}=3 r / v(r)
$$

donde $D$ es el coeficiente de difusión (Ginzburg \& Syrovatskii 1964):

$$
D \sim \frac{1}{3} \lambda_{r} c
$$


siendo $\lambda_{r}$ el camino libre medio para la difusión en la dirección radial. Aunque el valor del coeficiente de difusión no es una cantidad conocida, especialmente para un medio turbulento como es el del viento estelar, nos hemos guiado por las aproximaciones sugeridas por White (1985), y Völk \& Forman (1982). En la dirección paralela al campo magnético el camino libre es $\lambda_{\|} \sim 10 r_{\mathrm{g}}$, donde $r_{\mathrm{g}}=E / e B$ es el giro-radio para un proton de energía $E$ (manteniendo el sistema de unidades cgs). En la dirección perpendicular al campo el camino libre medio es menor: $\lambda_{\perp} \sim r_{g}$. En la dirección radial resulta entonces (Torres et al. 2004):

$$
\lambda_{r}=r_{g}\left(10 \cos ^{2} \theta+\sin ^{2} \theta\right),
$$

donde $\cos ^{-2} \theta=1+\left(B_{\phi} / B_{r}\right)^{2}$.

A través de la condición $\epsilon=1$ se encuentra la energía mínima de los protones, $E_{p}^{\min }(r)$, por debajo de la cual los protones son arrastrados lejos de la estrella por la convección 3 En el resto del trabajo, se adoptó $E_{p}^{\min }\left(r_{\mathrm{s}}\right)$ como la energía mínima de un protón que luego de haber sido acelerado en el frente de choque colisiona con un protón frío, cerca de la base del viento estelar.

\section{2. $\quad$ Producción y detectabilidad de rayos $\gamma \mathbf{y}$ neutrinos}

El flujo de protones relativistas es en este caso isotrópico, y se relaciona en forma simple con la densidad expresada en (9.8), de modo que $J_{p}(E)=c N_{p}(E) / 4 \pi$. La densidad de los protones del viento queda descripta por (8.11), y resulta $n\left(r \sim R_{\star}\right) \simeq 10^{15} \mathrm{~cm}^{-3}$, mientras que $n(r=a / 2) \simeq 10^{12} \mathrm{~cm}^{-3}$. El cálculo de la emisión de fotones $\gamma$ resultantes del decaimiento de piones neutros se realizó en el marco de la aproximación de momentos pesados por la distribución espectral (ver Gaisser 1990). Se consideraron diferentes modelos variando los parámetros $q_{j}, B_{\star}$ y $B_{j}\left(d_{0}\right)$, además de considerar los casos de choque relativista y sub-relativista ( $\alpha=1.5$ y $\alpha=2$, respectivamente). Las primeras tres columnas de la

\footnotetext{
${ }^{3}$ Explicitamente, Torres et al. (2004) proporcionan la siguiente expresión:
}

$$
\epsilon \sim\left\{3 e B_{\star} v_{\infty}\left(r-R_{\star}\right)\left(R_{\star} / r\right)^{2}\left(1+\left(v_{\star} / v_{\infty}\left(1+r / R_{\star}\right)\right)^{2}\right)^{3 / 2}\right\} /\left\{E_{p} c\left(10+\left(v_{\star} / v_{\infty}\left(1+r / R_{\star}\right)\right)^{2}\right)\right\} .
$$

${ }^{4}$ Este tratamiento de la sección eficaz diferencial lleva a

$$
q_{\gamma}\left(E_{\gamma}\right)=4 \pi \sigma_{p p}\left(E_{p}\right) \frac{2 Z_{p \rightarrow \pi^{0}}^{(\alpha)}}{\alpha} J_{p}\left(E_{\gamma}\right) \eta_{\mathrm{A}} \Theta\left(E_{p}-E_{p}^{\min }\right),
$$

donde el factor $Z_{p \rightarrow \pi^{0}}^{(\alpha)}$ es el llamado "momento pesado" y es $Z_{p \rightarrow \pi^{0}}^{(\alpha)}=\int_{0}^{1} x^{\alpha-1} F_{p \pi^{0}}(x) d x$, siendo $x=E_{\pi^{0}} / E_{p}$ y $F_{p \pi^{0}}(x)$ la sección inclusiva adimensional. El factor $\eta_{A} \sim 1.5$ tiene en cuenta la composición estándar del viento estelar. $\Theta$ es una función de Heaviside.

Este trabajo fue realizado con anterioridad a otros mencionados en esta tesis, utilizando una aproximación más simple para la emisividad $\gamma$. La aproximación de los momentos pesados, sin embargo, proporciona resultados compatibles con aproximaciones más recientes como las de Kelner et al. (2006), dentro de errores que no exceden el $10 \%$. 
Tabla 9.1 caracterizan cada modelo. Luego se dá la posición $r_{\mathrm{s}}$ del frente de choque, los rangos de energías de los protones relativistas que se difunden hasta las regiones internas del viento, y finalmente, la luminosidad $\gamma$ integrada. Los efectos de absorción en el campo radiativo estelar han sido considerados aplicando la expresión de la sección eficaz total $\sigma_{\gamma \gamma}$ (ver Figura 6.1), y se añadió un corte exponencial a altas energías. Las distribuciones espectrales resultantes se muestran en la Figura 9.2, para algunos modelos.

Tabla 9.1: Resultados de la emisión en altas energías generada en MQs con inclinación extrema. Las letras A, B, C en el nombre del modelo indican factor de acoplamiento alto, medio o bajo, respectivamente.

\begin{tabular}{cccccccccccc}
\hline Model & $q_{\mathrm{j}}$ & $\begin{array}{c}B_{\star} \\
{[\mathrm{G}]}\end{array}$ & $\begin{array}{c}B_{\mathrm{j}}\left(d_{0}\right) \\
{[\mathrm{G}]}\end{array}$ & $\begin{array}{c}r_{\mathrm{s}} \\
{\left[R_{\odot}\right]}\end{array}$ & $\begin{array}{c}E_{p}^{\min }[\mathrm{TeV}] \\
{\left[\begin{array}{c} \\
{[\mathrm{TeV}]}\end{array}\right.}\end{array}$ & $\begin{array}{c}E_{p, \mathrm{sub}}^{\max } \\
{[\mathrm{TeV}]}\end{array}$ & $\begin{array}{c}E_{p, \mathrm{mel}}^{\max } \\
{[\mathrm{TeV}}\end{array}$ & $\begin{array}{c}L_{\gamma,(\alpha=2)} \\
{\left[\mathrm{erg} \mathrm{s}^{-1}\right]}\end{array}$ & $\begin{array}{c}L_{\gamma,(\alpha=1.5)} \\
{\left[\mathrm{erg} \mathrm{s}^{-1}\right]}\end{array}$ & $\begin{array}{c}C_{\nu,(\alpha=2)} \\
{\left[\mathrm{erg}^{-1} \mathrm{~s}^{-1}\right]}\end{array}$ & $\begin{array}{c}C_{\nu,(\alpha=1.5)} \\
{\left[\mathrm{erg}^{-1} \mathrm{~s}^{-1}\right]}\end{array}$ \\
\hline$A 1$ & $10^{-2}$ & 500 & 10 & 21.7 & 92.2 & 190 & 3000 & $2.210^{32}$ & $4.110^{32}$ & $4.010^{32}$ & $1.710^{32}$ \\
$A 2$ & $10^{-2}$ & 500 & 100 & 21.7 & 92.2 & 1900 & 30000 & $7.510^{32}$ & $3.910^{32}$ & $3.310^{32}$ & $5.410^{31}$ \\
$B 1$ & $10^{-3}$ & 1 & 10 & 34.2 & 0.2 & 190 & 3000 & $1.910^{32}$ & $5.410^{31}$ & $3.710^{31}$ & $1.610^{31}$ \\
$B 2$ & $10^{-3}$ & 1 & 100 & 34.2 & 0.2 & 1900 & 30000 & $2.110^{32}$ & $4.310^{31}$ & $3.110^{31}$ & $5.410^{30}$ \\
$B 3$ & $10^{-3}$ & 100 & 10 & 34.2 & 21.3 & 190 & 3000 & $6.410^{31}$ & $4.710^{31}$ & $3.910^{31}$ & $1.710^{31}$ \\
$B 4$ & $10^{-3}$ & 100 & 100 & 34.2 & 21.3 & 1900 & 30000 & $1.110^{32}$ & $4.110^{31}$ & $3.310^{31}$ & $5.410^{30}$ \\
$C 1$ & $10^{-4}$ & 1 & 10 & 41.1 & 0.2 & 190 & 3000 & $1.910^{31}$ & $5.410^{30}$ & $3.710^{30}$ & $1.610^{30}$ \\
$C 2$ & $10^{-4}$ & 1 & 100 & 41.1 & 0.2 & 1900 & 30000 & $2.110^{31}$ & $4.310^{30}$ & $3.110^{30}$ & $5.410^{29}$ \\
$C 3$ & $10^{-4}$ & 100 & 10 & 41.1 & 20.8 & 190 & 3000 & $6.510^{30}$ & $4.710^{30}$ & $3.910^{30}$ & $1.710^{30}$ \\
$C 4$ & $10^{-4}$ & 100 & 100 & 41.1 & 20.8 & 1900 & 30000 & $1.110^{31}$ & $4.110^{30}$ & $3.310^{30}$ & $5.510^{29}$ \\
\hline
\end{tabular}
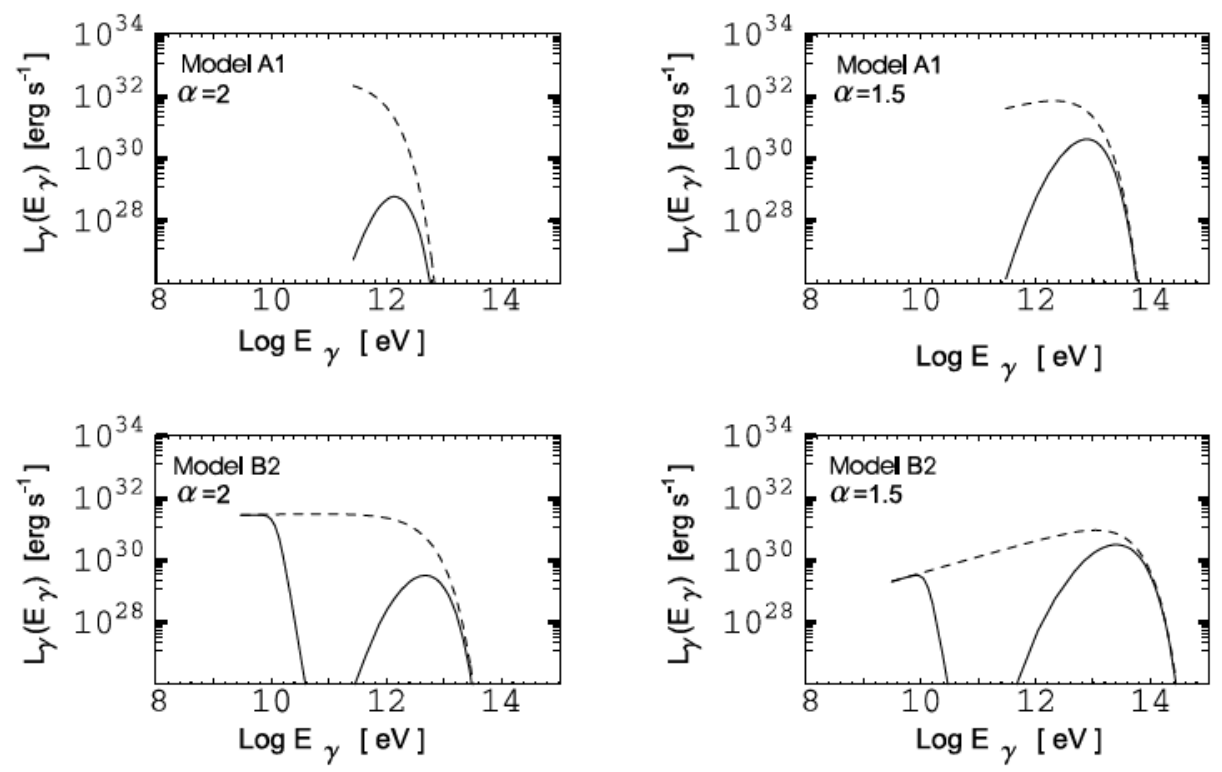

Figura 9.2: Distribuciones espectrales para la emisión $\gamma$ por interacción jet-viento en un modelo de MQ con inclinación extrema. La línea punteada corresponde a la producción, y la de trazo contínuo incorpora la opacidad del campo radiativo estelar (Romero \& Orellana 2005).

La intensidad $I_{\nu}\left(E_{\nu}\right)$ de neutrinos muónicos $\nu_{\mu}+\bar{\nu}_{\mu}$ puede derivarse de la distribución 
espectral de fotones $\gamma$ imponiendo una condición de conservación de la energía (AlvarezMuñiz \& Halzen 2002; Torres et al. 2004; y ver también Stecker 1979):

$$
\int_{E_{\gamma}^{\min }}^{E_{\gamma}^{\max }} E_{\gamma} I_{\gamma}\left(E_{\gamma}\right) d E_{\gamma}=C \int_{E_{\nu}^{\min }}^{E_{\nu}^{\max }} E_{\nu} I_{\nu}\left(E_{\nu}\right) d E_{\nu}
$$

donde $C$ es una constante numérica del orden de la unidad (si predominan las interacciones $p p$ por sobre $p \gamma$ ), de modo que la luminosidad total en neutrinos que produce el decaimiento de piones cargados es una cantidad comparable con la que se produce en fotones $\gamma$. La intensidad de neutrinos está dada por

$$
I_{\nu}\left(E_{\nu}\right)=C_{\nu}\left(E_{\nu} / \mathrm{erg}\right)^{-\alpha} e^{-E_{\nu} / E_{\nu}^{\max }},
$$

donde la constante $C_{\nu}$ tiene unidades de $\operatorname{erg}^{-1} \mathrm{~s}^{-1}$. Las energías de los extremos de la integral cumplen $E_{p}^{\max }=6 E_{\gamma}^{\max }$ y $E_{\nu}^{\max }=E_{\gamma}^{\max } / 2$. Los valores que se obtienen para los modelos propuestos se listan en las últimas columnas de la Tabla 9.1

Los neutrinos se producen en la región interna del viento estelar, como resultado de las interacciones $p p$. Una fracción de estos neutrinos, la cual depende de la geometría particular del sistema, será interceptada por la estrella. Si bien la estrella puede absorber una fracción de estos neutrinos, hemos estimado que la deposición de energía $\eta_{\nu}$ (ver Gaisser et al. 1986) en el interior estelar que estos procesos conllevan es muy pequeño como para generar un efecto de calentamiento observable.

Si la fuente se encuentra a una distancia $d_{\mathrm{MQ}}$, el flujo de neutrinos que escapan en dirección de la tierra es $F_{\nu}\left(E_{\nu}\right)=I_{\nu}\left(E_{\nu}\right) /\left(4 \pi d_{\mathrm{MQ}}^{2}\right)$. Para estimar si este flujo es detectable por un instrumento como ICECUBE hemos adoptado una distancia típica de una fuente galáctica, $d_{\mathrm{MQ}} \sim 5 \mathrm{kpc}$. La señal producida en el detector puede estimarse como (Anchordoqui et al. 2003)

$$
S=T_{\text {obs }} \int d E_{\nu} A_{\text {eff }} F_{\nu}\left(E_{\nu}\right) P_{\nu \rightarrow \mu}\left(E_{\nu}\right)
$$

donde $P_{\nu \rightarrow \mu}\left(E_{\nu}\right) \approx 1.3 \times 10^{-6}\left(E_{\nu} / \mathrm{TeV}\right)^{0.8}$ denota la probabilidad de producción de un muón cuando el $\nu$ atraviesa el detector, para $E_{\nu} \sim 1-10^{3} \mathrm{TeV}$ (Gaisser et al. 1995) Aquí, $T_{\text {obs }}$ es el tiempo de observación y $A_{\text {eff }}$ el área efectiva del detector. Por otro lado, el ruido está dado por

$$
N=\left[T_{\text {obs }} \int d E_{\nu} A_{\text {eff }} F_{\mathrm{B}}\left(E_{\nu}\right) P_{\nu \rightarrow \mu}\left(E_{\nu}\right) \Delta \Omega\right]^{1 / 2},
$$

donde $\Delta \Omega$ es el ángulo sólido del haz del detector $\left(\Delta \Omega_{1^{\circ} \times 1^{\circ}} \approx 3 \times 10^{-4}\right.$ sr para ICECUBE, Karle et al. 2002) y

$$
F_{\mathrm{B}}\left(E_{\nu}\right) \leq 0.2\left(E_{\nu} / \mathrm{GeV}\right)^{-3.21} \mathrm{GeV}^{-1} \mathrm{~cm}^{-2} \mathrm{~s}^{-1} \mathrm{sr}^{-1}
$$

\footnotetext{
${ }^{5}$ Estos autores calcularon una aproximación simple, donde $P_{\nu \rightarrow \mu}\left(E_{\nu}\right)$ sigue una ley de potencias quebrada en $E_{\nu}=1 \mathrm{TeV}$, la cual refleja la dependencia de la sección eficaz del proceso.
} 
es el flujo atmosférico de neutrinos $\nu_{\mu}+\bar{\nu}_{\mu}$ (Volkova 1980, Lipari 1993). Para el rango de operación del detector $\left(1-10^{5} \mathrm{TeV}\right)$ hemos calculado el tiempo de integración necesario para lograr $S / N=3$. Los resultados han sido demasiado grandes como para considerar la detectabilidad en una escala de tiempo razonable (miles de años para todos los casos, modelos A1 al C4), con la sensibilidad de los instrumentos actuales.

Los valores obtenidos son cotas inferiores, ya que la fuente es claramente variable. El período orbital (circular) del sistema que se discute es de $\sim 2.5$ días. La estrella abarca un $\sim 10 \%$ del cielo del objeto compacto, y al haber dos encuentros con los jets, se espera que el ciclo de actividad sea $\sim 20 \%$. La emisión $\gamma$ también se "prende" dos veces por órbita, cada $\sim 30$ horas, y la fuente permanece activa por unas $\sim 6$ horas cada ved 6 .

Dada la isotropización que ocurre en el frente de choque, estos modelos no serán tan dependientes del ángulo de inclinación como los de jet relativista puro (ej. Romero et al. 2003). Eventos de ocultación ocurrirían sólo si la visual coincide con el plano orbital, pero incluso así, el flujo no sería suprimido por completo ya que las partículas se difunden por toda la región interna del viento. Aunque aquí no hemos desarrollado el cálculo detallado, cabe esperar que el reprocesamiento por cascadas electromagnéticas iniciadas por los fotones $\mathrm{TeV}$ haga de los MQs inclinados fuentes variables en el rango $\mathrm{GeV}$ y $\mathrm{MeV}$.

\subsubsection{Caso de interacción directa con la superficie de la estrella}

Si la potencia del jet es suficiente, éste impacta directamente contra la estrella. Para ilustrar este caso, consideramos un último modelo con $q_{\mathrm{j}}=0.1$. El flujo de protones relativistas que interactúa con el material estelar es aquí el resultante de la aceleración a lo largo del jet, y sigue una distribución similar a la dada en (5.4), salvo que interesa el flujo que se aproxima a la estrella 8 Se consideró $E_{p}^{\prime \max }=100 \mathrm{TeV}$, y $\Gamma=10$ para el factor de Lorentz macroscópico del jet. Para la distribución de los protones relativistas mantuvimos los dos valores del índice espectral $\alpha=2$ y $\alpha=1.5$.

Los rayos $\gamma$ serán absorbidos por cascadas electromagnéticas en el material de la estrella, dejando quizás como rastro una línea de aniquilación de pares. Resulta más interesante analizar en este caso la producción de neutrinos que resultan del decaimiento de piones cargados. El material que proporciona las protones blanco sigue un perfil

$$
\rho(r)=\rho_{\mathrm{c}}\left[1-\left(r / R_{\star}\right)^{2}\right],
$$

con $\rho_{\mathrm{c}} \simeq 0.05 \mathrm{~g} / \mathrm{cm}^{3}$, de acuerdo con el tipo de estrella considerada.

\footnotetext{
${ }^{6}$ Las escalas temporales para la aceleración y difusión de las partículas son mucho menores que este valor: $t_{\mathrm{d}}\left(E_{p}^{\max }\right) \lesssim 29 \mathrm{~s} \mathrm{y} t_{\mathrm{d}}\left(E_{p}^{\min }\right) \lesssim 203 \mathrm{~s}$ para los modelos considerados.

${ }^{7} L_{\mathrm{j}}=q_{\mathrm{j}} 5.710^{38} \mathrm{erg} \mathrm{s}^{-1}$

${ }^{8}$ Se considera $\theta=0$, y cambia un signo por hacer la transformación de Lorentz al sistema de la estrella. Ver expresión (29) en Romero \& Orellana (2005).

${ }^{9} \mathrm{La}$ densidad del material donde se producen $\operatorname{los} \pi^{ \pm}$no es tan alta como para que los piones interactúen antes de decaer. La sección eficaz es $\sigma_{\pi^{ \pm} p} \sim 25 \mathrm{mb}$, y el tiempo de vida media $\tau_{\pi^{ \pm}} \sim 10^{-8} \mathrm{~s}$.
} 
En el sistema de referencia del observador los neutrinos alcanzan energías cercanas al $\mathrm{TeV}$, estando mayormente fuera del rango de observación de ICECUBE. Se obtiene $C_{\nu,(\alpha=2)}=3.710^{32} \mathrm{erg}^{-1} \mathrm{~s}^{-1}$ y $C_{\nu,(\alpha=1.5)}=3.110^{34} \mathrm{erg}^{-1} \mathrm{~s}^{-1}$. La señal para un MQ a unos $\sim 2 \mathrm{kpc}$ produce en este caso $N_{\mu}=3.25$ muones por año. A un nivel de $S / N=3$ es necesario entonces un tiempo de observación de $T_{\text {obs }} \sim 15$ años. Notese que es necesario que la línea de la visual forme un ángulo pequeño con el eje de los jets y a la vez con el plano de la órbita para garantizar la detección. 


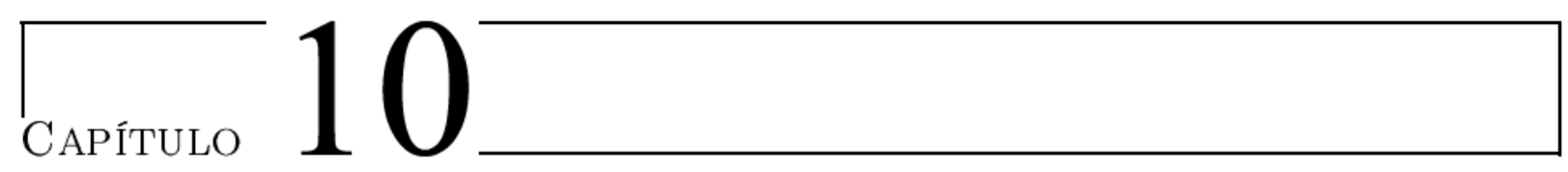

\section{Emisión gamma en pulsares acretantes}

Observaciones de líneas de ciclotrón en pulsares de rayos X han permitido estimar que algunas de las estrellas de neutrones allí presentes poseen campos magnéticos muy intensos, que llegan a ser del orden de varias veces $10^{12}$ Gauss. Los efectos que un campo magnético tan intenso pueda tener en la estructura del disco de acreción no pueden ser pasados por alto. En este capítulo se trata el caso de una HMXB donde el objeto compacto es un pulsar con campo magnético intenso. Presentamos primero el escenario, y luego el cálculo de la evolución de los parámetros del disco y de la emisión $\gamma$ durante una explosión mayor de rayos X. Como caso de estudio, se adopta el sistema A0535 +026. Los resultados han sido desarrollados en Orellana et al. (2007b) y siguen la línea de Orellana \& Romero (2005).

Para una estrella de neutrones acretante típica, el radio de Alfvén 1 suele ser $r_{\mathrm{A}} \sim 10^{3}$ $\mathrm{km}$, que es del orden de 100 veces el radio de la estrella. Este es un resultado importante en el caso de fuentes de rayos X luminosas. La dinámica del gas en la vecindad inmediata de la estrella de neutrones es dominada por el campo magnético.

La única forma en la que la materia puede ser acretada hasta la superficie de la estrella de neutrones, y liberar la máxima cantidad de energía potencial gravitatoria que fue convertida en cinética al caer, es llegando a la estrella por las zonas polares canalizada a través de las líneas de campo magnético. Así se forma una columna de acreción que se levanta del disco y conduce el material hacia la estrella a lo largo de las líneas del campo magnético.

Sólo si tal campo magnético es débil el gas podría caer directamente sobre la superficie de la estrella de neutrones, o al menos aproximarse lo suficiente como para que actuen mecanismos de evacuación como en un MQ (ver ej. Lipunov 1992; Massi \& KaufmanBernado 2007). Los detalles de la interacción entre el campo magnético y el plasma de un

\footnotetext{
${ }^{1}$ Definido como la distancia donde se igualan la presión del campo magnético y la presión ram del gas en caída libre hacia la estrella de neutrones. En términos de la tasa de acreción, el radio de Alfvén es$$
r_{\mathrm{A}}=B_{\mathrm{P}}^{4 / 7} R^{12 / 7}(2 G M)^{-1 / 7} \dot{M}_{\mathrm{ac}}^{-2 / 7} .
$$

donde $B_{\mathrm{p}}$ es el campo magnético en el polo de la estrella de neutrones, cuyo radio es $R$.
} 
disco de acreción son complejos pues dependen, entre otros factores, del ángulo entre el eje magnético de la estrella de neutrones y el eje de rotación del disco de acreción. Si bien el hecho de cómo el plasma en caída es canalizado por las líneas del campo magnético es un problema extremadamente difícil de resolver, se conoce que el campo magnético no penetra el plasma mucho más allá del radio de Alfvén2 La columna de acreción lleva corrientes inducidas en el plasma por el campo magnético y forma entonces corrientes superficiales que apantallan el campo fuera de $r_{\mathrm{A}}$ y lo refuerzan dentro. Por otro lado, la generación de corrientes inducidas en la superficie del disco modifica el valor del campo magnético en su interior. Esto se cuantifica con un factor de apantallamiento $\eta$, de modo que el campo magnético en el interior del disco ya no es el dipolar de la estrella de neutrones $\left(B_{\text {dipolar }}=-\mu / r^{3}\right.$, donde el valor del dipolo es $\mu=B_{\text {sup }} R^{3} / 2$ ). En su lugar, $B_{z}=\eta B_{\text {dipolar }}$, dentro del disco. El caso $\eta=1$ corresponde a un disco sin apantallamiento. Este factor $\eta$ depende de la difusividad magnética del plasma en el discd 3 y su valor determina la ubicación del borde interno del disco de acreción.

De acuerdo al modelo de disco estándar en torno de una estrella magnetizada (Gosh \& Lamb 1979) existe una amplia zona de transición entre las regiones exteriores del disco que no son perturbadas y la magnetósfera que corrota con la estrella de neutrones. En esa zona se produce la disrrupción del disco que resulta en el transporte de material por las líneas del campo magnético hasta la superficie estelar. Esta zona de transición puede dividirse en dos regiones: desde su radio exterior $R_{\text {ext }}$ hasta el radio interno donde deja de haber rotación Kepleriana $R_{o}$, y desde $R_{o}$ hasta el radio de corrotación $R_{\text {co }}$ donde el plasma es forzado a corrotar con la estrella. La velocidad angular cambia rapidamente de $\Omega_{d}$ en $R_{o}$ a $\Omega_{*}$ en $R_{\text {co }}$. La diferencia $R_{o}-R_{\text {co }}$ es mucho menor que $R_{o}$. En adelante llamaremos radio interno del disco al radio $R_{o}$.

Ha sido demostrado Wang (1996), que para plasma no totalmente diamagnético, el radio interno del disco se ubica en

$$
R_{o}=1.35 \gamma_{o}^{2 / 7} \eta^{4 / 7} r_{\mathrm{A}}
$$

donde el ángulo de inclinación magnética es $\gamma_{o}=-B_{\phi_{o}} / B_{z_{o}} \sim 1 \mathrm{y} r_{\mathrm{A}}$ es el radio de Alfvén para acreción esférica. El origen de la componente acimutal se relaciona con la rotación relativa. Para $r \gtrsim R_{o}$ las propiedades físicas del disco de acreción son las de un disco estándar. Aquí se han considerado las propiedades del disco cerca de $R_{o}$, suponiendo que en esta región la presión de radiación domina sobre las otras componentes (campo magnético y gas), y que el campo magnético está parcialmente apantallado, con $\eta=0.1$.

\footnotetext{
${ }^{2}$ Para encontrar el valor del radio hasta el cual el campo magnético de la estrella domina la situación dinámica, deben igualarse el torque ejercido por el disco de acreción sobre la estructura del campo magnético y el torque magnético asociado con el campo deformado a ese radio (ej, Longair 1994). Ese no resulta un cálculo trivial. Pero en general, se encuentra un resultado similar al obtenido en el caso de acreción esférica, dando un radio ligeramente menor al de Alfvén, tipicamente del orden de la mitad de éste.

${ }^{3}$ Algunos autores (ej. Gosh \& Lamb 1979) obtuvieron $\eta \simeq 0.2$, mediante el cálculo de las corrientes toroidales inducidas por el flujo radial en $R_{o}$. Otros autores han argumentado que el campo atraviesa por completo el disco y $\eta \simeq 1$ (ver Li 1997, Wang 1996 y sus referencias).
} 


\subsection{Aceleración de partículas en la magnetósfera}

Como se ha visto, las interacciones electrodinámicas de una estrella de neutrones magnetizada con el disco de acreción que la rodea son en principio muy complejas. En un planteo completo aparecen inestabilidades magnetohidrodinámicas y reconexión de líneas de campo magnético. También podrían existir campos electricos radiales y varios efectos relacionados.

Cheng \& Ruderman $(1989,1991)$ han considerado la estructura del campo eléctrico y la distribución de plasma en un modelo simplificado del dínamo que forma la estrella de neutrones con su disco de acreción. Se asume simetría axial y que el disco es un plasma perfectamente conductor ( $\vec{E} \cdot \vec{B}=0$ en su superficie). La densidad de carga $\rho$ se anula en la superficie $\vec{\Omega} \cdot \vec{B}=0$ y las cargas en la magnetósfera quedan separadas en dos regiones 4 . Si se consideran los efectos inerciales, la fuerza que realmente actúa sobre las partículas del plasma tiene una componente a lo largo de la línea de campo magnético que separa las partículas según el signo de la carga.

Es de particular interés el caso de un disco de acreción Kepleriano en rotación alineada con la estrella de neutrones, y cuyo radio interno $R_{o}$ rota más rápido que la estrella. Un planteo similar al anterior lleva a que la magnetósfera está separada a lo largo de la superficie nula $\vec{\Omega} \cdot \vec{B}=0$. La magnetósfera queda de hecho dividida en tres partes (ver Figura 10.1):

i) Una parte acoplada con la estrella por las líneas de campo magnético que corrotan con ella.

ii) Una parte vinculada al disco por líneas de campo magnético que no cruzan la superficie nula entre la estrella y el disco. Esta parte corrota con el disco y sigue valiendo $\vec{E} \cdot \vec{B}=0$.

iii) En el entorno de la superficie nula, entre las regiones i) y ii) queda una brecha vacía de plasma. En esta zona $\vec{E} \cdot \vec{B} \neq 0$.

\footnotetext{
${ }^{4}$ Para comprender la situación primero se puede plantear el caso de rotación rígida, donde se igualan la velocidad angular del disco $\left(\vec{\Omega}_{\mathrm{d}}\right)$ y de la estrella $\left(\vec{\Omega}_{*}\right): \vec{\Omega}_{\mathrm{d}}=\vec{\Omega}_{*}=\vec{\Omega}$. Para un estado estacionario (de equilibrio) la fuerza resultante sobre cada partícula se anula. Si se desprecian los efectos inerciales, es la fuerza de Lorentz la que debe ser nula. Entonces, dentro del cilindro de luz que se define igual que para un pulsar aislado, se tiene

$$
\vec{E}=\left(\frac{\vec{\Omega} \times \vec{r}}{c}\right) \times \vec{B}
$$
}

de donde Cheng \& Ruderman (1991) obtienen:

$$
\rho=\frac{\vec{\nabla} \cdot \vec{E}}{4 \pi}=\frac{\vec{\Omega} \cdot \vec{B}}{2 \pi c}\left[1-\left(\frac{\vec{\Omega} \times \vec{r}}{c}\right)^{2}\right]^{-1} .
$$




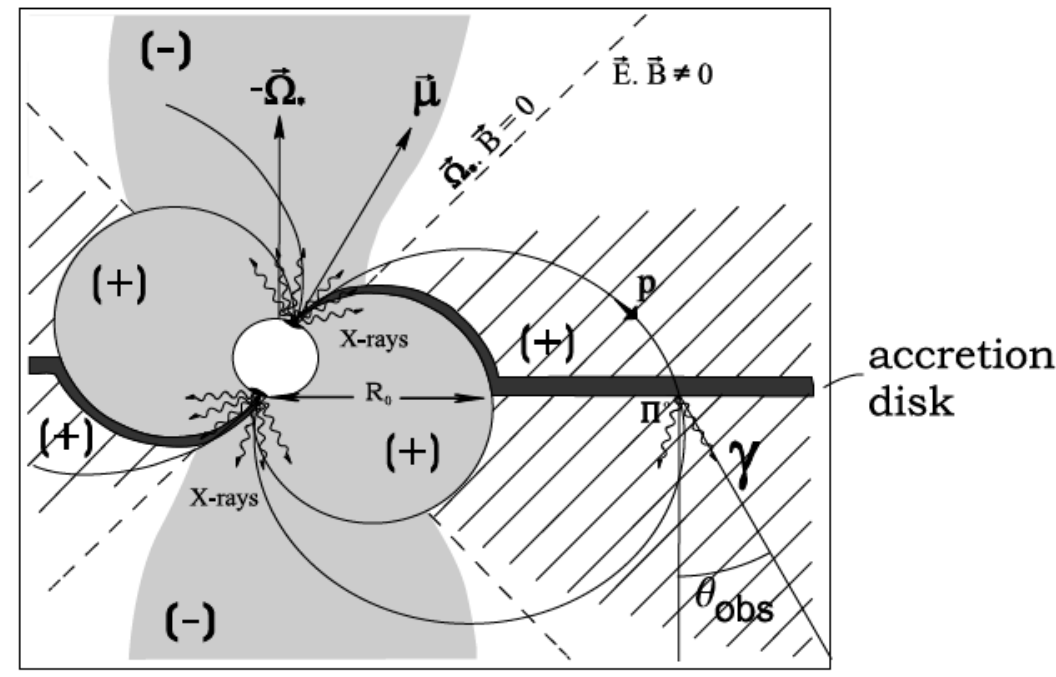

Figura 10.1: Las tres regiones de la magnetósfera cuando $\Omega_{d}>\Omega_{*}$. La región en gris corrota con la estrella, La región rayada corrota con el disco. Entre ellas existe una región que se encuentra vacía de plasma, y allí es $\vec{E} . \vec{B} \neq 0$. Esquema adaptado de Romero et al. (2001).

En esta geometría con $\Omega_{\mathrm{d}}>\Omega_{*}$ y una brecha en el plasma, $\vec{B}$ es independiente del tiempo, luego $\oint \vec{E} . d \vec{s}=0$. Eligiendo un camino de integración adecuado, puede calcularse la fuerza electromotriz $\varepsilon$ que aparece entre las superficies que delimitan la brecha y con un cambio de signo, se establece de una diferencia de potencial (electrostático). La diferencia de potencial en la brecha puede alcanzar un valor estático, que en condiciones de apantallamiento fuerte es

$$
V_{\max } \approx 4 \times 10^{14} \beta^{-5 / 2} M^{1 / 7} R_{6}^{-4 / 7} L_{37}^{5 / 7} B_{12}^{-3 / 7} V
$$

donde $M$ es la masa de la estrella de neutrones en masas solares, $R_{6} \equiv R / 10^{6} \mathrm{~cm}$ es su radio, $B_{12} \equiv B_{\mathrm{p}} / 10^{12} \mathrm{G}$ su campo magnético, $L_{37}$ la luminosidad de rayos X (del disco de acreción) en unidades de $10^{37} \mathrm{erg} \mathrm{s}^{-1}$, y $\beta \equiv 2 R_{o} / r_{\mathrm{A}}$ es dos veces el cociente entre el radio interno del disco de acreción y el radio de Alfvén. Para un pulsar de rayos X con parámetros típicos se tiene $V_{\max } \sim 10^{13-14} \mathrm{~V}$ (Romero et al. 2001).

Los protones que entran a la brecha, provenientes de la zona que corrota con la estrella son acelerados hasta energías $E_{p} \sim e V_{\max }$. Entonces, para $V_{\max } \gtrsim 10^{13} \mathrm{~V}$ se tiene una corriente de protones ultrarrelativistas, pues $E_{p} \gg m_{p} c^{2}$. Estos protones son dirigidos hacia el disco de acreción siguiendo las líneas del campo magnético, y sufren a lo largo de esta trayectoria pérdidas energéticas (por radiación de curvatura y foto-producción de hadrones) muy poco relevantes, pues $\Delta E / E \ll 1$. La corriente máxima que fluye atravesando la brecha queda determinada por la condición de que el campo magnético acimutal, inducido

\footnotetext{
${ }^{5}$ Justo fuera de la superficie de la estrella de neutrones la fuerza de Lorentz sobre las partículas cargadas excede enormemente la fuerza de atracción gravitacional (Goldreich \& Julian 1969). El tema del viento de un pulsar se comentó en la Sección 3.2
} 
por la corriente, no exceda el valor inicial del campo magnético. Luego

$$
J_{\max } \approx 1.5 \times 10^{24} \beta^{-2} M^{-2 / 7} R_{6}^{1 / 7} L_{37}^{4 / 7} B_{12}^{-1 / 7} \text { esu s }^{-1} .
$$

Esto hace que la corriente de protones que impacta en el disco sea tremendamente energética, siendo la potencia total depositada en el disco $\dot{E}_{\text {iny }} \sim J_{\max } V_{\max } \sim 10^{36} \mathrm{erg} \mathrm{s}^{-1}$. Notese que el impacto de protones relativistas sobre el disco no sólo ocurre en un punto específico, sino sobre un área con una cierta extensión radial, ya que la base de las líneas de campo es arrastrada con el material acretado. Algunos autores incorporan un factor de colimación o beaming $(\Delta \Omega / 4 \pi \approx 0.3)$ a la luminosidad que escapa, para tener en cuenta en forma fenomenológica la direccionalidad de la emisión que emerge (Cheng et al. 1991, Romero et al. 2001).

Otro efecto que genera el bombardeo del disco por esta corriente de protones es la perturbación de su estructura. En la actualidad las inestabilidades en discos de acreción se tratan mediante simulaciones numéricas (ej. Teresi et al. 2004, Merloni \& Nayakshin 2006). Estos autores señalan que el efecto estabilizante de la advección en dirección radial probablemente lleve a un comportamiento límite de tipo oscilatorio (probablemente dando origen a los rasgos de oscilaciones cuasi-periódicas que se observan en rayos X). En el caso en que la perturbación que produce la corriente de protones no pueda confinarse a una oscilación suave de la altura del disco, el crecimiento exponencial del espesor llevaría a la eliminación de la perturbación por el llenado de la brecha de aceleración. En tal caso, el disco se reacomodaría a su estructura normal hasta que eventualmente se reestablezca la corriente de protones, resultando también un comportamiento oscilatorio (Orellana et al. 2007b).

\subsubsection{Producción de rayos gamma y procesamiento dentro del disco de acreción}

La colisión de protones relativistas contra el disco de acreción produce los resultados usuales de interacciones $p p$. Aquí se estudió en detalle la radiación $\gamma$ que se produce por decaimiento de piones neutros, y los efectos de su propagación. A tal fin se consideró como caso de estudio la binaria de rayos X A $0535+26$. Este sistema presenta un comportamiento multi-frecuencia notable, por lo que ha sido estudiado en forma intensiva mediante diversas campañas de observación. Giovannelli \& Graziati (1992) brindan un artículo de revisión sobre esta binarial 6 .

A $0535+26$ es un sistema con alta excentricidad, formado por un pulsar de rayos X (con período $104 \mathrm{~s}$ ), y la estrella HDE 245770 de tipo espectral B0III. El período orbital es de 111 días. La distancia estimada es de $2.6 \pm 0.4 \mathrm{kpc}$. Frecuentemente se han observado eventos transitorios de tipo explosivo en la emisión de rayos X de esta fuente. Dichos flares y outbursts han mostrado diversas intensidades (ver Coe et al. 2006 y sus referencias).

\footnotetext{
${ }^{6}$ Aunque este review ya empieza a quedar desactualizado, en vista de las nuevas observaciones.
} 
Durante etapas de actividad en rayos $\mathrm{X}$ se han observado transiciones entre estados de emisión en el IR, lo cual parece sugerir que las explosiones en rayos X se vinculan a un incremento en la tasa de acreción de masa, y a la presencia de un disco transitorio alrededor de la estrella Be (Haigh et al. 2004). En Grundstrom et al. (2007b) se reportan variaciones de la línea $\mathrm{H}_{\alpha}$.

Ciertos rasgos en el espectro, cerca de los $110 \mathrm{KeV}$, han sido observados en A0535+26. Éstos se relacionan con emisión sincrotrón, e indican que el campo magnético dipolar de la estrella de neutrones es $\sim 10^{13}$ Gauss (Finger et al. 1996). La propuesta de que el mecanismo de Cheng-Ruderman podría estar operando en $\mathrm{A} 0535+26$ fue realizada por Romero et al. (2001) para brindar una explicación que vincule a este sistema con la fuente de rayos $\gamma$ $3 \mathrm{EG} \mathrm{J} 0542+2610$, con la que coincide posicionalmnente. En Orellana et al. (2007b) hemos profundizado en el tratamiento cuantitativo de aquella propuesta original. Consideramos una explosión gigante en rayos X que ha efectuado A0535 + 26 durante Febrero-Marzo de 1994] Se ajustó una función suave del tiempo a la curva de luz (dada en la Figura 9 de Finger et al. 1996), que determina $\dot{M}_{\text {ac }}(t)$ cuando se conoce la eficiencia radiativa, $\epsilon$. Aquí hemos adoptado $\epsilon=0.1$, y supusimos que la luminosidad del disco de acreción alcanza $L \sim 10^{38} \mathrm{erg} \mathrm{s}^{-1}$ en el máximo de la explosión 8 . Se centró el máximo de la curva a tiempo cero, con lo cual el parámetro $t$ varía entre los días -14 y 28.

Una aproximación de primer orden para poder evaluar los efectos del impacto de los protones relativistas contra el disco (incluyendo la emisión gamma) es suponer que todos (los protones) llegan al disco con la misma energía $E_{p} \sim e V_{\max }$, con $V_{\max }$ dado por (10.2). Luego el flujo de protones $J_{p}$ es una función delta de Dirac en la variable energía, y puede ser estimado como

$$
J_{p}(E) \sim \frac{J_{\max }}{e \pi R_{0}^{2}} \delta\left(E-E_{p}\right) \quad \frac{\text { protones }}{\mathrm{s} \mathrm{cm}^{2}} .
$$

Para calcular la emisividad de los fotones $\gamma$ se aplicó la aproximación de la función $\delta$ para la sección eficaz diferencial (Aharonian \& Atoyan 2000). Dada la expresión (10.4) se obtiene

$$
q_{\gamma}\left(E_{\gamma}\right) \approx \frac{2 J_{\max }}{e \pi R_{0}^{2}} \frac{\sigma_{p p}\left(E_{p}\right)}{\sqrt{\kappa^{2}\left(E_{p}-m_{p} c^{2}\right)^{2}-m_{\pi}^{2} c^{4}}} \frac{\text { fotones }}{\text { átomo } \mathrm{cm}^{3} \mathrm{eVs}},
$$

para $E_{\gamma}$ entre $E_{1}=0.5 E_{\pi}\left(1-v_{\pi} / c\right)$ y $E_{2}=0.5 E_{\pi}\left(1+v_{\pi} / c\right)$, mientras que $q_{\gamma}\left(E_{\gamma}\right)=0$ fuera de ese rango. En estas expresiones $v_{\pi}$ es la velocidad de un pión neutro con energía $E_{\pi}=\kappa\left(E_{p}-m_{p} c^{2}\right), \mathrm{y} \kappa \sim 0.17$ es la fracción de la energía cinética del protón que se lleva un $\pi^{0}$ "lider" por colisión (ver Apéndice).

La luminosidad que se produce en fotones $\gamma$ resulta

$$
L^{0}\left(E_{\gamma}\right)=\pi R_{0}^{2} \int_{0}^{2 h} q_{\gamma}\left(E_{\gamma}\right) E_{\gamma}{ }^{2} n_{p} e^{-z / \lambda_{p p}} d z \quad \Rightarrow L^{0}\left(E_{\gamma}\right) \propto E_{\gamma}{ }^{2},
$$

\footnotetext{
${ }^{7}$ La fuente se observó luego inactiva hasta Mayo de 2005 (Coe et al. 2006), con el período del pulsar en constante aumento durante esos 10 años (Hill et al. 2007).

${ }^{8}$ Esta parece ser una extrapolación razonable, en base a los datos disponibles que corresponden a la emisión de rayos X duros (20-100 keV observados con el instrumento BATSE) que se originan en la zona polar de la estrella de neutrones por el impacto de la columna de acreción.
} 
donde $n_{p}$ es la densidad numérica de protones en el disco, y $\lambda_{p p}$ el camino libre medio para un protón que impacta en el mismo. Nótese que los fotones con energía $E_{\gamma}^{\max } \approx \kappa E_{p}$ dominan este espectro "invertido". Teniendo en cuenta la profundidad óptica del material del disco que un fotón debe atravesar puede estimarse la luminosidad que escapa, $L_{\mathrm{esc}}^{0}=$ $L^{0} e^{-\tau_{\gamma p}}$.

Los fotones que escapan del disco siguen la dirección original de movimiento del protón. Los que son absorbidos inician cascadas electromagnéticas dentro del disco. No estando por completo específicada la dirección del campo magnético 10 , hemos considerado trayectorias que forman un ángulo de $45^{\circ}$ con el eje $z$ (pues $B_{\phi} \sim B_{z}$ ). Entre los procesos de pérdida de energía que los electrones y positrones relativistas (generados en las absorciones $\gamma p$ ) sufren al moverse dentro del disco, hemos hallado que domina el proceso de radiación de frenado o Bremsstrahlung. Introduciendo algunas simplificaciones, las cascadas electromagnéticas dominadas por este proceso pueden ser tratadas en forma analítica, mediante la resolución de las correspondientes ecuaciones de transporte. Implementamos las soluciones para el espectro diferencial promedid 11 dadas por Rossi \& Greisen (1941), en el marco de la llamada "Aproximación A", para dos instancias representativas de las diferentes condiciones que se dan en el disco de acreción durante la explosión. Esto es, un momento cercano al comienzo de la explosión, $t=-11$ días, y en el máximo, $t=0$.

Los fotones que dan inicio a las cascadas dentro del disco tienen energías $E_{0}=E_{\gamma}^{\max }(t=$ $0) \sim 830 \mathrm{TeV}$ y $E_{0} \sim 215 \mathrm{TeV}$ para $t=-11$ días. La unidad de espesor al tratar con estas cascadas es la llamada "longitud de radiación" (radiation length), $X_{0}$, dada por

$$
\frac{1}{X_{0}}=n\left(2.318 Z(Z+1) \frac{\ln \left(183 Z^{-1 / 3}\right)}{1+0.12(Z / 82)^{2}} \mathrm{mb}\right)
$$

donde $Z$ es la carga de los núcleos que hacen de blanco y $n$ su densidad numérica. La cascada atraviesa un medio más grueso en $t=0$ que al comienzo de la explosión (ver en la Figura 10.3 la evolución de $\tau_{\gamma p}$ ). Esta es la causa de las diferencias que pueden verse en los espectros resultantes que se muestran en la Figura 10.4 Obtuvimos $L_{\mathrm{A}} \sim$ $10^{28}(E / \mathrm{GeV})^{-0.6} \mathrm{erg} \mathrm{s}^{-1}$ para $t=-11$ días, que contribuye a la emisión que sale del disco $L_{\text {esc }}^{0} \sim 2 \times 10^{23}(E / \mathrm{GeV})^{2} \mathrm{erg} \mathrm{s}^{-1}$. El término $L_{\mathrm{A}}$ domina la emisión por debajo de los $\sim 40$ $\mathrm{GeV}$. La radiación saliente $L_{\mathrm{A}}+L_{\text {esc }}^{0}$ en $t=0$ resulta en niveles muy bajos de flujo como para ser detectable por los instrumentos actuales. Por otro lado, la emisión $\gamma$ de muy alta energía que emerge del disco al comienzo de la explosión inicia cascadas electromagnéticas en el campo radiativo de la estrella de gran masa, similares a las discutidas en el Capítulo 6.

\footnotetext{
${ }^{9}$ Donde el proceso de absorción relevante es la interacción con el campo Coulombiano de los núcleos (para crear pares $e^{ \pm}$). La sección eficaz de este proceso es $\sigma_{\gamma p} \sim 10^{-26} \mathrm{~cm}$.

${ }^{10} \mathrm{La}$ componente radial del campo magnético que determina $\theta_{\text {obs }}$ queda aquí libre; es función del ángulo que forman $\bar{\Omega}$ y $\bar{\mu}$, y es modificada por el arrastre que tenga el material acretado.

${ }^{11}$ Específicamente, en el marco de esta teoría a energías $E \gg m_{e} c^{2} Z^{-1 / 3} / \alpha$ se desprecian tanto las colisiones como el proceso Compton, y se utilizan las expresiones asintóticas para describir los procesos de radiación y creación de pares.
} 

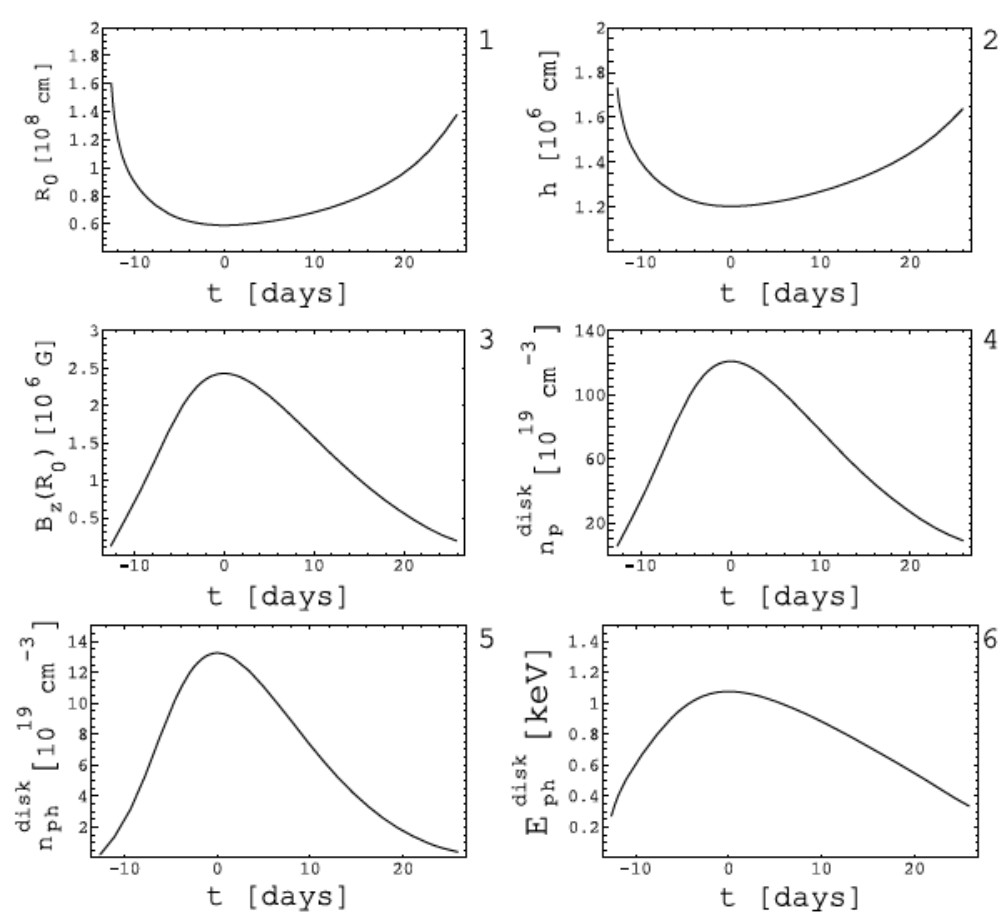

Figura 10.2: Evolución temporal de los parámetros del disco de acreción. El máximo de la explosión en rayos $\mathrm{X}$ coincide con $t=0$.

1) Radio interno $R_{o}$ donde se levanta la columna de acreción. Las siguientes cantidades son valores estimados en $R_{o}$. 2) Semialtura del disco. 3) Componente vertical del campo magnético para el factor de apantallamiento $\eta=0.1$. 4) Densidad numérica de protones en el disco 5) Densidad numérica de fotones térmicos emitidos por el material acretado. 6) Energía característica de dichos fotones.
Figura 10.3: Parámetros asociados con los efectos del impacto de la corriente de protones relativistas contra del disco de acreción, y la luminosidad resultante por decaimiento de piones neutros.

1) Potencia inyectada en el disco por el bombardeo de protones. 2) Energía máxima de los rayos $\gamma$. 3) Profundidad óptica para los protones que ingresan al disco $\left(\tau_{p p}=2 h / \lambda_{p p}\right)$. 4) Luminosidad generada a través de decaimientos $\pi^{0} \rightarrow 2 \gamma$. 5) Profundidad óptica para los fotones más energéticos. 6) Luminosidad gamma que escapa, estimada co$\operatorname{mo} L^{0} e^{-\tau_{\gamma p}}$.
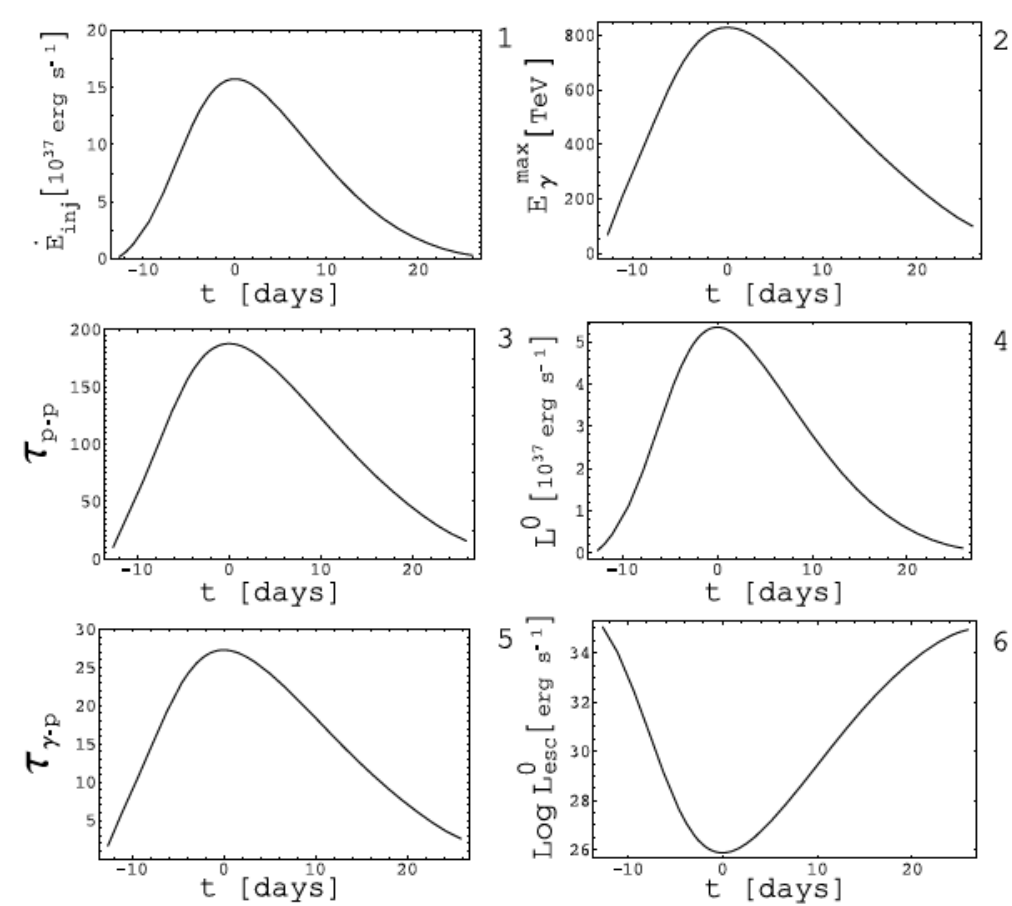


\subsubsection{Procesamiento en la fotósfera}

La opacidad a la propagación de los rayos $\gamma$ que emergen del disco resulta en realidad de una combinación de factores. A energías de algunos GeV los rayos X dominan la absorción, y en esta ventana del espectro se tiene la emisión del propio disco y la que resulta del choque de la columna de acreción contra la superficie de la estrella de neutrones. A energías mayores, es el campo radiativo estelar el que domina la absorción, si bien otra componente de fotones IR-ópticos puede ser aportada por el viento estelar.

Aquí consideramos solamente las cascadas unidimensionales que se desarrollan en el campo radiativo de la estrella. En este caso la temperatura de la estrella es $T_{\star}=3 \times 10^{4}$ $\mathrm{K}$, y su radio $R_{\star}=10^{12} \mathrm{~cm}$. La separación a la estrella de neutrones cerca del comienzo de la explosión en rayos $\mathrm{X}$ es $a \simeq 10^{13} \mathrm{~cm}$. Sólo consideramos esta configuración ya que en el máximo de la explosión es poco significativa la emisión $\gamma$ que emerge del disco. El índice espectral de los fotones que inician estas cascadas es $\alpha=0$. Los resultados de las simulaciones Monte Carlo de las cascadas electromagnéticas que se desarrollan en este medio se muestran en la Figura 10.5. Se varió el ángulo $\theta_{\text {obs, }}$ para investigar la dependencia con el ángulo de propagación. Aquí $\theta_{\text {obs }}$ se mide en el plano que contiene la estrella de neutrones y la estrella masiva, desde el eje $z$, en dirección de la estrella (ver el esquema 10.1). La ligera dependencia $\operatorname{con} \theta_{\text {obs }}$ que se encuentra esta en acuerdo con lo esperado; para un ángulo mayor, y una trayectoria más próxima a la estrella el efecto sobre el espectro es más marcado. A energías gamma del orden de centenares de $\mathrm{TeV}$ el espectro no se ve afectado, por lo que la potencia detectable es todavía dominada por los fotones con energía $\sim E_{0}$.

El efecto de una inyección monoenergética de protones en el disco de acreción es la producción final de emisión gamma con un espectro de índice $\sim-2.2$ entre los $\sim 10 \mathrm{GeV}$ y
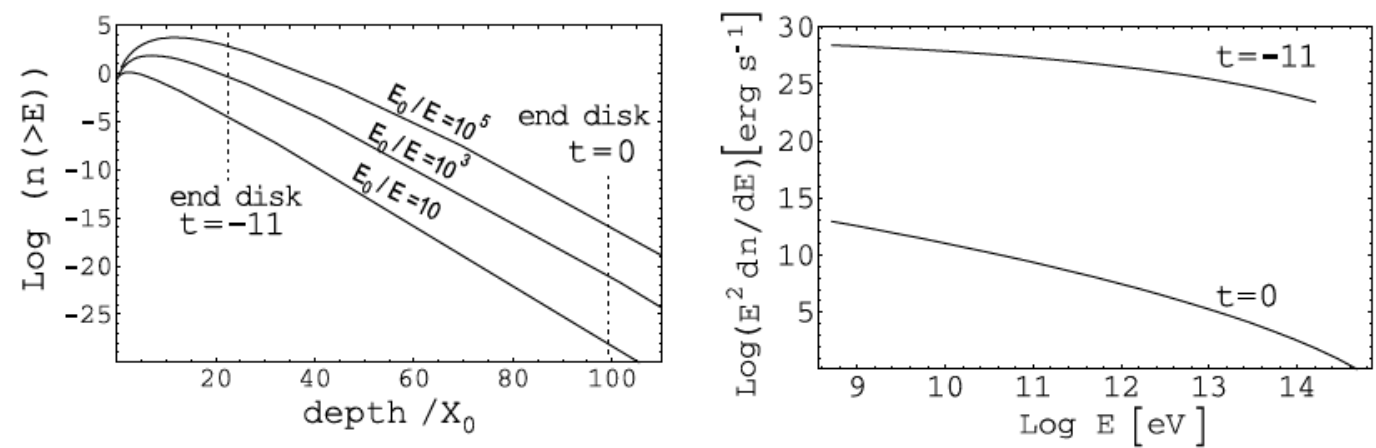

Figura 10.4: Comportamiento promedio de las cascadas electromagnéticas que se desarrollan en el disco de acreción, iniciadas por fotones con energía $E_{0}$. A la izquierda: número de fotones con energía mayor que $E$ en la cascada iniciada por un sólo fotón de energía $E_{0}$. El espesor del disco varía con el tiempo. A la derecha: luminosidad que emerge del disco luego del reprocesamiento por cascadas. Las energías iniciales son las de los fotones que dominan el espectro en cada caso $\left(E_{0}=215 \mathrm{TeV}\right.$ para $t=-11$ y $E_{0}=828 \mathrm{TeV}$ para $\left.t=0\right)$. 


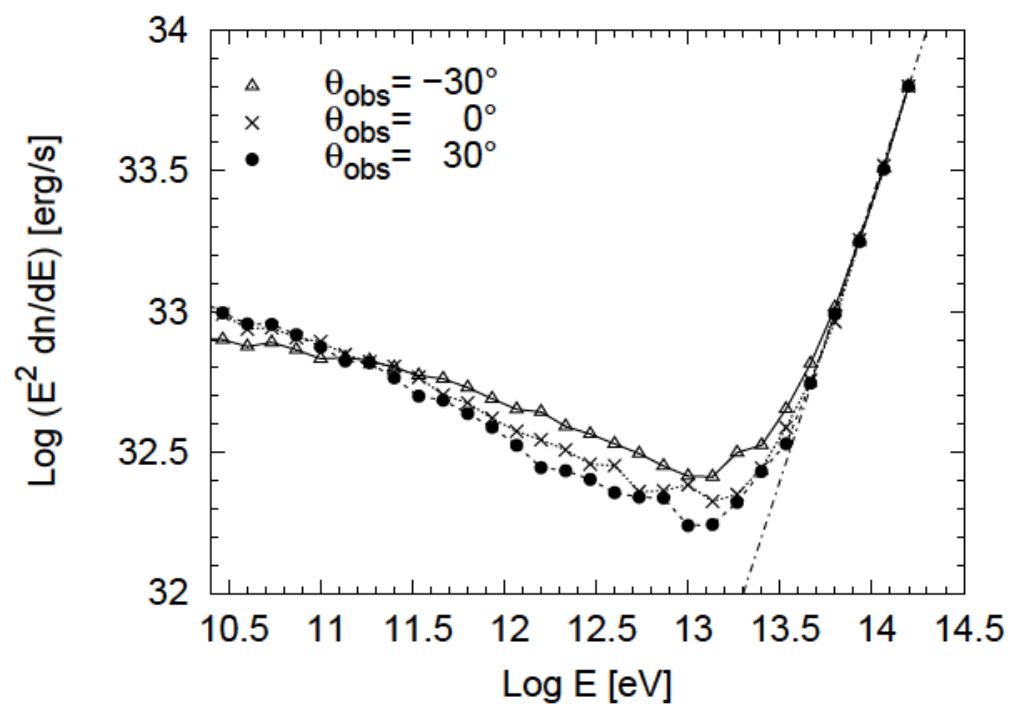

Figura 10.5: Luminosidad resultante del reprocesamiento por cascadas electromagnéticas en el campo radiativo de la estrella. La línea indica la pendiente del espectro inyectado.

$\sim 10 \mathrm{TeV}$. Las luminosidades que se obtienen rondan los $6 \times 10^{32} \mathrm{erg} \mathrm{s}^{-1}$ para $E_{\gamma} \sim 100 \mathrm{GeV}$. Las características de espectros como los obtenidos pueden ser detectadas por telescopios Cherenkov si se logran implementar observaciones coordinadas con los monitoreos en rayos $\mathrm{X}$ que den aviso del comienzo de una erupción. Se esperaría entonces, en base a nuestros resultados, que se observe una anticorrelación entre la señal en rayos X y en rayos $\gamma$. 
$\prod_{\text {CAPÍTULO }} 11$

\section{Conclusiones y perspectivas}

A lo largo de la investigación vinculada a esta tesis se estudiaron modelos de binarias de rayos $\gamma$ con acreción. Este tema ha experimentado un progreso radical durante los últimos años debido a los resultados obtenidos por telescopios Cherenkov. Gracias a ellos, al momento de escribir estas líneas se conocen 4 binarias de rayos $\gamma$, cuya emisión varía a energías mayores que $\sim 200 \mathrm{GeV}$.

Los modelos estudiados en esta tesis se enfocan en dos sub-clases de HMXB:

- Microcuásares, caracterizados por la presencia de jets. Cabe destacar que ha llegado a aceptarse que el proceso de formación de los jets está asociado al proceso de acreción, de forma que ambos fenómenos son simbióticos y se presentan en una gran variedad de escenarios astrofísicos, desde objetos estelares jóvenes a núcleos activos de galaxias.

- Binarias que contienen una estrella de neutrones con campo magnético lo suficientemente intenso como para frenar la caída del material acretado a una distancia del orden del radio de Alfvén. Estos sistemas pueden desarrollar regiones de aceleración de partículas tipo capacitor en su magnetósfera.

Estos escenarios son muy complejos y no pretendemos haber dado cuenta de la cantidad de detalles físicos involucrados, cuya riqueza recién comienza a ser explorada en trabajos teóricos contemporáneos. Si acaso, hemos indagado en la superficie, y bajo hipótesis simplificadoras.

El principal mecanismo de emisión gamma que hemos considerado es la interacción de protones relativistas con el medio local. Recientemente incluso la descripción de este proceso ha visto avances que tratamos de incorporar en nuestro trabajo (como las parametrizaciones de secciones eficaces y emisividades dadas por Kelner et al. 2006).

$\mathrm{Al}$ suponer un contenido de protones relativistas en el jet de un MQ de gran masa, las interacciones con protones fríos que a modo de blanco provee el viento estelar, dan lugar a 
una emisión intensa a energías $E_{\gamma} \gtrsim 1 \mathrm{GeV}$, como fue sugerido por Romero et al. (2003). Es de destacar, que hasta ahora, sólo binarias con estrellas primarias calientes y de gran masa han sido detectadas a muy altas energías.

Hemos refinado el modelo de MQ hadrónico para aplicarlo al caso concreto de la fuente LS I +61 303. Los resultados obtenidos son consistentes con las restricciones impuestas a la emisión gamma de esta fuente por el telescopio MAGIC. Se utilizaron aquí estimaciones de la tasa de acreción de masa en un régimen de Bondi-Hoyle. Sin embargo, los resultados de simulaciones dinámicas SPH, que hemos implementado luego en colaboración con el Dr. Atsuo Okazaki, sugieren que el proceso de transferencia de masa no es adecuadamente descripto por la hipótesis de acreción esférica. De hecho, aún en base a estas simulaciones, por cuestiones de resolución computacional, resulta dudoso que el valor de $\dot{M}_{\text {ac }}$ alcance un máximo local durante el pasaje por el periastro.

A su vez, simulaciones planteando en el sistema la colisión de vientos pulsar/estrella revelan que este escenario, alternativo al de MQ, no puede dar cuenta de la morfología detectada en radio, por lo que se debilitan los argumentos sugeridos en su favor. Este escenario también adolece de graves problemas a la hora de reproducir las curvas de luz observadas.

Hemos investigado la radiación emitida por leptones secundarios en un modelo de MQ hadrónico, considerando una aplicación a un sistema similar a Cyg X-1 (con viento esférico). Calculamos la emisión sincrotrón y Compton inversa de los leptones generados dentro del jet por decaimiento de piones cargados (con pérdidas de energía calculadas en forma consistente, pero sin mecanismos de reaceleración). En este marco resultó dominante la emisión a energías mayores que $\sim 1 \mathrm{MeV}$, proveniente del reprocesamiento por cascadas electromagnéticas que se desarrollan en el campo radiativo de la estrella donante de masa.

Para estimar los resultados de tales cascadas hemos desarrollado un código de simulación Monte Carlo que tiene en cuenta la anisotropía y peculiaridades del campo radiativo absorbente, en la evolución de una cascada unidimensional. El efecto de redistribución de la energía en el espectro ha quedado bien ilustrado en nuestros resultados, y hace pensar que puede ser importante mejorar el código desarrollado incorporando los efectos de la presencia de un campo magnético. Si éste es más bien ordenado, han de calcularse las trayectorias de las partículas individuales. Si al contrario, es predominante la componente randómica, podría adoptarse una hipótesis de isotropización local (en el lugar de creación de cada par), como en los trabajos de Bednarek y colaboradores. En esta dirección se encaminan los próximos pasos del presente programa de investigación.

Volviendo a los escenarios contemplados, en el caso de un MQ con inclinación extrema entre el jet y el plano orbital estudiamos la emisión que resulta de interacciones entre protones de la base del viento y protones reacelerados en el frente de choque jet-viento. Algunos de los modelos calculados preveen una luminosidad de varias veces $10^{32} \mathrm{erg} \mathrm{s}^{-1}$, siendo fuentes variables que en caso de no encontrarse excesivamente lejanas serían detectables por los nuevos telescopios Cherenkov. Sólo en el caso de un jet con alto contenido en hadrones relativistas, que interactúa directamente con la estrella, se obtiene una emisión 
importante de neutrinos.

Para estudiar el caso de un pulsar acretante se consideró una explosión de rayos X del sistema A0535+262. Calculamos la emisión gamma, encontrando que los procesos que ocurren dentro del disco absorben la emisión durante el máximo de dicha explosión. Ya sea al comienzo o al final de la misma, la radiación que puede escapar del disco es reprocesada en la fotósfera de la estrella y resulta, según nuestras simulaciones de desarrollo de cascadas, con un espectro suave $(\alpha \sim-2.2)$ hasta energías de $\sim 100 \mathrm{GeV}$, y muy duro $(\alpha=0)$ a energías mayores. Se predice la detección de pulsares acretantes como fuentes $\gamma$ transitorias, con una anticorrelación entre la radiación X y la $\gamma$.

Los próximos resultados observacionales, en particular los que aporte GLAST con su excelente resolución y sensibilidad, serán muy útiles para testear las predicciones de modelos como los que se han desarrollado aquí y ayudarán a mejorar las hipótesis adoptadas. A su vez, el importante desarrollo que está teniendo la astronomía de rayos $\gamma$ seguramente abrirá en un futuro inmediato nuevos interrogantes vinculados a la radiación de binarias con acreción. Esto brindará nuevas y excitantes oportunidades de continuar avanzando en nuestro conocimiento de los microcuásares y sistemas similares. 


\section{Bibliografía}

[1] Abdo, A.A. et al. 2007, ApJ 664, L91

[2] Aharonian, F.A. \& Atoyan A. M. 1981, Astr. Space Sci. 79, 321

[3] Aharonian, F.A. \& Atoyan A. M. 1996, A\&A 309, 917

[4] Aharonian, F.A. \& Atoyan, A.M. 2000, A\&A 362, 937

[5] Aharonian, F.A. \& Bogovalov, S.V. 2003, New Astronomy 8, 85

[6] Aharonian, F.A., Very High Energy Cosmic Gamma-Ray Radiation, World Scientific Publishing, 2004.

[7] Aharonian, F.A. \& Plyasheshnikov, A.V. 2003, Astroparticle Physics 19, 525

[8] Aharonian, F.A. et al. (HESS coll.) 2005a, Science 309, 746

[9] Aharonian, F.A., et al. (HESS coll.) 2005b, A\&A 442, 1

[10] Aharonian, F.A., Anchordoqui, L.A., Khangulyan, D., Montaruli, T. 2006, Journal of Physics: Conference Series 39, 408

[11] Aharonian F.A., et al. (HESS coll.) 2007a, Phys. Rev. D75, 042004

[12] Aharonian F.A., et al. (HESS coll.) 2007b, A\&A 167, 1075

[13] Albert, J. et al. (MAGIC coll.) 2006, Science 312, 1771

[14] Albert, J. et al. (MAGIC coll.) 2007, ApJ 665, L51

[15] Alvarez-Muñiz, J. \& Halzen, F. 2002, ApJ 576, L33

[16] Anchordoqui, L.A., Torres, D.F., McCauley, T., et al. 2003, ApJ 589, 481

[17] Anguelov, V. \& Vankov, H. 1999, J. Phys. G: Nuc. Part. Phys. 25, 1755

[18] Antokhin, I.I., Owocki, S.P. \& Brown, J.C. 2004, ApJ 611, 434

[19] Atoyan, A.M. \& Aharonian, F.A. 1999, MNRAS 302, 253

[20] Band, D.L. \& Grindlay, J.E. 1986, ApJ 311, 595 
[21] Bardeen, J.M. \& Petterson, J.A. 1975, ApJ 195, L65

[22] Baring, M.G., et al. 2005, Adv. Space Res. 35, 1041.

[23] Becker, P.A. \& Kafatos, M. 1995, ApJ 453, 83

[24] Bednarek, W. 1997, A\&A 322, 523

[25] Bednarek, W. 2000, A\&A 362,646

[26] Bednarek, W. 2005, MNRAS 363, L46

[27] Bednarek, W. \& Protheroe, R. J. 1997, MNRAS 287, L9

[28] Begelman, M.C., King, A.R. \& Pringle, J.E. 1980, MNRAS 370, 399

[29] Benaglia P. \& Cappa C.E. 1999, A\&A 346, 979

[30] Benaglia P., Romero G.E., Stevens I., Torres D.F. 2001, A\&A 366, 605

[31] Benaglia P. \& Romero G.E. 2003, A\&A 399, 1121

[32] Biermann, P. L. \& Strittmatter, P. A. 1987, ApJ 322, 643

[33] Bignami, G.F. 1980, IAU Circ No 3518

[34] Blandford, R.D. \& Payne, D.G. 1982, MNRAS 199, 883

[35] Blandford, R.D., McKee, C.F. \& Rees, M.J. 1977, Nature 217, 211

[36] Blandford, R. D. \& Ostriker J. P. 1978, ApJ 221, L29

[37] Blattnig, S.R. et al. 2000, Phys. Rev. D 62, 094030

[38] Blumenthal, G.R. \& Gould, R.J. 1970, Rev. Mod. Phys. 42, 237

[39] Bogovalov, S.V. et al. 2007, enviado a MNRAS [astro-ph/0710.1961]

[40] Bondi, H. \& Hoyle, F. 1944, MNRAS 104, 273

[41] Bordas, P., Paredes, J., Bosch-Ramon, V., Orellana, M., 2007, Ap\&SS 309, 339

[42] Bosch-Ramon, V., Romero, G.E. \& Paredes, J.M. 2005, A\&A 429, 267

[43] Bosch-Ramon, V., Romero, G.E. \& Paredes, J.M. 2006a, A\&A 447, 263

[44] Bosch-Ramon, V., Paredes, J.M., Romero, G.E., \& Ribó, M. 2006b, A\&A, 459, L25

[45] Bosch-Ramon, V. 2007, Ap\&SS 309, 321

[46] Butt, Y.M., Torres D. F., Combi J. A., Dame T., Romero G. E. 2001, ApJ 561, L167

[47] Butt, Y.M., Torres D.F., Romero G.E., Dame T., Combi J.A. 2002, Nature 418, 499

[48] Butt, Y.M., Maccarone, J. T. \& Prantzos, N. 2003, ApJ 587, 748

[49] Casares, J. 2005a, en Proc. "The Many Scales of the Universe - JENAM 2004 Astrophysics Reviews", Kluwer Academic Publishers

[50] Casares, J. 2005b, MNRAS 360, 1105 
[51] Coe, M.J., et al. 2006, MNRAS 368, 447

[52] Combi J.A., Romero G.E., Benaglia P., Jonas J. 2001, A\&A 366, 1047

[53] Conti, P.S. \& Ebbets, D. 1977, ApJ 213, 438

[54] Coppi, P.S.\& Blandford, R.D. 1990, MNRAS 245, 453

[55] Corbel, S., et al. 2003, A\&A 400, 1007

[56] Crowther P.A. 2007, ARA\&A 45, 171

[57] Cui, W. 2006, Proc. "Vulcano workshop: Frontier Objects in Astrophysics and Particle Physics", Editrice Compositori, Bologna, Italy

[58] Cheng, K.S., et al. 1991, ApJ 379, 290

[59] Cheng, K.S. \& Ruderman, M. 1989, ApJ 337, L77

[60] Cheng, K.S. \& Ruderman, M. 1991, ApJ 373, 187

[61] Cheng, K.S., \& Romero, G.E. 2004, Cosmic Gamma Ray Sources, Kluwer Academic Publishers, The Netherlands

[62] Christiansen, H.R., Orellana, M., \& Romero, G.E. 2006, Phys. Rev. D. 73, 063012

[63] Chernyakova, M., Neronov, A., \& Walter, R. 2006, MNRAS 372, 1585

[64] Dean, A.J. et al. 2005, A\&A 443, 485

[65] Dermer, C.D. 1986, A\&A 157, 223

[66] Dermer, C.D., Böttcher, M. 2006, ApJ 643, 108

[67] Dhawan, V., Mioduszewski, A., \& Rupen, M., 2006, en Proc. of the VI Microquasar Workshop, Como-2006

[68] Distefano, C., et al. 2002, ApJ 575, 378

[69] Domingo Santamaría, E. 2006, Ph.D. Thesis, http://www .tdx . cbuc.es/TESIS_UAB/AVALLABLE/TDX-1013106-131110/

[70] Donati, J.F., et al. 2002, MNRAS 333, 55

[71] Dougherty, S.M. \& Pittard, J.M. 2006, Proc. 8th EVN Symposium, Torun

[72] Drury, L.O'C. 1983, Rep. Prog. Phys 46, 973

[73] Dubus, G. 2006a, A\&A 456, 801

[74] Dubus, G. 2006b Proc. "Vulcano workshop: Frontier Objects in Astrophysics and Particle Physics", Editrice Compositori, Bologna, Italy

[75] Dubus, G., 2006c, A\&A 451, 9

[76] Eichler D. \& Usov, V. 1993, ApJ 402, 271

[77] Esin, A.A., McClintock, J.E. \& Narayan, R. 1997, ApJ 489, 865 
[78] Esposito, P. et al, 2007, A\&A 474, 575

[79] Falcke, H. \& Biermann, P.L. 1995, A\&A 293, 665

[80] Fender, R.P. 2006, in Compact Stellar X-Ray Sources, eds. W.H.G. Lewin and M. van der Klis, Cambridge University Press

[82] Fender, R.P., Belloni, T., Gallo, E. 2004, MNRAS 355, 1105

[82] Fender, R.P. \& Maccarone, T. 2004, en: Cosmic Gamma-ray Sources, ed. K.S: Cheng \& G.E. Romero, Kluwer Academic Publishers, Dordrecht

[83] Finger, M.H., Wilson, R.B., Harmon, A.B. 1996, ApJ 459, 288

[84] Frail, D.A. \& Hjellming, R.M. 1991, AJ 101, 2126

[85] Frank, J., King, A. \& Raine, D. 2002 Accretion Power in Astrophysics (third edition), Cambridge University Press

[86] Gaisser, T.K., Stecker, F.W., Harding A.K. \& Barnard, J.J. 1986, ApJ 309, 674

[87] Gaisser, T.K. 1990, Cosmic Rays and Particle Physics, Cambridge University Press, Cambridge

[88] Gaisser, T.K., Halzen, F., \& Stanev, T. 1995, Phys. Rept. 258, 173 [Erratum-ibid., 271, 355 (1996)]

[89] Gallo, E. et al. 2005, Nature 436, 819

[90] Gallo, E. et al. 2006, MNRAS 344, 60

[91] Gallo, E. 2007, Ap\&SS, 309

[92] Gehrels, N., et al. 2000, Nature 404, 363

[93] Ginzburg V. L. \& Syrovatskii S. I. 1964, The Origin of Cosmic Rays, Pergamon Press, Oxford

[94] Ginzburg, V.L. \& Syrovatskii, S.I. 1964, Sov. Astron. 8, 342

[95] Giovannelli, F. \& Graziati, L.S. 1992, Sp. Sci. Rev. 59, 1

[96] Goldreich, P. \& Julian W.H. 1969, ApJ 157, 869

[97] Gosh, P. \& Lamb, F.K. 1979, ApJ 234, 296

[98] Gould, R.J. \& Schréder, G.D. 1967, Pys. Rev. 155, 1404

[99] Gregory, P.C. \& Neish, C. 2002, ApJ 580, 1133

[100] Gregory, P.C. \& Taylor, A.R. 1978, Nature 272, 704

[101] Gregory, P.C. et al. 1989, ApJ 339, 1054

[102] Grenier, I.A. 2000, A\&A 364, L93

[103] Greiner, J. \& Rau, A. 2001, A\&A 375, 145 
[104] Grundstrom, E.D., et al. 2007a, ApJ 656, 437

[105] Grundstrom, E.D., et al. 2007b, ApJ 660, 1398

[106] Haigh, N.J., Coe, M.J. \& Fabregat, J. 2004, MNRAS 350, 1457

[107] Hall, T.A. et al. 2003, ApJ 583, 853

[108] Hardee, P.E. \& Hughes, P.A. 2003, ApJ 583, 116

[109] Hardrop, J. et al. 1959, Luminous Stars of the Northern Milky Way I, HamburgBengdorf

[110] Hartman, R.C., et al. 1999, ApJS 123, 79

[111] Hayasaki, K. \& Okazaki, A.T. 2004, MNRAS 350, 971

[112] Hayasaki, K. \& Okazaki, A.T. 2006, MNRAS 372, 1140

[113] Heinz, S. \& Sunyaev R. 2002, A\&A, 390, 751

[114] Hermsen, W. et al. 1977, Nature 269, 494

[115] Hill, A.B., et al. 2007, MNRAS [astro-ph/0708.0791]

[116] Hjellming, R.M., et al. 1999, American Astronomical Soc. Meeting 193, 103

[117] Huijerat, A., et al. 2003, A\&A 408, 415

[118] Hutching, J.B. \& Crampton, D. 1981, PASP 93, 486

[119] Ichimaru, J. 1977, ApJ 214, 840

[120] James, F. 1980, Rep. Prog. Phys 43, 73

[121] Jauch, J.M. \& Rohrlich, F. 1976, The Theory of Photons and Electrons, SpringerVerlag, Berlin

[122] Jones, F. 1968, Physical Review 167, 1159

[123] Jokipii, J.R. 1987, ApJ 313, 842

[124] Joss, P.C. \& Rappaport S. A. 1984, ARA\&A 22, 537

[125] Kaiser, C.R., Sunyaev, R., Spruit, H.C. 2000, A\&A 356, 975

[126] Kamae, T., Abe, T. \& Koi, T. 2005, ApJ 620, 244

[127] Karle, A. et al. (ICECUBE coll.) 2002, Proc. "XXth International Conference on Neutrino Physics and Astrophysics", Munich

[128] Katz, J.I. 1980, ApJ 236, L127

[129] Kaufman Bernadó, M.M., Romero, G.E., \& Mirabel, I.F. 2002, A\&A 385, L10

[130] Kaufman Bernadó, M.M., 2004 PhD. Thesis, Universidad de Buenos Aires [astro$\mathrm{ph} / 0504498]$

[131] Kelner, S.R., Aharonian, F. A., Bugayov V.V. 2006, Phys.Rev. D 74, 034018 
[132] Khangulyan, D., Aharonian, F. \& Bosch-Ramon, V. 2007, enviado a MNRAS [astro$\mathrm{ph} / 0707.1689]$

[133] King, A.R., Pringle, J.E. \& Livio, M. 2007, MNRAS en prensa [astro-ph/0701803]

[134] Kniffen, D.A. et al. 1997, ApJ 486, 126

[135] Kotani, T. et al. 1994, PASJ 48, 619

[136] Lamers, H.J.G.L.M. \& Cassinelli, J.P., 1999, Introduction to Stellar Winds, Cambridge

[137] Lamers, H.J.G.L.M. \& Leitherer 1993, ApJ 412, 771

[138] Landau, L.D. \& Rumer, G. 1938, Proc. R. Soc. A166, 213

[139] Larwood, J. 1998, MNRAS 299, L32

[140] Leahy, D.A., Harrison, F.A. \& Yoshida, A. 1997, ApJ 475, 823

[141] Lee, U., Saio, H. \& Osaki, Y. 1991 MNRAS 250, 432

[142] Levinson, A. \& Waxman, E. 2001, Physical Review Letters 87, Issue 17, id. 171101

[143] Li, X.D. 1997, ApJ 476, 278

[144] Lightman, A.P. \& Eardley, D.M. 1974, ApJ 187, 1

[145] Lipari, P. 1993, Astropart. Phys. 1, 195

[146] Lipunov, V.M. 1992 Astrophysics of Neutron Stars, Springer-Verlag

[147] Longair, M.S., High Energy Astrophysics, Vol I (1992) y II (1994), Cambridge Univesity Press, Cambridge

[148] Maccarone, J.T. 2002, MNRAS 336, 1371

[149] Maccarone, J.T. 2005, MNRAS 364, 971

[150] Mannheim, K. \& Schlickeiser, R. 1992, A\&A 286, 983

[151] Maier, G. et al. (VERITAS coll.) 2007, Proc. ICRC, en prensa [astro-ph/0709.3661]

[152] Maraschi, L. \& Treves, A., 1981, MNRAS 194, 1

[153] Margon, B. 1984, ARA\&A 22, 507

[154] Markoff, S., Nowak, M., Corbel, S., Fender, R., \& Falcke, H. 2003, A\&A 397, 645

[155] Markoff, S., Nowak, M. A., Wilms, J. 2005, ApJ 635, 1203

[156] Martí, J. 1993, PhD Thesis, Universitat de Barcelona

[157] Martí, J. \& Paredes, J.M. 1995, A\&A 298, 151

[158] Massi, M. et al. 2001, A\&A 376, 217

[159] Massi, M. et al. 2004, A\&A 414, L1 
[160] Massi, M. \& Kaufman-Bernado, M. 2007, A\&A, en prensa [astro-ph/0709.4287]

[161] Meier, D.L., Koide, S. \& Uchida, Y. 2001, Science 291, 84

[162] Merloni, A. \& Nayakshin, S. 2006, MNRAS 372, 728

[163] Michel, F.C. 2004, Advances in Space Research 33, 542

[164] Miller-Jones, J., Fender, R.P. \& Nakar, E. 2006, MNRAS 367, 1432

[165] Mioduszewski, A.J., et al. 2001, ApJ 553, 766

[166] Mirabel, I.F. et al. 1992, Nature 358, 215

[167] Mirabel, I.F. \& Rodríguez, L.F. 1994, Nature 392, 673

[168] Mirabel, I.F. et al. 1998, A\&A 330, L9

[169] Mirabel, I.F. \& Rodríguez, L.F. 1999, ARA\&A 37, 409

[170] Mirabel, I.F. 2006, Ap\&SS 309, 267

[171] Moskalenko, I.V. 1995, Space Science reviews 72, 593

[172] Mukadam, A.S. et al. 2007, ApJ, en prensa [astro-ph/0707.2094]

[173] Narayan, R. \& Yi, L. 1994, ApJ 428, L13

[174] Narayan, R. \& Yi, L. 1995 ApJ 444, 231

[175] Narayan, R., Mahadevan, R., \& Quataert, E. 1998, Theory of Black Hole Accretion Disks, Cambridge University Press, Cambridge

[176] Negueruela I. 1998, A\&A 338, 505

[177] Negueruela, I. \& Okazaki, A.T. 2001, A\&A 369, 108

[178] Neronov, A., \& Chernyakova, M. 2007, enviado al MNRAS [astro-ph/0701144]

[179] Ogilvie, G.I., \& Dubus, G. 2001, MNRAS 320, 485

[180] Okazaki, A.T. 1991, PASJ 43, 75

[181] Okazaki, A.T., Bate, M.R., Ogilvie, G.I \& Pringle J.E. 2002, MNRAS 337, 967

[182] Orellana, M. \& Romero, G.E. 2005, Ap\&SS 297, 167

[183] Orellana, M., Romero, G.E. \& Pellizza, L.J. 2006, BAAA 49, 330

[184] Orellana, M. \& Romero, G.E. 2007, Ap\&SS 309, 333

[185] Orellana, M. et al. 2007a, A\&A 476, 9

[186] Orellana, M. Romero, G.E., Pelliza, L., Vidrih, S. 2007b, A\&A 465, 703

[187] Pacholczyk, A. G. 1970, Radio Astrophysics, Freeman, San Francisco

[188] Paczynski, B. 1971, ARA\&A 9, 183

[189] Paredes, J.M. 1987, PhD Thesis, Universitat de Barcelona 
[190] Paredes, J.M., Estalella, R. \& Rius, A. 1990, A\&A 237, 377

[191] Paredes, J.M. et al. 1994, A\&A 288, 519

[192] Paredes, J.M., Martí, J., Peracaula, M. \& Ribó, M. 1997, A\&A 320, L25

[193] Paredes, J. M., Martí, J., Ribó, M., \& Massi, M. 2000, Science 288, 2340

[194] Paredes, J.M. 2005, Chinese Journal of Astronomy \& Astrophysics, Vol. 5 Supplement, 121

[195] Paredes, J.M., Bosch-Ramon, V. \& Romero G.E., 2006, A\&A 451, 259

[196] Pecaraula, M. et al. 1997, A\&A 328, 283

[197] Perucho, M. \& Bosch-Ramon, V. 2007, en preparación

[198] Pfrommer, C. \& Enslin, T.A. 2004, A\&A 413, 17

[199] Pooley, G. G., Fender, R. P., Brocksopp, C. 1999, MNRAS 302, L1

[200] Porter, J.M. 1999, A\&A 348, 512

[201] Porter, J.M. \& Rivinius, T. 2003, PASP 115, 1153

[202] Poutanen, J. 1998, Theory of Black Hole Accretion Discs, eds. M.A. Abramowicz, G.

Bjornsson, J.E. Pringle [astro-ph/9805025]

[203] Protheroe, R.J. 1986, MNRAS 221, 769

[204] Protheroe, R.J. 1998, ADP-AT-98-9 [astro-ph/9812055]

[205] Protheroe, R.J., Mastichiadis, A. \& Dermer, C.D. 1992, Astroparticle Physics 1, 113

[206] Purmohammad, D. \& Samimi, J. 2001, A\&A 371, 61

[207] Pringle, J.E. 1981, ARA\&A 19, 137

[208] Quirrenbach, A. et al. 1993, ApJ 416, L25

[209] Rees, M.J. 1978, MNRAS 184, 61

[210] Reimer, O. \& Funk, S. 2007, Ap\&SS 309, 203

[211] Remillard, R.E. \& McClintock, J.E. 2006, ARA\&A 44, 49

[212] Romero, G.E., Benaglia P., Torres D.F. 1999, A\&A 348, 868

[213] Romero, G.E., et al. 2001, A\&A 376, 599

[214] Romero, G.E. \& Torres, D.F. 2003, ApJ 586, L33

[215] Romero, G.E., Torres, D.F., Kaufman-Bernadó, M.M. \& Mirabel, I.F. 2003, A\&A 410, L1

[216] Romero, G.E., et al. 2004, ESA-SP 552, 703

[217] Romero, G.E. \& Orellana, M. 2005, A\&A 439, 237 
[218] Romero, G.E., Okazaki, A.T., Orellana, M. \& Owocki S.P. 2007a, A\&A 474, 15

[219] Romero, G.E., et al. 2007b, Proc Clumping in Hot Star Winds, en prensa [astro$\mathrm{ph} / 0708.1525]$

[220] Rossi, B. \& Greisen, K., 1941, Reviews on Modern Physics 13, 240

[221] Russell, D.M., et al, 2007, MNRAS 376, 134

[222] Sarazin, C.L., Begelman, M.C., \& Hatchett, S.P. 1980, ApJ 238, L129

[223] Shakura, N.I. \& Sunyaev, R.A. 1973, A\&A 24, 337

[224] Shapiro, S.L. \& Teukolsky S. A. 1983, Black Holes, White Dwarfs and Neutron Stars,

Wiley-interscience, New York

[225] Sidoli, L., et al. 2006, A\&A 459, 901

[226] Sierpowska, A. \& Bednarek, W. 2005, MNRAS 356, 711

[227] Smith, A. et al.(VERITAS Coll.) 2007, Proc. ICRC'07 en prensa [astro-ph/0709.4299]

[228] Stephens, S.A. \& Badhwar, G.D. 1981, Ap\&SS 76, 213

[229] Szostek, A. \& Zdziarski, A.A. 2007, MNRAS 375, 793

[230] Starwarz, L. \& Ostrowski, M. 2002, ApJ 578, 763

[231] Stecker, F.W. 1970, Ap\&SS 6, 377

[232] Stecker, F.W. 1979, ApJ 228, 919

[233] Stella, L., White, N.E., Rosner, R. 1986, ApJ 208, 669

[234] Sturrock, P. 1971, ApJ 164, 529

[235] Svenson, R. 1987, MNRAS 227, 403

[236] Swanenburg, B.N., Bennett, K., Bignami, G.F., et al. 1981, ApJ 243, L69

[237] Taylor, A.R. \& Gregory, P.C. 1982, ApJ 255, 210

[238] Taylor, A.R. et al. 1992, ApJ 395, 268

[239] Taylor, A.R. et al. 1996, A\&A 305, 817

[240] Teresi, V., Molteni, D., Toscano, E. 2004, MNRAS 351, 297

[241] Torres, D.F., Domingo Santamaría, E., Romero, G.E. 2004, ApJ L75

[242] Torres, D.F., Romero, G.E., Combi, J.A., et al. 2001, A\&A 370, 468

[243] Torres, D.F., Romero, G.E., Dame, T., et al. 2003, Physics Reports 382,303

[244] Torres, D.F. \& Halzen, F. 2007, Astroparticle Physics 27, 500

[245] Tudose, V. et al. 2006, MNRAS 372, 417

[246] van der Hucht, K.A. 2001, New Atronomy Rev. 45, 135 
[247] Völk, H.J. \& Forman, M. 1982, ApJ 253, 188

[248] Volkova, L.V. 1980, Sov. J. Nucl. Phys. 31, 784

[249] Wang, Y.M. 1996, ApJ 465, L111

[250] Waters, L.B.F.M. 1986, A\&A 162, 121

[251] Waters L.B.F.M., Cote J., Larmers H.J 1987, A\&A 185, 206

[252] Weber, E.J. \& Davis, L. 1967, ApJ 148, 217

[253] White, R.L. 1985, ApJ, 289, 689

[254] Wijers, R.A.M.J. \& Pringle, J.E. 1999, MNRAS 308, 207

[255] Zamanov, R.K. 1995, MNRAS 272, 308

[256] Zhang, F., Li, X.D. \& Wang, Z.R. 2004, ApJ 603, 663

[257] Zanni, C. et al. 2007, A\&A 469, 811

[258] Zdiarski, A.A. 1988, ApJ 289, 514

[259] Zdziarski, A.A., Poutanen, J., Paciesas, W.S. \& Wen, L. 2002, ApJ 578, 357 


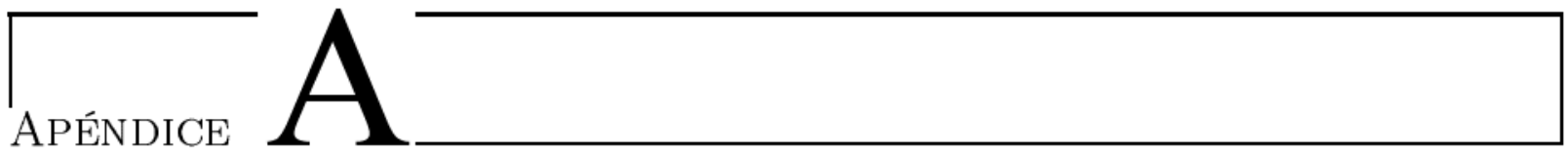

\section{Procesos físicos / Cálculos numéricos}

\section{A.1. Aceleración de partículas en frentes de choque}

En el contexto de esta tesis, los mecanismos considerados para la aceleración de partículas hasta muy altas energías son la aceleración en una diferencia de potencial electrostática y la aceleración difusiva en frentes de choque1. Las ondas de choque, como es sabido, son perturbaciones en un plasma que se propagan a una velocidad superior a la del sonido en el medio. Producen una discontinuidad en las propiedades del plasma a uno y otro lado de la superficie que llamamos frente de choque2. Las cantidades físicas del fluído a ambos lados del frente de choque se relacionan mediante las leyes de conservación de masa, momento y energía a través de la discontinuidad. En el sistema de referencia fijo al frente de choque la situación es (en el caso plano ideal) estacionaria, y si rotulamos con 1 y 2 las cantidades a ambos lados del frente de choque, puede definirse la razón de compresión como $r=\rho_{1} / \rho_{2}$. Este cociente de densidades también cumple

$$
r=\frac{v_{1}}{v_{2}}=\frac{\gamma+1}{(\gamma-1)+2 / M_{1}^{2}}
$$

donde $M=v / c$ es el número de Mach, y $\gamma$ el coeficiente adiabático. En el límite de choques fuertes $(M \gg 1)$ y gas ideal monoatómico $(\gamma=5 / 3)$, resulta $r \rightarrow 4$. Si la velocidad del frente de choque en el sistema de laboratorio es $v_{s}$, la velocidad $v_{p}$ del fluído que la onda pone en movimiento (post-choque) cumple $v_{s} / v_{p} \simeq r /(r-1)$.

A fines de los años 1970, modificando una idea originalmente de Fermi, varios autores desarrollaron independientemente la idea de un mecanismo de aceleración de partículas en

\footnotetext{
${ }^{1}$ No hemos considerado la aceleración que puede tener lugar a través de otros procesos (ver ej. Jokipii 1987) como ser reconexión magnética, que se supone son menos eficientes que la aceleración difusiva en los escenarios considerados.

${ }^{2}$ Cuyo espesor es en rigor de algunos: a) caminos libres medios colisionales en gases comunes, b) giroradios de un ion en plasmas tenues, o c) longitudes de Debye si hay efectos electrostáticos importantes. Para una descripción más detallada de la teoría de aceleración difusiva ver Drury (1983).
} 
ondas de choque en ambientes astrofísicos lo bastante eficiente como para dar cuenta de la aceleración de los rayos cósmicos. Una partícula de alta energía difunde en cualquiera de los lados del frente por dispersión en las irregularidades y turbulencias magnéticas. En el sistema de referencia fijo al fluído (a ambos lados del frente) la distribución de impulso lineal es isotropizada por las dispersiones. Usando transformaciones relativistas simples, Protheroe (1998) brinda una clara deducción de la ganancia neta de energía que experimenta una partícula al completar un ciclo de cruce (ver Figura A.1), yendo de la región post-choque a la pre-choque y volviendo a la post-choque,

$$
\frac{\langle\Delta E\rangle}{E} \simeq \frac{4}{3} \frac{(r-1)}{r} \frac{v_{s}}{c} .
$$

Esta ganancia es de primer orden en $\beta_{s}=v_{s} / c$ por lo que este mecanismo se conoce también como mecanismo de Fermi de primer orden 3 .

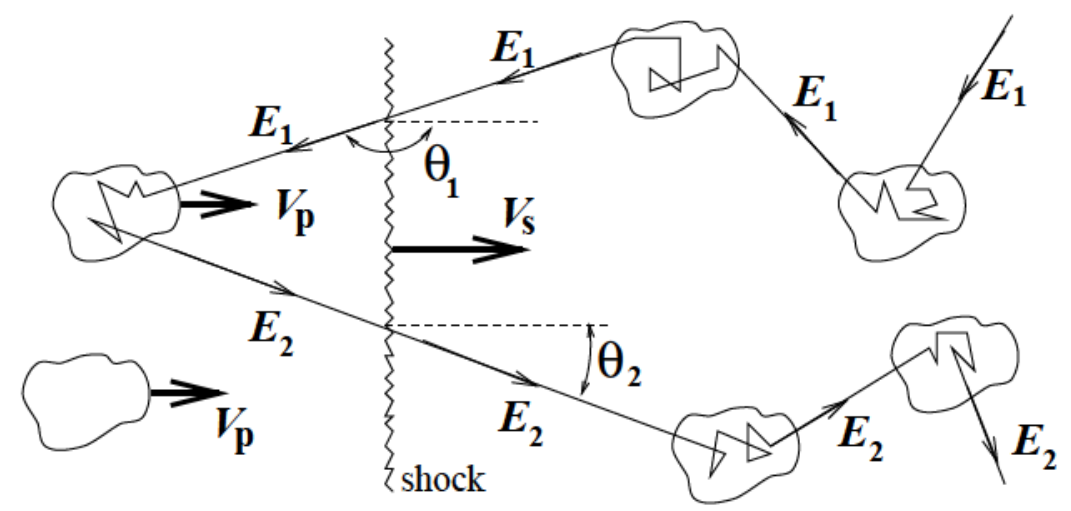

Figura A.1: Esquema de la trayectoria de una partícula que atraviesa un frente de choque, con energía inicial $E_{1}$. Luego de ser dispersada vuelve a la región original con energía $E_{2}$ (Protheroe 1998).

Mientras que una partícula inicialmente delante de la onda de choque siempre lo atravesará, una vez del otro lado existe cierta probabilidad $\left(P_{\text {esc }} \approx 4 v_{s} / r\right)$ que sea advectada y nunca vuelva a cruzarlo. Si la energía inicial de la partícula es $E_{0}$, después de $k$ cruces resulta:

$$
E=E_{0}\left(1+\frac{\langle\Delta E\rangle}{E}\right)^{k}
$$

El número de partículas en la región de aceleración con energía mayor que $E$ es

$$
\frac{d n}{d E}(>E) \propto\left(1-P_{\mathrm{esc}}\right)^{k}
$$

\footnotetext{
${ }^{3}$ Para deducir A.2 debe asumirse que la partícula ya es relativista, y por eso suele hablarse de reaceleración.
} 
donde, por la expresión anterior, $k=\frac{\ln \left(E / E_{0}\right)}{\ln (1+\langle\Delta E\rangle / E)}$. Tomando logaritmo, puede escrirse

$$
\ln \left[\frac{d n}{d E}(>E)\right]=B-(\Gamma-1) \ln E,
$$

donde $B$ es una constante y

$$
\Gamma=1-\frac{\ln \left(1-P_{\mathrm{esc}}\right)}{\ln (1+\langle\Delta E\rangle / E)} \sim \frac{r+2}{r-1} .
$$

Entonces, en su forma diferencial, la distribución espectral de las partículas aceleradas en el frente de choque sigue una ley de potencias,

$$
\frac{d n}{d E}(E) \propto E^{-\Gamma}
$$

En el caso de un frente de choque fuerte y gas monoatómico se obtiene $\Gamma=2$. En choques donde el gas es relativista $(\gamma=4 / 3)$ se obtiene $\Gamma=1.5$, aunque un planteo más realista arroja valores algo mayores, entre 1.5 y 2 (Protheroe 1998). La aceleración difusiva de partículas es un tema mucho más amplio de lo aquí expuesto, para tratamientos de efectos no-lineales (mediante simulaciones numéricas) ver ej. Baring et al. (2005) y sus referencias.

En cuanto a la tasa de ganancia de energía que experimenta una partícula mediante este mecanismo, debe calcularse

$$
\left.\frac{d E}{d t}\right|_{\text {acc }}=E \frac{\langle\Delta E\rangle}{E} \frac{1}{t_{\text {ciclo }}}
$$

donde $t_{\text {ciclo }}$ es lo que tarda la partícula en completar un ciclo de cruce. Este valor se estima haciendo hipótesis sobre el coeficiente de difusión. El resultado depende en parte de la orientación del campo magnético respecto a la normal del frente de choque, obteniéndose una expresión como (9.5). En general, para partículas con carga $Z e$ se obtiene (en unidades cgs)

$$
\left.\frac{d E}{d t}\right|_{\mathrm{acc}}=\xi Z e B \quad \text { con } \xi<1 \text {. }
$$

\section{A.2. Interacciones hadrónicas}

Nos hemos focalizado en la emisión gamma originada por la interacción de protones relativistas con el medio local, le reservamos este espacio a detalles técnicos relacionados con este proceso, si bien la teoría (QCD) que describe en detalle la interacción $p p$ está fuera de alcance de los estudios de esta tesista.

Los principales canales de la interacción protón-protón dan como resultado la creación de mesones $\pi$. Los piones tienen una vida media corta $\left(2.6 \times 10^{-8}\right.$ segundos para un $\pi^{ \pm}$ y $8.4 \times 10^{-17}$ s para un $\pi^{0}$ ) transcurrida la cual decaen produciendo muones, electrones, 
positrones y fotones como se ha detallado en el texto, siendo la vida media del muón $2.2 \times$ $10^{-6} \mathrm{~s}$. La energía umbral para que un protón interactúe en forma inelástica, produciendo un $\pi^{0}$ es

$$
E_{\mathrm{th}}=\left(m_{p}+2 m_{\pi}+\frac{m_{\pi}^{2}}{2 m_{p}}\right) c^{2}=1.22 \mathrm{GeV}
$$

Dada una expresión arbitraria para el flujo de protones $J_{p}(E)$ que interatúan con protones o núcleos atómicos en reposo, la emisividad $\gamma$ (el número de fotones por unidad de energía, volumen y tiempo) que resulta del decaimiento de $\pi^{0}$ involucra, a través de la expresión (5.10) para la tasa de creación de piones, a la sección eficaz diferencial inclusiva de la interacción $p p$. Varios autores han tratado con este problema, desde Ginzburg \& Syrovatskii (1964) que recalcaron la importancia del proceso en el ámbito de la emisión $\gamma$ difusa que emiten las galaxias, por interacción de rayos cósmicos con el material del medio interestelar.

Stecker (1970) señala que existen dos modos para la creación de los piones. Una componente involucra una isobara $\Lambda^{+}$como partícula intermediariat, que decae según $\Lambda^{+} \rightarrow \pi^{0}+p$, donde el protón se lleva aproximadamente la mitad de la energía del protón relativista inicial. El resto de la energía disponible en el sistema centro de momentos (CM) de la colisión forma un "gas" de piones con distribución tipo Maxwell-Boltzmann, a la que suele llamarse fireball haciendo referencia a la idea original de Fermi. En el CM, el fireball está compuesto en partes iguales por mesones $\pi^{+}, \pi^{-}$y neutros.5. A éstos nos hemos referido en el Capítulo 8 y tienen una multiplicidad $\xi_{\pi} \propto E_{p}^{1 / 4}$. Cuando el protón relativista tiene una energía relativamente baja (algunos $\mathrm{GeV}$ ) es difícil distinguir las dos componentes, mientras que a energías mayores el canal de la isobara se lleva más energía, dando lugar a un pión neutro "lider" en el sentido de más energético que los demás.

Trabajos como por ej. Dermer (1986), Stephen \& Badhwar (1981), Blattnig et al. (2000), Kamae et al. (2005) brindan distintas parametrizaciones para la sección eficaz $p p$, ajustando datos experimentales de aceleradores de partículas. Más allá de las diferencias entre estas parametrizaciones (ver el apéndice que brinda Domingo Santamaría 2006), la parametrización más simple y que brinda ventajas de cálculo por ser analítica, además de proveer bajos errores a energías $\lesssim 100 \mathrm{GeV}$, parece seguir siendo la aproximación de la función $\delta$ de Aharonian \& Atoyan (2000). Este formalismo supone que una fracción promedio $\kappa$ de la energía cinética del protón incidente se transfiere a un pion neutro, que es el que genera el término principal en la emisividad $\gamma$. Específicamente, se tiene

$$
\frac{d \sigma}{d E_{\pi^{0}}}\left(E_{\pi^{0}}, E_{p}\right)=\sigma_{\text {inel }}\left(E_{p}\right) \delta\left(E_{\pi^{0}}-\kappa\left(E_{p}-m_{p} c^{2}\right)\right) .
$$

En esta expresión, $\sigma_{\text {inel }}$ es la sección eficaz inelástica, cuya parametrización más reciente hemos dado en (8.5), y se grafica en la Figura A.2 En un rango muy amplio de energías

\footnotetext{
${ }^{4} \mathrm{La}$ isobara suele llamarse estado de resonancia. Es una partícula con el mismo contenido de quarks que en este caso, el protón. Tiene un decaimiento fuerte en $\tau_{\Lambda} \approx 10^{-23} \mathrm{~s}$.

${ }^{5}$ En rigor, quizás sea más correcto hablar del fireball en términos de un plasma de quarks y gluones, como lo mencionan Pfrommer \& Enslin (2004).
} 
$\kappa \approx 0.17$. La aproximación delta brinda mejores resultados cuando el flujo incidente de protones $J_{p}$ sigue una ley suave de la energía (Kelner et al. 2006).

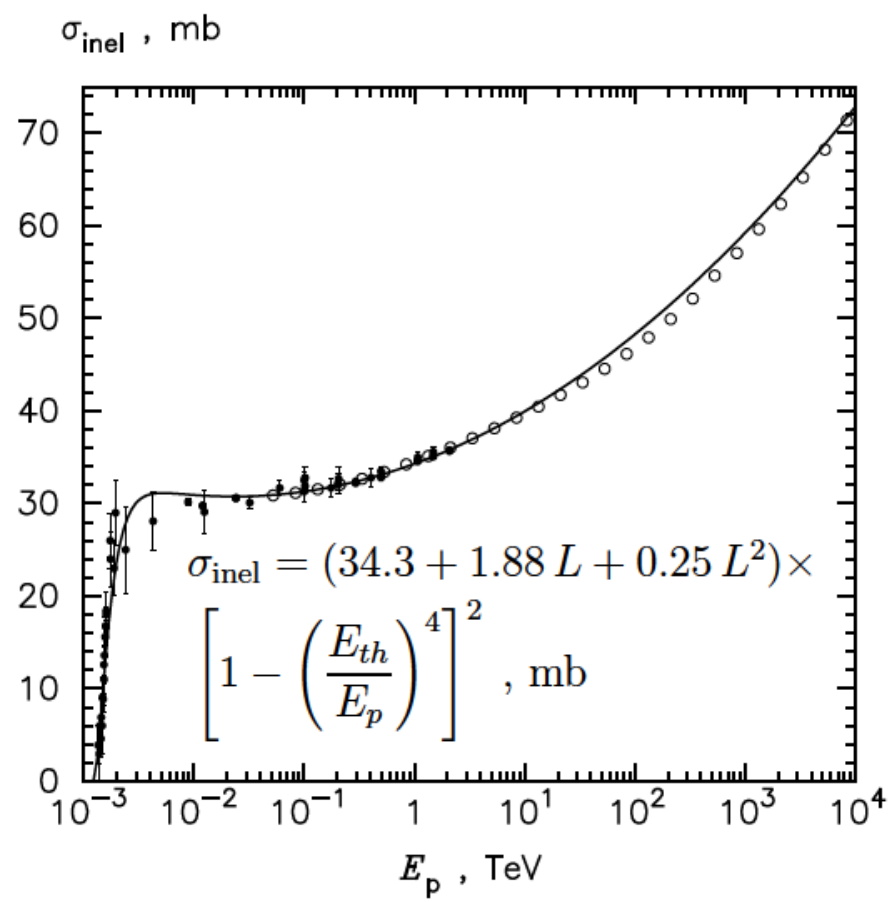

Figura A.2: Sección eficaz de la interacción $p-p$, Kelner et al. (2006). Se incluyen los datos experimentales con su barra de error, y en cículos blancos la sección eficaz estimada con un código de simulación de interacciones (SIBYLL).

Ya desde un análisis cinemático los decaimiento $\pi^{ \pm} \rightarrow \mu^{ \pm} \rightarrow e^{ \pm}$se presentan más complejos, y nos los reproduciremos aquí. Fueron llevados a cabo por Ginzburg \& Syrovatskii (1964), pero también existen tratamientos más actuales como el que citamos en la Sección 8.1 por Kelner et al. (2006). En la Figura A.3 se muestran las distribuciones en energía que siguen los diferentes productos del decaimiento de piones, normalizadas a $\int_{0}^{1} d w=1$, en el sistema de laboratorio.

\section{A.3. Cálculo detallado de la absorción en el campo ra- diativo estelar}

Hemos aplicado en nuestras estimaciones más recientes y en el código de simulación de cascadas, las siguientes expresiones dadas por Dubus (2006) para el cálulo de la opacidad a la propagación de un fotón $\gamma$.

La probabilidad diferencial de absorción que experimenta un rayo $\gamma$ con energía $E$, ubicado en la posición P y que se mueve en la dirección $\breve{e}_{\gamma}$ debido a fotones con energía $\epsilon$ 


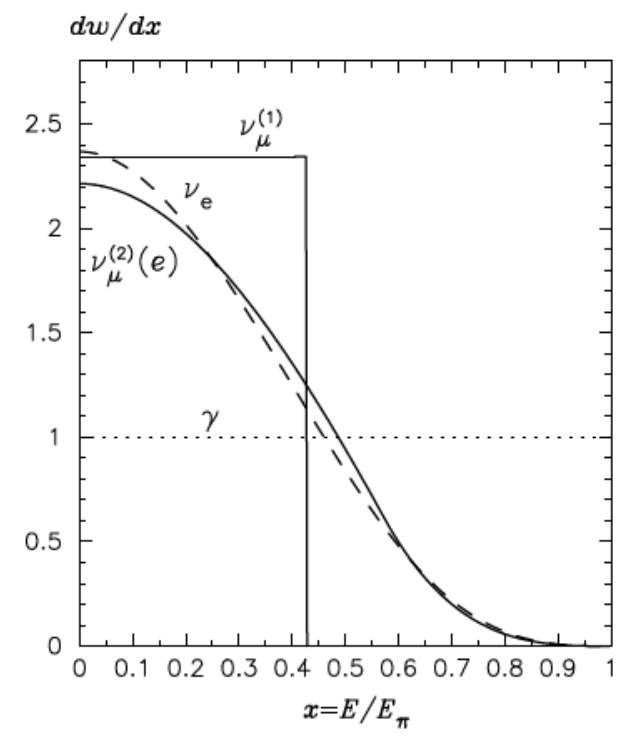

Figura A.3: Distribución de los productos secundarios en el decimiento de un pión. Kelner et al. (2006).

emitidos a lo largo de $\breve{e}_{\star}$ por un elemento de superficie de la estrella, ubicado en S es

$$
\mathrm{d} \tau_{\gamma \gamma}=\left(1-\breve{\mathbf{e}}_{\gamma} \cdot \breve{\mathbf{e}}_{\star}\right) n_{\epsilon} \sigma_{\gamma \gamma} \mathrm{d} \epsilon \mathrm{d} \Omega \mathrm{d} l
$$

donde $\mathrm{d} \Omega$ es el ángulo sólido de los fotones emitidos en $\mathrm{S}, \mathrm{y} n_{\epsilon}$ es la densidad de la radiación (estelar) en fotones $\mathrm{cm}^{-3} \mathrm{erg}^{-1} \mathrm{sr}^{-1}$.

La sección eficaz de la interacción de dos fotones, $\sigma_{\gamma \gamma}$, depende sólo de la energía total en el sistema CM, que adimensionalizada es

$$
s=\frac{\epsilon E}{2 m_{e}^{2} c^{4}}\left(1-\breve{\mathrm{e}}_{\gamma} \cdot \breve{\mathrm{e}}_{\star}\right)
$$

y la interacción ocurre sólo si $s>1$. De esta condición puede obtenerse la energía mínima, $\epsilon_{\min }$ para que los fotones estelares sean capaces de absorber al fotón con energía $E$. La sección eficaz viene dada por (Gould \& Schréder 1967)

$$
\sigma_{\gamma \gamma}=\frac{\pi r_{0}^{2}}{2}\left(1-\beta^{2}\right)\left[2 \beta\left(\beta^{2}-2\right)+\left(3-\beta^{4}\right) \ln \left(\frac{1+\beta}{1-\beta}\right)\right],
$$

con

$$
\beta=\left(1-\frac{1}{s}\right)^{1 / 2}
$$

y se ha utilizado la expresión del radio clásico del electrón $r_{0}=e^{2} / m_{e} c^{2}$. 
La emisión de la estrella puede considerarse normalmente como de cuerpo negro. Si la estrella tiene radio $R_{\star}$ y temperatura $T_{\star}$, entonces

$$
n_{\epsilon}=\frac{2 \epsilon^{2}}{h^{3} c^{3}} \frac{1}{\exp \left(\epsilon / k T_{\star}\right)-1} \mathrm{ph} \mathrm{cm}^{-3} \mathrm{erg}^{-1} \mathrm{sr}^{-1} .
$$

Orientando el sistema de referencia centrado en la estrella de modo que el fotón $\gamma$ se propague en el plano $x$ - $z$, entonces la ubicación y dirección de éste quedan determinadas por el ángulo $\psi$ y la distancia $d$. En función de los ángulos definidos en la Figura A.4 resulta

$$
1-\breve{\mathbf{e}}_{\gamma} \cdot \breve{\mathbf{e}}_{\star}=1+\cos \psi \cos \theta+\sin \psi \cos \phi \sin \theta .
$$

El ángulo sólido de los fotones que viajan en dirección $\breve{e}_{\star}$ es

$$
\mathrm{d} \Omega=\sin \theta \mathrm{d} \phi \mathrm{d} \theta
$$

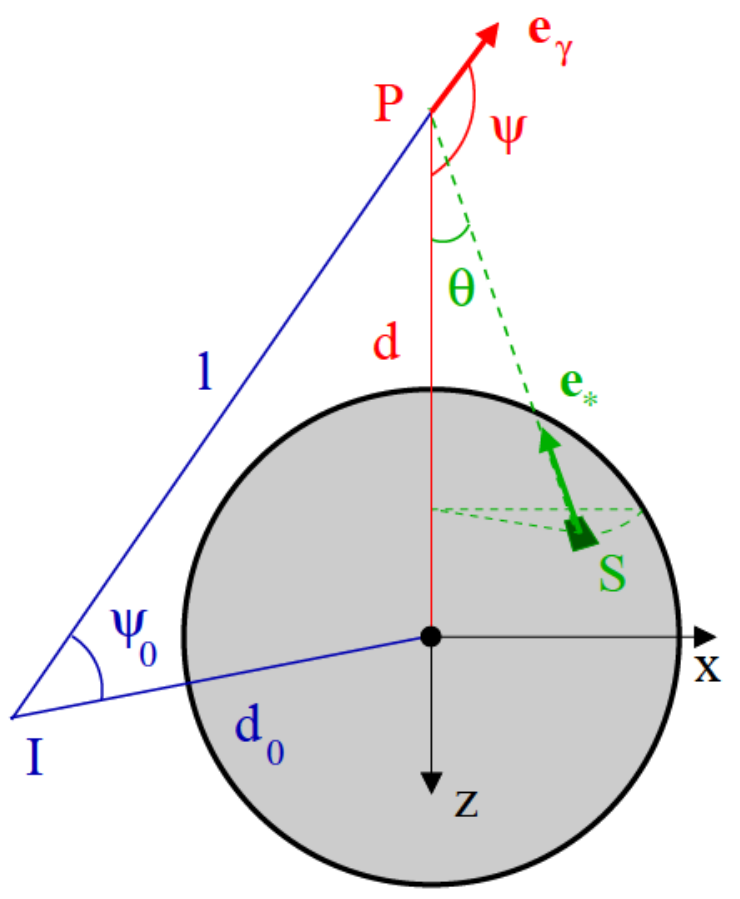

Figura A.4: Geometría adoptada para el cálculo de la opacidad del campo radiativo estelar. La absorción ocurre en $\mathrm{P}$, mediante interacción con fotones emitidos en $\mathrm{S}$. El fotón gamma es emitido en I, y recorre una distancia $l$ hasta llegar a $\mathrm{P}$. El plano $x-z$ es el definido por el centro de la estrella y el camino del rayo $\gamma$. Figura de Dubus (2006).

La distancia que el rayo gamma recorre desde el lugar donde es emitido se ha llamado $l$. Para el lugar de emisión (punto I) la distancia es $d_{0}$ y el ángulo respecto a la estrella, $\psi_{0}$, 
es nulo nulo si el fotón viaja directamente hacia la estrella. Estas cantidades se relacionan según

$$
\psi=\tan ^{-1}\left(\frac{d_{0} \sin \psi_{0}}{d_{0} \cos \psi_{0}-l}\right) \quad \text { para } l<d_{0} \cos \psi_{0},
$$

y $\psi=\pi+\tan ^{-1}(\ldots)$ para $l>d_{0} \cos \psi_{0}$. La distancia $d$ cumple $d^{2}=d_{0}^{2}+l^{2}-2 d_{0} l \cos \psi_{0}$.

La dependencia de $\mathrm{d} \tau_{\gamma \gamma} \operatorname{con} E, \epsilon, \phi, \theta$ y $l$ es ahora explicita. La opacidad para la propagación hasta un observador lejano resulta de integrar

$$
\tau_{\gamma \gamma}=\int_{0}^{\infty} \mathrm{d} l \int_{c_{\min }}^{1} \mathrm{~d} \cos \theta \int_{0}^{2 \pi} \mathrm{d} \phi \int_{\epsilon_{\min }}^{\infty} \frac{\mathrm{d} \tau_{\gamma \gamma}}{\mathrm{d} \epsilon \mathrm{d} \Omega \mathrm{d} l} \mathrm{~d} \epsilon,
$$

donde $c_{\min }=\left(1-R_{\star}^{2} / d^{2}\right)^{1 / 2}$. Para realizar este cálculo conviene cambiar de variable $\mathrm{y}$ trabajar con $\beta$ definido arriba y la expresión (A.14), donde $\beta$ varía en el intervalo $[0,1]$. Además, convendrá transformar la integral en $l$ en una integral definida sobre $\psi$ entre $\left[\psi_{0}, \pi\right]$ bajo la condición $d_{0} \sin \psi_{0} \geq R_{\star}$ (o el fotón gamma impactará la estrella).

Cuando $R_{\star} \ll d$, la integral (A.20) sobre los ángulos $\theta$ y $\phi$ devuelve la aproximación de fuente puntual

$$
\mathrm{d} \tau_{\gamma \gamma}=\pi\left(\frac{R_{\star}}{d}\right)^{2} \sigma_{\gamma \gamma} n_{\epsilon}(1+\cos \psi) \mathrm{d} l \mathrm{~d} \epsilon,
$$

donde la energía umbral es $\epsilon_{\min }=2 m_{e}^{2} c^{4} / E(1+\cos \psi)$. Esta aproximación funciona razonablemente bien a menos que $\psi \approx \pi$, o que la distancia sea $d-R_{\star}<R_{\star}$ en el punto más cercano de la trayectoria a la estrella. En estos casos, será preferible un cálculo completo de (A.20).

El cálculo de la opacidad que ofrece el campo radiativo de un disco plano es similar al caso de la estrella. La Figura A.5 muestra la geometría considerada. En este caso la trayectoria del fotón gamma inicia en el punto I, con valores iniciales $d_{0}$ y $\psi_{0}$ y mantiene al desplazarse un ángulo $\Phi$ respecto del eje $z$ de simetría del disco. La expresión (A.18) cambia a

$$
\mathrm{d} \Omega=|\cos \eta| \frac{R \mathrm{~d} R}{\tilde{l}^{2}} \mathrm{~d} \varphi .
$$

Becker \& Kafatos (1995) obtienen la relación geométrica

$$
\cos \eta=-\frac{\cos (\psi+\Phi) d}{\tilde{l}}
$$

donde

$$
\tilde{l}^{2}=d^{2}+R^{2}+2 d R \sin \psi \sin (\psi+\Phi) .
$$

Los cambios de variables son similares al caso del campo radiativo estelar, pero la integral de ángulo sólido incorpora los límites de la variable $R$ entre entre los radios interno y externo del disco. 


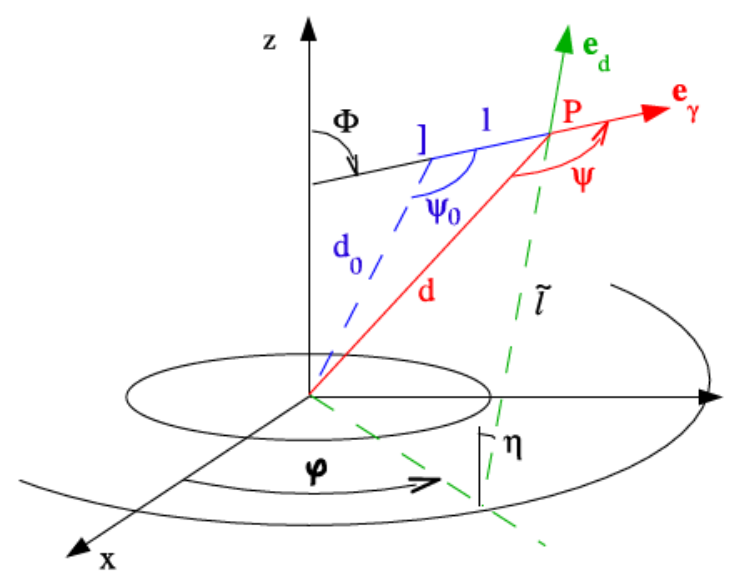

Figura A.5: Geometría adoptada para el cálculo de la opacidad del campo radiativo de un disco plano. Para la definición de los ángulos y distancias se ha seguido una convención similar a la de la Figura A.4.

\section{A.3.1. Dispersiones IC en un campo anisotrópico}

Notese que en forma similar a (A.12) la probabilidad de que un electrón interactúe con un fotón estelar por dispersión Compton inversa está dada por

$$
\mathrm{d} \tau_{e \gamma}=\left(1-\beta_{e} \breve{\mathbf{e}}_{e} \cdot \breve{\mathbf{e}}_{\star}\right) n_{\epsilon} \sigma_{e \gamma} \mathrm{d} \epsilon \mathrm{d} \Omega \mathrm{d} l,
$$

donde $\breve{\mathbf{e}}_{e}$ es ahora el versor de la dirección de movimiento del electrón que tiene velocidad $\beta_{e}$ en unidades de $c$. El parámetro de la sección eficaz IC es

$$
\eta=\frac{\epsilon E_{e}}{\left(m c^{2}\right)^{2}}\left(1-\beta_{e} \breve{\mathrm{e}}_{e} \cdot \breve{\mathrm{e}}_{\star}\right),
$$

donde $E_{e}=m c^{2} / \sqrt{1-\beta_{e}^{2}}$ es la energía del electrón. La sección eficaz fue calculada por Klein y Nishina en 1929, y vale

$$
\begin{aligned}
\sigma_{e \gamma} & =\frac{3}{4} \sigma_{T}\left\{\frac{1+\eta}{\eta^{3}}\left[\frac{2 \eta(1+\eta)}{1+2 \eta}-\ln (1+2 \eta)\right]+\frac{1}{2 \eta} \ln (1+2 \eta)-\frac{1+3 \eta}{(1+2 \eta)^{2}}\right\} \\
& \simeq \sigma_{T}(1-2 \eta) \quad \text { if } \eta \ll 1 \\
& \simeq \frac{3 \sigma_{T}}{8 \eta}\left[\frac{1}{2}+\ln (2 \eta)\right] \quad \text { if } \eta \gg 1 .
\end{aligned}
$$

Aquí se utilizó la sección eficaz de Thomson, $\sigma_{T}=8 \pi e^{4} /\left(3 m^{2} c^{4}\right) \approx 6.65 \times 10^{-25} \mathrm{~cm}^{2}$.

El espectro de fotones $\gamma$ que resulta de las dispersiones IC es de interés crucial en los cálculos de cascadas electromagnéticas que hemos implementado. Hemos seguido la aproximación dada por Jones (1968), modificada en forma similar a Bednarek (1997) ya que los fotones llegan al electrón en forma anisotrópica, provenientes de la superficie de la 
estrella. Para tratar este problema llamamos $\mu$ a la función menos coseno del ángulo entre las direcciones de movimiento del electrón y de un fotón con el que interactúa. Esto es

$$
\mu=\cos \psi \cos \theta+\sin \psi \cos \phi \sin \theta,
$$

donde por claridad hemos eliminado subíndices, y debe entenderse que los ángulos se refieren al movimiento del electrón. Estando $\psi$ determinado por el lugar donde ocurre la interacción, los otros ángulos varían en $0 \leq \theta \leq \theta_{\max }$ y $0 \leq \phi \leq 2 \pi$, donde $\cos \theta_{\max }=c_{\min }$ ya se ha definido. Los valores extremos que toma la función $\mu$ están entre las opciones $\left\{\cos \psi, \cos \left(\psi+\theta_{\max }\right), \cos \left(\psi-\theta_{\max }\right), 1\right\}$, siendo 1 sólo posible cuando $\psi<\theta_{\max }$. En nuestro código, mediante inspección de los valores mencionados se obtienen $\mu_{\min }$ y $\mu_{\max }$, que utilizamos como parámetros de

$$
\zeta_{1,2}\left(E_{\gamma}\right)=\frac{E_{\gamma} m c^{2}}{\epsilon \gamma\left(1+\beta \mu_{\max , \min }\right)\left(E-E_{\gamma}\right)}
$$

en una instancia donde la energía $\epsilon$ del fotón que interactúa ya es conocida6. Aquí $E_{\gamma}$ es la energía del fotón dispersado que es aún variable, y $\gamma$ es el factor de Lorentz del electrón.

El paso siguiente es hallar qué parte del intervalo entre $\zeta_{1}$ y $\zeta_{2}$ está dentro del (1 $\beta, 1+\beta)$, que es permitido por la cinemática de la interacción IC. Llamamos $\zeta_{\min }$ y $\zeta_{\max }$ a los valores extremos de tal intersección (si es no vacía). Utilizando la expresión 2-6 en Jones (1968), se puede escribir al espectro de fotones $\gamma$ que resultan de la dispersión de la siguiente forma

$$
\left.\frac{d N}{d t d E_{\gamma}} \propto \frac{E_{\gamma}}{\left(1-E_{\gamma} / E\right)}\left(\zeta-2 \ln \zeta-\frac{2}{\zeta}-\frac{\left(E_{\gamma} / E\right)^{2}}{\left(1-E_{\gamma} / E\right) \zeta}\right)\right|_{\zeta_{\min }} ^{\zeta_{\max }}
$$

para $\gamma \gg 1$. El cociente entre las energías del fotón $\gamma$ y del electrón que lo dispersó está acotado por

$$
\frac{E_{\gamma}}{E} \leq \frac{4 \epsilon \gamma}{m c^{2}+4 \epsilon \gamma}
$$

\section{A.4. Nociones sobre simulaciones Monte Carlo}

Como concepto básico, una técnica Monte Carlo es cualquiera que haga uso de una secuencia pseudo-aleatoria de números para resolver un problema. Este ha sido extensamente reconocido como un método poderoso para realizar ciertos cálculos, en especial aquellos demasiado complejos como para intentar una aproximación más convencional. Pero el que se aplique o no un método Monte Carlo en un dado problema no depende de la naturaleza estocástica del problema en sí, sino más bien del hecho de reformularlo de modo que una secuencia de números al azar intervenga en la resolución. Puede aplicarse siempre sea

\footnotetext{
${ }^{6}$ Muestreada de su correspondiente distribución.
} 
posible poner en correspondencia el resultado deseado con el comportamiento esperado de un sistema estocástico.

Un problema común suele ser el muestrear un valor $x$ de una dada distribución $p(x)$, dentro de un rango $\left(x_{1}, x_{2}\right)$. Utilizando $R$ para representar un número aleatorio cuya probabilidad se distribuye en forma uniforme en el rango $(0,1)$. Si en el rango de interés, los valores de $p$ son acotados, siempre puede utilizarse un método de rechazo para muestrear $x$ (ver ej. James 1980). El procedimiento consiste en muestrear primero de la distribución uniforme en $\left(x_{1}, x_{2}\right)$ a través del valor aleatorio $R_{1}$ y usando el cambio de variable

$$
x=x_{1}+\left(x_{2}-x_{1}\right) R_{1}
$$

y luego aceptar o rechazar $x$ en base a otro número aleatorio $R_{2}$. Se rechaza $x$ y se empieza de nuevo si

$$
R_{2}>\frac{p(x)}{p_{\max }}
$$

donde $p_{\max }$ es el valor máximo que toma $p$ en $\left(x_{1}, x_{2}\right)$.

Aunque este método puede ser usado para cualquier distribución, resulta muy ineficiente si $p(x)$ se aparta mucho de una distribución uniforme, en cuyo caso, se generan muchos valores aleatorios hasta conseguir un valor de $x$ aceptado. En la actualidad hay muchas recetas disponibles para muestrear de las distribuciones más conocidas, utilizando sólo un número pequeño de valores aleatorios. Además, para distribuciones relativamente simples, constantemente surgen nuevas reglas, y a veces, reglas más eficientes surgen de combinar otras.

Un ejemplo útil es el método de la función inversa. Dada una distribución $p(x)$, llamemos $\mathcal{P}(x)$ a su función cumulativa de probabilidad (la integral de $p$ hasta $x$ ). La función $\mathcal{P}(x)$ es no decreciente en la variable $x$, y por lo tanto tiene una función inversa. La transformación $\xi=\mathcal{P}(x)$ define una nueva variable que toma valores en el intervalo $(0,1)$. Debido a la correspondencia entre $x$ y $\xi$ sus distribuciones estan relacionadas por $p_{\xi}(\xi) d \xi=p(x) d x$. Luego,

$$
p_{\xi}(\xi)=p(x)\left(\frac{d \xi}{d x}\right)^{-1}=p(x)\left(\frac{d \mathcal{P}(x)}{d x}\right)^{-1}=1,
$$

de donde se vé que $\xi$ tiene una distribución uniforme en $(0,1)$, y puede muestrearse directamente como $\xi=R$. La variable $x$ puede luego obtenerse como $x=\mathcal{P}^{-1}(\xi)$, y toma valores en $\left(x_{1}, x_{2}\right)$ con distribución $p(x)$. Esto provee un método práctico para generar valores de $x$ usando el generador de $R$. Notese que $x$ es la (única) raiz de la ecuación

$$
\xi=\int_{x_{1}}^{x} p\left(x^{\prime}\right) d x^{\prime}
$$

que puede llamarse la ecuación de muestreo de la variable $x$. La Figura A.6 ilustra el concepto. Este procedimiento es particularmente útil para distribuciones $p(x)$ que son funciones analíticas simples y permiten resolver también analíticamente la ecuación (A.35).

\footnotetext{
${ }^{7}$ En el lenguaje de programación FORTRAN que se utilizo, la secuencia rand(0) tiene esa propiedad.
} 


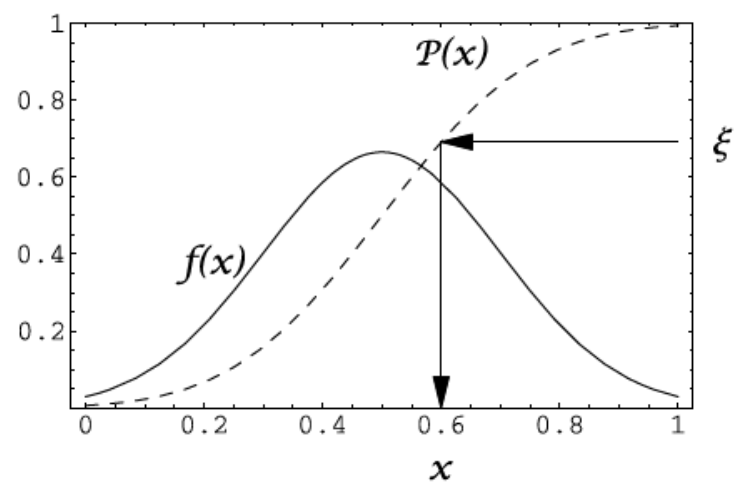

Figura A.6: Método de la función inversa para el muestreo de una variable aleatoria $x$ con ditribución de probabilidad $f(x)$.

A veces la distribución muestrear puede escribirse como suma de otras dos distribuciones para las que se conocen reglas de muestreo, por ej. $q(x) \propto\left[q_{1}(x)+q_{2}(x)\right]$ donde ambos términos varían en $(0,1)$. Entonces se muestrea ya sea de $q_{1}$ o de $q_{2}$ con un cociente de veces

$$
\alpha=\frac{\int_{0}^{1} q_{1}(x) d x}{\int_{0}^{1} q_{2}(x) d x},
$$

usando el hecho de que $A=1 /(1+\alpha)$ separa al intervalo $(0,1)$ en dos partes cuya proporción es $\alpha$. Entonces, luego de generar un valor para $R$, si $R>\alpha$ se muestrea de $q_{1}$, y si $R<\alpha$ se muestrea de $q_{2}$.

La mayoría de las reglas de muestreo que se han implementado en el código para simulación de cascadas desarrollado, siguen o bien estas simples líneas, o las recetas específicas dadas en Protheroe (1986).

\section{A.5. Las ecuaciones que describen una cascadas electro- magnética}

El planteo de una ecuación de difusión como en la expresión (8.8) para describir la evolución de una dada población de electrones, es válido en tanto que los procesos que hacen perder energía a los electrones sean en cierto sentido "suaves". La energía perdida (ya sea por radiación o colisión) en una interacción de un dado electrón debe ser mucho menor que su propia energía. Esta condición se expresa

$$
-\dot{E} / E \ll N c \sigma
$$

siendo $\dot{E}$ la tasa de pérdida de energía, $\sigma$ la sección eficaz total, y $N$ la densidad numérica en el medio (Blumenthal \& Gould 1970). En ciertos regimenes, procesos radiativos como 
el Bremsstrahlung y el Compton inverso (en el límite de Klein-Nishina) no cumplen con (A.37), ya que el electrón transfiere una fracción importante de su energía a un sólo fotón. Entonces se hace necesario considerar un planteo diferente, que permite contemplar el desarrollo de cascadas electromagnéticas en un medio.

Considerese una situación longitudinal, donde $t$ es el parámetro de la profundidad, y el medio puede tener una estructura arbitraria. Llamaremos $f(t, E) d E$ al número medio de electrones por unidad de volumen con energía entre $E$ y $E+d E$ a la profundidad $t$. En forma análoga denotamos por $g(t, E) d E$ al número medio de fotones (espectro diferencial). Las ecuaciones de propagación se deducen considerando los procesos de absorción y emisión que tienen lugar en una capa de espesor diferencial $d t$. Este planteo analítico fue desarrollado en principio por Carlson y Oppenheimer, y perfeccionado por Landau \& Rumer (1938) entre otros. El conjunto de ecuaciones acopladas que resultan tiene esta forma

$$
\begin{aligned}
& \lambda_{e} \frac{\partial f}{\partial t}+f-\int_{E}^{\infty} w_{e e}\left(E, E^{\prime}\right) f\left(t, E^{\prime}\right) d E^{\prime}-\int_{E}^{\infty} w_{e \gamma}\left(E, E^{\prime}\right) g\left(t, E^{\prime}\right) d E^{\prime}=\lambda_{e} F, \\
& \lambda_{\gamma} \frac{\partial g}{\partial t}+g-\int_{E}^{\infty} w_{\gamma e}\left(E, E^{\prime}\right) f\left(t, E^{\prime}\right) d E^{\prime}-\int_{E}^{\infty} w_{\gamma \gamma}\left(E, E^{\prime}\right) g\left(t, E^{\prime}\right) d E^{\prime}=\lambda_{\gamma} G,
\end{aligned}
$$

donde $\lambda_{\alpha}$ es el camino libre medio para una partícula de tipo $\alpha(\alpha=e$ o $\gamma)$ y puede expresarse a través de la sección eficaz total. A través de $F$ y $G$ se introducen las condiciones de contorno. Las funciones $w_{\alpha \beta}\left(E, E^{\prime}\right)$ definen el espectro diferencial (sobre la energía $E^{\prime}$ ) de partículas secundarias de tipo $\beta(\beta=e$ o $\gamma)$. Notese que estas funciones aparecen convolucionadas con los espectros diferenciales de modo que el resultado de los términos integrados es el número de partículas de tipo $\alpha$ con energía $E$ que se obtienen como secundarias en la interacción de partículas $\beta$, de energía $E^{\prime}$ necesariamente mayor. El acoplamiento de estas ecuaciones integro-diferenciales es explícito y puede resumirse bajo el concepto: por interacción con el medio, los fotones materializan pares y los electrones emiten fotones.

Aharonian \& Plyasheshnikov (2003) brindan una descripción detallada de un sistema de ecuaciones similar al transcripto arriba, junto con la descripción de un método numérico para la resolución del mismo, en casos de medios homogéneos. En Rossi (1952) puede hallarse una descripción más accesible, para el caso de la aplicación a un medio material bajo la llamada Aproximación A. 


\section{Lista de publicaciones}

\section{En revistas internacionales:}

- Gamma-Ray Emission From Be/X-Ray Binaries

M. Orellana \& G.E. Romero

Astrophysics and Space Science, 2005, 297, 167 - 178. [astro-ph/0407450]

- Gamma-ray and neutrino emission from misaligned microquasars

G. E. Romero \& M. Orellana

Astronomy \& Astrophysics, 2005, 439, 237 - 244. [astro-ph/0505287]

- Hadronic high-energy gamma-ray emission from the microquasar LS I +61303

G. E. Romero, H. R. Christiansen, M. Orellana

Astrophysical Journal, 2005, 632, 1093 - 1098. [astro-ph/0506735]

- High-energy gamma-ray emission from the inner jet of LS I+61 303: the hadronic contribution revisited

M. Orellana \& G. E. Romero

Astrophysics and Space Science, 2007, 309, 333-338. [astro-ph/0608707]

- Synchrotron emission from secondary leptons in microquasar jets

P. Bordas, J.M. Paredes, V. Bosch-Ramon, M. Orellana

Astrophysics and Space Science, 2007, 309, 339-343. [astro-ph/0710.0545]

- Very high energy $\gamma$-ray emission from X-ray transients during major outbursts

M. Orellana, G. E. Romero, L. J. Pellizza, S. Vidrih

Astronomy \& Astrophysics, 2007,465, 703-709. [astro-ph/0701695] 
- Accretion vs colliding wind models for the gamma-ray binary LS I +61 303: an assessment

G.E. Romero, A.T. Okazaki, M. Orellana, S.P. Owocki

Astronomy \& Astrophysics, 2007, 474, 15-22. [astro-ph/0706.1320]

- Leptonic secondary emission in a hadronic microquasar model

M. Orellana, P. Bordas, V. Bosch-Ramon, G. E. Romero, J. M. Paredes

Astronomy \& Astrophysics, 2007, 476, 9-15. [astro-ph/0710.0751]

\section{Publicaciones en actas de congresos:}

- On the nature of the unidentified MeV gamma-ray source GRO J1411-64 G.E. Romero, M. Orellana, D. F. Torres ESA-SP, 2004, 552, 707 - 710. [astro-ph/0403333]

- Hadronic gamma-ray production in microquasars with equatorial winds M. Orellana \& G. E. Romero RevMexAA(SC), 2006, 26, 145 - 146.

- Gamma-ray emission from microquasars: leptonic vs hadronic models G.E. Romero, V. Bosch-Ramon, J. M. Paredes, M. Orellana. Springer-Verlag: ESO Astrophysics Symposia, en prensa (2005)

- Electromagnetic cascades in the radiation field of massive stars M. Orellana \& G.E. Romero Enviado, para aparecer en los Proceedings del workshop "Massive stars: Fundamental Parameters and Circumstellar Interactions", RevMexAA(SC), 2007

- High-energy emission from jet-wind and jet-star interactions in misaligned microquasars

M. Orellana \& G. E. Romero

Boletín de la Asociación Argentina de Astronomía, Vol. 47, pag 291-295.

- High-energy emission from accreting Be/X-ray binary systems G.E. Romero \& M. Orellana Boletín de la Asociación Argentina de Astronomía, Vol. 47, pag 296-299.

- Emisión de origen hadrónico en microquasares con vientos M. Orellana \& G.E. Romero.

Boletín de la Asociación Argentina de Astronomía, Vol. 48, pag 334-340. 
- Electromagnetic cascades in early-type binary systems

M. Orellana, G.E. Romero, L.J. Pellizza

Boletín de la Asociación Argentina de Astronomía, Vol 49, 330-333 (2006).

- Gamma-ray emission from the microquasar LSI+61 303

G.E. Romero \& M. Orellana

Boletín de la Asociación Argentina de Astronomía, 2006, Vol. 49, 338-341.

- Gamma-ray emission from microquasars: leptonic vs. hadronic models

G.E. Romero, V. Bosch-Ramon, J.M. Paredes, M. Orellana.

B. Aschenbach, V. Burwitz, G. Hasinger, \& B. Leibundgut (eds), Relativistic astrophysics and cosmology -Einstein's legacy, Springer, Berlin-Heidelberg, pp. 480-482, 2007.

- LS I +61 303: microquasar or not microquasar?

G.E. Romero, M. Orellana, A.T. Okazaki, S.P. Owocki

Proceedings del workshop "High Energy Processes in Relativistic Outflows", Dublin, Septiembre, 2007, en prensa [astro-ph/0712.1832] 
